

\section{Switchable Adhesion and Friction by Stimulus Responsive Polymer Brushes}

\section{Yunlong Yu}




\section{Graduation committee}

\section{Chairman}

Prof. dr. ir. J.W.M. Hilgenkamp

University of Twente

\section{Promotor}

Prof. dr. G. J. Vancso

University of Twente

\section{Assistant-promotor}

Dr. S.J.A. de Beer

University of Twente

\section{Members}

Prof. dr. X. Zeng

Shanghai Advanced Research Institute, Chinese Academy of Sciences

Dr. A.C.C. de Carvalho Esteves

Eindhoven University of Technology

Prof. dr. M.M.A.E. Claessens

University of Twente

Prof. dr. J.J.L.M. Cornelissen

University of Twente

Dr. W.M. de Vos

University of Twente

The research described in this Thesis was carried out in the Materials Science and Technology of Polymers (MTP) group, in the MESA+ Institute for Nanotechnology, and in the Faculty of Science and Technology (TNW) of the University of Twente. This research was supported by the China Scholarship Council (CSC) and by the Netherlands Organization for Scientific Research (NWO).

\section{Title: Switchable Adhesion and Friction by Stimulus Responsive Polymer Brushes}

Copyright (C) 2017, Yunlong Yu, Enschede, the Netherlands

All rights reserved. No part of this Thesis may be reproduced or transmitted by print, photocopy or any other means without prior written permission of the author.

ISBN: 978-90-365-4280-7

DOI: $10.3990 / 1.9789036542807$

Cover: designed by GR-Artworks-Geneviève Rietveld

Printed by Ipskamp Drukkers in Enschede, the Netherlands 


\title{
SWITCHABLE ADHESION AND FRICTION BY STIMULUS RESPONSIVE POLYMER BRUSHES
}

\author{
DISSERTATION \\ to obtain \\ the degree of doctor at the University of Twente, \\ on the authority of the rector magnificus, \\ prof. dr. T.T.M. Palstra,
} on account of the decision of the graduation committee, to be publicly defended on Wednesday, $11^{\text {th }}$ of January 2017 , at $14: 45 \mathrm{~h}$

Yunlong Yu

born on $26^{\text {th }}$ of October, 1984

in Shandong, P. R. China 
This dissertation has been approved by:

Promotor: Prof. dr. G. J. Vancso

Assistant-promotor: Dr. S.J.A. de Beer 


\section{Contents}

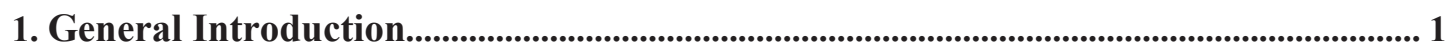

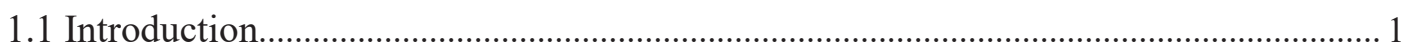

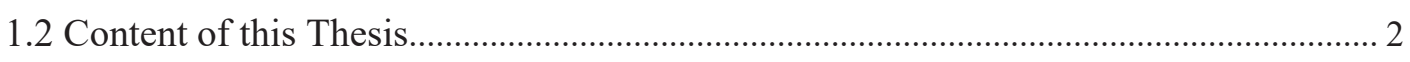

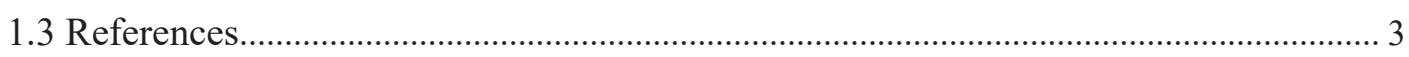

2. Switchable Adhesion and Friction by Stimulus Responsive Polymers........................... 5

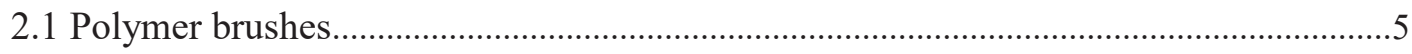

2.2 Surface-initiated atom transfer radical polymerization (SI-ATRP) ................................

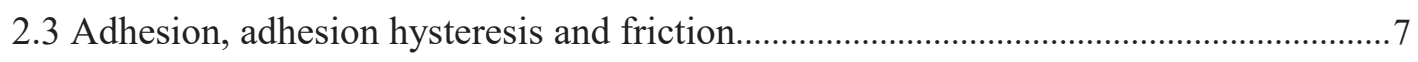

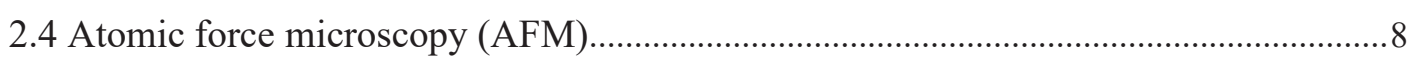

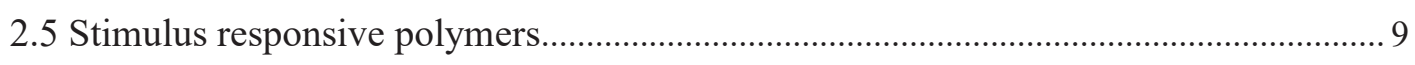

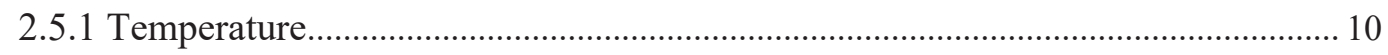

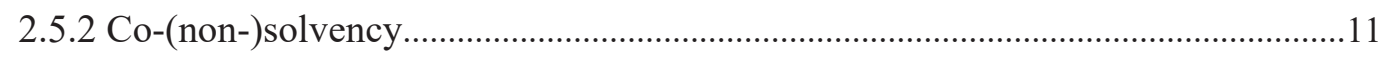

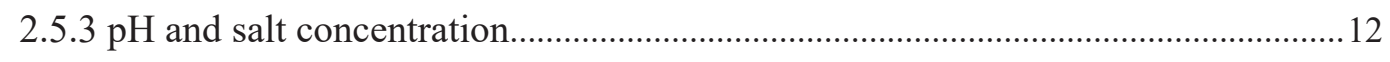

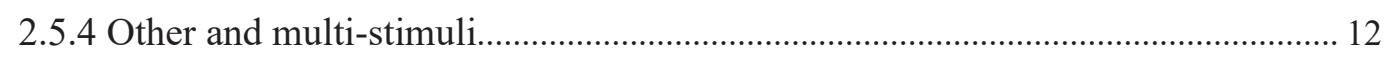

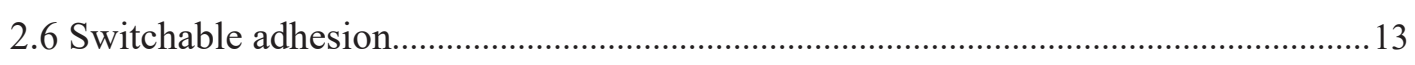

2.6.1 Adhesion between chemically identical polymer films......................................... 13

2.6.2 The adhesion between polymer films and chemically different counter surfaces 15

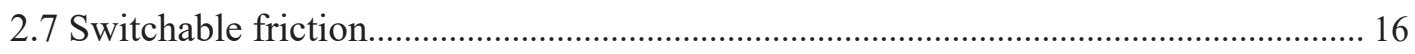

2.7.1 Friction between chemically identical polymer films.......................................... 17

2.7.2 Friction between polymer films and chemically different counter surfaces......... 17

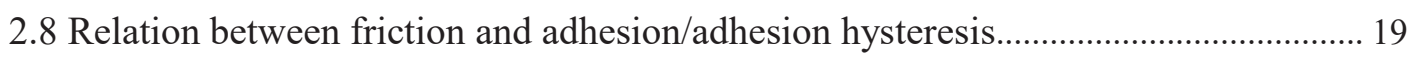

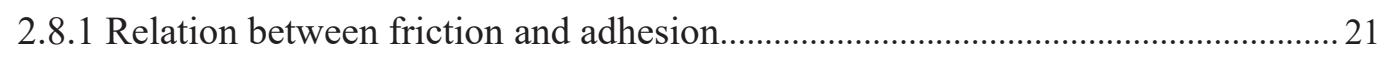

2.8.2 Relation between friction and adhesion hysteresis................................................2

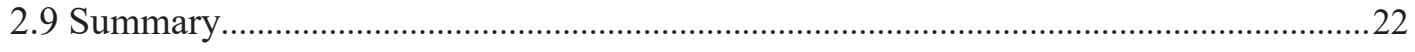

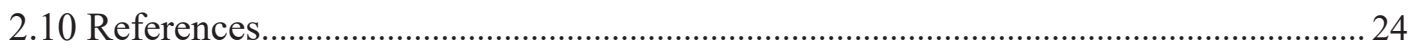

\section{Stretching of Collapsed Polymers Causes an Enhanced Dissipative Response of} PNIPAM Brushes near their LCST..............................................................................31

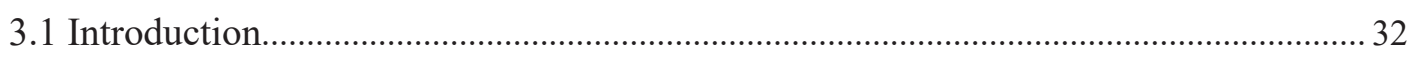

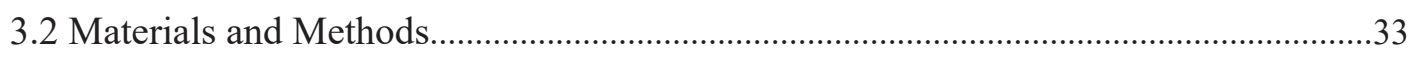

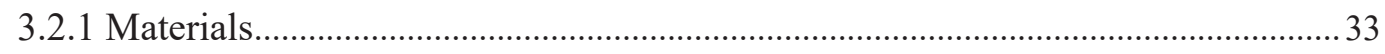

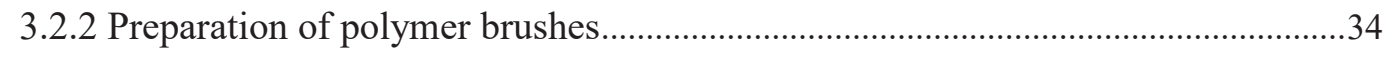

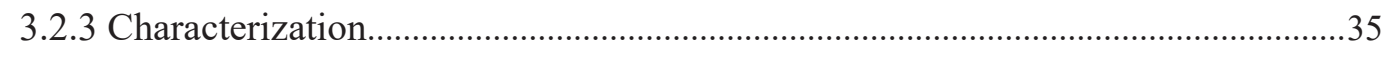

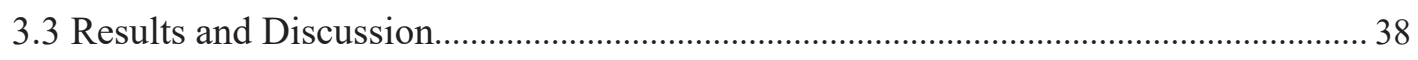

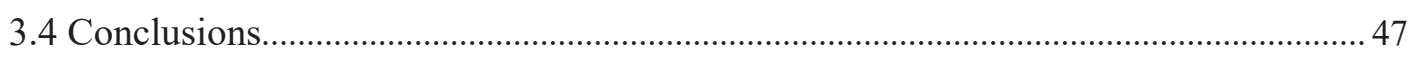




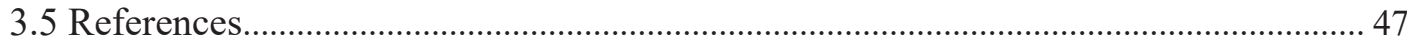

4. Tunable Friction by Employment of Co-non-solvency of PNIPAM Brushes..............51

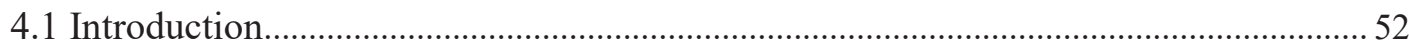

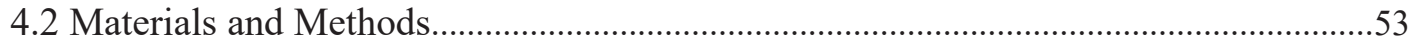

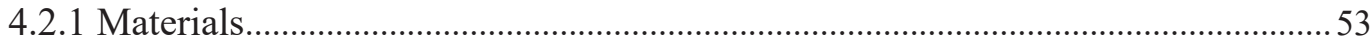

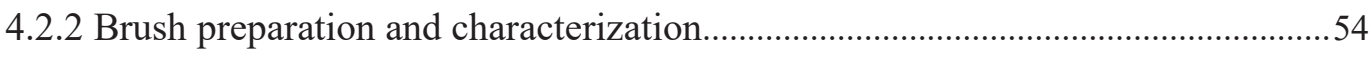

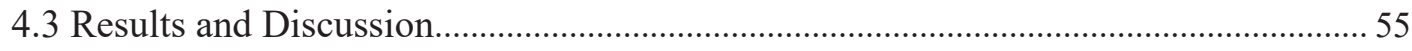

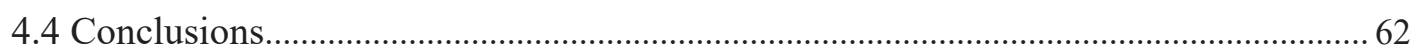

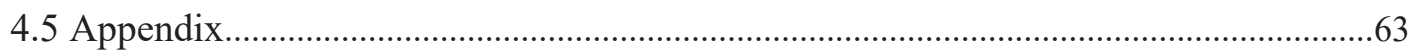

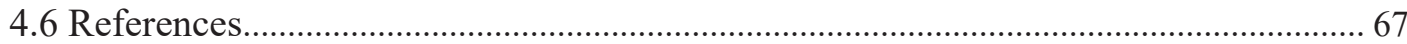

5. Pick up, Move and Release of Nanoparticles Utilizing Co-non-solvency of PNIPAM

Brushes............................................................................................................................................................. 71

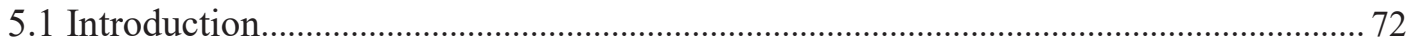

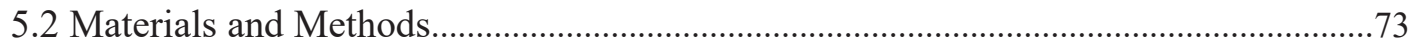

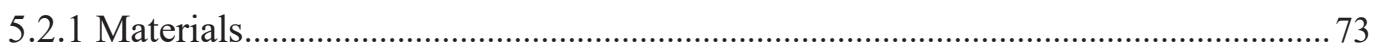

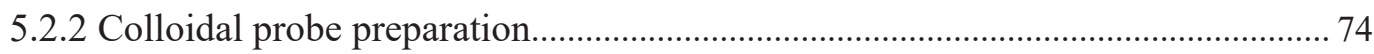

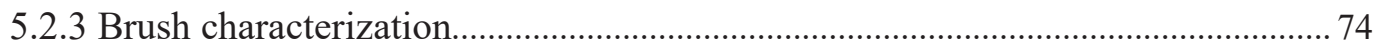

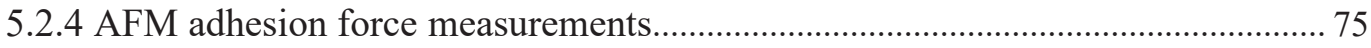

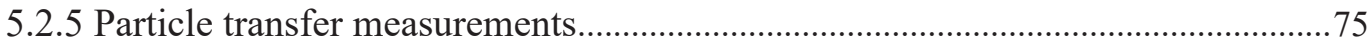

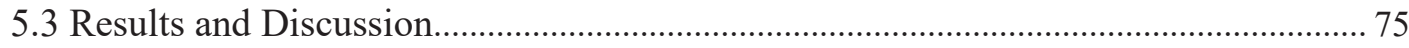

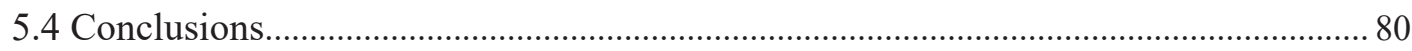

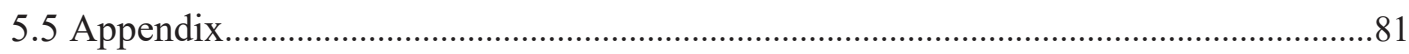

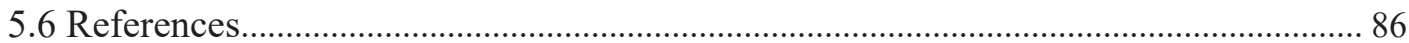

6. Cosolvency-Induced Switching of the Adhesion between Poly(methyl methacrylate)

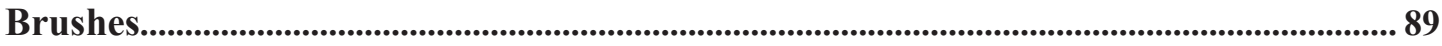

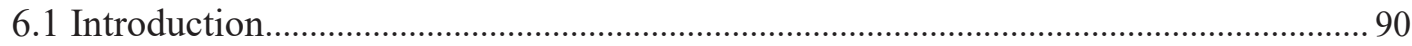

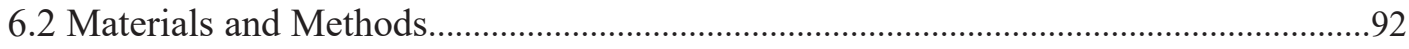

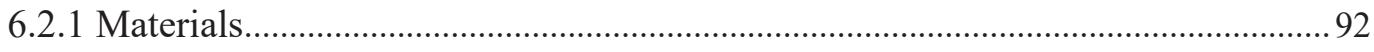

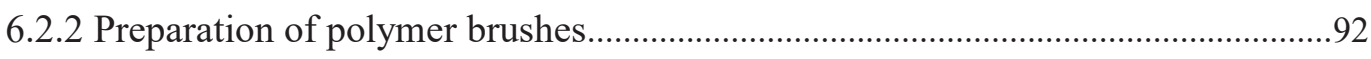

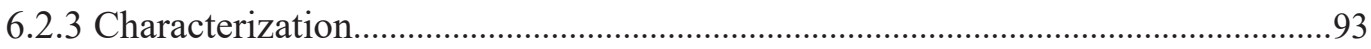

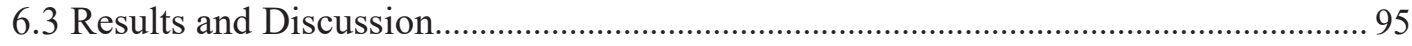

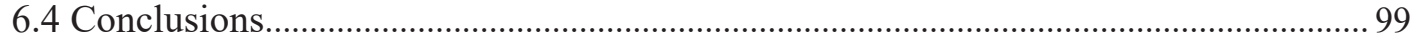

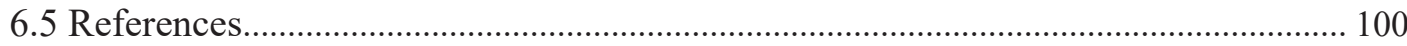

7. Substantially Enhanced Stability Against Degrafting of Zwitterionic Brushes by Utilizing PGMA-based Macro-initiators......................................................................................... 103

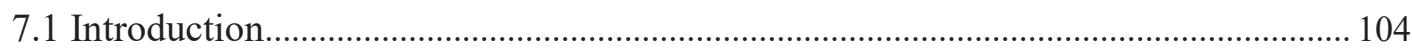

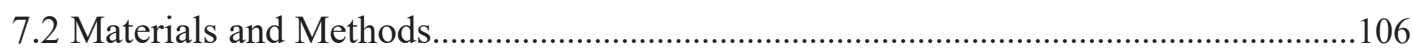




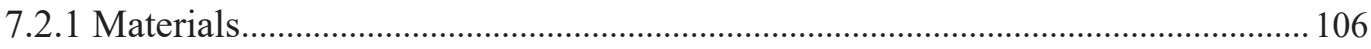

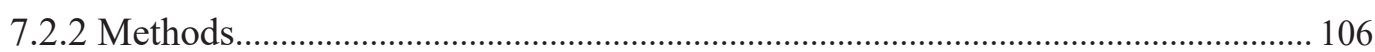

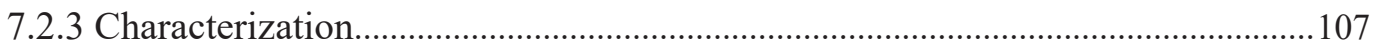

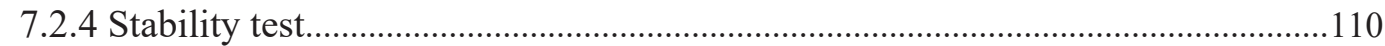

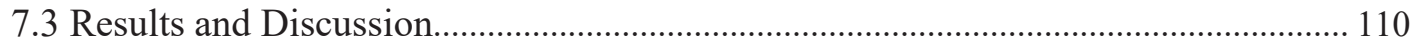

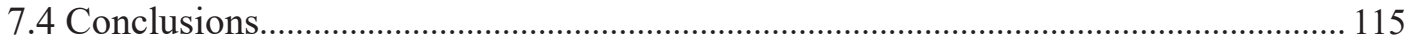

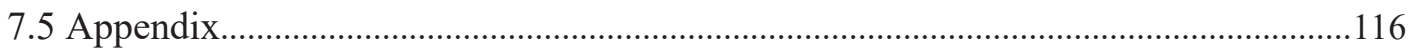

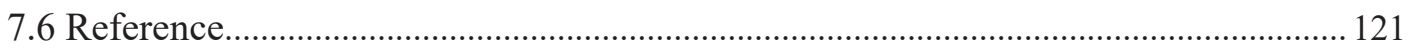

8. First Results and Outlook on: Specific Anion Effects on the Hydration and Tribological properties of Zwitterionic PMPC Brushes..........................................................125

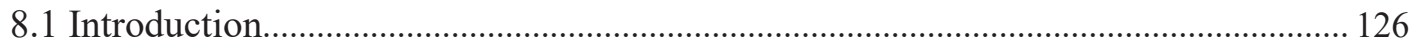

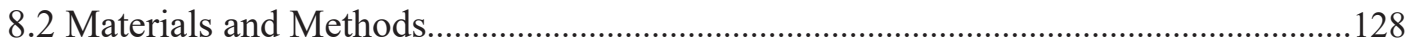

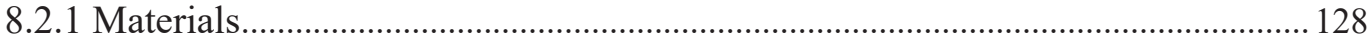

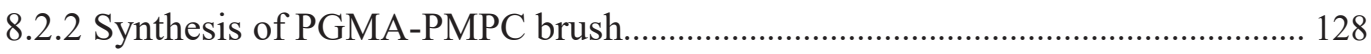

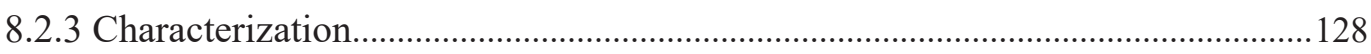

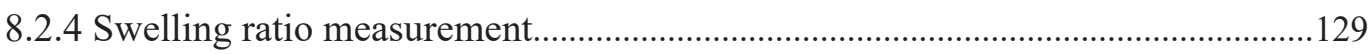

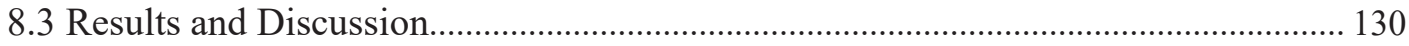

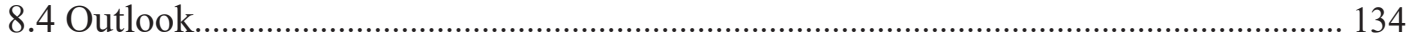

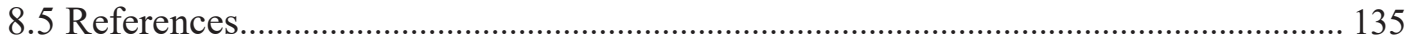

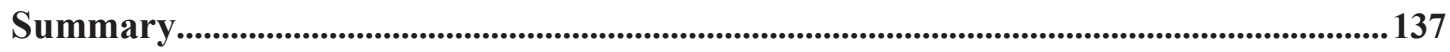

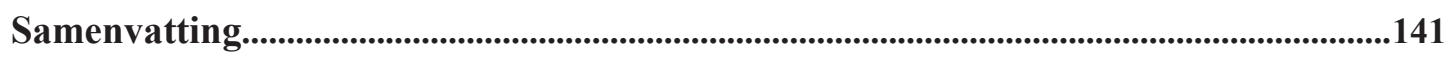

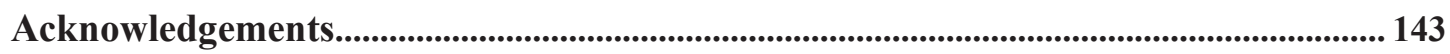

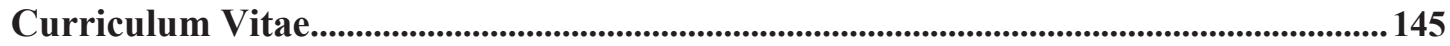





\section{Chapter 1}

\section{General Introduction}

\subsection{Introduction}

Stimulus-responsive polymers ${ }^{1}$ are defined as "polymers that undergo relatively large and abrupt, physical or chemical changes in response to small modifications in the environmental conditions". Recently, there has been quickly growing interest in developing 'smart' surface-coatings by using stimulus-responsive polymers, while they can be applied for varying the surface wettability ${ }^{2}$ and in drug delivery, ${ }^{3}$ chemical-sensing ${ }^{4}$ and tribology control. ${ }^{5}$ In these applications, the conformational state and/or the chemical structure of the polymers is strongly altered due to the adjustment in the surroundings, such as temperature ${ }^{6}$ light radiation ${ }^{7}$ or electric field, ${ }^{8}$ which results in a change in the macroscopic properties of the surface coating.

There are different methods to decorate a surface with polymers. Polymers can be physisorbed $^{9}$ or covalently bonded to the surface via their side-chains. ${ }^{10}$ Moreover, hydrogels can be prepared, which can swell by solvent absorption by many times their initial size. ${ }^{11}$ Furthermore, so-called polymer brushes can be formed. ${ }^{12}$ In a polymer brush, the polymers are attached with one end to the surface at a density that is high enough for the polymers to stretch away from the surface. ${ }^{13}$ The latter is caused by steric hindrance by the surrounding polymers and can be enhanced by solvent absorption when the brush is kept in a good solvent.

In this Thesis, we will present different polymer brush coatings that have been used to control adhesion and friction. When polymers are triggered to change their conformation or chemical structure by an external stimulus, the interaction, e.g. the van der Waals forces, electrostatic forces, forces due to hydrogen bonding etc., between surfaces that are coated with these polymers will also change. Generally, when a stimulus causes the effective interaction to become more attractive, the surfaces will be more strongly bound and make more intimate contact, such that adhesion and friction will increase, while the opposite is true when the stimulus results in more repulsive interactions. ${ }^{5 b, 6 b, 14}$ However, as we will discuss in more detail in this Thesis, there are important exceptions for which the inverse can occur. ${ }^{12 b,}{ }^{15}$ In our research we systematically study the relation between the degree of solvation of the macromolecules in the brush and the friction and adhesion. Our results show that the relation between brush swelling and its tribomechanical properties is rather complex, and that it can depend on the specific interactions in the contact. Our careful characterization will allow for the development of e.g. smart tweezers ${ }^{5 \mathrm{e}}$ and gloves, ${ }^{16}$ pick up and release tools. ${ }^{17}$ 
Besides coating surfaces with polymers, there are alternative methods to tune adhesion and friction, which we will not be the focus of this Thesis. For example, stick-slip motion can be suppressed using mechanical oscillations of macroscopic surfaces ${ }^{18}$ or atomically flat surfaces. ${ }^{19}$ Between elastic membranes and rigid counter faces, friction can be altered by wrinkles. ${ }^{20}$ Moreover, friction between atomically flat metal surfaces and an atomic force microscopy tip can be controlled using surface oxidation ${ }^{21}$ or surface reconstruction. ${ }^{22}$ For example, the friction on atomically flat $\mathrm{Au}$ (111) surfaces can be switched from high to low reversibly by oxidation and reduction. ${ }^{21}$ Also Vezenov et al. ${ }^{23}$ modified surface of Si (100) or gold with $-\mathrm{NH}_{2}$ or $-\mathrm{COOH}$ group. When varying $\mathrm{pH}$, both adhesion and friction can be tuned. All these methods require ideal surfaces and/or low-contamination-levels. Stimulus responsive polymer coatings are relatively cheap and robust alternative to these methods.

\subsection{Content of this Thesis}

In this Thesis, we present methods to fabricate smart surfaces using stimulus-responsive polymers, with which we achieve switchable adhesion and friction. The main theme of each chapter is as following.

Chapter 2 provides a background of the techniques employed in this Thesis and summarizes the research reported on switchable friction and adhesion using stimulusresponsive polymer films, gels and brushes so far.

Chapter 3 reports on switchable friction and adhesion using thermally responsive PNIPAM brushes. An enhanced dissipation and friction are observed near the lower critical solution temperature (LCST) of PNIPAM, which we attribute to stretching of partly collapsed polymer brush chains that adhere to the gold colloid probe used in assessing the interfacial properties.

Chapter 4 explores the enhanced friction and dissipation of PNIPAM brushes due to the co-non-solvency effect. Both in water and in ethanol, low friction is obtained due to the high osmotic pressure of good solvents in the brush. However, in 10\% volume fraction of ethanol-water composition, a maximum in friction is observed. The highest friction is about two orders of magnitude larger than the lowest friction.

Chapter 5 focuses on the application of co-non-solvency of PNIPAM brushes to pick up, move and release nanoparticles. In a water-ethanol mixture of $30 \%$ ethanol, the brushparticle adhesion is high such that particles can be picked up. In pure solvent, the brushes swell and the adhesion is strongly reduced such that particle-release can be triggered.

Chapter 6 describes switchable adhesion between PMMA brushes utilizing the cosolvency effect. Water, ethanol and isopropanol are poor solvents for PMMA and we observe a high contact adhesion between PMMA brushes in these pure solvents. However, in a mixture of water and one of the alcohols $(\mathrm{v} / \mathrm{v}=4 / 5)$ the brushes swell resulting in a low adhesion. 
Chapter 7 introduces a new method to fabricate zwitterionic PMPC brushes using a PGMA-based macro-initiator. We show that degrafting is effectively inhibited in aqueous media, physiological media (saline), and marine environment ( $0.6 \mathrm{M}$ sodium chloride).

Chapter 8 investigates the specific ion effect on the hydration of zwitterionic PMPC brushes to control friction and adhesion. Preliminary results show that salts have an effect on the swelling ratio of PMPC brushes and on their friction and adhesion with a rigid counter-surface. An outlook to future experiments is going to help us to clarify our observations.

\subsection{References}

[1] a) M. A. C. Stuart, W. T. S. Huck, J. Genzer, M. Muller, C. Ober, M. Stamm, G. B. Sukhorukov, I. Szleifer, V. V. Tsukruk, M. Urban, F. Winnik, S. Zauscher, I. Luzinov and S. Minko, Nature Materials 2010, 9, 101-113; b) E. S. Gil and S. M. Hudson, Progress in Polymer Science 2004, 29, 1173-1222; c) F. Liu and M. W. Urban, Progress in Polymer Science 2010, 35, 3-23.

[2] a) A. M. Jonas, Z. J. Hu, K. Glinel and W. T. S. Huck, Macromolecules 2008, 41, 6859-6863; b) L. Feng, S. H. Li, Y. S. Li, H. J. Li, L. J. Zhang, J. Zhai, Y. L. Song, B. Q. Liu, L. Jiang and D. B. Zhu, Advanced Materials 2002, 14, 1857-1860; c) A. C. de Leon and R. Advincula, Abstracts of Papers of the American Chemical Society 2013, 246; d) T. L. Sun, G. J. Wang, L. Feng, B. Q. Liu, Y. M. Ma, L. Jiang and D. B. Zhu, Angewandte Chemie-International Edition 2004, 43, 357-360.

[3] a) Q. Yuan, R. Venkatasubramanian, S. Hein and R. D. K. Misra, Acta Biomaterialia 2008, 4, 1024-1037; b) H. Y. Tian, Z. H. Tang, X. L. Zhuang, X. S. Chen and X. B. Jing, Progress in Polymer Science 2012, 37, 237-280.

[4] a) O. Azzaroni, B. Trappmann, P. van Rijn, F. Zhou, B. Kong and W. T. S. Huck, Angewandte ChemieInternational Edition 2006, 45, 7440-7443; b) N. I. Abu-Lail, M. Kaholek, B. LaMattina, R. L. Clark and S. Zauscher, Sensors and Actuators B-Chemical 2006, 114, 371-378; c) I. Tokareva, S. Minko, J. H. Fendler and E. Hutter, Journal of the American Chemical Society 2004, 126, 15950-15951.

[5] a) D. P. Chang, J. E. Dolbow and S. Zauscher, Langmuir 2007, 23, 250-257; b) S. de Beer, Langmuir 2014, 30, 8085-8090; c) S. H. Ma, D. A. Wang, Y. M. Liang, B. Q. Sun, S. N. Gorb and F. Zhou, Small 2015, 11, 1131-1137; d) Q. B. Wei, M. R. Cai, F. Zhou and W. M. Liu, Macromolecules 2013, 46, 9368-9379; e) Y. Wu, M. R. Cai, X. W. Pei, Y. M. Liang and F. Zhou, Macromolecular Rapid Communications 2013, 34, 1785-1790; f) Y. Wu, Q. B. Wei, M. R. Cai and F. Zhou, Advanced Materials Interfaces 2015, 2.

[6] a) E. Bittrich, S. Burkert, M. Muller, K. J. Eichhorn, M. Stamm and P. Uhlmann, Langmuir 2012, 28, 3439-3448; b) S. Desseaux and H. A. Klok, Biomacromolecules 2014, 15, 3859-3865; c) O. Tagit, N. Tomczak, E. M. Benetti, Y. Cesa, C. Blum, V. Subramaniam, J. L. Herek and G. J. Vancso, Nanotechnology 2009, 20; d) H. Yim, M. Kent, S. Mendez, G. Lopez, S. Satija and Y. Seo, Macromolecules 2006, 39, 34203426.

[7] a) F. Ercole, T. P. Davis and R. A. Evans, Polymer Chemistry 2010, 1, 37-54; b) O. Y. Jin, D. W. Fu, J. Wei, H. Yang and J. B. Guo, Rsc Advances 2014, 4, 28597-28600; c) W. X. Hou and Q. H. Wang, Langmuir 2009, 25, 6875-6879; d) H. S. Lim, D. Kwak, D. Y. Lee, S. G. Lee and K. Cho, Journal of the American Chemical Society 2007, 129, 4128-4129.

[8] a) T. Tanaka, I. Nishio, S. T. Sun and S. Uenonishio, Science 1982, 218, 467-469; b) N. Fujiwara, K. Asaka, Y. Nishimura, K. Oguro and E. Torikai, Chemistry of Materials 2000, 12, 1750-1754; c) H. M. Zhang, J. J. Li, H. T. Cui, H. J. Li and F. L. Yang, Chemical Engineering Journal 2015, 259, 814-819.

[9] a) A. Dedinaite, E. Thormann, G. Olanya, P. M. Claesson, B. Nystrom, A. L. Kjoniksen and K. Z. Zhu, Soft Matter 2010, 6, 2489-2498; b) S. Kessel, S. Schmidt, R. Muller, E. Wischerhoff, A. Laschewsky, J. F. Lutz, K. Uhlig, A. Lankenau, C. Duschl and A. Fery, Langmuir 2010, 26, 3462-3467; c) X. Y. Liu, A. Dedinaite, M. Rutland, E. Thormann, C. Visnevskij, R. Makuska and P. M. Claesson, Langmuir 2012, 28, 15537-15547.

[10] a) T. Chen, R. Ferris, J. M. Zhang, R. Ducker and S. Zauscher, Progress in Polymer Science 2010, 35 , 94-112; b) S. Edmondson, V. L. Osborne and W. T. S. Huck, Chemical Society Reviews 2004, 33, $14-22$.

[11] H. K. Ju, S. Y. Kim and Y. M. Lee, Polymer 2001, 42, 6851-6857. 
[12] a) S. B. Rahane, J. A. Floyd, A. T. Metters and S. M. Kilbey, Advanced Functional Materials 2008, 18, 1232-1240; b) Y. L. Yu, B. D. Kieviet, F. Liu, I. Siretanu, E. Kutnyanszky, G. J. Vancso and S. de Beer, Soft Matter 2015, 11, 8508-8516.

[13] S. T. Milner, Science 1991, 251, 905-914.

[14] a) S. Choi, B. C. Choi, C. Y. Xue and D. Leckband, Biomacromolecules 2013, 14, 92-100; b) G. Q. Liu, Z. L. Liu, N. Li, X. L. Wang, F. Zhou and W. M. Liu, Acs Applied Materials \& Interfaces 2014, 6, $20452-$ 20463; c) C. Rodriguez-Emmenegger, C. M. Preuss, B. Yameen, O. Pop-Georgievski, M. Bachmann, J. O. Mueller, M. Bruns, A. S. Goldmann, M. Bastmeyer and C. Barner-Kowollik, Advanced Materials 2013, 25, 6123-6127; d) E. Svetushkina, N. Puretskiy, L. Ionov, M. Stamm and A. Synytska, Soft Matter 2011, 7, 56915696; e) J. T. Yang, H. Chen, S. W. Xiao, M. X. Shen, F. Chen, P. Fan, M. Q. Zhong and J. Zheng, Langmuir 2015, 31, 9125-9133; f) R. Zhang, S. H. Ma, Q. B. Wei, Q. Ye, B. Yu, J. van der Gucht and F. Zhou, Macromolecules 2015, 48, 6186-6196; g) Z. Y. Zhang, A. J. Morse, S. P. Armes, A. L. Lewis, M. Geoghegan and G. J. Leggett, Langmuir 2011, 27, 2514-2521; h) Z. Y. Zhang, A. J. Morse, S. P. Armes, A. L. Lewis, M. Geoghegan and G. J. Leggett, Langmuir 2013, 29, 10684-10692.

[15] J. Huang, B. Cusick, J. Pietrasik, L. Wang, T. Kowalewski, Q. Lin and K. Matyjaszewski, Langmuir 2007, 23, 241-249.

[16] S. Ma, H. Lee, Y. Liang and F. Zhou, Angewandte Chemie International Edition 2016.

[17] Y. L. Yu, B. D. Kieviet, E. Kutnyanszky, G. J. Vancso and S. de Beer, Acs Macro Letters 2015, 4, 75-79.

[18] L. Bureau, T. Baumberger and C. Caroli, Physical Review E 2000, 62, 6810-6820.

[19] A. Socoliuc, E. Gnecco, S. Maier, O. Pfeiffer, A. Baratoff, R. Bennewitz and E. Meyer, Science 2006, $313,207-210$.

[20] H. Mohammadi and M. H. Muser, Physical Review Letters 2010, 105.

[21] A. Labuda, F. Hausen, N. N. Gosvami, P. H. Grutter, R. B. Lennox and R. Bennewitz, Langmuir 2011, $27,2561-2566$.

[22] F. Hausen, J. A. Zimmet and R. Bennewitz, Surface Science 2013, 607, 20-24.

[23] D. V. Vezenov, A. Noy, L. F. Rozsnyai and C. M. Lieber, Journal of the American Chemical Society 1997, 119, 2006-2015. 


\section{Chapter 2}

\section{Switchable Adhesion and Friction by Stimulus Responsive Polymers}

In this chapter, we present a general background to the topics and techniques discussed and employed in this Thesis. Moreover, we will give a literature overview of the present state of the research on switchable adhesion and friction using surface-attached stimulus responsive polymers.

\subsection{Polymer brushes}

Polymer brushes consist of macromolecules that are constrained with one end at a surface or an interface at a density that is sufficiently high such that the polymers stretch away from the grafting plane. ${ }^{1}$ Not all tethered polymers can form brushes. Depending on the grafting density and the molecular weight of polymers on the substrates, there can be various conformations of the polymer(-film), e.g. mushrooms or pancakes (depending on the surface-polymer-solvent interaction), mushroom-to-brush transitions and brushes (shown in Figure 2.1). When the distance between anchor points $a$ is more than twice as large as the radius of gyration of polymers ( $a>2 R_{\mathrm{gyr}}$ ), there is almost no interaction between two single chains. ${ }^{2}$ This distance can be translated to the critical grafting density, defined as $\sigma^{*}=$ $1 /\left(\pi^{*} R_{\mathrm{gyr}}{ }^{2}\right),{ }^{2 \mathrm{c}, 2 \mathrm{~d}}$ which can be employed to identify the mushroom-to-brush transition in the absence of attractive interactions with the wall: When $\sigma<\sigma^{*}$, a mushrooms are formed. When $\sigma^{*}<\sigma<5 \sigma^{*}$, a transition from mushroom to brush occurs. If $\sigma>5 \sigma^{*}$, the brushes are formed. $^{2 \mathrm{a}, 3}$ In the presence of interactions with the wall, there can be pancakes (strong attractive interaction) or mushroom (weak or repulsive interaction) states for $\sigma<\sigma^{*}$, depending on the strength of interaction between surface-polymer $\left(\varepsilon_{\mathrm{wp}}\right)$ and surface-solvent $\left(\varepsilon_{\mathrm{ws}}\right) .{ }^{4}$ To be more specific, in a dilute polymer solution, the solubility of polymers can be determined by a single dimensionless parameter $\chi$, which expresses the strength of the energetic interaction between polymer and solvent. ${ }^{5}$ When $\chi>0.5$, the polymer chains collapses to form a globule. While, when $\chi<0.5$, the polymer chain is expanded to swell in the solvent. For $\chi>0.5$ and when $\varepsilon_{\mathrm{wp}}>\varepsilon_{\mathrm{ws}}$, the polymer chain sticks to the substrate and forms a pancake. For $\chi<0.5$ and when $\varepsilon_{\mathrm{ws}}>\varepsilon_{\mathrm{wp}}$, the polymer will extend to form a mushroom state. With a decrease of the distance between two anchor points to less than twice of the radius of gyration $\left(\sigma^{*}<\sigma<5 \sigma^{*}\right)$, the polymer chain-chain interaction starts to increase, and a mushroom to brush transition happens. For short polymers at high grafting densities $\left(\sigma>5 \sigma^{*}\right)$, there are two possible states. In a poor solvent, or if the surface-polymer interaction dominates $\left(\varepsilon_{\mathrm{wp}}>\varepsilon_{\mathrm{ws}}\right)$, a dense polymer film is formed on the substrate. While in 
a good solvent and if the surface-solvent interactions dominate $\left(\varepsilon_{\mathrm{ws}}>\varepsilon_{\mathrm{wp}}\right)$, polymer brushes can be obtained where the polymer chains are absorbing the solvent. With long polymer chains and in a good solvent, normally stretched polymer brushes are formed. ${ }^{6}$ Even if there is a high $\varepsilon_{\mathrm{wp}}$, the brush can swell and stretch perpendicular to the substrate plane and there is only a dense film layer near the substrate. In a poor solvent and when $\varepsilon_{\mathrm{wp}}$ dominates; a dense film layer is found. ${ }^{7}$

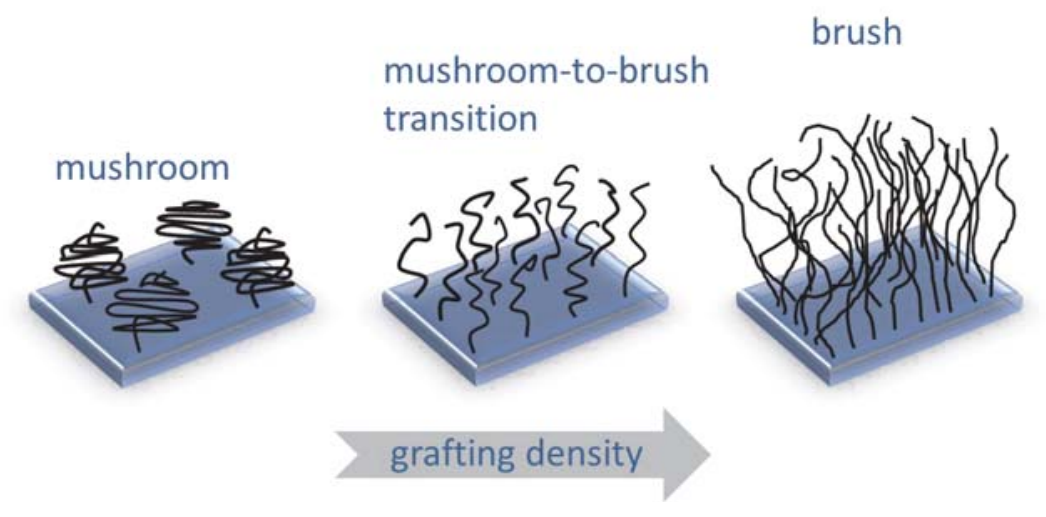

Figure 2.1. Scheme of the conformations of tethered polymer chains on the surface with different grafting densities: mushroom, mushroom-to-brush transition, brush.

There are two methods to attach polymer brushes on the substrate: "grafting to" 8 and "grafting from". 9 In general, "grafting to" leads a loose attachment and a low grafting density. In contrast, when utilizing the "grafting from" method to fabricate polymer brushes, one can prepare dense brushes and control the grafting density by varying the initiator ratio. ${ }^{10}$ Through grafting polymers brushes, the substrate can be made suitable for various applications, e.g. to act as lubricants, ${ }^{11}$ create antibacterial surfaces, ${ }^{12}$ to allow for reversible cell attachment, ${ }^{13}$ to act as sensors, ${ }^{14}$ etc.

\subsection{Surface-initiated atom transfer radical polymerization (SI- ATRP)}

Using living polymerization, a variety of polymers can be grafted from the surface and the molecular weight can be precisely tailored. ${ }^{15}$ Frequently employed techniques ${ }^{16}$ for "grafting from" include (Surface-Initiated, SI) Atom Transfer Radical Polymerization (ATRP), Reversible Addition-Fragmentation Chain Transfer Radical Polymerization (RAFT), Initiator-Transfer-Terminator (INIFERTER) agent based polymerization and Nitroxide-Mediated Polymerization (NMP). Among them, SI-ATRP ${ }^{17}$ is the most extensively utilized. It was reported first in $1997 .{ }^{18}$ The general mechanism of ATRP ${ }^{15 d}$ is 
shown as in Scheme 2.1, which is based on an equilibrium between active radicals $\left(\mathrm{P}_{\mathrm{n}}{ }^{*}\right)$ and dormant alkyl halide terminated polymer chain ends $\left(\mathrm{P}_{\mathrm{n}}-\mathrm{X}\right)$. During the polymerization process, a transition metal catalyst ( $\mathrm{Mt}^{\mathrm{m}}$ represents the metal in oxidation state) and ligand (L) are used to periodically react with the dormant species at a rate constant of activation $\left(k_{\mathrm{act}}\right)$. The formed growing radicals $\left(\mathrm{P}_{\mathrm{n}}{ }^{*}\right)$ can propagate, which results in a polymerization of the monomers. Meanwhile, the transition metal complexes coordinate with halide ligand forms a higher oxidation state $\left(\mathrm{X}-\mathrm{Mt}^{\mathrm{m}+1} / \mathrm{L}\right)$. However, the formed deactivator can react with the active radicals in a reverse reaction to reform the dormant species and the activator (metal catalyst in a lower oxidation state). Since the dormant state is preferred in this equilibrium, each polymer chain will grow by only a few monomers at a time. Due to this low propagation rate, polymers of low polydispersity are grown. The main difference between SI-ATRP and ATRP in bulk is the extremely low concentration of initiators on the surface. ${ }^{19}$ After initiated, the concentration of deactivator is too low to trap the propagating radicals, which results in uncontrolled chain growth. ${ }^{20}$ Thus a relatively high concentration of deactivating $\mathrm{Cu}$ (II) is required in the recipe to establish the equilibrium between activate and inactivate chains during the SI-ATRP.

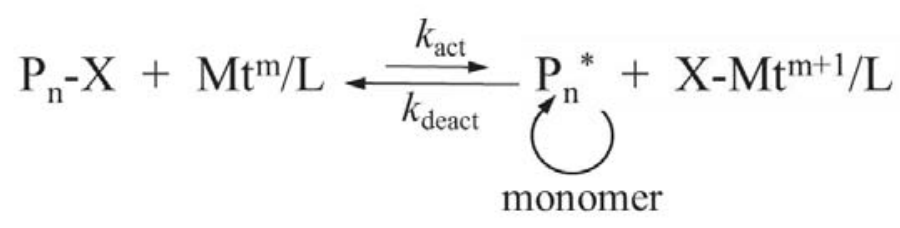

Scheme 2.1. Mechanism of ATRP

\subsection{Adhesion, adhesion hysteresis and friction}

The force of adhesion is defined as the force of attraction between different substances. ${ }^{21}$ When two solid surfaces are pressed together, they bond physically across the interface. The force needed to pull the two surfaces apart is called the adhesive force. Adhesion occurs both at solid-solid interfaces and between solid surfaces separated by a thin liquid film. ${ }^{22}$ In general, adhesion is high for clean and symmetric contacts, while contaminated contacts in many cases exhibit lower adhesion. ${ }^{22}$

Adhesion hysteresis is defined as the difference between the work needed to separate two surfaces and that originally gained on bringing them together. ${ }^{23}$ Also, it is referred to "Work" in some literature. ${ }^{24}$ The hysteresis is the energy cost which is needed to complete a contact and separation cycle of two surfaces. ${ }^{25}$

Friction is defined as 'the resistance that one surface or object encounters when moving over another'. ${ }^{26}$ Friction act opposite to the direction of motion. For solids in relative 
sliding motion, friction consists of an initial startup force (static friction force, $F_{\mathrm{s}}$ ), which under most circumstances arises by pinning due to adsorbed contaminants, ${ }^{27}$ and a kinetic (dynamic) friction force $F_{\mathrm{k}}$ at steady-state sliding. $F_{\mathrm{s}}$ is higher than or equal to the $F_{\mathrm{k}}$. In thermal equilibrium (e.g. in liquids), $F_{\mathrm{k}}$ increases linearly with velocity. ${ }^{28}$ Under these circumstances, we speak of Stokes friction. When the system is moved out-of-equilibrium (e.g. for solids), instabilities occur and $F_{\mathrm{k}}$ varies typically logarithmically with the velocity. ${ }^{28}$ For polymeric system, where the polymers can interdigitate and shear-align, more complex friction-velocity relations are found, ${ }^{29}$ especially when surfaces are rough. ${ }^{30}$

\subsection{Atomic force microscopy (AFM)}

Many types of equipment have been developed to measure the normal and lateral forces between two interacting surfaces, ${ }^{31}$ such as atomic force microscopy (AFM), ${ }^{9 b},{ }^{32}$ surface force apparatus (SFA) ${ }^{10,23 a, 33}$ and tribometer. ${ }^{34}$ AFM is a technique that is derived from scanning tunneling microscopy (STM), ${ }^{35}$ which can be used to visualize the surface topology of conductive surfaces down to atomic resolution. In 1986, Binnig and Quate demonstrated for the first time the employment of AFM in obtaining nanoscale resolution surface images. ${ }^{36}$ Compared to STM, now non-conductive materials (polymers, ceramic), in non-vacuous (air or liquid) environment can be imaged.

Since its invention, the AFM has evolved into a tool to characterize and manipulate structures and measure the interactions between surfaces with a nanoscale resolution. ${ }^{37}$ The working principle of the AFM is sketched in Figure 2.2. (a). The essential components of the AFM consist of a laser diode, cantilever, mirror, photodetector, piezo-electric scanner. During the measurement, a laser is reflected off the rear side of the cantilever. After another reflection by the mirror, the position of the laser beam is detected by a photodetector (4quadrant photo diode). Depending on the bending (up and down) of the cantilever, angular deflections can be detected. On the photodiode, the $(A+B)-(C+D)$ signal is proportional to the normal deflection of the cantilever, while the $(A+C)-(B+D)$ signal is a measure of the torsional force on the cantilever. After calibration of the cantilever spring constant, the deflection can be converted into a force. ${ }^{38}$ The AFM can be employed in different modes, such as contact mode (CM) AFM and tapping mode AFM. Both modes can be operated in air or liquid. Many groups apply AFM to measure the adhesion ${ }^{39}$ and friction ${ }^{40}$ between surfaces in relative motion.

Figure 2.2. (b) shows a typical adhesion measurement using AFM. When the sample gradually approaches the cantilever, either repulsive or attractive forces will bend the cantilever depending on the interaction between cantilever and sample. For attractive forces, a jump-to-contact occurs (blue sharp peak), when the derivative of the force is higher than the spring constant of the cantilever. Upon further approach, the piezo pushes the cantilever up on the sample surface. The cantilever bends upward, and a positive deflection is 
measured. Upon retraction, due to adhesive interaction, the cantilever cannot separate with sample at the original zero force position. With further retraction, the adhesion force results in a measured, negative deflection until the derivative of the force is less than the spring constant of the cantilever. The cantilever rapidly jumps back to its original position (red sharp peak). The maximum force needed to separate the cantilever and samples is called the adhesion force. The area between approach and retract curve equals the dissipated energy in the process, which is the adhesion hysteresis. There are various other AFM techniques next to contact mode to measure adhesion (hysteresis), such as HarmoniX, ${ }^{41}$ peak force, ${ }^{41 a}, 41 \mathrm{~d}, 42$ noise analysis ${ }^{43}$ and others. ${ }^{44}$
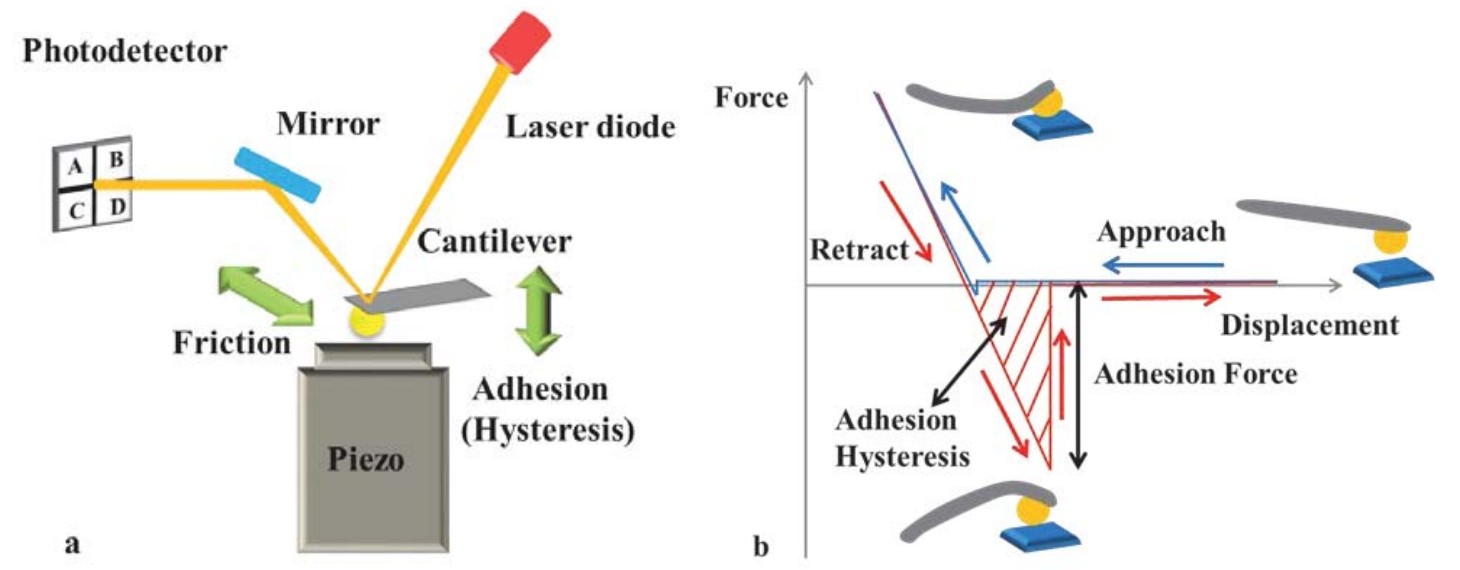

Figure 2.2. (a) Schematic diagram showing the working principle of AFM and (b) an illustration of a force versus $\mathrm{z}$ piezo displacement curve.

AFM has also been used extensively to measure the friction between surfaces in relative sliding motion. ${ }^{45}$ For friction measurements, the sample surface is slid in the lateral direction. Due to the friction force, the cantilever will twist in the torsional direction. After calibration of the torsional spring constant and the deflection sensitivity, ${ }^{38,45 \mathrm{~b}}$ the friction force can be calculated from the signal of the quadrant photodetector.

\subsection{Stimulus responsive polymers}

Stimulus responsive (SR) polymers, are polymers that adapt their physicochemical properties in response to modifications in the environmental conditions, such as temperature, ${ }^{32 \mathrm{e},}{ }^{46} \mathrm{pH},{ }^{47}$ ionic concentration of the solvent, ${ }^{47 \mathrm{i}, 48}$ effective solvent conditions by addition of cosolvents/co-non-solvents, ${ }^{9 b}, 32 f, 32 \mathrm{~g},{ }^{49} \mathrm{UV}$-vis light irradiation, ${ }^{50}$ redox $^{51}$ or the presence and strength of electronic and magnetic fields ${ }^{52}$ (see also Figure 2.3). With these external stimuli, the chemical structure ${ }^{53}$ or the conformation of these polymers can 
be changed. ${ }^{54}$ We note that, within this definition, all polymers will be SR polymers under some conditions. However, we speak of SR polymers when they respond to the specific stimulus that is applied in the discussed experiment or potential application. If the response of the SR polymers to the stimulus is reversible, and the physico-chemical properties can be switched repeatedly. ${ }^{32 a, 55}$

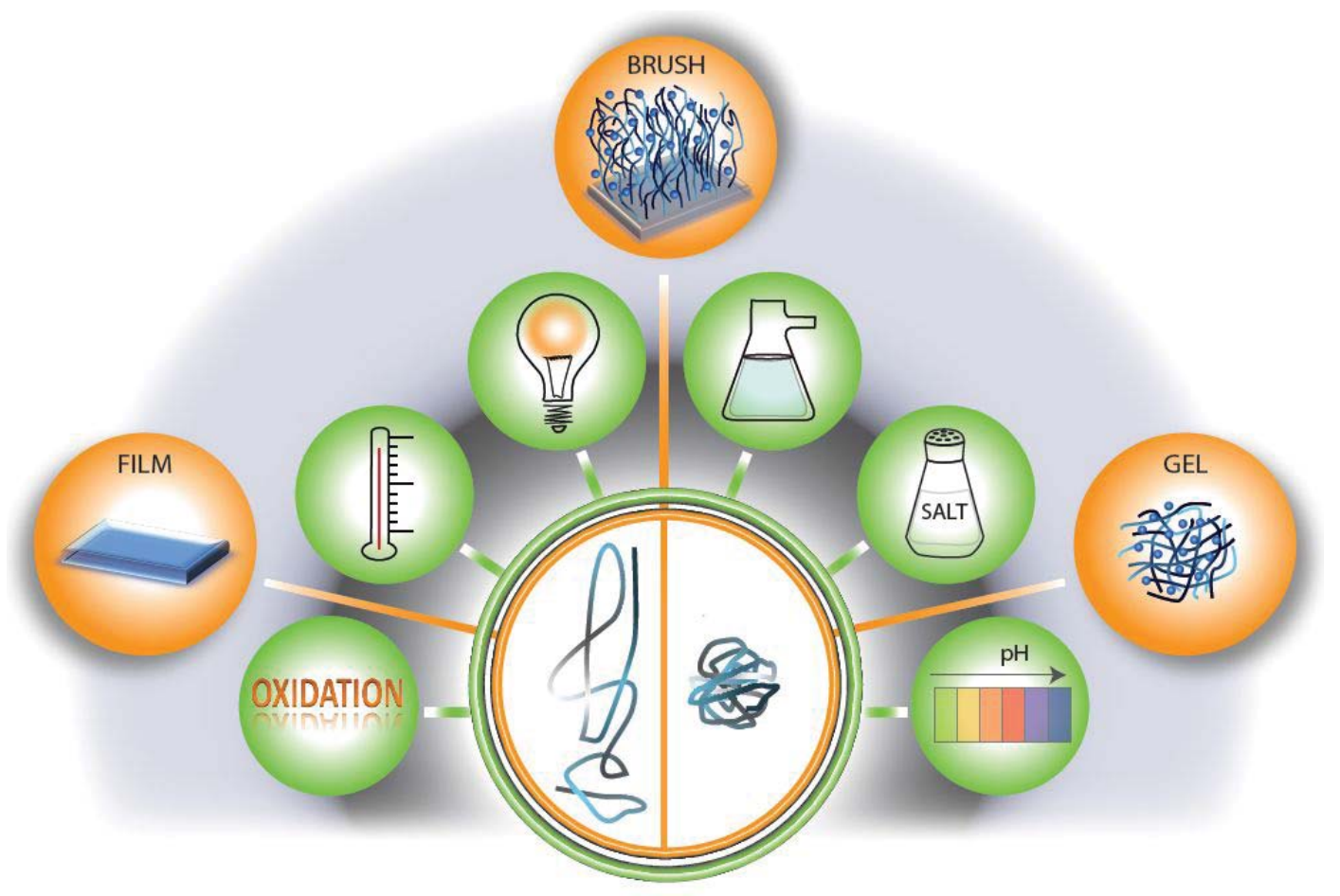

Figure 2.3. Schematic diagram of stimulus responsive polymers in gels, films and brushes triggered by various external stimuli.

\subsubsection{Temperature}

Thermally responsive polymers can have a lower critical solution temperature (LCST) and/or an upper critical solution temperature (UCST). UCST behavior can be understood via the Flory-Huggins mean-field theory. ${ }^{2 b}$ Upon increasing the temperature the FloryHuggins parameter $\chi$ reduces. Therefore, the entropic mixing contribution to the free energy will dominate over the enthalpy at high temperatures and, consequently the components mix. LCST behavior is, however, not captured by the standard Flory-Huggins theory. ${ }^{56}$ The reason for this is that the interactions between sub-units within $\chi$ are considered to be independent of the temperature and volume fraction within the Flory-Huggins theory, which is a simplification of realistic interactions. Using a modified equation for the interaction parameter $\chi$, the interaction energy can be made temperature and volume 
fraction dependent, ${ }^{57}$ which allows for predicting both the UCST and LCST in polymersolvent mixtures. ${ }^{58}$ Various models have been proposed to explain the temperature and/or volume fraction dependent interaction energies for different polymer-solvent combinations. ${ }^{59}$ A well-studied example is poly( $N$-isopropyl acrylamide) (PNIPAM), which exhibits an LCST close to room temperature (typically $30-33{ }^{\circ} \mathrm{C}$ ) 60 in pure water. ${ }^{61}$ For this polymer, the most common explanation for temperature-dependent interactions is that, below the LCST, the enthalpy-gain from hydrogen bonds between PNIPAM and $\mathrm{H}_{2} \mathrm{O}$ outweighs the reduction in entropy caused by water-adsorption. Above the LCST, the increased entropy of the system increases the entropy-reduction due to adsorption such that adsorption is no longer energetically favorable. At these higher temperatures, the wateramide hydrogen bonds are replaced by amide-amide hydrogen bonds between the polymer segments, which results in phase separation.

\subsubsection{Co-(non-)solvency}

Cosolvency and co-non-solvency are generic phenomena ${ }^{61 \mathrm{a},} 62$ that occur for a variety of polymers and in different solvent mixtures. Cosolvency is the effect that a mixture of two poor solvents can become a good solvent for a polymer at certain relative volume fractions. This effect can be understood qualitatively via the Flory-Huggins theory ${ }^{56}$ using the single liquid approximation ${ }^{63}$ : The effective interaction parameter $\chi$ between the polymer $\mathrm{P}$ and solvent mixture $(\mathrm{S} 1, \mathrm{~S} 2)$ is defined as:

$\chi=\phi_{1} \chi_{\mathrm{PS} 1}+\phi_{2} \chi_{\mathrm{PS} 2}-\phi_{1} \phi_{2} \chi_{\mathrm{S} 1 \mathrm{~S} 2}$

in which $\phi$ is the solvent volume fraction. Since both individual solvents are poor, $\chi_{\mathrm{PS} 1}$ and $\chi_{\mathrm{PS} 2}>0.5$. Additionally, the two solvents are miscible and $\chi_{\mathrm{S} 1 \mathrm{~S} 2}$ can be just lower than 2 for particular solvents. When substituting these numbers in formula 1 , one can see that $\chi$ can be less than 0.5 for certain solvents and $\phi$, which shows that the mixture can be a good solvent for the polymer. For example, both water and ethanol are poor solvents for poly(methyl methacrylate) (PMMA). However, for a volume fraction of $80 \%$ ethanol in water, the mixture becomes a good solvent for PMMA both in a bulk solution ${ }^{62 a}$ and in the gelform. ${ }^{62 b}$

For co-non-solvency, the opposite will happen: When two good solvents are mixed, the mixture can be a poor solvent for the polymer. The mechanism for co-non-solvency is still under debate, and many theories and models are proposed to explain the phenomenon, such as competition of forming hydrogen bonds between alcohol and water with PNIPAM, ${ }^{64}$ the formation of composition-dependent solvent-clusters ${ }^{65}$ and the bridge model. ${ }^{61 \mathrm{~b},} 66$ The most well-known example of co-non-solvency is PNIPAM in water and an organic solvent, such

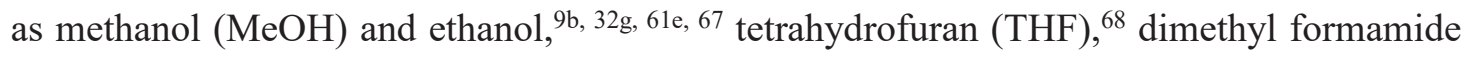
(DMF), ${ }^{69}$ dimethyl sulfoxide (DMSO). ${ }^{70}$ Both cosolvency and co-non-solvency effects have potential application in actuator, ${ }^{71}$ gating $^{72}$ and pick up and release systems ${ }^{32 f}$ in liquid environment. 


\subsection{3 $\mathrm{pH}$ and salt concentration}

For polyelectrolytes, such as polycations, ${ }^{47 \mathrm{~g},} 47 \mathrm{~h}, 73$ polyanions,${ }^{74}$ and zwitterionic ${ }^{75}$ polymers, the $\mathrm{pH}$ can be used as an external stimulus. A typical example is polyacrylic acid (PAA), ${ }^{47 a}$ which is a polyacid with carboxyl groups on the side chain. The addition of a base will deprotonate the pendant acidic groups, such that charges are introduced within the chain. Consequently, it swells. In an acidic solution, protonation of carboxyl group causes a chaincollapse. Moreover, water-soluble salts also can be used to induce a coil-to-globule transition of polyelectrolytes, due to the change in electrostatic interactions. ${ }^{76}$ Also, the solubility of zwitterionic polymers is sensitive to the salts concentration. For example, for poly[2-(methacryloyloxy)ethyl]-dimethyl(3-sulfopropyl) ammonium hydroxide (PSBMA) ${ }^{77}$ adding salts helps to reduce the intra-chain interaction of zwitterionic moiety, which increases the swelling ratio in water.

\subsubsection{Other and multi-stimuli}

Other examples of SR polymers are polymers functionalized with light-responsive groups, such as azobenzene, ${ }^{78}$ spiropyran $^{50 a}$ and coumarin. ${ }^{79}$ For example, azobenzene can switch between trans- and cis- conformation when irradiated by visible light and UV light. The trans- and cis- conformation have different energies and molecular geometries, such that an incorporation of the groups in polymers can induce a change in the effective interaction with itself and other molecules upon photo-irradiation. ${ }^{80}$

For redox-responsive polymers, reversible oxidation-reduction reactions are employed to induce a change in the effective interactions of the polymer with the surrounding medium. There are many functional groups showing oxidization-reduction dependent properties, such as ferrocene, ${ }^{81}$ tetrathiafulvalene, ${ }^{82}$ conjugated groups, ${ }^{83}$ transition metal ions,${ }^{84}$ disulfides. ${ }^{85}$ The typical example is the ferrocene functionalized polymer, which has a redox responsive center located in the polymer's main chain ${ }^{51 \mathrm{a}, 51 \mathrm{e}}$ or side chain. ${ }^{55 \mathrm{c},}{ }^{86}$ After oxidization, ferrocene exhibits charged ferricenium moieties, which interact electrostatically with counterions. ${ }^{87}$

By a modification of stimulus responsive polymers with responsive functional groups one can build polymers that respond to multiple types of stimuli. ${ }^{88}$ When stimulus responsive polymers are covalently attached to surfaces in the form of gels, films or brushes, they can be employed to induce macroscopic changes in the surface or interfacial properties, for example from wetting to non-wetting, ${ }^{53}$ from soluble to non-soluble, ${ }^{46 \mathrm{e}}$ from adhering to non-adhering, ${ }^{54}$ from lubricating to non-lubricating. ${ }^{89}$ The latter two will be explained in more detail below. 


\subsection{Switchable adhesion}

When a gecko walks over the ceiling, it needs to frequently attach and detach its feet. Mimicking such switchable adhesion as found in nature has enormous potential for application. For example, in the DARPA's Z-Man program, climbing devices are being developed that mimic geckos. With these hand-hold devices, a climber that weights around $100 \mathrm{~kg}$ can ascend and descend freely on glass. Nowadays, many research groups develop polymeric materials that will respond to different stimuli ${ }^{9 b}$, 32a, 32e-g, 46c, 51b, 51e, 55, 87, 90 such that switchable adhesion can be applied under different circumstances. The schematic representations of interaction between chemically identical polymer films or with chemically different counter surfaces are shown in Figure 2.4-2.5. An overview of the research so far is given in Table 2.1. The abbreviations can be found at the end of this chapter.

\subsubsection{Adhesion between chemically identical polymer films}

The adhesion between chemically identical polymer films depends on the solvent quality. In good solvent, polymers are effectively repulsive and, thus, direct polymer-polymer interactions are screened. When two polymeric systems in good solvent are pressed together, the solvent is kept in the contact unless the applied pressure is higher than the osmotic pressure in the solvent. ${ }^{91}$ The adhesion is generally low under such mildcompression conditions. ${ }^{32 e,} 92$ In poor solvents, direct polymer-polymer interactions are preferred and under these circumstances adhesion between the polymer films can be high. ${ }^{10}$ ${ }^{32 f}$ Thus, when the applied stimulus changes the effective solvent quality for the polymer films from good to poor, the adhesion can be altered from low to high. For example, Malham et al. employed temperature to change the adhesion between PNIPAM and PNIPAM brushes on mica surfaces by using SFA, and the magnitude of adhesion can be tuned by at least 20 times. Also, co-non-solvency can be used to switch the adhesion between PNIPAM gels and brushes. ${ }^{55 \mathrm{a}}$

An exception to this generic behavior can be found for systems where the polymers in solvated polymer films in close contact can entangle ${ }^{55 a}, 103$ as e.g. for two brushes in close contact or for brushes in contact with gels. As reported, the typical degree of polymerization above which there can be entanglements in brushes is $3000 .{ }^{103}$ Normally, the number of repeat units of polymers in brushes are less than that number, ${ }^{32 \mathrm{e}}$ such that the macromolecules chains will only interdigitate. ${ }^{32 \mathrm{c}}$ With increase of the waiting time ${ }^{104}$ and grafting density, ${ }^{105}$ the interpenetration of opposing brush layers also increase, which also might cause entanglement at lower degrees of polymerization. ${ }^{106}$ Brushes with interdigitated polymers can easily be separated, such that the adhesion is low. ${ }^{107}$ When both polymer films are cross-linked and form a (hydro-)gel, there will be no entanglements and, therefore the adhesion between two solvated hydrogels is always low, while the adhesion between collapsed hydrogels is high. ${ }^{108}$ This was, for example, shown by Banquy et al., ${ }^{108}$ 
who measured that the adhesion between two PDEA gels can be changed 17 times in magnitude, altering the temperature between $15^{\circ} \mathrm{C}$ and $30^{\circ} \mathrm{C}$. The same results were found for brushes in contact with gels, ${ }^{55 a}$ which means that the polymers in these brushes were too small to entangle.

Table 2.1. Switchable adhesion by various stimuli

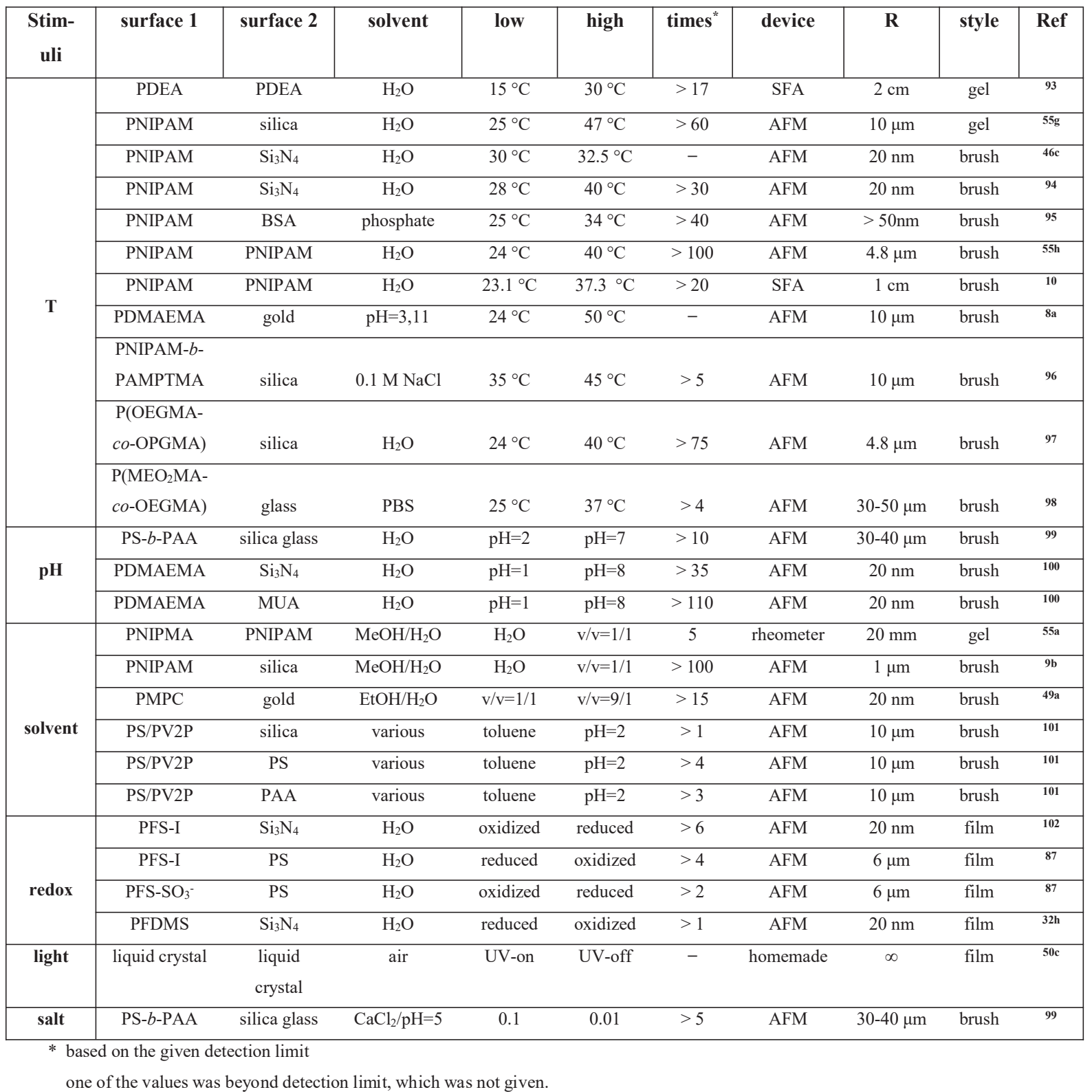




\subsubsection{The adhesion between polymer films and chemically different counter surfaces}

To predict the adhesion between a polymer film and a chemically different counter surface (CS) is more complicated than contacts between chemically identical polymer films, since the polymer-CS interactions play a crucial role in this. When the polymer-CS interactions are stronger than the interactions between the polymer and the solvent, and the counter surface and the solvent, adhesion can be high, even when solvent conditions are good. ${ }^{32 \mathrm{e}}$ The change in adhesion is then determined by the variation in contact area due to the alteration in effective elasticity of the brush. For example, Vyas et al. ${ }^{101}$ used AFM to measure the adhesion under good solvent conditions between silicon sharp cantilever and PS brush was high, while the adhesion between silica colloid cantilever and PS brush was low. Moreover, Raftari et al. ${ }^{100}$ employed gold cantilever with different coatings to investigate the adhesion of PDMAEMA brushes at $\mathrm{pH} 8$ solution, such as mercaptoundecanoic acid (MUA) or dodecanethiol (DDT) coatings. The adhesion between MUA-coated cantilever and brush is around $12 \mathrm{nN}$, while DDT-coated for $4 \mathrm{nN}$.

Some polymers are, however, special in the sense that they very strongly interact with particular solvents and, therefore, repel almost any counter surface in that solvent. These polymers are considered to be so-called non-fouling polymers. The low-fouling properties can only be achieved under particular solvent-conditions, such that also for these systems adhesion can be switched by changing the effective solvent quality. For example, PMPC is well-known to be an anti-fouling material in aqueous solution to resist bacteria ${ }^{109}$ and protein. ${ }^{110}$ Yang et al. ${ }^{49 a}$ used QCM to show that in water there was high adherence between proteins and PVBIPS brushes, while by adding $1 \mathrm{M}$ sodium chloride $(\mathrm{NaCl})$ solution, the surface hydration of the brushes could be enhanced to give a low adherence and the serum and plasma could be completely washed out.

For some polymers it is possible to directly change the interaction between the polymer and the counter surface. ${ }^{32 \mathrm{~h}, 51 \mathrm{a}, 5 \mathrm{~b}}$ For example, poly(ferrocenylsilane) (PFS) is composed of ferrocene unit in the main chain, which can be chemically or electrochemically reduced and oxidized reversibly. ${ }^{51 a}$, 51e, 51f, 87, 111 This can be employed to change the adhesion directly without the mediation of the solvent. For example, Feng et al. ${ }^{112}$ grafted PFS-I on Au surface, and the $\mathrm{Si}_{3} \mathrm{~N}_{4}$ probe was treated with organic solvent and piranha solution separately. In both cases, the adhesion could be tuned before and after oxidation. However, opposite trends on switching adhesion were found using these two different probes: a piranha treated probe has a negative charge, while a solvent treated probe is neutral. Thus different interfacial interactions were obtained under oxidation and reduction state. 


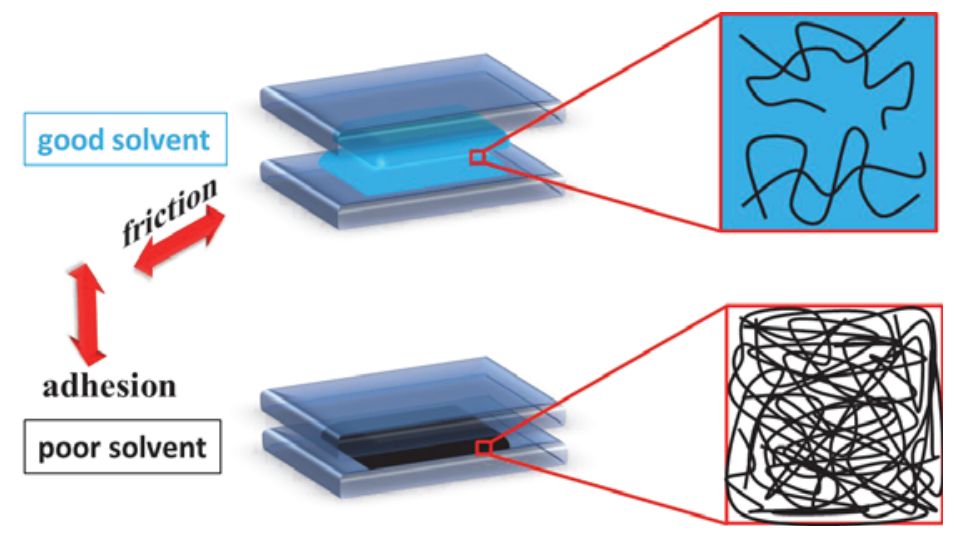

Figure 2.4. Schematic representation of adhesion and friction between chemically identical polymer films in good or poor solvents. The polymers on the substrates could be in the form of gels, films or brushes.

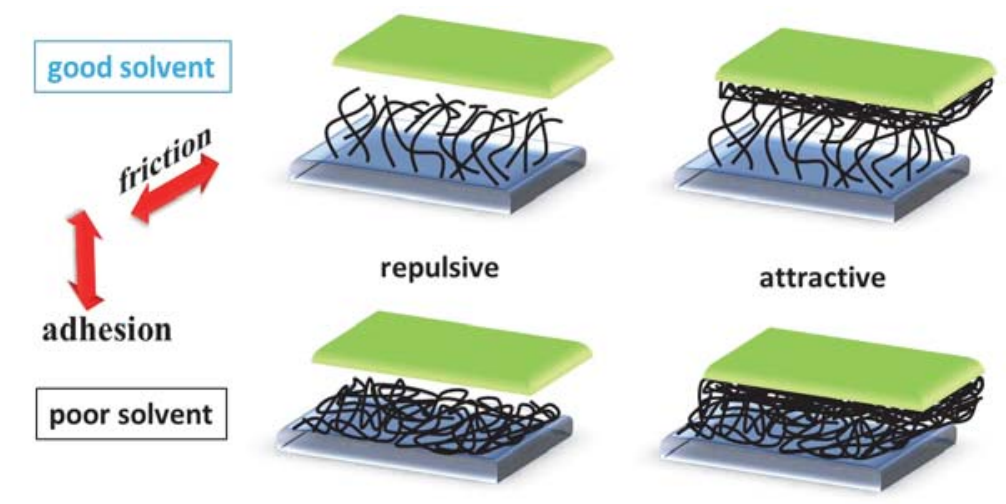

Figure 2.5. Schematic representation of adhesion and friction between polymer films and chemically different counter surfaces in good or poor solvents for repulsive and attractive interactions between the walls and polymers. The polymers on the substrates could be in the form of gels, films or brushes.

\subsection{Switchable friction}

Switchable friction can find many applications in tissue engineering and biomedical systems, ${ }^{34 a}$, 34c, 34d, 48g, 55a such as contact lenses, artificial cartilage, catheter etc. In these systems, objects should be able to resist sliding after being positioned, but should nevertheless be easy to apply within the patient. Moreover, switchable friction is required e.g. in order to let robots walk on walls. ${ }^{50 \mathrm{c}, 50 \mathrm{~d}}$ Polymeric systems can fit these requirements and in recent years, there have been many attempts to achieve such switchable friction ${ }^{23 a}$, 32a, 34a, 34c, 34d, 48g, 55a, 101, 113 (see also Table 2.2). 


\subsubsection{Friction between chemically identical polymer films}

The presence of interdigitation between macromolecules of opposing polymeric systems strongly determines the alteration in friction upon applying an external stimulus. In the absence of interdigitation, friction is determined by polymer-polymer interactions in the contact. In a good solvent, direct polymer-polymer interactions are screened and therefore, the solvent-(viscosity) determines friction and, therefore, friction is low. ${ }^{11 a}$ In poor solvents, polymer-polymer interactions are favored over polymer-solvent interactions and under these conditions friction is high. ${ }^{34}$ These conditions can be obtained for brushes or hydrogels under low normal loads. The friction under these circumstances can be switched from high to low by changing the effective solvent quality from poor to good. For example, $\mathrm{Wu}$ et al. ${ }^{34 \mathrm{c}}$ used temperature to switch the friction between PNIPAM/GO gels by changing the temperature between 28 and $38{ }^{\circ} \mathrm{C}$, and the friction could be changed more than 10 times in magnitude. Moreover, Liu et al. ${ }^{50 \mathrm{c}}$ made azobenzene-based liquid crystal smart coatings. By turning on and off UV light, the 3-D fingerprint structure could be modulated to tune the interfacial friction.

Under high normal loads, opposing polymer brushes can interdigitate, ${ }^{32 \mathrm{c}}$ which results in high friction. ${ }^{33 b, 33 c}$ Upon sliding the interdigitated opposing polymer brushes, the polymers tilt, ${ }^{105}$ resulting in a decrease of the overlap-zone or interdigitation for higher shear-rates. Consequently, there is a sublinear friction-velocity relation ${ }^{114}$ for interdigitated polymer brushes. Under these circumstances, friction can be higher than for collapsed brushes in a poor solvent, due to the larger effective contact area in the latter. ${ }^{32 f}$ Therefore, these systems can respond oppositely to the applied stimulus than non-interdigitating polymeric systems. $^{32 \mathrm{~d}}$

\subsubsection{Friction between polymer films and chemically different counter surfaces}

The friction between polymer films and chemically different solids is determined by the solvent-mediated polymer-solid interactions. If polymer-solid interactions are higher than polymer-solvent and solid-solvent interactions, the friction change is determined by the change in contact are upon applying the stimulus. If the solvent screens direct polymersolid interactions under good solvent conditions, friction is low and can be switched to be high by applying a stimulus that changes the solvent conditions to poor. Zhang et al. ${ }^{49 a}$ measured the friction between gold cantilever and PMPC brushes. The coefficient of friction (COF) was determined by friction force microscopy (FFM) in various ratio of water/EtOH. The excellent lubrication properties of PMPC brushes started to reduce from $70 \%$ of EtOH volume fraction due to the collapse of PMPC. The highest COF was found at 90\% EtOH content, where PMPC brushes are completely collapsed. When the volume of $\mathrm{EtOH}$ is $100 \%$, again a low friction coefficient is observed. However, directly opposite results are found by Kobayashi et al. ${ }^{115}$ using a tribotester. Their explanation is that swollen 
brushes have more effective contact areas than that of collapsed brushes. Moreover, Wei et $a l .{ }^{34 a}$ systematically investigated the switchable friction of polyelectrolyte brushes. For polycation, the friction could be tuned by simply changing the type of counterion, which had specific ion effect as the following order $\mathrm{Cl}^{-}<\mathrm{ClO}_{4}^{-}<\mathrm{PF}_{6}{ }^{-}<\mathrm{TFSI}^{-}$, which is also the hydrophobicity order. More hydrophobic counterions offer more hydrophobicity of the corresponding polymer brushes, thus the brushes collapse more. The higher friction is due to a more energy dissipation in dehydrated and collapsed brushes. For polyanionic brushes, the frictional response to sliding can be tuned by surfactants with various length of hydrophobic tails due to the electrostatic interaction. They conclude that hydrated and swollen polyelectrolyte brushes can form excellent lubricants, while, dehydrated and collapsed brushes behave less ideal lubrication properties.

A special kind of switchable friction can be obtained by grafting two different types of polymers to two surfaces. ${ }^{90 \mathrm{~b},}{ }^{101}$ When the different types of polymers swell in different solvents and interact differently with the counter surface, friction can be switched by solvent exchange. For example, de Beer et al. ${ }^{90 \mathrm{~b}}$ used AFM to measure the friction between PMMA coated gold colloid and PNIPAM coated substrate by immersing in two different solvents (acetophenone for PMMA and water for PNIPAM). Ultralow friction was obtained in this asymmetric system rather than the symmetric system in only one solvent, shown in Figure 2.6.

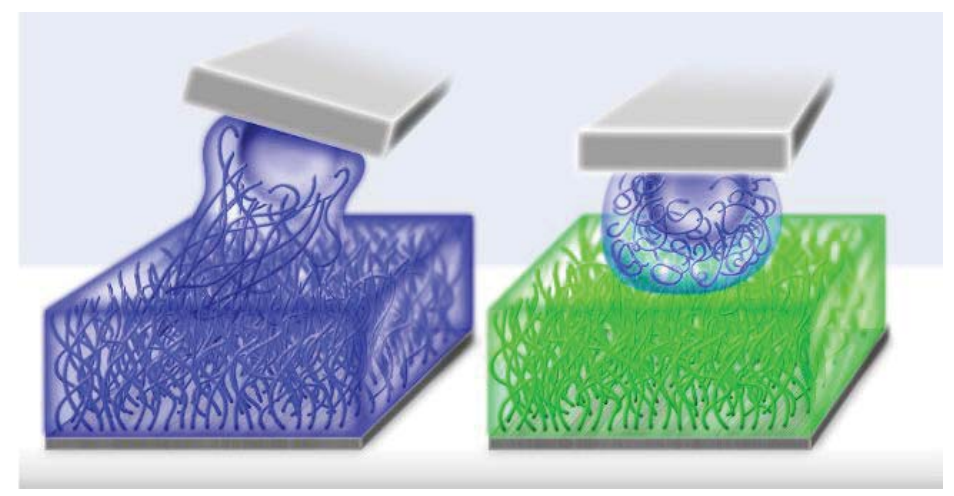

Figure 2.6. Schematic sketch of the symmetric and asymmetric brushes contact in the same and different solvents. Left panel shows the miscible system, where the same polymers are grafted from the surface and the colloid. The brushes are solvated in a one-phase liquid. The right panel shows the immiscible system of two different polymer brushes. Each brush is solvated in its own preferred liquid. In traditional miscible systems, the polymers of the opposite brushes overlap. For the immiscible system, opposite brushes do not interdigitate such that friction and wear during sliding are reduced. ${ }^{32 \mathrm{~b}}$

Particular types of asymmetric contacts are contacts between two chemically different polymer brushes. When pressures are high and both brushes absorb the same solvent, 
friction is high due to interdigitation between the opposing brushes. If, however, each brush prefers their own solvent, polymers are kept in their own brush and interdigitation is strongly reduced. ${ }^{90 \mathrm{~b}}$ If one of the two opposing brushes responds to an external stimulus, friction can be made switchable. ${ }^{32 a}$ For example, if in one state both brushes prefer the same solvent, friction is high due to interdigitation. After applying the stimulus, one of the brushes will expel the solvent and the brushes no longer interdigitate such that friction is low provided that the solvent-polymer interactions in the swollen brush are higher than the polymer-polymer interactions.

\subsection{Relation between friction and adhesion/adhesion hysteresis}

The question if adhesion and friction are directly related has triggered many scientific studies in the last decades. ${ }^{33 a}$, 120 The answer to this question is not generic, but instead depends on the particular system that is being studied. ${ }^{29 \mathrm{~d}}$ From a mathematical perspective, two perfectly flat walls without any corrugation would not resist sliding motion even when adhesion is high. ${ }^{121}$ Of course, these mathematical surfaces do not exist in realistic

applications. Real surfaces have at least an atomic corrugation, which could resist sliding motion when the corrugations of the opposing surfaces are commensurate. Nevertheless, any degree of mismatch between the surfaces would induce incommensurability such that ratio of friction to adhesion vanishes. ${ }^{122}$ For macroscopic engineering surfaces, such as in sliding metal surfaces in the absence of wear, friction and adhesion are often found to be directly related: Variation of external conditions can lead to a reduction or increase in both friction and adhesion. ${ }^{123}$ For example, Autumn et al. ${ }^{124}$ tested the relation between adhesion and shear force in isolated setal arrays and live gecko toes. A linear relation is found between adhesion and shear force. Moreover, Chen et al. ${ }^{125}$ studied the friction force and adhesion between two PS and poly(vinylbenzyl chloride) surfaces by SFA. After crosslinking of the polymer surfaces, both friction and adhesion are reduced. While adding chain ends causes the increase of friction and adhesion. On the other hand, some experimental counter-examples have also been reported. ${ }^{126}$ For example, two smooth mica surfaces separated by molecular layers of cyclohexane exhibit high COF, but a low adhesion energy. In contrast, mica surfaces in humid air exhibit low COF, but high adhesion energy due to capillary forces. Thus, only when friction and adhesion (hysteresis) are caused by the same interactions/dissipation mechanisms they can be directly related (even when prefactors can be directional dependent). ${ }^{127}$ In the following paragraph, we will focus on discussing the relationship between friction and adhesion in stimulus-response polymer films in contact. 
Table 2.2. Switchable friction by various stimuli

\begin{tabular}{|c|c|c|c|c|c|c|c|c|c|c|}
\hline $\begin{array}{c}\text { Stim- } \\
\text { uli }\end{array}$ & surface 1 & surface 2 & solvent & low & high & times $^{*}$ & device & $\mathbf{R}$ & style & Ref \\
\hline \multirow{9}{*}{$\mathbf{T}$} & PDEA & PDEA & $\mathrm{H}_{2} \mathrm{O}$ & $15^{\circ} \mathrm{C}$ & $30^{\circ} \mathrm{C}$ & $>6$ & SFA & $2 \mathrm{~cm}$ & gel & 93 \\
\hline & PDMAEMA & gold & $\mathrm{pH}=3,11$ & $50^{\circ} \mathrm{C}$ & $24^{\circ} \mathrm{C}$ & $>1$ & AFM & $10 \mu \mathrm{m}$ & brush & $8 \mathrm{a}$ \\
\hline & $\begin{array}{l}\text { PNIPAM-b- } \\
\text { PAMPTMA }\end{array}$ & silica & $0.1 \mathrm{M} \mathrm{NaCl}$ & $25^{\circ} \mathrm{C}$ & $35^{\circ} \mathrm{C}$ & $>4$ & AFM & $10 \mu \mathrm{m}$ & brush & 96 \\
\hline & $\begin{array}{l}\text { PDMAEMA- } \\
\text { stat-BPMA }\end{array}$ & $\mathrm{Si}_{3} \mathrm{~N}_{4}$ & $\mathrm{H}_{2} \mathrm{O}$ & $25 / 60^{\circ} \mathrm{C}$ & $45^{\circ} \mathrm{C}$ & $>1$ & AFM & $20 \mathrm{~nm}$ & gel & 116 \\
\hline & PNIPAM/GO & PNIPAM/GO & $\mathrm{H}_{2} \mathrm{O}$ & $28^{\circ} \mathrm{C}$ & $36^{\circ} \mathrm{C}$ & $>10$ & tribometer & $35 \mathrm{~mm}$ & gel & $34 \mathrm{c}$ \\
\hline & PNIPAM- & PNIPAM- & & $\mathrm{pH}=7$ & $\mathrm{pH}=2$ & & & & & \\
\hline & NaMA & NaMA & phosphate & $\mathrm{rt}$ & $32^{\circ} \mathrm{C}$ & $>20$ & tribometer & $35 \mathrm{~mm}$ & gel & 117 \\
\hline & PNIPAM- & PNIPAM- & & $\mathrm{pH}=2$ & $\mathrm{pH}=8$ & & & & & \\
\hline & DMAEMA & DMAEMA & phosphate & $\mathrm{rt}$ & $30^{\circ} \mathrm{C}$ & $>14$ & tribometer & $35 \mathrm{~mm}$ & gel & 117 \\
\hline \multirow{3}{*}{ pH } & PDMAEMA & gold & $\mathrm{H}_{2} \mathrm{O}$ & $\mathrm{pH}=3$ & $\mathrm{pH}=11$ & $>10$ & AFM & $10 \mu \mathrm{m}$ & brush & $8 \mathrm{a}$ \\
\hline & PDMAEMA & $\mathrm{Si}_{3} \mathrm{~N}_{4}$ & $\mathrm{H}_{2} \mathrm{O}$ & $\mathrm{pH}=1$ & $\mathrm{pH}=8$ & $>12$ & AFM & $20 \mathrm{~nm}$ & brush & 100 \\
\hline & PMAA & PDMS & $\mathrm{H}_{2} \mathrm{O}$ & $\mathrm{pH}=7$ & $\mathrm{pH}=2$ & $>300$ & tribometer & $6 \mathrm{~mm}$ & brush & $34 a$ \\
\hline \multirow{7}{*}{ solvent } & PNIPAM & PNIPAM & $\mathrm{MeOH} / \mathrm{H}_{2} \mathrm{O}$ & $\mathrm{H}_{2} \mathrm{O}$ & $\mathrm{v} / \mathrm{v}=1 / 1$ & $>6$ & rheometer & $20 \mathrm{~mm}$ & gel & $55 a$ \\
\hline & PMPC & gold & $\mathrm{EtOH} / \mathrm{H}_{2} \mathrm{O}$ & $\mathrm{v} / \mathrm{v}=1 / 1$ & $\mathrm{v} / \mathrm{v}=9 / 1$ & $>9$ & AFM & $20 \mathrm{~nm}$ & brush & $49 \mathrm{a}$ \\
\hline & PS/PV2P & silica & various & toluene & $\mathrm{pH}=2$ & $>1$ & AFM & $10 \mu \mathrm{m}$ & brush & 101 \\
\hline & PS/PV2P & PS & various & toluene & $\mathrm{pH}=2$ & $>2$ & AFM & $10 \mu \mathrm{m}$ & brush & 101 \\
\hline & PS/PV2P & PAA & various & toluene & $\mathrm{pH}=2$ & $>5$ & AFM & $10 \mu \mathrm{m}$ & brush & 101 \\
\hline & PNIPAM & silica & $\mathrm{MeOH} / \mathrm{H}_{2} \mathrm{O}$ & $\mathrm{H}_{2} \mathrm{O}$ & $\mathrm{v} / \mathrm{v}=1 / 1$ & $>4$ & AFM & $1 \mu \mathrm{m}$ & brush & $32 \mathrm{~g}$ \\
\hline & PMPC & glass & $\mathrm{EtOH} / \mathrm{H}_{2} \mathrm{O}$ & $\mathrm{v} / \mathrm{v}=17 / 3$ & $\mathrm{EtOH}$ & $>1.5$ & tribometer & $10 \mathrm{~mm}$ & brush & 115 \\
\hline \multirow[t]{2}{*}{ redox } & PFDMS & $\mathrm{Si}_{3} \mathrm{~N}_{4}$ & $\mathrm{H}_{2} \mathrm{O}$ & reduced & oxidized & $>1$ & AFM & $20 \mathrm{~nm}$ & film & $32 \mathrm{~h}$ \\
\hline & PFDMS & $\mathrm{Si}_{3} \mathrm{~N}_{4}$ & $\mathrm{H}_{2} \mathrm{O}$ & $\mathrm{NaNO}_{3}$ & $\mathrm{NaClO}_{4}$ & $>1$ & AFM & $20 \mathrm{~nm}$ & film & $32 \mathrm{~h}$ \\
\hline light & liquid crystal & liquid crystal & air & UV-on & UV-off & $4-5$ & homemade & $\infty$ & film & 50c \\
\hline \multirow{9}{*}{ salt } & PMAA & PDMS & $\mathrm{H}_{2} \mathrm{O}$ & $\mathrm{Na}^{+}$ & CTAB & $>300$ & tribometer & $6 \mathrm{~mm}$ & brush & $34 \mathrm{a}$ \\
\hline & PMETAC & PDMS & $\mathrm{H}_{2} \mathrm{O}$ & $\mathrm{Cl}^{-}$ & TFSI $^{-}$ & $>200$ & tribometer & $6 \mathrm{~mm}$ & brush & $34 a$ \\
\hline & PSPMA & PDMS & $\mathrm{H}_{2} \mathrm{O}$ & $\mathrm{K}^{+}$ & CTAB & $>50$ & tribometer & $6 \mathrm{~mm}$ & brush & $34 a$ \\
\hline & $\begin{array}{c}\text { P(METAC)- } \\
b-(\text { PEO45M } \\
\text { EMA })\end{array}$ & $\begin{array}{c}\text { P(METAC)- } \\
b-(\text { PEO45M } \\
\text { EMA })\end{array}$ & $\mathrm{H}_{2} \mathrm{O}$ & $\mathrm{H}_{2} \mathrm{O}$ & $\mathrm{NaCl}$ & $>2$ & AFM & $20 \mu \mathrm{m}$ & brush & 118 \\
\hline & PSPMA & PDMS & CTAB & $\mathrm{H}_{2} \mathrm{O}$ & $0.95 \mathrm{mM}$ & $>30$ & tribometer & $5 \mathrm{~mm}$ & brush & 119 \\
\hline & PMETAC & PDMS & SDS & $\mathrm{H}_{2} \mathrm{O}$ & $>0.1 \mathrm{mM}$ & $>150$ & tribometer & $5 \mathrm{~mm}$ & brush & 119 \\
\hline & PVBIPS & PDMS & $\mathrm{NaCl}$ & $6.1 \mathrm{M}$ & $\mathrm{H}_{2} \mathrm{O}$ & $>15$ & tribometer & $6 \mathrm{~mm}$ & brush & $48 \mathrm{~g}$ \\
\hline & PVBIPS & PDMS & $\mathrm{H}_{2} \mathrm{O}$ & $\mathrm{Br}^{-}$ & $\mathrm{SO}_{4}^{2-}$ & $>30$ & tribometer & $6 \mathrm{~mm}$ & brush & $48 \mathrm{~g}$ \\
\hline & PVBIPS & PDMS & $\mathrm{H}_{2} \mathrm{O}$ & $\mathrm{K}^{+}$ & $\mathrm{Na}^{+}$ & $>10$ & tribometer & $6 \mathrm{~mm}$ & brush & $48 \mathrm{~g}$ \\
\hline
\end{tabular}

* based on the given detection limit 


\subsubsection{Relation between friction and adhesion}

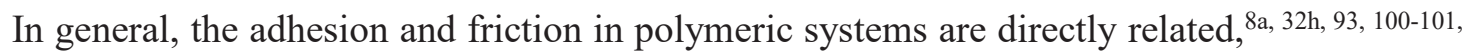
128 which indicates that they are caused by the same interactions. However, also some counterexamples have been reported. ${ }^{8 a}, 116$ For example, Song et al. ${ }^{32 \mathrm{~h}}$ fabricated PFDMS films on gold surface, and used AFM to study the COF and adherence strength at oxidized and reduced state of the polymers. After oxidization, both friction and adhesion switched to higher values. The process was found to be reversible: At the oxidized state, with varying electrolyte from $\mathrm{NaClO}_{4}$ to $\mathrm{NaNO}_{3}$, a decrease in both friction and adhesion was found. Thus, similar trends are found for switchable friction and adhesion is obtained through oxidization and varying electrolyte in these systems. On the other hand, Nordgren et l. $^{8 \mathrm{a}}$ grafted thermal and $\mathrm{pH}$ sensitive PDMAEMA brushes on a gold probe and gold-coated substrate. With this system, they switched adhesion and friction by varying both temperature and $\mathrm{pH}$ using water as a solvent. Above the LCST, lower friction is found with a relatively higher adhesion compared to below the LCST. Using the $\mathrm{pH}$ as the stimulus, the friction forces increased with increasing $\mathrm{pH}$. No systematic research was done on the effect of the $\mathrm{pH}$ on switchable adhesion. Nevertheless, at $\mathrm{pH}=11$, there is a clear temperature-dependent effect on the adhesion. While only repulsive forces between the opposing brushes were measured below the LCST, above the LCST, was found to be high, which is consistent with the trend of friction. Their results show us that under influence of different stimuli, friction and adhesion can show different relations. Using a $\mathrm{pH}$ stimulus, friction and adhesion are related, while for a thermal stimulus, they are not necessarily related.

\subsubsection{Relation between friction and adhesion hysteresis}

Next to the relation between adhesion and friction, also the relation between friction and adhesion hysteresis has been studied extensively. ${ }^{25}$, 120a, 120c, 129 Correlations between them have both been studied theoretically ${ }^{130}$ and experimentally. ${ }^{131}$ In general, when friction and adhesion hysteresis are caused by the same dissipation mechanism, they can be related. For the polymeric systems studied experimentally, this was often found to be the case. For example, Israelachvili et al. ${ }^{29 \mathrm{~d}}$ compared the friction-adhesion hysteresis relationship of surfactants in different phases (solid, amorphous, liquid). Their results revealed that adhesion hysteresis varies in exactly the same way as the friction force does. The same results also were obtained for fluorocarbon surfactant monolayer-coated surfaces. ${ }^{132}$ Generally, large friction forces are associated with large adhesion hysteresis. On the aspect of polymer films, also strong correlations are found between the friction and adhesion hysteresis. For example, Maeda et al. ${ }^{120 \mathrm{c}}$ coated PS and polyvinyl benzyl chloride (PVBC) on mica surface, and the adhesion and friction were studied using an SFA. After PVBC is crosslinked, the friction force is smaller than that without crosslinking. In comparison, chain scission of the outermost PS layers is achieved by UV irradiation, by which the 
friction forces and adhesion hysteresis increase significantly. The results show that friction is correlated with the adhesion hysteresis between two coated polymer surfaces. Moreover, Chaudhury et al. ${ }^{25}$ measured both friction and adhesion hysteresis of two asymmetric surfaces. One is poly(dimethylsiloxanes) (PDMS) surface, and the other is chemically modified mica surfaces. With varying the end group of modified monolayers, the adhesion hysteresis and friction are measured. The results show that friction and adhesion hysteresis have the same general trend. They conclude that adhesion hysteresis can be used to predict friction between two surfaces.

\subsection{Summary}

In this chapter, we presented an overview of the different stimulus responsive polymeric systems with which a switchable response can be enabled. Triggered by the surrounding environment, such as temperature, $\mathrm{pH}$, solvent type and mixture, redox, light, magnetic field, the chemical structure or composition of polymers change. Consequently, upon functionalizing surfaces with such polymers, the frictional response to sliding and adhesion can be tuned. The various stimulus methods and results give us a direct impression on how to control friction and adhesion effectively. Moreover, the relationship between friction and adhesion/adhesion hysteresis has also been discussed, which will offer researchers to explore more feasible and effective way to switch tribological properties on the surfaces. 


\section{Abbreviations}

PDEA: $\operatorname{poly}(N, N$-Diethylacrylamide $)$

PNIPAM: $\operatorname{poly}(N$-isopropylacrylamide)

PDMAEMA: poly[2-(dimethylamino)ethyl methacrylate]

PNIPAM- $b$-PAMPTMA: poly( $N$-isopropylacrylamide)-block-poly(3-acrylamidopropyl)trimethylammonium chloride

P(OEGMA-co-OPGMA): poly(oligo(ethylene glycol) methyl ether methacrylate-co-oligo(propylene glycol) methacrylate)

P(MEO2MA-co-OEGMA): poly(2-(2-methoxyethoxy) ethyl methacrylate-co-oligo(ethylene glycol) methyl ether methacrylate)

PS- $b$-PAA: polystyrene- $b$-poly(acrylic acid)

PMPC: poly(2-(methacryloyloxy)ethylphosphorylcholine)

PFS-I: poly(ferrocenyl(3-iodopropyl)methylsilane)

PFDMS: poly(ferrocenyl dimethylsilane)

PDMAEMA-stat-BPMA: poly(2-(dimethylamino)-ethyl methacrylate-stat-benzophenone methacrylate

PNIPAM-NaMA: poly( $N$-isopropylacrylamide)-poly(sodium methacrylate)

PMAA: poly(methacrylic acid)

PV2P: poly(2-vinylpyridine)

P(METAC)- $b$-(PEO $\left.{ }_{45} \mathrm{MEMA}\right)$ : poly(methacryloxyethyl) trimethylammonium chloride-block- poly-(ethylene oxide) methylether methacrylate

PSPMA: poly(3-sulfopropyl methacrylate potassium salt)

PVBIPS: poly(3-(1-(4-vinylbenzyl)-1 $H$-imidazol-3-ium-3-yl)propane-1-sulfonate)

PDMS: poly(dimethylsiloxane)

BSA: Bovine Serum Albumin

MUA: mercaptoundecanoic acid

GO: graphene oxide

CTAB: hexadecyltrimethylammonium bromide

SDS: sodium dodecyl sulfate 


\subsection{References}

[1] S. Milner, Science 1991, 251, 905-914.

[2] a) W. J. Brittain and S. Minko, Journal of Polymer Science Part a-Polymer Chemistry 2007, 45, 35053512; b) S. T. Milner, T. A. Witten and M. E. Cates, Macromolecules 1988, 21, 2610-2619; c) P. G. Degennes, Macromolecules 1980, 13, 1069-1075; d) P. G. Degennes, Advances in Colloid and Interface Science 1987, 27, 189-209.

[3] T. Wu, K. Efimenko and J. Genzer, Journal of the American Chemical Society 2002, 124, 9394-9395.

[4] R. Descas, J. U. Sommer and A. Blumen, Macromolecular Theory and Simulations 2008, 17, 429-453.

[5] R. A. Jones, Soft condensed matter, Oxford University Press, 2002, p.

[6] M. Kim, S. K. Schmitt, J. W. Choi, J. D. Krutty and P. Gopalan, Polymers 2015, 7, 1346-1378.

[7] S. Alexander, Journal De Physique 1977, 38, 983-987.

[8] a) N. Nordgren and M. W. Rutland, Nano Letters 2009, 9, 2984-2990; b) M. Stamm, Polymer Surfaces and Interfaces: Characterization, Modification and Applications, ISBN 978-3-540-73864-0. Springer Berlin Heidelberg, 2008 2008, 1.

[9] a) T. Chen, R. Ferris, J. M. Zhang, R. Ducker and S. Zauscher, Progress in Polymer Science 2010, 35, 94112; b) X. F. Sui, Q. Chen, M. A. Hempenius and G. J. Vancso, Small 2011, 7, 1440-1447.

[10] I. B. Malham and L. Bureau, Langmuir 2010, 26, 4762-4768.

[11] a) M. Chen, W. H. Briscoe, S. P. Armes and J. Klein, science 2009, 323, 1698-1701; b) Y. L. Yu, B. D. Kieviet, F. Liu, I. Siretanu, E. Kutnyanszky, G. J. Vancso and S. de Beer, Soft Matter 2015, 11, 8508-8516.

[12] a) K. Glinel, A. M. Jonas, T. Jouenne, J. Leprince, L. Galas and W. T. S. Huck, Bioconjugate Chemistry 2009, 20, 71-77; b) G. Z. Gao, K. Yu, J. Kindrachuk, D. E. Brooks, R. E. W. Hancock and J. N. Kizhakkedathu, Biomacromolecules 2011, 12, 3715-3727.

[13] a) S. Choi, B. C. Choi, C. Y. Xue and D. Leckband, Biomacromolecules 2013, 14, 92-100; b) S. Desseaux and H. A. Klok, Biomacromolecules 2014, 15, 3859-3865.

[14] a) I. Tokareva, S. Minko, J. H. Fendler and E. Hutter, Journal of the American Chemical Society 2004, 126, 15950-15951; b) H. M. Wang, L. X. Mu, L. L. Jin, G. W. She, H. T. Xu and W. S. Shi, Rsc Advances 2014, 4, 60086-60091.

[15] a) Z. Y. Bao, M. L. Bruening and G. L. Baker, Abstracts of Papers of the American Chemical Society 2005, 230, U4194-U4195; b) C. J. Kang, R. M. Crockett and N. D. Spencer, Macromolecules 2014, 47, 269275; c) S. H. Lee, D. R. Dreyer, J. H. An, A. Velamakanni, R. D. Piner, S. Park, Y. W. Zhu, S. O. Kim, C. W. Bielawski and R. S. Ruoff, Macromolecular Rapid Communications 2010, 31, 281-288; d) K. Matyjaszewski, Macromolecules 2012, 45, 4015-4039.

[16] R. Barbey, L. Lavanant, D. Paripovic, N. Schuwer, C. Sugnaux, S. Tugulu and H. A. Klok, Chemical Reviews 2009, 109, 5437-5527.

[17] S. Edmondson, V. L. Osborne and W. T. S. Huck, Chemical Society Reviews 2004, 33, 14-22.

[18] X. Y. Huang and M. J. Wirth, Analytical Chemistry 1997, 69, 4577-4580.

[19] M. Mozafari, V. Shabafrooz, M. Yazdimamaghani, D. Vashaee and L. Tayebi, Nanomedicine 489, 489.

[20] J. Pyun, T. Kowalewski and K. Matyjaszewski, Macromolecular Rapid Communications 2003, 24, 1043 1059.

[21] J. N. Israelachvili, Intermolecular and surface forces: revised third edition, Academic press, 2011, p.

[22] B. Bhushan, Introduction to tribology, John Wiley \& Sons, 2013, p.

[23] a) H. Zeng, Polymer adhesion, friction, and lubrication, John Wiley \& Sons, 2013, p; b) Z. Wei, M. F. He and Y. P. Zhao, Journal of Adhesion Science and Technology 2010, 24, 1045-1054.

[24] M. K. Chaudhury and G. M. Whitesides, Langmuir 1991, 7, 1013-1025.

[25] M. K. Chaudhury and M. J. Owen, Langmuir 1993, 9, 29-31.

[26] a) A. Stevenson in Vol. Oxford University Press, Oxford, UK, 2010; b) M. H. Muser, M. Urbakh and M. O. Robbins, Advances in Chemical Physics, Vol 126 2003, 126, 187-272.

[27] G. He, M. H. Muser and M. O. Robbins, Science 1999, 284, 1650-1652.

[28] L. Prandtl, Z Angew Math Mech 1928, 8, 85.

[29] a) J. Klein, Annual Review of Materials Science 1996, 26, 581-612; b) A. Galuschko, L. Spirin, T. Kreer, A. Johner, C. Pastorino, J. Wittmer and J. Baschnagel, Langmuir 2010, 26, 6418-6429; c) L. Spirin, A. Galuschko, T. Kreer, A. Johner, J. Baschnagel and K. Binder, European Physical Journal E 2010, 33, $307-$ 311; d) H. Yoshizawa, Y. L. Chen and J. Israelachvili, Journal of Physical Chemistry 1993, 97, 4128-4140.

[30] S. de Beer, Langmuir 2014.

[31] a) P. Attard, Journal of Adhesion Science and Technology 2002, 16, 753-791; b) M. C. Ben Jemaa, R. Mnif, K. Fehri and R. Elleuch, Tribology Letters 2012, 45, 177-184; c) M. A. Cole, N. H. Voelcker, H. Thissen, R. G. Horn and H. J. Griesser, Soft Matter 2010, 6, 2657-2667; d) P. R. De Silva, M. Priest, P. M. Lee, R. C. Coy and R. I. Taylor, Tribology Letters 2011, 43, 107-120; e) D. Pavkovic, N. Kranjcevic and M. 
Kostelac, Automatika 2013, 54, 364-375; f) H. Vink, European Polymer Journal 1971, 7, 1411-\&; g) H. B. Yu, G. Y. Zhou, X. Chew, S. K. Sinha and F. S. Chau, Journal of Micromechanics and Microengineering 2013, 23; h) M. Benz, T. Gutsmann, N. H. Chen, R. Tadmor and J. Israelachvili, Biophysical Journal 2004, 86, 870-879; i) M. Ruths, N. Alcantar and J. Israelachvili, Abstracts of Papers of the American Chemical Society 2003, 225, U616-U616; j) H. Zeng, Y. Tian, T. H. Anderson, M. Tirrell and J. N. Israelachvili, Langmuir 2008, 24, 1173-1182.

[32] a) S. de Beer, Langmuir 2014, 30, 8085-8090; b) S. de Beer, E. Kutnyanszky, M. H. Muser and G. J. Vancso, Jove-Journal of Visualized Experiments 2014, 52285; c) S. de Beer and M. H. Muser, Soft Matter 2013, 9, 7234-7241; d) S. de Beer and M. H. Muser, Macromolecules 2014, 47, 7666-7673; e) Y. Yu, B. D. Kieviet, F. Liu, I. Siretanu, E. Kutnyanszky, G. J. Vancso and S. de Beer, Soft Matter 2015, 11, 8508-8516; f) Y. L. Yu, B. D. Kieviet, E. Kutnyanszky, G. J. Vancso and S. de Beer, Acs Macro Letters 2015, 4, 75-79; g) Q. Chen, E. S. Kooij, X. F. Sui, C. J. Padberg, M. A. Hempenius, P. M. Schon and G. J. Vancso, Soft Matter 2014, 10, 3134-3142; h) J. Song and G. J. Vancso, Langmuir 2011, 27, 6822-6829.

[33] a) J. N. Israelachvili, Y. L. Chen and H. Yoshizawa, Journal of Adhesion Science and Technology 1994, 8, 1231-1249; b) J. Klein, E. Kumacheva, D. Mahalu, D. Perahia and L. J. Fetters, Nature 1994, 370, 634-636; c) J. Klein, E. Kumacheva, D. Perahia and L. J. Fetters, Acta Polymerica 1998, 49, 617-625.

[34] a) Q. B. Wei, M. R. Cai, F. Zhou and W. M. Liu, Macromolecules 2013, 46, 9368-9379; b) C. W. Wu, G. J. Ma, P. Zhou and C. D. Wu, Journal of Tribology-Transactions of the Asme 2006, 128, 904-907; c) Y. Wu, M. R. Cai, X. W. Pei, Y. M. Liang and F. Zhou, Macromolecular Rapid Communications 2013, 34, 17851790; d) Y. Wu, Q. B. Wei, M. R. Cai and F. Zhou, Advanced Materials Interfaces 2015, 2.

[35] T. Ushiki, J. Hitomi, S. Ogura, T. Umemoto and M. Shigeno, Archives of Histology and Cytology 1996, $59,421-431$.

[36] G. Binnig, C. F. Quate and C. Gerber, Physical Review Letters 1986, 56, 930-933.

[37] H. Schonherr and G. J. Vancso, Scanning Force Microscopy of Polymers, Springer, Verlag Berlin Heidelberg, 2010, p.

[38] K. Wagner, P. Cheng and D. Vezenov, Langmuir 2011, 27, 4635-4644.

[39] a) A. Janshoff, M. Neitzert, Y. Oberdorfer and H. Fuchs, Angewandte Chemie-International Edition 2000, 39, 3213-3237; b) Y. K. Lai, Y. X. Tang, J. Y. Huang, F. Pan, Z. Chen, K. Q. Zhang, H. Fuchs and L. F. Chi, Scientific Reports 2013, 3.

[40] a) D. Dietzel, C. Ritter, T. Monninghoff, H. Fuchs, A. Schirmeisen and U. D. Schwarz, Physical Review Letters 2008, 101; b) A. Schirmeisen, L. Jansen, H. Holscher and H. Fuchs, Applied Physics Letters 2006, 88.

[41] a) M. E. Dokukin and I. Sokolov, Langmuir 2012, 28, 16060-16071; b) J. Duvigneau, H. Schonherr and G. J. Vancso, Acs Nano 2010, 4, 6932-6940; c) R. Hiesgen, I. Wehl, S. Helmly, A. Haug, M. Schulze, A. Bauder, H. Wang, X.-Z. Yuan and K. Andreas Friedrich, Journal of Electroanalytical Chemistry 2011, 662, 240-250; d) B. Pittenger, N. Erina and C. Su, Application Note Veeco Instruments Inc 2010; e) H. Schönherr and G. J. Vancso in Physical Principles of Scanning Probe Microscopy Imaging, Springer, 2010, pp. 3-24; f) G. J. Vancso and H. Schönherr, Scanning force microscopy of polymers, Springer, 2010, p.

[42] a) H. Fischer, H. Stadler and N. Erina, Journal of microscopy 2013, 250, 210-217; b) H. Giray Oral, Z. Parlak and F. Levent Degertekin, Ultramicroscopy 2012, 120, 56-63; c) A. Oláh, H. Hillborg and G. J. Vancso, Applied Surface Science 2005, 239, 410-423; d) W. Walczyk, P. M. Schön and H. Schönherr, Journal of Physics: Condensed Matter 2013, 25, 184005; e) X. Yu, N. A. Burnham, R. B. Mallick and M. Tao, Fuel 2013, 113, 443-447.

[43] a) F. Benmouna and D. Johannsmann, Langmuir 2004, 20, 188-193; b) M. Gelbert, M. Biesalski, J. Rühe and D. Johannsmann, Langmuir 2000, 16, 5774-5784; c) A. Roters, M. Gelbert, M. Schimmel, J. Rühe and D. Johannsmann, Physical Review E 1997, 56, 3256; d) A. Roters and D. Johannsmann, Journal of Physics: Condensed Matter 1996, 8, 7561; e) A. Roters, M. Schimmel, J. Rühe and D. Johannsmann, Langmuir 1998, 14, 3999-4004.

[44] a) M. P. De Boer, J. A. Knapp, T. A. Michalske, U. Srinivasan and R. Maboudian, Acta Materialia 2000, 48, 4531-4541; b) B. Gotsmann, C. Seidel, B. Anczykowski and H. Fuchs, Physical Review B 1999, 60, 11051-11061.

[45] a) G. Bogdanovic, A. Meurk and M. W. Rutland, Colloids and Surfaces B-Biointerfaces 2000, 19, 397405; b) A. Feiler, P. Attard and I. Larson, Review of Scientific Instruments 2000, 71, 2746-2750.

[46] a) C. Wu and X. H. Wang, Physical Review Letters 1998, 80, 4092-4094; b) T. Tanaka, Physical Review Letters 1978, 40, 820-823; c) E. M. Benetti, S. Zapotoczny and J. Vancso, Advanced Materials 2007, 19, 268271; d) X. F. Sui, A. Di Luca, M. K. Gunnewiek, E. S. Kooij, C. A. van Blitterswijk, L. Moroni, M. A. Hempenius and G. J. Vancso, Australian Journal of Chemistry 2011, 64, 1259-1266; e) X. F. Sui, S. Zapotoczny, E. M. Benetti, M. Memesa, M. A. Hempenius and G. J. Vancso, Polymer Chemistry 2011, 2, 879-884. 
[47] a) S. S. Shiratori and M. F. Rubner, Macromolecules 2000, 33, 4213-4219; b) T. Tanaka, D. Fillmore, S.T. Sun, I. Nishio, G. Swislow and A. Shah, Physical Review Letters 1980, 45, 1636; c) D. T. Auguste, S. P. Armes, K. R. Brzezinska, T. J. Deming, J. Kohn and R. K. Prud'homme, Biomaterials 2006, 27, 2599-2608; d) J. Z. Du, Y. Q. Tang, A. L. Lewis and S. P. Armes, Journal of the American Chemical Society 2005, 127, 17982-17983; e) A. M. El Badawy, T. P. Luxton, R. G. Silva, K. G. Scheckel, M. T. Suidan and T. M. Tolaymat, Environmental Science \& Technology 2010, 44, 1260-1266; f) H. K. Ju, S. Y. Kim and Y. M. Lee, Polymer 2001, 42, 6851-6857; g) D. Schmaljohann, Advanced Drug Delivery Reviews 2006, 58, 1655-1670; h) P. S. Uster and D. W. Deamer, Biochemistry 1985, 24, 1-8; i) Y. Xu, S. Bolisetty, M. Drechsler, B. Fang, J. Yuan, M. Ballauff and A. H. Müller, Polymer 2008, 49, 3957-3964.

[48] a) S. Ghosh, V. Yesilyurt, E. N. Savariar, K. Irvin and S. Thayumanavan, Journal of Polymer Science Part a-Polymer Chemistry 2009, 47, 1052-1060; b) I. Ohmine and T. Tanaka, Journal of Chemical Physics 1982, 77, 5725-5729; c) Y. K. Jhon, R. R. Bhat, C. Jeong, O. J. Rojas, I. Szleifer and J. Genzer, Macromolecular rapid communications 2006, 27, 697-701; d) B. Lego, W. G. Skene and S. Giasson, Macromolecules 2010, 43, 4384-4393; e) J. Mahon and S. Zhu, Colloid and Polymer Science 2008, 286, 1443-1454; f) H. Matsuoka, Y. Yamakawa, A. Ghosh and Y. Saruwatari, Langmuir 2015, 31, 4827-4836; g) J. T. Yang, H. Chen, S. W. Xiao, M. X. Shen, F. Chen, P. Fan, M. Q. Zhong and J. Zheng, Langmuir 2015, 31, 9125-9133; h) Z. Y. Zhang, M. Moxey, A. Alswieleh, A. J. Morse, A. L. Lewis, M. Geoghegan and G. J. Leggett, Langmuir 2016, 32, 5048-5057.

[49] a) Z. Y. Zhang, A. J. Morse, S. P. Armes, A. L. Lewis, M. Geoghegan and G. J. Leggett, Langmuir 2011, 27, 2514-2521; b) F. M. Winnik, H. Ringsdorf and J. Venzmer, Macromolecules 1990, 23, 2415-2416.

[50] a) B. I. Ipe, S. Mahima and K. G. Thomas, Journal of the American Chemical Society 2003, 125, $7174-$ 7175; b) M. Irie and D. Kungwatchakun, Makromolekulare Chemie-Rapid Communications 1984, 5, 829-832; c) D. Q. Liu and D. J. Broer, Angewandte Chemie-International Edition 2014, 53, 4542-4546; d) D. Q. Liu and D. J. Broer, Soft Matter 2014, 10, 7952-7958.

[51] a) X. Feng, X. Sui, M. A. Hempenius and G. J. Vancso, Journal of the American Chemical Society 2014, 136, 7865-7868; b) X. Sui, X. Feng, J. Song, M. A. Hempenius and G. J. Vancso, Journal of materials chemistry 2012, 22, 11261-11267; c) J. Song, M. A. Hempenius, H. J. Chung and G. J. Vancso, Nanoscale 2015, 7, 9970-9974; d) J. Vancso, X. F. Sui, L. L. Shui, A. van den Berg and M. Hempenius, Abstracts of Papers of the American Chemical Society 2011, 242; e) K. Zhang, X. Feng, X. Sui, M. A. Hempenius and G. J. Vancso, Angewandte Chemie 2014, 126, 14009-14013; f) B. Zoetebier, M. A. Hempenius and G. J. Vancso, Chemical Communications 2015, 51, 636-639; g) B. Zoetebier, A. Sohrabi, B. Lou, M. A. Hempenius, W. E. Hennink and G. J. Vancso, Chemical Communications 2016, 52, 7707-7710; h) B. Zoetebier, S. Tas, G. J. Vancso, K. Nijmeijer and M. A. Hempenius, Journal of Polymer Science Part a-Polymer Chemistry 2015, 53, 2786-2793.

[52] a) M. A. C. Stuart, W. T. S. Huck, J. Genzer, M. Muller, C. Ober, M. Stamm, G. B. Sukhorukov, I. Szleifer, V. V. Tsukruk, M. Urban, F. Winnik, S. Zauscher, I. Luzinov and S. Minko, Nature Materials 2010, 9, 101-113; b) T. Tanaka, I. Nishio, S. T. Sun and S. Uenonishio, Science 1982, 218, 467-469.

[53] V. Bergeron, D. Bonn, J. Y. Martin and L. Vovelle, Nature 2000, 405, 772-775.

[54] Y. Wu, Y. H. Xue, X. W. Pei, M. R. Cai, H. L. Duan, W. T. S. Huck, F. Zhou and Q. J. Xue, Journal of Physical Chemistry C 2014, 118, 2564-2569.

[55] a) D. P. Chang, J. E. Dolbow and S. Zauscher, Langmuir 2007, 23, 250-257; b) J.-K. Chen, J.-H. Wang, J.-Y. Chang and S.-K. Fan, Applied Physics Letters 2012, 101, 123701; c) J. Elbert, M. Gallei, C. Rüttiger, A. Brunsen, H. Didzoleit, B. Stühn and M. Rehahn, Organometallics 2013, 32, 5873-5878; d) M. Ilcikova, J. Tkac and P. Kasak, Polymers 2015, 7, 2344-2370; e) R. Jakubiak, T. J. Bunning, R. A. Vaia, L. V. Natarajan and V. P. Tondiglia, Advanced Materials 2003, 15, 241-244; f) M. Motornov, T. K. Tam, M. Pita, I. Tokarev, E. Katz and S. Minko, Nanotechnology 2009, 20, 434006; g) S. Schmidt, M. Zeiser, T. Hellweg, C. Duschl, A. Fery and H. Mohwald, Advanced Functional Materials 2010, 20, 3235-3243; h) E. Svetushkina, N. Puretskiy, L. Ionov, M. Stamm and A. Synytska, Soft Matter 2011, 7, 5691-5696.

[56] U. Gedde, Polymer physics, Springer Science \& Business Media, 1995, p.

[57] I. C. Sanchez and R. H. Lacombe, Macromolecules 1978, 11, 1145-1156.

[58] C. Panayiotou and I. C. Sanchez, Macromolecules 1991, 24, 6231-6237.

[59] a) G. M. Kontogeorgis, A. Saraiva, A. Fredenslund and D. P. Tassios, Industrial \& Engineering Chemistry Research 1995, 34, 1823-1834; b) J. M. Rodriguezparada and V. Percec, Journal of Polymer Science Part a-Polymer Chemistry 1986, 24, 579-589.

[60] a) R. Liu, M. Fraylich and B. R. Saunders, Colloid and Polymer Science 2009, 287, 627-643; b) T. G. McKenzie, E. H. Wong, Q. Fu, S. J. Lam, D. E. Dunstan and G. G. Qiao, Macromolecules 2014, 47, 78697877; c) M. Heskins and J. E. Guillet, Journal of Macromolecular Science: Part A - Chemistry 1968, 2, 14411455 . 
[61] a) G. Dalkas, K. Pagonis and G. Bokias, Polymer 2006, 47, 243-248; b) D. Mukherji, C. M. Marques, T. Stuehn and K. Kremer, The Journal of chemical physics 2015, 142, 114903; c) C. Scherzinger, P. Lindner, M. Keerl and W. Richtering, Macromolecules 2010, 43, 6829-6833; d) C. Scherzinger, A. Schwarz, A. Bardow, K. Leonhard and W. Richtering, Current Opinion in Colloid \& Interface Science 2014, 19, 84-94; e) F. M. Winnik, M. F. Ottaviani, S. H. Bossmann, M. Garcia-Garibay and N. J. Turro, Macromolecules 1992, 25, 6007-6017.

[62] a) R. Hoogenboom, C. R. Becer, C. Guerrero-Sanchez, S. Hoeppener and U. S. Schubert, Australian journal of chemistry 2010, 63, 1173-1178; b) S. M. Lee and Y. C. Bae, Polymer 2014, 55, 4684-4692; c) D. Mukherji and K. Kremer, Macromolecules 2013, 46, 9158-9163; d) S. Y. Oh and Y. C. Bae, Polymer 2013, 54, 2308-2314; e) B. R. Saunders, H. M. Crowther and B. Vincent, Macromolecules 1997, 30, 482-487; f) T. H. Young and W. Y. Chuang, Journal of Membrane Science 2002, 210, 349-359; g) G. Z. Zhang and C. Wu, Physical Review Letters 2001, 86, 822-825; h) P. W. Zhu and D. H. Napper, Journal of Colloid and Interface Science 1996, 177, 343-352.

[63] R. L. Scott, The Journal of Chemical Physics 1949, 17, 268-279.

[64] F. Tanaka, T. Koga and F. M. Winnik, Physical review letters 2008, 101, 028302.

[65] M. Z. Liu, F. L. Bian and F. L. Sheng, European Polymer Journal 2005, 41, 283-291.

[66] F. Rodriguez-Ropero, T. Hajari and N. F. A. van der Vegt, Journal of Physical Chemistry B 2015, 119, $15780-15788$.

[67] a) F. M. Winnik, H. Ringsdorf and J. Venzmer, Macromolecules 1990, 23, 2415-2416; b) B. L. Liu, J. Wang, G. Y. Ru, C. Y. Liu and J. W. Feng, Macromolecules 2015, 48, 1126-1133.

[68] a) F. M. Winnik, M. F. Ottaviani, S. H. Bossmann, W. Pan, M. Garcia-Garibay and N. J. Turro, Macromolecules 1993, 26, 4577-4585; b) C. W. Tu and S. W. Kuo, Journal of Polymer Research $2014,21$.

[69] P. W. Zhu and D. H. Napper, Chemical physics letters 1996, 256, 51-56.

[70] H. Yamauchi and Y. Maeda, The Journal of Physical Chemistry B 2007, 111, 12964-12968.

[71] L. Liu, X. L. Song, X. J. Ju, R. Xie, Z. Liu and L. Y. Chu, Journal of Physical Chemistry B 2012, 116 , 974-979.

[72] Y. Ito, T. Ito, H. Takaba and S. Nakao, Journal of Membrane Science 2005, 261, 145-151.

[73] H. J. Dai, Q. Chen, H. L. Qin, Y. Guan, D. Y. Shen, Y. Q. Hua, Y. L. Tang and J. Xu, Macromolecules 2006, 39, 6584-6589.

[74] a) M. Muller, T. Rieser, P. L. Dubin and K. Lunkwitz, Macromolecular Rapid Communications 2001, 22, 390-395; b) M. Mostafavi, N. Keghouche and M.-O. Delcourt, Chemical Physics Letters 1990, 169, 81-84; c) K. C. Clarke, S. N. Dunham and L. A. Lyon, Chemistry of Materials 2015, 27, 1391-1396.

[75] a) Y. Ding, X. H. Xia and H. S. Zhai, Chemistry-a European Journal 2007, 13, 4197-4202; b) C. Leng, X. F. Han, Q. Shao, Y. H. Zhu, Y. T. Li, S. Y. Jiang and Z. Chen, Journal of Physical Chemistry C 2014, 118, 15840-15845; c) J. W. Lu, H. Y. Jia, L. J. Guo, G. H. Zhang, Y. J. Cao, H. S. Yan and K. L. Liu, European Polymer Journal 2015, 66, 376-385.

[76] a) E. V. Korchagina and O. E. Philippova, Macromolecules 2015, 48, 8622-8628; b) K. Ogawa, Advances in colloid and interface science 2015, 226, 115-121; c) W. Wang, Y. Xu, S. Backes, A. Li, S. Micciulla, A. B. Kayitmazer, L. Li, X. Guo and R. von Klitzing, Langmuir 2016.

[77] a) L. Ni, J. Meng, G. M. Geise, Y. Zhang and J. Zhou, Journal of Membrane Science 2015, 491, 73-81; b) T. Xiang, C.-D. Luo, R. Wang, Z.-Y. Han, S.-D. Sun and C.-S. Zhao, Journal of Membrane Science 2015, 476, 234-242; c) T. Wang, R. Kou, H. Liu, L. Liu, G. Zhang and G. Liu, Langmuir 2016, 32, 2698-2707.

[78] a) M. Irie, Y. Hirano, S. Hashimoto and K. Hayashi, Macromolecules 1981, 14, 262-267; b) Y. Zhao and T. Ikeda, Smart light-responsive materials: azobenzene-containing polymers and liquid crystals, John Wiley \& Sons, 2009, p; c) J.-F. Gohy and Y. Zhao, Chemical Society Reviews 2013, 42, 7117-7129.

[79] N. K. Mal, M. Fujiwara and Y. Tanaka, Nature 2003, 421, 350-353.

[80] F. Ercole, T. P. Davis and R. A. Evans, Polymer Chemistry 2010, 1, 37-54.

[81] J. Liu, J. L. Yan, X. W. Yuan, K. Q. Liu, J. X. Peng and Y. Fang, Journal of Colloid and Interface Science 2008, 318, 397-404.

[82] Y. L. Zhao, I. Aprahamian, A. Trabolsi, N. Erina and J. F. Stoddart, Journal of the American Chemical Society 2008, 130, 6348-+.

[83] D. J. Irvin, S. H. Goods and L. L. Whinnery, Chemistry of Materials 2001, 13, 1143-1145.

[84] F. Peng, G. Z. Li, X. X. Liu, S. Z. Wu and Z. Tong, Journal of the American Chemical Society 2008, 130, $16166-+$.

[85] N. V. Tsarevsky and K. Matyjaszewski, Macromolecules 2005, 38, 3087-3092.

[86] a) M. Kesik, H. Akbulut, S. Söylemez, Ş. C. Cevher, G. Hızalan, Y. A. Udum, T. Endo, S. Yamada, A. Çırpan and Y. Yağc1, Polymer Chemistry 2014, 5, 6295-6306; b) L. C. Liu, L. L. Rui, Y. Gao and W. A. Zhang, Polymer Chemistry 2015, 6, 1817-1829.

[87] L. Dos Ramos, S. de Beer, M. A. Hempenius and G. J. Vancso, Langmuir 2015, 31, 6343-6350. 
[88] a) R. Cheng, F. Meng, C. Deng, H.-A. Klok and Z. Zhong, Biomaterials 2013, 34, 3647-3657; b) J. Zhuang, M. R. Gordon, J. Ventura, L. Li and S. Thayumanavan, Chemical Society Reviews 2013, 42, 74217435; c) Y. Dong, J. Zhang, X. Tan, L. Wang, J. Chen, B. Li, L. Ye, B. Xu, B. Zou and W. Tian, Journal of Materials Chemistry C 2013, 1, 7554-7559.

[89] Y. Wu, M. Cai, X. Pei, Y. Liang and F. Zhou, Macromolecular rapid communications 2013, 34, 17851790.

[90] a) F. Costantini, E. M. Benetti, D. N. Reinhoudt, J. Huskens, G. J. Vancso and W. Verboom, Lab on a Chip 2010, 10, 3407-3412; b) S. de Beer, E. Kutnyanszky, P. M. Schon, G. J. Vancso and M. H. Muser, Nature Communications 2014, 5, 3781; c) D. Jańczewski, N. Tomczak, J. Song, H. Long, M.-Y. Han and G. J. Vancso, Journal of Materials Chemistry 2011, 21, 6487-6490; d) B. D. Kieviet, P. M. Schön and G. J. Vancso, Lab on a Chip 2014, 14, 4159-4170; e) H. Schönherr, M. W. Beulen, J. Bügler, J. Huskens, F. C. van Veggel, D. N. Reinhoudt and G. J. Vancso, Journal of the American Chemical Society 2000, 122, 4963-4967; f) O. Tagit, N. Tomczak, E. M. Benetti, Y. Cesa, C. Blum, V. Subramaniam, J. L. Herek and G. J. Vancso, Nanotechnology 2009, 20; g) O. Tagit, N. Tomczak and G. J. Vancso, Small 2008, 4, 119-126; h) E. Tocha, H. Schönherr and G. J. Vancso, Soft Matter 2009, 5, 1489-1495.

[91] S. T. Milner, Science 1991, 251, 905-914.

[92] D. M. Jones, J. R. Smith, W. T. S. Huck and C. Alexander, Advanced Materials 2002, 14, 1130-1134.

[93] X. Banquy, E. Charrault and S. Giasson, Journal of Physical Chemistry B 2010, 114, 9721-9728.

[94] D. M. Jones, J. R. Smith, W. T. Huck and C. Alexander, Advanced Materials 2002, 14, 1130.

[95] E. C. Cho, Y. D. Kim and K. Cho, Polymer 2004, 45, 3195-3204.

[96] A. Dedinaite, E. Thormann, G. Olanya, P. M. Claesson, B. Nystrom, A. L. Kjoniksen and K. Z. Zhu, Soft Matter 2010, 6, 2489-2498.

[97] A. Synytska, E. Svetushkina, N. Puretskiy, G. Stoychev, S. Berger, L. Ionov, C. Bellmann, K. J. Eichhorn and M. Stamm, Soft Matter 2010, 6, 5907-5914.

[98] S. Kessel, S. Schmidt, R. Muller, E. Wischerhoff, A. Laschewsky, J. F. Lutz, K. Uhlig, A. Lankenau, C. Duschl and A. Fery, Langmuir 2010, 26, 3462-3467.

[99] O. Hoy, B. Zdyrko, R. Lupitskyy, R. Sheparovych, D. Aulich, J. F. Wang, E. Bittrich, K. J. Eichhorn, P. Uhlmann, K. Hinrichs, M. Muller, M. Stamm, S. Minko and I. Luzinov, Advanced Functional Materials 2010, 20, 2240-2247.

[100] M. Raftari, Z. Y. Zhang, S. R. Carter, G. J. Leggett and M. Geoghegan, Soft Matter 2014, 10, 27592766 .

[101] M. K. Vyas, K. Schneider, B. Nandan and M. Stamm, Soft Matter 2008, 4, 1024-1032.

[102] X. F. Sui, X. L. Feng, J. Song, M. A. Hempenius and G. J. Vancso, Journal of Materials Chemistry 2012, 22, 11261-11267.

[103] R. S. Hoy and G. S. Grest, Macromolecules 2007, 40, 8389-8395.

[104] R. Tadmor, J. Janik, J. Klein and L. J. Fetters, Physical Review Letters 2003, 91.

[105] C. Pastorino, T. Kreer, M. Muller and K. Binder, Physical Review E 2007, 76.

[106] L. Ashton, P. Pudney, E. Blanch and G. Yakubov, Advances in colloid and interface science 2013, 199 , 66-77.

[107] T. Vilmin and E. Raphael, Journal of Adhesion 2006, 82, 517-526.

[108] X. Banquy, E. Charrault and S. Giasson, The Journal of Physical Chemistry B 2010, 114, 9721-9728.

[109] T. Tateishi, M. Kyomoto, S. Kakinoki, T. Yamaoka and K. Ishihara, Journal of Biomedical Materials Research Part A 2014, 102, 1342-1349.

[110] G. H. Hsiue, S. D. Lee, P. C. T. Chang and C. Y. Kao, Journal of Biomedical Materials Research 1998, 42, 134-147.

[111] G. R. Whittell, M. D. Hager, U. S. Schubert and I. Manners, Nature Materials 2011, 10, 176-188.

[112] X. Feng, B. D. Kieviet, J. Song, P. M. Schön and G. J. Vancso, Applied surface science 2014, 292, $107-$ 110.

[113] a) J. P. Gong, T. Kurokawa, T. Narita, G. Kagata, Y. Osada, G. Nishimura and M. Kinjo, Journal of the American Chemical Society 2001, 123, 5582-5583; b) S. H. Ma, D. A. Wang, Y. M. Liang, B. Q. Sun, S. N. Gorb and F. Zhou, Small 2015, 11, 1131-1137.

[114] M. H. Muser, Physical Review B 2011, 84.

[115] M. Kobayashi, Y. Terayama, N. Hosaka, M. Kaido, A. Suzuki, N. Yamada, N. Torikai, K. Ishihara and A. Takahara, Soft Matter 2007, 3, 740-746.

[116] J. Huang, B. Cusick, J. Pietrasik, L. Wang, T. Kowalewski, Q. Lin and K. Matyjaszewski, Langmuir 2007, 23, 241-249.

[117] Y. Wu, X. W. Pei, X. L. Wang, Y. M. Liang, W. M. Liu and F. Zhou, Npg Asia Materials 2014, 6. [118] X. Y. Liu, E. Thormann, A. Dedinaite, M. Rutland, C. Visnevskij, R. Makuska and P. M. Claesson, Soft Matter 2013, 9, 5361-5371. 
[119] R. Zhang, S. H. Ma, Q. B. Wei, Q. Ye, B. Yu, J. van der Gucht and F. Zhou, Macromolecules 2015, 48, 6186-6196.

[120] a) H. Yoshizawa, Y. L. Chen and J. Israelachvili, The Journal of Physical Chemistry 1993, 97, 41284140; b) H. Yoshizawa and J. Israelachvili, Journal of Physical Chemistry 1993, 97, 11300-11313; c) N. Maeda, N. H. Chen, M. Tirrell and J. N. Israelachvili, Science 2002, 297, 379-382.

[121] J. Ringlein and M. O. Robbins, American Journal of Physics 2004, 72, 884-891.

[122] M. H. Müser, L. Wenning and M. O. Robbins, Physical review letters 2001, 86, 1295.

[123] J. S. Mcfarlane and D. Tabor, Proceedings of the Royal Society of London Series a-Mathematical and Physical Sciences 1950, 202, 244-253.

[124] K. Autumn, A. Dittmore, D. Santos, M. Spenko and M. Cutkosky, Journal of Experimental Biology 2006, 209, 3569-3579.

[125] N. H. Chen, N. Maeda, M. Tirrell and J. Israelachvili, Macromolecules 2005, 38, 3491-3503.

[126] a) A. M. Homola, J. N. Israelachvili, M. L. Gee and P. M. Mcguiggan, Journal of TribologyTransactions of the Asme 1989, 111, 675-682; b) A. M. Homola, J. N. Israelachvili, P. M. Mcguiggan and M. L. Gee, Wear 1990, 136, 65-83; c) E. Rabinowicz, Friction and Wear of Materials, John Wiley, New York and London, 1965, p; d) E. D. Brown, R. S. Owens and E. R. Booser, Boundary Lubrication, ASME Publication, New York, 1969, p.

[127] M. Schindler, Chemical Physics 2010, 375, 327-336.

[128] A. Li, S. N. Ramakrishna, E. S. Kooij, R. M. Espinosa-Marzal and N. D. Spencer, Soft Matter 2012, 8, 9092-9100.

[129] B. Briscoe, D. Evans and D. Tabor, Journal of Colloid and Interface Science 1977, 61, 9-13.

[130] S. de Beer and M. H. Müser, Soft Matter 2013, 9, 7234-7241.

[131] R. Szoszkiewicz, B. Bhushan, B. D. Huey, A. J. Kulik and G. Gremaud, Journal of Chemical Physics 2005, 122 .

[132] S. Yamada and J. Israelachvili, Journal of Physical Chemistry B 1998, 102, 234-244 



\section{Chapter 3}

\section{Stretching of Collapsed Polymers Causes an Enhanced Dissipative Response of PNIPAM Brushes near their LCST *}

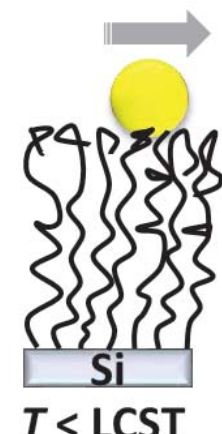

$T<$ LCST

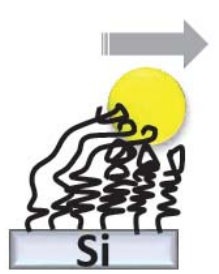

LCST
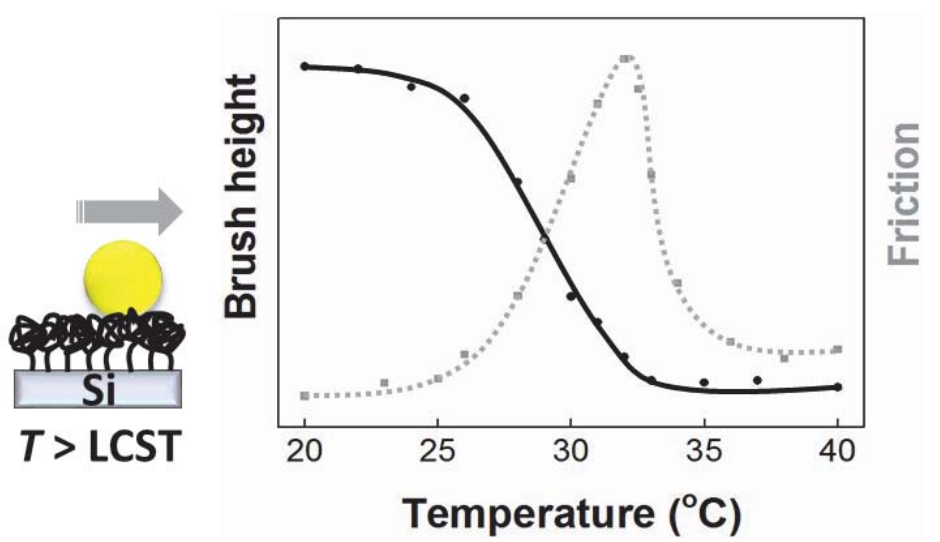

Poly( $N$-isopropyl acrylamide) (PNIPAM) is a stimulus-responsive polymer that can switch in water from an expanded state below the lower critical solution temperature (LCST) of $32{ }^{\circ} \mathrm{C}$ to a globular state above the LCST. It was recently shown, that, as a consequence of this conformational transition, the interfacial and (tribo-)mechanical properties of polymeric systems composed of PNIPAM can be switched between two states. Here we show that the tribo-mechanical properties of a particular type of PNIPAM systems, which is the PNIPAM brush, do not just change between two states, but instead evolve continuously and non-monotonically upon increasing/decreasing temperature. To do so, we present atomic force microscopy experiments in which we measure the adhesion hysteresis and the friction upon bringing a gold colloid in relative motion with PNIPAM brushes at temperatures around the LCST. Both the friction and the adhesion hysteresis display a pronounced maximum exactly at the LCST. The force $v s$ distance data captured at these temperatures shows a long-ranged adhesive interaction upon moving the colloid away from the original point of contact, which indicates that during this retraction the partly collapsed polymers in the brush become strongly stretched.

* This chapter has been published in: Yu, Y.; Kieviet, B. D.; Liu, F.; Siretanu, I.; Kutnyanszky, E.; Vancso, G. J.; de Beer, S., Soft Matter, 2015, 11, 8508-8516. 


\subsection{Introduction}

Polymers have been a popular ingredient of "smart", functional systems ${ }^{1}$ and as such have become enabling ingredients in nanosensors, ${ }^{2}$ switchable adhesives ${ }^{3}$ and (nano-)actuators. ${ }^{4}$ The mechanical and interfacial properties of these systems dramatically change in response to a small stimulus or modification of the environment. Stimuli that trigger these changes can be for example the oxidation state, ${ }^{5} \mathrm{pH},{ }^{6} \mathrm{UV}$ light radiation. ${ }^{7}$

For many applications, e.g. in drug delivery, ${ }^{8}$ for temperature-sensing ${ }^{9}$ or for bioadhesion, ${ }^{10}$ the temperature is used as a stimulus inducing a response of the polymeric system. In polymer-solvent mixtures, the conformational state of the polymers can be switched using either the upper critical solution temperature (UCST) or the lower critical solution temperature (LCST), provided that the critical temperature lies between the melting and the boiling point of the solvent. For an UCST, the solute dissolves in the solvent above the critical temperature, while for a LCST, a miscible solution is obtained below the critical temperature. Both UCST and LCST behavior are observed in a wide variety of polymer-solvent combinations. An example is poly[2-(methacryloyloxy)ethyl]dimethyl(3-sulfopropyl) ammonium hydroxide (PMEDSAH), ${ }^{11}$ which exhibits an UCST around $30{ }^{\circ} \mathrm{C}$ in aqueous solution. Also for polymers showing LCST behavior, there are many examples, such as poly(2-dimethylamino)ethyl methacrylate (PDMAEMA), ${ }^{12}$ polyethylene glycol (PEG), ${ }^{13}$ poly(di(ethylene glycol)methyl ether methacrylate) (PMEO $\left.{ }_{2} \mathrm{MA}\right){ }^{14}$ An especially well-studied polymer is poly( $N$-isopropyl acrylamide) (PNIPAM), ${ }^{15}$ which exhibits an LCST close to room temperature (typically $30-33{ }^{\circ} \mathrm{C}$ ) ${ }^{16}$ in pure water. ${ }^{17}$

When polymers are attached by one end to a surface or interface at a sufficiently high grafting density, the macromolecules stretch in the direction perpendicular to the grafting plane forming a so-called polymer brush. ${ }^{18}$ The degree of stretching of the polymers in brushes is determined by the solvent quality: in good solvents, the polymers stretch out and the solvent is absorbed in the brush, while the polymers form a dense film on the surface in poor solvents. Swollen brushes have been found to act as excellent lubricants, ${ }^{19}$ antibacterial or antifouling surfaces, ${ }^{20}$ cell adhesives ${ }^{21}$ and sensors. ${ }^{22}$ These properties can be made switchable when external stimuli are employed to change the effective solvent quality. $3 \mathrm{~b}, 21,23$

PNIPAM brushes are a convenient model-system to investigate the switchable interfacial properties of responsive surface-grafts, because PNIPAM has its LCST just above room temperature. Neutron reflectivity was used to confirm the difference in conformational state of the PNIPAM polymers in brushes above and below the LCST. ${ }^{24}$ The change in surface wettability was illustrated using static contact angle measurements of water droplets on PNIPAM brush, ${ }^{25}$ and the surface force apparatus (SFA) ${ }^{26}$ and atomic force microscope $(\mathrm{AFM})^{27}$ were employed to characterize the change in adhesive forces. Though most studies 
only focused on comparing properties above and below the LCST, ${ }^{28}$ there are exceptions ${ }^{29}$ : Lopez et $a l .{ }^{24}$ and Zhang et $a .^{29 \mathrm{~d}}$ studied the conformation of PNIPAM brushes upon continuously increasing/decreasing the temperature and found that the temperature-range of the hydration transition is larger than for PNIPAM in bulk solution. Bureau et al. ${ }^{26}$ systematically studied the adhesive force between PNIPAM brushes near the LCST using SFA. They found that the pull-off force is too small to be measured below the LCST, but continuously increases above the LCST without reaching a plateau (for temperatures below $38^{\circ} \mathrm{C}$ ). The dissipative properties of compressed PNIPAM brushes near the LCST have, however, not been studied so far, despite its relevance in the development of switchable tribo-mechanical systems.

In this paper, we present AFM measurements of the adhesion hysteresis and friction between a high-density PNIPAM brush and a gold colloid in water for different temperatures around the LCST. We observe that these dissipative properties of PNIPAM brushes display a pronounced maximum exactly at the LCST, which results from stretching of the partly collapsed PNIPAM chains when the gold colloid is moved away from the original contact position. The maximum is observed in both the adhesion hysteresis and the friction and is most pronounced when the brush is only gently compressed and when the velocities are small. The frictional response of our system can be tuned over two orders of magnitude by varying the temperature over a range of smaller than 15 degrees. Therefore, our results will aid in the development of "smart" surfaces with tunable tribo-mechanical properties.

\subsection{Materials and Methods}

\subsubsection{Materials}

$N$-isopropylacrylamide (NIPAM, Aldrich, 97\%) was purified by recrystallization twice from toluene/hexane solution $(50 \% \mathrm{v} / \mathrm{v})$, and dried under vacuum for $48 \mathrm{~h}$ at room temperature. Copper (I) bromide ( $\mathrm{CuBr}$, Aldrich, 98\%) was stirred in excessive acetic acid and filtered till the suspension solution is pale like, and dried in vacuum oven at room temperature overnight. $N, N, N^{\prime}, N^{\prime}, N^{\prime}$-pentamethyldiethylenetriamine (PMDETA) (98\%), (3aminopropyl)triethoxysilane, 2-bromo-2-methylpropionyl-bromide (98\%), triethylamine (TEA, $\geq 99 \%$ ), copper (II) bromide ( $\geq 99 \%$ ), Ethylenediaminetetra acetic acid tetrasodium salt di-hydrate (EDTA, 99-102\%) were purchased from Sigma-Aldrich, and used as received without purification. Methanol (absolute) and toluene (AR) were purchased from Biosolve. Hydrogen peroxide $\mathrm{H}_{2} \mathrm{O}_{2}$ and ethanol were purchased from Merck. Water from a MilliQ Advantage A 10 purification system (Millipore, Billerica, Ma, USA) was used. 


\subsubsection{Preparation of polymer brushes}

\section{Initiator Deposition}

The schematic of synthesis of PNIPAM brush is shown in Figure 3.1. Silicon substrates were cut to small pieces (approximately $1 \times 1 \mathrm{~cm}^{2}$ ) from a silicon wafer, and cleaned by Piranha solution $\left(\mathrm{H}_{2} \mathrm{SO}_{4}: \mathrm{H}_{2} \mathrm{O}_{2}=3: 1, \mathrm{v} / \mathrm{v}\right)$, then rinsed extensively with water, ethanol, and dried using a nitrogen stream. (Piranha solution reacts strongly with many organic compounds and should be handled with extreme caution.) Dried substrates and (3aminopropyl)triethoxysilane $(100 \mu \mathrm{l})$ were placed in a desiccator under vacuum for 15 minutes, after which vapor deposition was allowed to proceed overnight. Afterwards, the silicon substrates were washed with ethanol and water sequentially, and dried in a nitrogen stream. In a beaker, a solution of toluene $(20 \mathrm{ml})$ and triethylamine $(0.1 \mathrm{ml})$ was prepared and cooled with ice to $0{ }^{\circ} \mathrm{C}$. Next, the substrates were immersed into the solution with a holder that keeps the samples perpendicularly standing (to avoid precipitated salt adhering to the substrates). 2-Bromo-2-methylpropionly bromide $(0.1 \mathrm{ml})$ was added to the stirring solution drop by drop. The reaction was carried out for $1 \mathrm{~h}$ at room temperature, after which the substrates were rinsed with toluene, and sonicated for $1 \mathrm{~min}$. Lastly, the substrates were washed with ethanol and water, and dried with nitrogen gas.

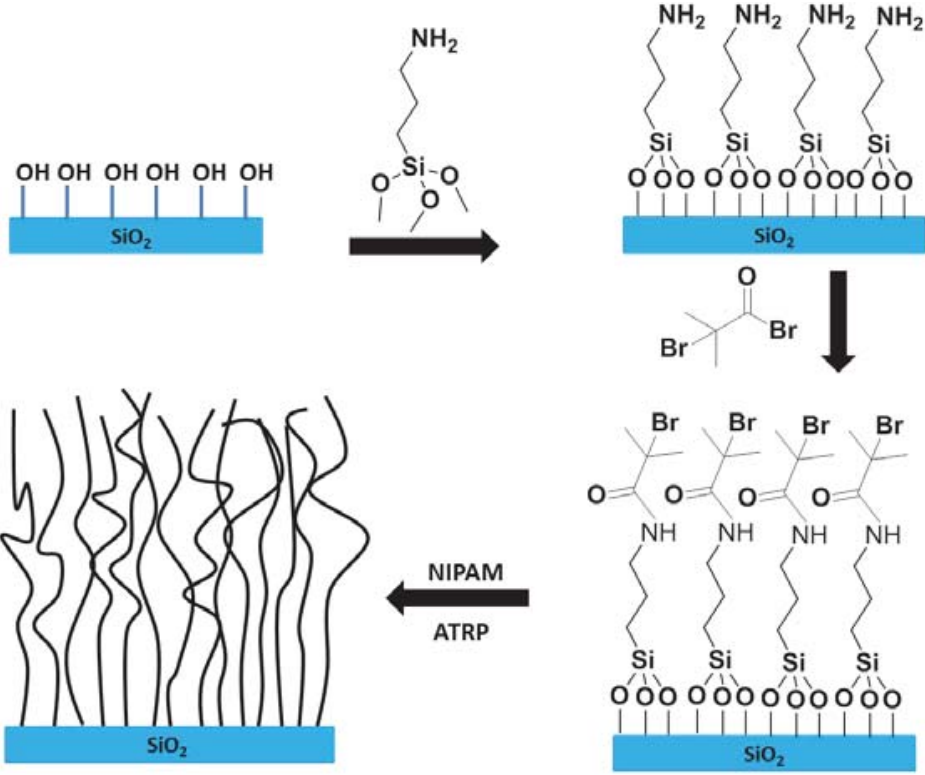

Figure 3.1. Schematic of synthesis of PNIPAM brush on silicon surface using three steps.

\section{ATRP Synthesis of PNIPAM Brushes}

Before polymerization, all the glassware was washed with Piranha solution. In a $25 \mathrm{ml}$ round bottom flask, NIPAM $(2.85 \mathrm{~g}, 25 \mathrm{mmol})$ and PMDETA $(157 \mu \mathrm{l}, 0.5 \mathrm{ml})$ were added 
to a MiliQ water $(9 \mathrm{ml})$ and methanol $(1 \mathrm{ml})$ mixture. Next the mixture was purged with argon for $30 \mathrm{~min}$. Meanwhile, in another flask, $\mathrm{CuBr}(35.8 \mathrm{mg}, 0.25 \mathrm{mmol})$ and $\mathrm{CuBr}_{2}(5.6$ $\mathrm{mg}, 0.025 \mathrm{mmol}$ ) were flushed with argon for 30 minutes. A $20 \mathrm{ml}$ syringe was used to move the monomer and ligand solution to the flask with catalyst. The mixed solution was stirred for 30 minutes and bubbled with argon to form the organometallic complex. The previously modified silicon substrates were sealed in flasks flushed with argon for at least 30 minutes. Later, the solution containing monomer, ligand, and catalyst was transferred to the flask with the substrates. The polymerization was allowed to proceed for 30 minutes at room temperature under argon atmosphere. MilliQ water was injected into the flask to stop the polymerization. Finally, the substrates were rinsed in EDTA solution $(0.1 \mathrm{M})$ to remove the copper, and dried with nitrogen gas. For a demonstration of the sample preparation procedure see Ref. ${ }^{30}$

\subsubsection{Characterization}

Static contact angle measurements were performed by the sessile drop technique using an optical contact angle device equipped with an electronic syringe unit (OCA15, Dataphysics, Germany). The sessile drop was deposited onto the sample surface with the syringe, and the drop contour was fitted by the Young-Laplace method. At least three different measurements on each sample were performed. The contact angle on the sample surface after each functionalization step is shown in Figure 3.2. The measured contact angles on initiator $\left(72.2^{\circ}\right)$ and PNIPAM $\left(58.1^{\circ}\right)$ are in agreement with literature values. ${ }^{31}$

Grazing angle Fourier transform infrared (FTIR) spectroscopy was employed to establish which groups are present in the tethered brushes after polymerization. FTIR spectra were obtained using a Bruker Vertex 70v spectrometer. A background spectrum was obtained by scanning a clean silicon substrate. The results confirm the presence of PNIPAM on the surface (see Figure 3.3). FTIR wavenumbers $\left(\mathrm{cm}^{-1}\right)$ : 3289 (NH stretching vibration), 3078$2874\left(\mathrm{CH}_{2}\right.$ stretching vibration), 1635 ( $\mathrm{C}=\mathrm{O}$ stretching vibration), 1535 (amide II), 14581386 ( $\mathrm{CH}$ deformation vibration), 1366-1170 (CN stretching vibration). The characteristic groups of PNIPAM are $\mathrm{C}=\mathrm{O}$ and $-\mathrm{CN}$ - appeared at 1635 and $1535 \mathrm{~cm}^{-1}$ respectively, which shows there are PNIPAM brushes grafting on the silicon substrates. 
Silicon-OH

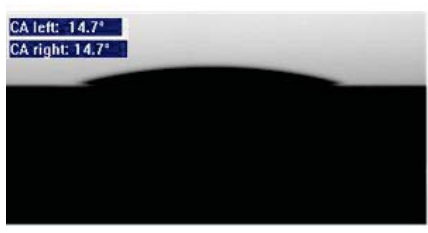

Silicon- $\mathrm{Br}$

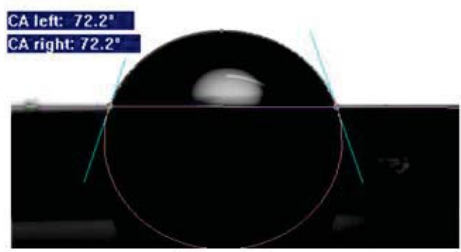

Silicon- $\mathrm{NH}_{2}$

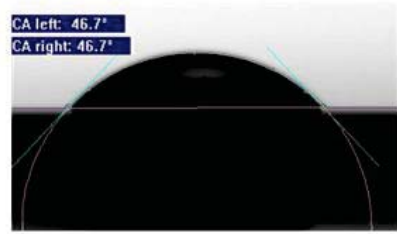

Silicon-PNIPAM

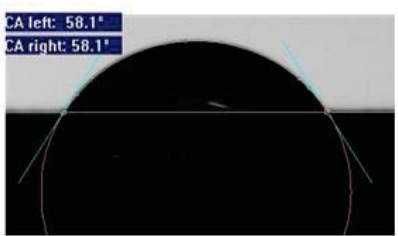

Figure 3.2. The static contact angle of silicon surfaces with various fabrication steps.

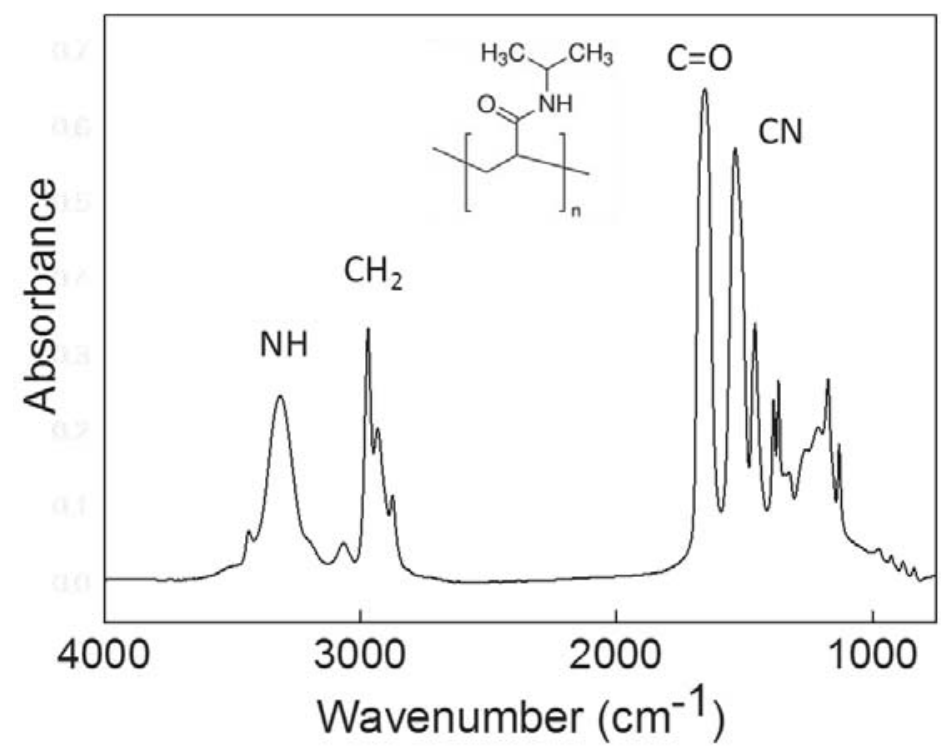

Figure 3.3. FTIR of PNIPAM brushes tethered on silicon surface.

AFM force measurements were performed on a Multimode 8 AFM using a NanoScope V controller, a JV vertical engage scanner and a liquid cell (Bruker, San Barbara, CA). The heater setup is located on the scanner stage below the sample. Gold colloids attached to silicon cantilevers (6 $\mu \mathrm{m}$ diameter, CP-CONT-Au-C, sQube, NanoAndMore, Germany) are used as force probes. Cantilevers without backside coating are used, because they are less sensitive to changes in the surroundings such that we can change the temperature without having to realign the laser. The deflection sensitivity is determined for each temperature. The spring constant (typically $k=2.02 \mathrm{~N} / \mathrm{m} \pm 0.03$ ) is determined in water using the thermal noise method as implemented in the NanoScope 8.15 software. In the adhesion 
hysteresis measurements, we monitor the static deflection of the cantilever upon approach to and retract from surface over $2 \mu \mathrm{m}$ with varying the velocity at $0.75,3.3,6.5$, and 13 $\mu \mathrm{m} / \mathrm{s}$ and various maximum indentation loads $(42,84,168 \mathrm{nN})$ on at least 5 different positions on the sample surface. During the approach the surface is moved towards the colloid until the force equals the maximum indentation load due to deflection of the cantilever. Next, the surface is retracted from the colloid until the starting position is reached. All measurements are conducted in MilliQ water, which was injected into the liquid cell using a syringe. After each measurement, the temperature was increased to the next value at which the system was equilibrated for 20 minutes. The real-time temperature is detected by a calibrated thermocouple with the wire tip directly in contact with the sample. The adhesion force we define as the lowest force of the retract curve. The adhesion hysteresis is calculated by integrating the difference in force upon approach and retract as a function of the piezo displacement. The degree of polymerization $(P=2200$, molar mass $\left.2.5 \pm 1 \times 10^{5} \mathrm{~g} / \mathrm{mol}\right)$ and the grafting density $\left(0.45 \pm 0.2\right.$ chains $\left./ \mathrm{nm}^{2}\right)$ were estimated by swelling ratio. ${ }^{37}$ The uncertainty in the molar mass was estimated using gel permeation chromatography measurements of reference samples of which the PNIPAM was detached using the method of Patil et al., ${ }^{32}$ which yielded molar masses of $1 \times 10^{5}$ for brushes with a dry height of $120 \mathrm{~nm}$. In the Multimode AFM, the temperature is controlled by a thermal stage which can only heat the system above the ambient temperature $\left(26.3{ }^{\circ} \mathrm{C}\right)$. In order to characterize the swelling ratio of the brush over a wider temperature range, and to test if our results are system-independent, another temperature- controlled AFM is employed. For these experiments the height of PNIPAM brush is measured by a Cypher ES Environmental AFM (Asylum Research/Oxford Instruments, Santa Barbara, CA) in contact mode. In contrast to the Multimode AFM, the Cypher ES AFM has a closed environmental chamber that combines accurate measurement of the sample temperature with heating and cooling and excellent temperature stability under various operating conditions. A soft gold colloidal cantilever $(D=6 \mu \mathrm{m}$, shown in Figure $3.4, k=0.33 \mathrm{~N} / \mathrm{m} \pm 0.02)$ is used to measure the heights at different temperatures using a small load $(1.3 \mathrm{nN})$ to minimize indentation into the brush. Again, after each measurement, the temperature is increased to the next temperature after which we waited 20 minutes for the system to reach equilibrium. At least three height images are captured to evaluate the swelling ratio. The friction is measured upon moving the sample in the lateral direction (with the scan angle: $90^{\circ}$ ) over a sliding size $20 \mu \mathrm{m}$ using a velocity of $40 \mu \mathrm{m} / \mathrm{s}$. The cantilever is calibrated using the noncontact method of Vezenov55 resulting in a torsional conversion factor of $S_{\theta}=1.42 \times 10^{-7} \mathrm{~N} / \mathrm{V}$. We repeated our experiments on 5 independently prepared sample surfaces, which all showed qualitatively the same results. 


\subsection{Results and Discussion}

Figure 3.5 shows the swelling ratio (swollen height normalized by the dry brush height) of a PNIPAM brush upon stepwise increasing or decreasing the temperature between 20 and $40{ }^{\circ} \mathrm{C}$. We measure the height of the brush with respect to the bare silicon surface, which is exposed by gently scratching off part of the polymer graft using Teflon tweezers. The measurements are conducted using AFM in imaging mode (over a scan size of $30 \mu \mathrm{m}$, scan rate $1 \mathrm{~Hz})$ with a feedback on the normal load, which is kept small $(1.3 \mathrm{nN})$ to ensure that the brushes are only slightly compressed by the colloid probe.

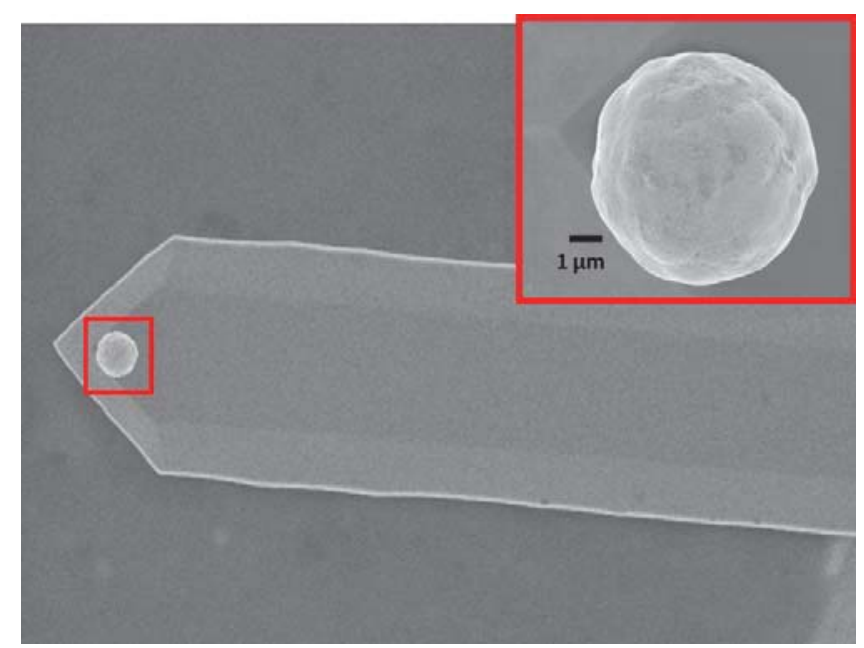

Figure 3.4. SEM image of typical gold colloid cantilever, the diameter is around $6 \mu \mathrm{m}$ (the scale bar is $1 \mu \mathrm{m})$.

As shown in Figure 3.5. (b), at $20^{\circ} \mathrm{C}$ (room temperature) the PNIPAM brush is swollen. The swollen brush height is approximately 2.7 times higher than the height of the dry brush, which is in agreement with typical swelling ratios $(\approx 3)$ reported in the literature for similar grafting densities (from 0.3-0.5 chains $/ \mathrm{nm}^{2}$ ). ${ }^{26,33}$ Around room temperature the brush height only weakly depends on temperature; upon increasing the temperature to $24{ }^{\circ} \mathrm{C}$, the brush height slightly decreases and still shows a swelling ratio of 2.5. Though, this can mean that the brush is already partly collapsed, considering that the volume expansion coefficient for PNIPAM-water mixtures was measured to be positive. ${ }^{34}$ For temperatures above $26{ }^{\circ} \mathrm{C}$, the swelling ratio starts to decrease more strongly with temperature. Over a range of 10 degrees the brush-hydration reduces until the height of the brush reaches a plateau above $35^{\circ} \mathrm{C}$. Our LCST is very close to the LCST observed for PNIPAM free in solution. ${ }^{15-16}$ This is surprising considering that the presence a wall (or colloid) will alter the free energy of the brush, ${ }^{35}$ which was found to shift the LCST for thin brushes. ${ }^{31 a}$ For our system, however, this effect is expected to be small, because the height of our brushes is a lot larger (dry 170 $\mathrm{nm}$ ) than the range of (Van der Waals) interactions (typically $<5 \mathrm{~nm}$ ). 


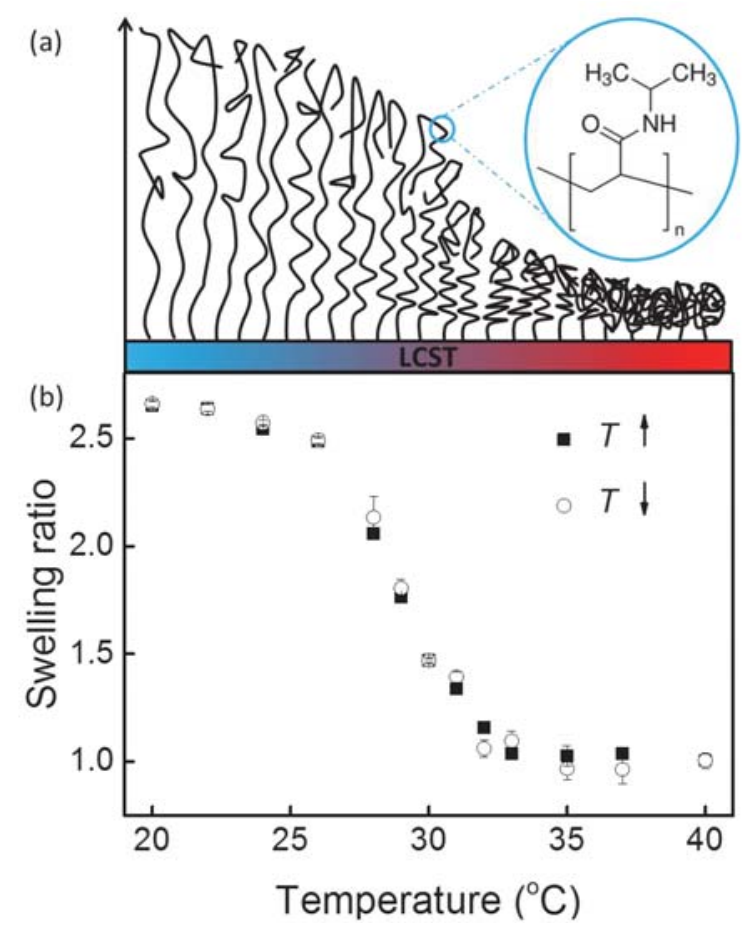

Figure 3.5. Schematic of the temperature induced conformational change of the PNIPAM brush (top) and the swelling ratio of a PNIPAM brush for increasing (solid square) or decreasing (open circle) the temperature of the environment. The swelling ratio is calculated from the swollen height of the brushes normalized by the dry brush height as measured by AFM imaging using a small normal load (1.3 nN).

The gradual decrease of the height with temperature over 10 degrees (Figure 3.5) is in agreement with high-density PNIPAM brush-height measurements by others ${ }^{26,33}$ and is longer-ranged than the temperature range for the coil-to-globule transition of bulk PNIPAM molecules in water (of $4-5{ }^{\circ} \mathrm{C}$ ). ${ }^{36}$ The origin of this broader temperature range for brushes is debated. It can be a result of the polymer-density that gradually decreases as the distance from the surface increases. ${ }^{37}$ Since the LCST depends on the concentration, ${ }^{38}$ different parts of the brush collapse at different temperatures. ${ }^{39}$ It has also been claimed that the broad temperature range of polymer collapse indicates that the coil-to-globule transition of PNIPAM is not a first order phase transition. ${ }^{29 d, 36,40}$ However, others argued that a smeared phase transition temperature range is normal for finite sized systems and does not necessarily indicate that the transition is not a first order phase transition. ${ }^{41}$

At the highest temperature, the height of the collapsed brush is almost the same as the dry brush height such that the swelling ratio is approx. 1.05. This is consistent the swelling ratio observed in AFM experiments by others. ${ }^{33 a}$ However, it is too small considering that for bulk PNIPAM still 3 water molecules are bound to a NIPAM monomer at $40{ }^{\circ} \mathrm{C}$. ${ }^{42}$ Taking into account the effective volume of water molecules and a NIPAM monomer, one expects a swelling ratio of at least 1.2 for such a hydration number. We attribute this discrepancy to 
the AFM imaging conditions in which a positive normal load (of $1.3 \mathrm{nN}$ ) is applied on the brush during imaging. Above the LCST the water is not tightly bound to the PNIPAM ${ }^{43}$ and can easily be squeezed out during imaging such that our apparent swelling ratio is smaller than the swelling ratio for an uncompressed brush.

To test for systematic changes in the response of the PNIPAM brush, we measured the height upon both heating and cooling of the sample. Figure 3.5. (b) shows that the swelling ratio is almost the same (within the experimental error) for the heating and the cooling path, which indicates that the thermal-responsive properties of PNIPAM brushes are reversible and that we heat/cool slow enough to prevent hysteretic behavior. ${ }^{29 \mathrm{~d}, 44}$

Figure 3.6 shows typical force-separation curves measured with the AFM colloid probe upon approach to and retract from the PNIPAM brush at various temperatures. The brush is approached until we reach a maximum normal load of $168 \mathrm{nN}$ after a displacement of $2 \mu \mathrm{m}$ using an approach velocity of $3.3 \mu \mathrm{m} / \mathrm{s}$. The 'zero' is set to be the distance at which the force diverges, which is the separation where the change in deflection is determined by the bending of the cantilever alone and no brush compression occurs. This distance depends on the stiffness of the cantilever and should not be confused with solid-solid contact.

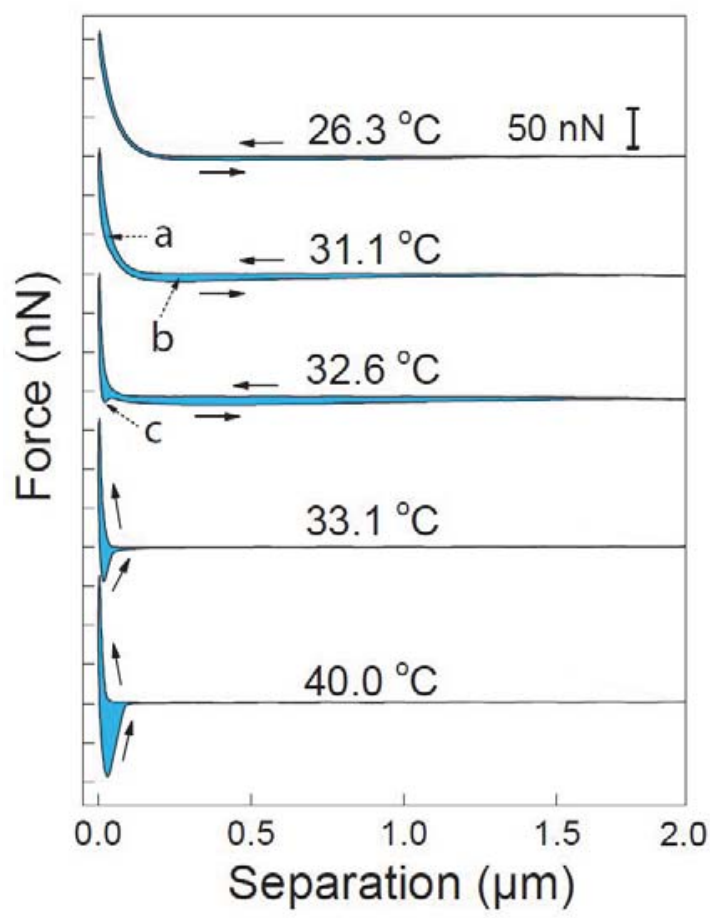

Figure 3.6. Typical Force-Separation curves for the PNIPAM brush at various temperatures. The blue areas denote the difference between the approach and retraction curves, which is a measure for the dissipation per indentation cycle. The scale bar equals $50 \mathrm{nN}$. The full arrows denote the direction of motion. The dashed arrows denote: (a) Squeeze-out force at close contact, (b) long-ranged adhesive interaction, (c) short-ranged adhesion and snap-off. 
At $26.3{ }^{\circ} \mathrm{C}$ (Figure 3.6), we observe that the approach and the retract curves almost overlap. This means that there is little hysteresis and that the work $W$ per indentation cycle is small. Since water is a good solvent for PNIPAM, the osmotic pressure of the solvent is high. Therefore, the brush is only slightly compressed by the colloidal probe and only small amounts of water will be squeezed out, such that there is very little interaction with the colloid and also almost no dissipation.

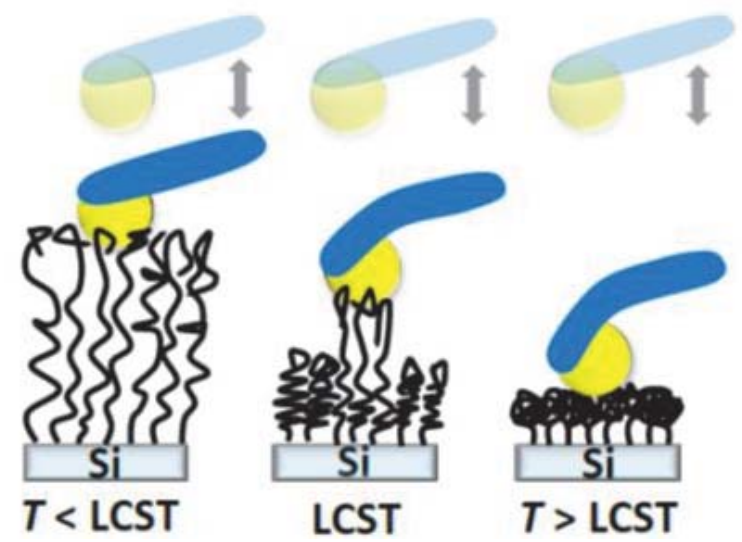

Figure 3.7. Scheme depicting the chain stretching during cantilever retraction from brush surface at temperatures below (left), at (middle) and above (right) the LCST of PNIPAM in water.

Just below the LCST, at $31.1{ }^{\circ} \mathrm{C}$ (see Figure 3.6), the hysteresis increases significantly. The approach curve shows that the force is zero until close contact. Upon retraction, we observe a long-ranged adhesive interaction over approximately $1 \mu \mathrm{m}$. This indicates that the polymers, which are already partly collapsed at this temperature (swelling ratio is only 1.4, see Figure 3.5. (b)), are stretched out over almost $1 \mu \mathrm{m}$ when the probe is retracted, as we schematically depict in Figure 3.7. These results are qualitatively similar to chain-stretching of collapsed charged PAA brushes by a silica colloid. ${ }^{45}$ We note that the number of effective polymer segment-colloid contacts is much higher in our experiments (where we employed a colloid of $6 \mu \mathrm{m}$ diameter) than depicted in Figure 3.7. Assuming a Hertzian contact geometry, we estimate the number of contacts between gold and PNIPAM monomers to be around $2 \times 10^{6}$ at the maximum indentation. The presence of adhesive interaction further increases this number, especially when taking into consideration that the attractive interactions can induce a local collapse. ${ }^{35}$ The low water-content in the brush at $31.1{ }^{\circ} \mathrm{C}$ (shown by the low swelling ratio in Figure 3.5. (b)) allows for more contact between the gold colloid and PNIPAM when the colloid is compressing the brush. Because the PNIPAM polymers stretch over $1 \mu \mathrm{m}$ upon retraction (see Figure 3.6), we can conclude that there must be an attractive interaction between the gold colloidal probe and PNIPAM that is strong enough to allow for replacing the PNIPAM-PNIPAM bonds between the 
collapsed PNIPAM segments by PNIPAM-water hydrogen bonds for the stretched PNIPAM.
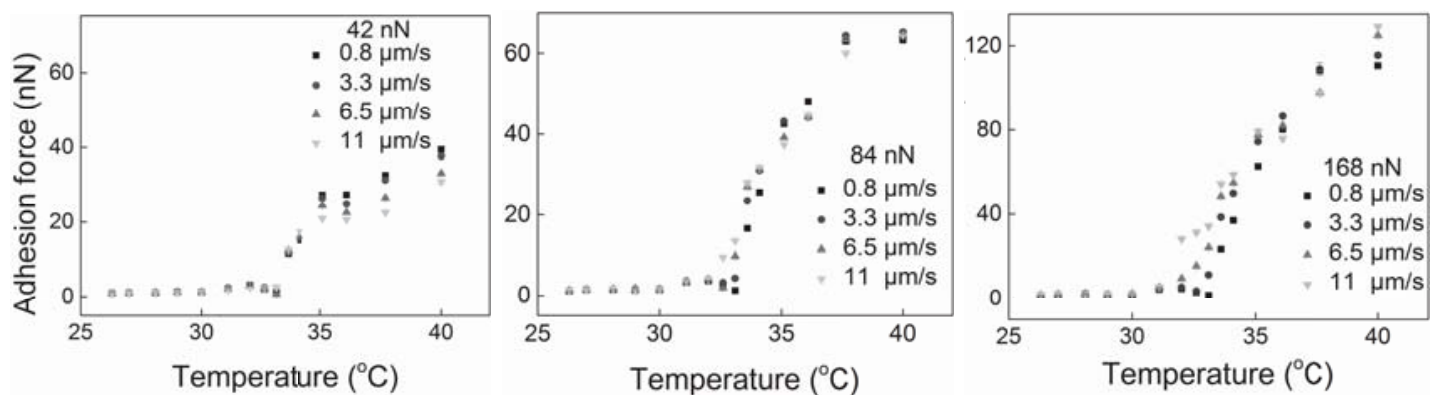

Figure 3.8. Adhesion force as a function of temperature at 4 various velocities ( 0.8 (square), 3.3 (circle), 6.5 (upward triangle) and 11 (downward triangle)) and 3 different normal loads of $42,84,168 \mathrm{nN}$.

At the LCST (the curve at $32.6{ }^{\circ} \mathrm{C}$ in Figure 3.6), we observe a stronger long-ranged adhesive interaction, which means that at this temperature there are more contacts between polymer-segments and gold and/or that the PNIPAM chains resist more strongly against stretching than at lower temperatures. As shown in more detail in Figure 3.8, the minimum in the long-ranged adhesive interaction for $T=32.6{ }^{\circ} \mathrm{C}$ is deeper $(5.5 \mathrm{nN}$ at a separation of $400 \mathrm{~nm}$ ) compared the maximum adherence at $T=31.1{ }^{\circ} \mathrm{C}$ (only $5.1 \mathrm{nN}$ at a separation of $290 \mathrm{~nm}$ ). Moreover, the total range of the interaction has increased from $1.34 \mu \mathrm{m}$ at $T=$ $31.1{ }^{\circ} \mathrm{C}$ to $1.72 \mu \mathrm{m}$ at $T=32.6{ }^{\circ} \mathrm{C}$. This observation is in agreement with the theoretical explanation that for higher temperatures water-amide hydrogen bonds are less favourable due to the higher entropic cost for water-adsorption on the polymer chain, such that intramolecular hydrogen bonds do not want to be replaced by water-amide (inter-molecular) hydrogen bonds upon stretching. At the point where the colloid is still in close contact with the collapsed brush, we also observe a small sharp adhesive minimum. This indicates that for many PNIPAM-segments the PNIPAM-PNIPAM bonds are strong enough to overcome the interaction between PNIPAM and gold such that we get a partial snap-off instability. This speculation could in principle be checked with single-molecule pulling experiments. However, at present, the resolution in the force in these experiments is too low to be able to measure the temperature dependent change in molecular bond strength. ${ }^{46}$ The presence of both the long-ranged adhesive interaction and the sharp adhesive force makes that we find the highest energy dissipation at the LCST $\left(32.6^{\circ} \mathrm{C}\right)$, as depicted in Figure 3.9.

Above the LCST $\left(T=33.1{ }^{\circ} \mathrm{C}\right.$, Figure 3.6), the long-ranged adhesion is negligible. Thus, only very few chains are stretched during retraction. The sharp snap-off adhesive force at close contact is stronger than for lower temperatures. This implies that more chains resist stretching, which would result in a stronger short-ranged adhesive force. Recent single 
molecule experiments of a PNIPAM chain show that, within the experimental accuracy, a collapse single polymer chain can be stretched with just as little effort as a PNIPAM chain in the coil state. ${ }^{46}$ In our brush system, we have many chains in close proximity of each other, which allows for more hydrogen bonds between PNIPAM segments compared to a single, isolated globular PNIPAM chain. Therefore, we are able to observe the effect of temperature-dependent inter- and intramolecular interactions on the adhesive force. Upon further increasing of the temperature to $T=40^{\circ} \mathrm{C}$, the adhesive force increases, which we consider being the result of an increased contact area compared to lower temperatures due to a lower elastic modulus/higher mobility of the PNIPAM chains. The larger contact area allows for more PNIPAM-gold interaction sites.

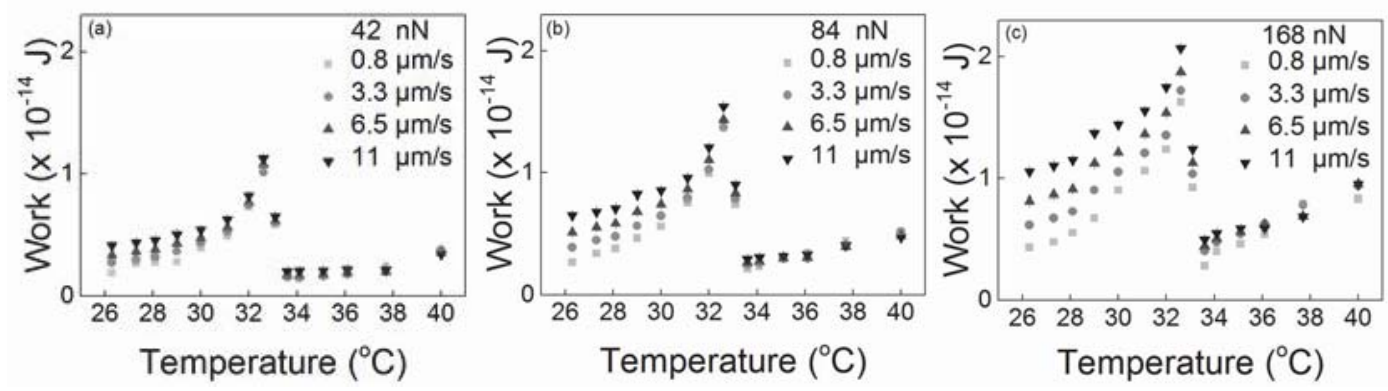

Figure 3.9. Adhesion hysteresis as a function of temperature at various trigger points and velocities. The error bars denote the standard error of the mean using a $95 \%$ confidence interval.

In summary, at low temperatures $\left(T<27{ }^{\circ} \mathrm{C}\right)$, the brush is swollen (the free energy as a function of water-PNIPAM composition will show one minimum). At these temperatures, there is little direct contact between PNIPAM and the gold colloid. The hysteresis in the compression curves is very small and mainly determined by solvent squeeze-out. At temperatures close to the LCST of $32{ }^{\circ} \mathrm{C}$, PNIPAM and water will phase-separate (there are two shallow minima in the free energy vs water-PNIPAM composition). The brush collapses and more PNIPAM-gold contacts can be formed. At these temperatures, the hysteresis is determined by stretching of the partly collapsed PNIPAM molecules upon retract of the colloid from the surface (see Figure 3.6, $T=31.1^{\circ} \mathrm{C}$ and $T=32.6{ }^{\circ} \mathrm{C}$ ), which indicates that the PNIPAM-gold interactions are strong enough to overcome the PNIPAMPNIPAM interactions. At temperatures above the LCST $\left(T>33.0^{\circ} \mathrm{C}\right)$, the brush is fully collapsed (there are two deep minima in the free energy vs water-PNIPAM composition) and many direct PNIPAM-gold contacts can be formed. The hysteresis is determined by a sharp adhesive minimum at close contact. This implies that for $T>33.0{ }^{\circ} \mathrm{C}$, the PNIPAMPNIPAM interactions are strong enough to break the physical bonds between PNIPAM and gold such that the polymers are no longer stretched out. 
Figure 3.9 shows the adhesion hysteresis (work) per approach/retract cycle for various temperatures between 26.3 and $40{ }^{\circ} \mathrm{C}$, measured at 4 different ramp velocities ( 0.8 (square), 3.3 (circle), 6.5 (upward triangle), 11 (downward triangle) $\mu \mathrm{m} / \mathrm{s}$ ), and 3 different maximum normal loads of (a) 42, (b) 84 and (c) $168 \mathrm{nN}$. For each data point, at least 20 forceseparation curves are obtained. All these datasets show a similar relation between the work and temperature. With increase of temperature, the dissipation gradually increases. At the LCST, there is the highest dissipation due to strong stretching of PNIPAM chains upon retraction of the gold probe (as schematically illustrated in Figure 3.7). Above the LCST, the dissipation is lower for all velocities and loads used in these experiments, because it is caused by the short-ranged adhesive (snap-out-of-contact) instability alone. At temperatures of $33-34{ }^{\circ} \mathrm{C}$, this short-ranged adhesive force is still small $\left(10-20 \%\right.$ of the force at $40{ }^{\circ} \mathrm{C}$, as we show in Figure 3.8) and thus there is little dissipation. With further increase of temperature, the adhesive force for $T>$ LCST increases with temperature for all parametersettings. This trend becomes more pronounced under higher loads.

Figure 3.9. (a) shows that for $T<$ LCST the work depends weakly (approximately logarithmically) on the velocity. At $26.3{ }^{\circ} \mathrm{C}$, the work for $11.2 \mu \mathrm{m} / \mathrm{s}$ is $2.4 \mathrm{x}$ as high as for $0.8 \mu \mathrm{m} / \mathrm{s}$ for the maximum normal load of $168 \mathrm{nN}$. For temperatures above the LCST the work is independent of the velocity within the experimental error. Examination of the force traces shows that the (long-ranged) adhesive interaction is independent of the velocity, while the squeeze-out forces upon compression and decompression of the brush in close contact are velocity-dependent. At higher temperatures the (long-ranged) adhesive contribution to the dissipation dominates over the dissipation due to solvent squeeze-out such that the work becomes independent of the velocity for higher temperatures.

Comparison of Figure 3.9. (a), (b) and (c) shows that the maximum applied load also significantly affects the dissipation. For a low normal load $(42 \mathrm{nN})$, there is less solvent squeeze-out at lower temperatures. Therefore, the hysteresis is at all temperatures mainly determined by chain stretching. The maximum in the work is most pronounced at this normal load $(42 \mathrm{nN})$; the work at $32.6{ }^{\circ} \mathrm{C}$ is $5.5 \mathrm{x}$ higher than at $26.3{ }^{\circ} \mathrm{C}$ for a low velocity. For high loads $(168 \mathrm{nN})$, the work increases for all temperatures. However, below the LCST the work increases more strongly than at higher temperatures such that the maximum in the work is less pronounced; for a maximum load of $168 \mathrm{nN}$, the work at $32.6{ }^{\circ} \mathrm{C}$ is only $3.7 \mathrm{x}$ higher than at $26.3{ }^{\circ} \mathrm{C}$ for $v=0.8 \mu \mathrm{m} / \mathrm{s}$. The reason for this is that at lower temperatures, the effect of solvent squeeze-out is more important. This strongly increases the dissipation at temperatures below $32{ }^{\circ} \mathrm{C}$, while at higher temperatures the hysteresis is still mainly determined by PNIPAM-gold interactions (resulting in stretching of the chains at the LCST). The latter depends less strongly on the applied load.

We observed a dissipative maximum for all employed approach velocities and maximum normal loads. Nevertheless, the observation that the height of the maximum depends on the normal load and approach velocity implies that the presence of a dissipative maximum at 
the LCST is not guaranteed for all velocities and maximum normal loads. For very high velocities and high normal loads, there might not be a dissipative maximum at the LCST. Moreover, the dominance of PNIPAM stretching to the dissipation and therefore the presence of the dissipative maximum will depend on the grafting density and degree of polymerization such that the presence of a maximum in the work will also depend on these parameters. We have obtained qualitatively similar results for a silicon oxide probe in combination with a PNIPAM brush. Thus, our results are not specific to gold colloids in relative motion with PNIPAM and will hold provided that the interaction between the colloid and brush is strong enough to allow for chain stretching.

If the maximum in the dissipation at the LCST can be attributed to stretching of the polymers, we expect a similar frictional response of the system upon sliding in the lateral direction. The change in friction between a colloid and PNIPAM brush or gel below and above the LCST have been studied extensively. ${ }^{47}$ However, little attention has been paid to the evolution of the tribo-mechanical properties of PNIPAM upon changing the temperature in small steps through the LCST $\left(32^{\circ} \mathrm{C}\right)$. Figure 3.10 shows the friction force measured while moving the PNIPAM surface with respect to the colloid in the lateral direction over a distance of $20 \mu \mathrm{m}$ at a velocity of $20 \mu \mathrm{m} / \mathrm{s}$ under a normal load of $2.2 \mathrm{nN}$. We measure the lowest friction force $(0.2 \mathrm{nN})$ at the lowest temperature $\left(20^{\circ} \mathrm{C}\right)$, where the brush swells the most. At these low temperatures, the brush is strongly hydrated resulting in little contact between colloid and PNIPAM. Consequently, the frictional response is low.

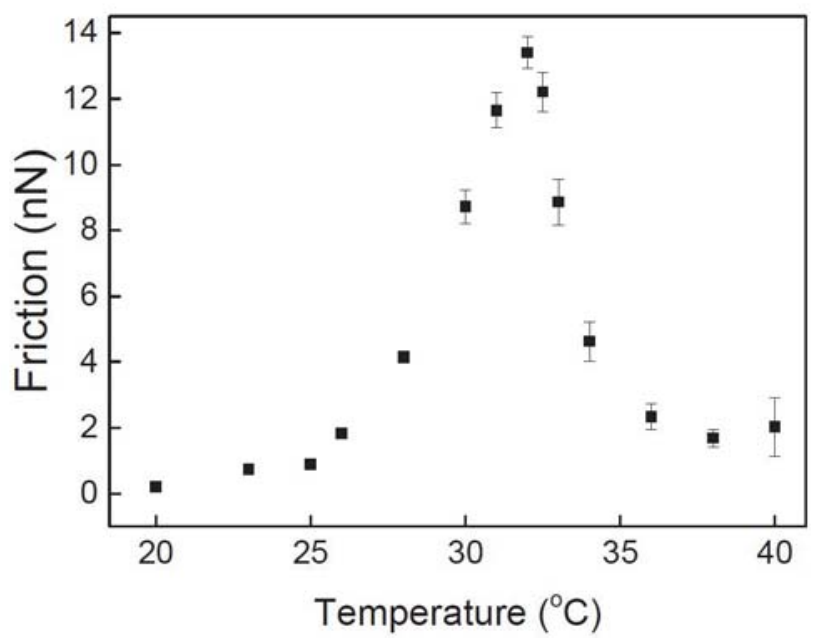

Figure 3.10. Friction force as a function of temperature at scanning velocity of $20 \mu \mathrm{m} / \mathrm{s}$ and normal load of $2.2 \mathrm{nN}$. The error bars denote the standard error of the mean using a 95\% confidence interval.

Upon increasing the temperature towards the LCST, the sliding friction gradually increases. At these higher temperatures, the brush gradually collapses, which allows for more gold- 
PNIPAM contact. When the probe moves in the lateral direction, the polymers in the brush can be stretched again (see Figure 3.11), just as for the adhesion experiments. This results in a high friction at the LCST, which is appox. 100x higher than the force at $20^{\circ} \mathrm{C}$. Above the LCST $\left(T>35{ }^{\circ} \mathrm{C}\right)$, the polymers strongly resist stretching such that the colloid just slides over the surface, which results in lower friction. Interestingly, the strength of the friction force correlates with the presence of oscillations in the force traces, which we propose to be Schallamach waves. ${ }^{48}$

In agreement with most experimental observations by others, ${ }^{47 a, 47 \mathrm{c}-\mathrm{e}}$ we find that the friction above the LCST $\left(40^{\circ} \mathrm{C}\right)$ is 10 times larger than the friction below the LCST $\left(20^{\circ} \mathrm{C}\right)$, which is a result of the increased PNIPAM-colloid interactions. In contrast, Nordgren et al. reported a lower friction above the LCST than below the LCST, which they claimed to arise due to a smaller contact area after collapse. ${ }^{47 b} \mathrm{We}$ propose a different mechanism for their observation. Nordgren et al. performed their 'room temperature' experiments at temperature around $24{ }^{\circ} \mathrm{C}$, where we find that the dissipation is already more than 5 times higher compared to $20{ }^{\circ} \mathrm{C}$. Therefore, we speculate that their results might have been influenced by chain stretching resulting in a higher friction force. Our results and interpretation of the dissipative maximum at the LCST might also provide insights into the dissipative maximum observed by Wang et al. for a thermally responsive hydrogel made of DMAEMA and benzophenone methacrylate. ${ }^{49}$

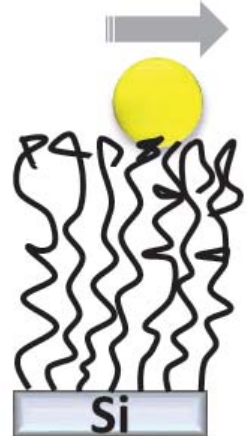

$T<$ LCST

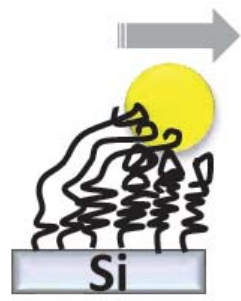

LCST

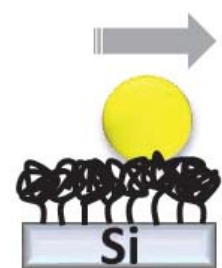

$T>$ LCST

Figure 3.11. Schematic illustration of friction measurement at temperatures below (left), at (middle) and above (right) the LCST of PNIPAM in water.

Some researchers proposed that there is an intermediate state for PNIPAM at the LCST, 33 a, 50 in which PNIPAM can be considered to be in an 'amorphous' rubbery phase. For systems with a temperature dependent solid, rubbery and liquid phase, one expects the dissipation to display a maximum when the inverse shear-rate matches the intrinsic relaxation time of the system. ${ }^{51}$ In accordance with the time-temperature superposition principle, this would mean that the temperature at which one observes the dissipative maximum depends on the shear 
rate (velocity of the colloid). In our experiments, the dissipative maximum occurs, however, at the same temperature for all velocities. Therefore, the presence of such an intermediate phase cannot explain our observations.

\subsection{Conclusions}

In summary, we have shown that the dissipative properties of a PNIPAM brush in relative motion with a gold colloid probe change non-monotonically upon increasing the temperature. Our AFM experiments exposed that both the adhesion hysteresis and the friction display a maximum in the dissipated energy exactly at the LCST. Examination of the force traces revealed that around the LCST preferential interactions between the colloid and PNIPAM results in stretching of the polymer-chains when the colloid is moved away from the initial contact. Our results have important implications for the development of polymer-based switchable tribo-mechanical systems (such as responsive tweezers, friction detectors or artificial muscles), where chain-stretching around the phase transition can induce high dissipation.

\subsection{References}

[1] M. A. C. Stuart, W. T. S. Huck, J. Genzer, M. Muller, C. Ober, M. Stamm, G. B. Sukhorukov, I. Szleifer, V. V. Tsukruk, M. Urban, F. Winnik, S. Zauscher, I. Luzinov and S. Minko, Nature Materials 2010, 9, 101113.

[2] a) I. Tokareva, S. Minko, J. H. Fendler and E. Hutter, Journal of the American Chemical Society 2004, 126, 15950-15951; b) H. Merlitz, G.-L. He, C.-X. Wu and J.-U. Sommer, Physical review letters 2009, 102, 115702.

[3] a) J. P. Gong, Soft Matter 2006, 2, 544-552; b) Y. L. Yu, B. D. Kieviet, E. Kutnyanszky, G. J. Vancso and S. de Beer, Acs Macro Letters 2015, 4, 75-79.

[4] a) M. E. Harmon, M. Tang and C. W. Frank, Polymer 2003, 44, 4547-4556; b) Y. Osada, H. Okuzaki and H. Hori, Nature 1992, 355, 242-244; c) B. D. Kieviet, P. M. Schön and G. J. Vancso, Lab on a Chip 2014, 14, 4159-4170.

[5] X. Feng, X. Sui, M. A. Hempenius and G. J. Vancso, Journal of the American Chemical Society 2014, 136, 7865-7868.

[6] T. Wu, P. Gong, I. Szleifer, P. Vlcek, V. Šubr and J. Genzer, Macromolecules 2007, 40, 8756-8764.

[7] M. Irie, A. Menju and K. Hayashi, Macromolecules 1979, 12, 1176-1180.

[8] H. J. Dai, Q. Chen, H. L. Qin, Y. Guan, D. Y. Shen, Y. Q. Hua, Y. L. Tang and J. Xu, Macromolecules 2006, 39, 6584-6589.

[9] M. Obata, M. Morita, K. Nakase, K. Mitsuo, K. Asai, S. Hirohara and S. Yano, Journal of Polymer Science Part A: Polymer Chemistry 2007, 45, 2876-2885.

[10] C. de las Heras Alarcón, T. Farhan, V. L. Osborne, W. T. Huck and C. Alexander, Journal of Materials Chemistry 2005, 15, 2089-2094.

[11] O. Azzaroni, A. A. Brown and W. T. Huck, Angewandte Chemie 2006, 118, 1802-1806.

[12] Y. Xu, S. Bolisetty, M. Drechsler, B. Fang, J. Yuan, M. Ballauff and A. H. Müller, Polymer 2008, 49 , 3957-3964.

[13] H.-N. Lee and T. P. Lodge, The Journal of Physical Chemistry Letters 2010, 1, 1962-1966.

[14] a) X. Laloyaux, B. Mathy, B. Nysten and A. M. Jonas, Langmuir 2009, 26, 838-847; b) J. F. Lutz, Journal of Polymer Science Part A: Polymer Chemistry 2008, 46, 3459-3470.

[15] a) R. Liu, M. Fraylich and B. R. Saunders, Colloid and Polymer Science 2009, 287, 627-643; b) T. G. McKenzie, E. H. Wong, Q. Fu, S. J. Lam, D. E. Dunstan and G. G. Qiao, Macromolecules 2014, 47, 78697877. 
[16] M. Heskins and J. E. Guillet, Journal of Macromolecular Science: Part A - Chemistry 1968, 2, 14411455.

[17] a) F. M. Winnik, H. Ringsdorf and J. Venzmer, Macromolecules 1990, 23, 2415-2416; b) Y. Zhang, S. Furyk, D. E. Bergbreiter and P. S. Cremer, Journal of the American Chemical Society 2005, 127, 1450514510.

[18] S. T. Milner, Science 1991, 251, 905-914.

[19] a) M. Chen, W. H. Briscoe, S. P. Armes and J. Klein, science 2009, 323, 1698-1701; b) S. Lee and N. D. Spencer, SCIENCE-NEW YORK THEN WASHINGTON- 2008, 319, 575; c) S. de Beer and M. H. Muser, Macromolecules 2014, 47, 7666-7673.

[20] a) G. Z. Gao, K. Yu, J. Kindrachuk, D. E. Brooks, R. E. W. Hancock and J. N. Kizhakkedathu, Biomacromolecules 2011, 12, 3715-3727; b) W. J. Yang, T. Cai, K.-G. Neoh, E.-T. Kang, G. H. Dickinson, S. L.-M. Teo and D. Rittschof, Langmuir 2011, 27, 7065-7076.

[21] X. F. Sui, Q. Chen, M. A. Hempenius and G. J. Vancso, Small 2011, 7, 1440-1447.

[22] X. Sui, X. Feng, J. Song, M. A. Hempenius and G. J. Vancso, Journal of materials chemistry 2012, 22, 11261-11267.

[23] a) S. de Beer, Langmuir 2014, 30, 8085-8090; b) Q. Chen, E. S. Kooij, X. F. Sui, C. J. Padberg, M. A. Hempenius, P. M. Schon and G. J. Vancso, Soft Matter 2014, 10, 3134-3142.

[24] S. Balamurugan, S. Mendez, S. S. Balamurugan, M. J. O'Brie and G. P. López, Langmuir 2003, 19, 2545-2549.

[25] T. L. Sun, G. J. Wang, L. Feng, B. Q. Liu, Y. M. Ma, L. Jiang and D. B. Zhu, Angewandte ChemieInternational Edition 2004, 43, 357-360.

[26] I. B. Malham and L. Bureau, Langmuir 2010, 26, 4762-4768.

[27] D. M. Jones, J. R. Smith, W. T. S. Huck and C. Alexander, Advanced Materials 2002, 14, 1130-1134.

[28] a) G. Q. Liu, X. L. Wang, F. Zhou and W. M. Liu, Acs Applied Materials \& Interfaces 2013, 5, 1084210852; b) K. Jalili, F. Abbasi and A. Milchev, Macromolecules 2013, 46, 5260-5278; c) D. Jańczewski, N. Tomczak, J. Song, H. Long, M.-Y. Han and G. J. Vancso, Journal of Materials Chemistry 2011, 21, 64876490; d) S. de Beer, E. Kutnyanszky, P. M. Schon, G. J. Vancso and M. H. Muser, Nature Communications 2014, 5, 3781; e) E. Svetushkina, N. Puretskiy, L. Ionov, M. Stamm and A. Synytska, Soft Matter 2011, 7 , 5691-5696; f) O. Tagit, N. Tomczak and G. J. Vancso, Small 2008, 4, 119-126; g) H. Yim, M. Kent, D. Huber, S. Satija, J. Majewski and G. Smith, Macromolecules 2003, 36, 5244-5251; h) E. M. Benetti, S. Zapotoczny and J. Vancso, Advanced Materials 2007, 19, 268-271.

[29] a) H. Yim, M. Kent, S. Mendez, G. Lopez, S. Satija and Y. Seo, Macromolecules 2006, 39, 3420-3426; b) M. A. Cole, N. H. Voelcker, H. Thissen, R. G. Horn and H. J. Griesser, Soft Matter 2010, 6, 2657-2667; c) D. Magerl, M. Philipp, X.-P. Qiu, F. o. M. Winnik and P. Müller-Buschbaum, Macromolecules 2015; d) G. M. Liu and G. Z. Zhang, Journal of Physical Chemistry B 2005, 109, 743-747.

[30] S. de Beer, E. Kutnyanszky, M. H. Muser and G. J. Vancso, Jove-Journal of Visualized Experiments 2014, 52285.

[31] a) K. N. Plunkett, X. Zhu, J. S. Moore and D. E. Leckband, Langmuir 2006, 22, 4259-4266; b) D. M. Jones, A. A. Brown and W. T. Huck, Langmuir 2002, 18, 1265-1269.

[32] C. Cummings, H. Murata, R. Koepsel and A. J. Russell, Biomaterials 2013, 34, 7437-7443.

[33] a) S. B. Rahane, J. A. Floyd, A. T. Metters and S. M. Kilbey, Advanced Functional Materials 2008, 18, 1232-1240; b) E. Bittrich, S. Burkert, M. Muller, K. J. Eichhorn, M. Stamm and P. Uhlmann, Langmuir 2012, $28,3439-3448$.

[34] M. Philipp, K. Kyriakos, L. Silvi, W. Lohstroh, W. Petry, J. K. Krüger, C. M. Papadakis and P. MüllerBuschbaum, The Journal of Physical Chemistry B 2014, 118, 4253-4260.

[35] a) S. Alexander, Journal De Physique 1977, 38, 983-987; b) R. Descas, J. U. Sommer and A. Blumen, Macromolecular Theory and Simulations 2008, 17, 429-453.

[36] C. Wu and X. H. Wang, Physical Review Letters 1998, 80, 4092-4094.

[37] S. T. Milner, T. A. Witten and M. E. Cates, Macromolecules 1988, 21, 2610-2619.

[38] I. Idziak, D. Avoce, D. Lessard, D. Gravel and X. Zhu, Macromolecules 1999, 32, 1260-1263.

[39] a) A. Halperin, The European physical journal B-Condensed matter and complex systems 1998, 3, 359364; b) V. A. Baulin and A. Halperin, Macromolecular theory and simulations 2003, 12, 549-559.

[40] M. Philipp, U. Muller, R. Aleksandrova, R. Sanctuary, P. Muller-Buschbaum and J. K. Kruger, Soft Matter 2013, 9, 5034-5041.

[41] G. Graziano, International journal of biological macromolecules 2000, 27, 89-97.

[42] H. Kogure, S. Nanami, Y. Masuda, Y. Toyama and K. Kubota, Colloid and Polymer Science 2005, 283, $1163-1171$.

[43] Y. Okada and F. Tanaka, Macromolecules 2005, 38, 4465-4471.

[44] J. N. Kizhakkedathu, R. Norris-Jones and D. E. Brooks, Macromolecules 2004, 37, 734-743. 
[45] A. Drechsler, A. Synytska, P. Uhlmann, M. M. Elmahdy, M. Stamm and F. Kremer, Langmuir 2010, 26 , 6400-6410.

[46] E. Kutnyanszky and G. J. Vancso, European Polymer Journal 2012, 48, 8-15.

[47] a) X. Banquy, E. Charrault and S. Giasson, The Journal of Physical Chemistry B 2010, 114, 9721-9728;

b) N. Nordgren and M. W. Rutland, Nano Letters 2009, 9, 2984-2990; c) D. P. Chang, J. E. Dolbow and S. Zauscher, Langmuir 2007, 23, 250-257; d) A. Dedinaite, E. Thormann, G. Olanya, P. M. Claesson, B. Nystrom, A. L. Kjoniksen and K. Z. Zhu, Soft Matter 2010, 6, 2489-2498; e) Y. Wu, M. R. Cai, X. W. Pei, Y. M. Liang and F. Zhou, Macromolecular Rapid Communications 2013, 34, 1785-1790; f) A. A. Pitenis, J. M. Uruena, K. D. Schulze, R. M. Nixon, A. C. Dunn, B. A. Krick, W. G. Sawyer and T. E. Angelini, Soft Matter 2014, 10, 8955-8962.

[48] Schallam.A, Wear 1971, 17, 301-312.

[49] J. Huang, B. Cusick, J. Pietrasik, L. Wang, T. Kowalewski, Q. Lin and K. Matyjaszewski, Langmuir 2007, 23, 241-249.

[50] a) X. Wang, X. Qiu and C. Wu, Macromolecules 1998, 31, 2972-2976; b) C. Wu and S. Zhou, Macromolecules 1995, 28, 5388-5390.

[51] a) E. Tocha, H. Schönherr and G. J. Vancso, Soft Matter 2009, 5, 1489-1495; b) H. Yoshizawa, Y. L. Chen and J. Israelachvili, The Journal of Physical Chemistry 1993, 97, 4128-4140. 



\section{Chapter 4}

\section{Tunable Friction by Employment of Co-non- solvency of PNIPAM Brushes*}

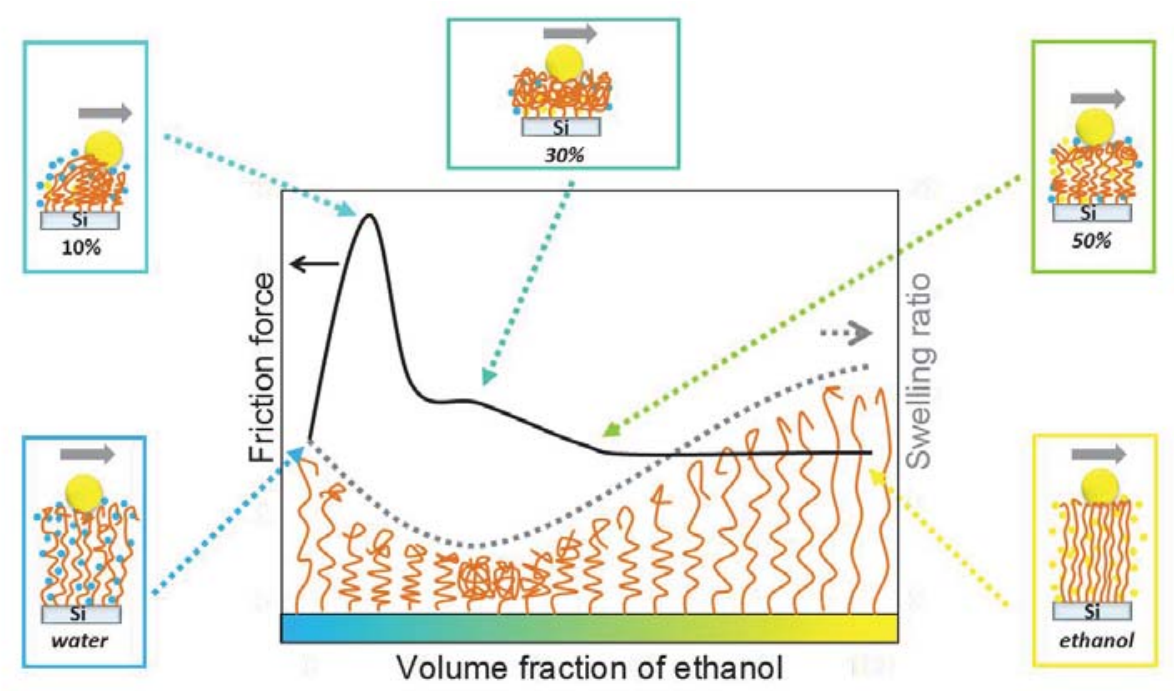

We present a simple method to control the tribological properties of contacts between polymer brushes and a solid counter surface. We show, using atomic force microscopy (AFM) experiments, that the friction force, upon relative sliding of a poly $(N$-isopropyl acrylamide) (PNIPAM) brush and a gold colloid on an AFM cantilever, varies over two orders of magnitude when changing the composition of an ethanol-water mixture that solvates the brush. We achieve this large variation in friction via a co-non-solvency effect: In pure water or ethanol, the PNIPAM brush is swollen and the friction is low, while in a $10-90$ vol \% ethanol-water mixture, the brush is partly collapsed and polymer-stretching causes the friction to be high.

* This chapter has been published in: Yu, Y.; Cirelli, M.; Kieviet, B. D.; Kooij, E. S.; Vancso, G. J.; de Beer, S., Polymer. 2016, 102, 372-378. 


\subsection{Introduction}

Friction between surfaces in relative motion is generally required to be either high or low: When walking, braking or picking up material, sufficiently high friction is of critical importance, while smooth sliding is preferred in systems where friction results in major costs due to energy-loss. However, for some advanced applications, it is necessary to be able to switch between high and low friction. Examples include robots that can walk on walls ${ }^{1}$ and smart tweezers. ${ }^{2}$ Polymer brushes provide a versatile platform for controlling surface properties ${ }^{3}$ including tribo-mechanical properties ${ }^{3 b, 4}$ and, therefore, hold great potential for applications as surfaces with variable (switchable) friction. ${ }^{5}$

Polymer brushes can be created by end-anchoring polymers to a surface or interface at an adequately high density. ${ }^{6}$ When brushes are dry or kept in a poor solvent in their collapsed state, the polymers form a dense layer on the surface. The adhesion and friction between these dense polymer films and a solid counter-surface are generally high. ${ }^{7}$ When a polymer brush is kept in a good solvent, the polymers stretch out and absorb the solvent into brush. Under these conditions, the friction and adhesion are typically low. ${ }^{8}$ By employing polymers that respond to an applied external stimulus (e.g. by changes in temperature, ${ }^{9}$ solvents, ${ }^{10} \mathrm{pH},{ }^{11}$ electric ${ }^{12}$ and magnetic field ${ }^{13}$ etc.), the effective solvent-quality can be readily changed such that friction and adhesion can be controlled. ${ }^{4 a}, 8,14$ A particularly easy method to change the solvent quality is the employment of the so-called co-non-solvency of the polymers ${ }^{15}$ where a coil-to-globule transition of polymers can be triggered by simply adding a relatively small amount of co-solvent to the solvent.

When polymers are in a swollen, coiled state in pure solvent or co-solvent, but form a collapsed globule in a mixture of the two solvents, it is called a co-non-solvency effect. A well-studied example is poly( $N$-isopropyl acrylamide) (PNIPAM) in a mixture of water and alcohols such as methanol, ethanol or isopropanol. ${ }^{16}$ At room temperature, PNIPAM polymers are found to be swollen in water or one of these alcohols, while the polymers are collapsed e.g. for 10-20 vol.\% methanol in a methanol-water mixture for polymers free in solution $^{10}$ (at $30 \mathrm{vol} \%$ for gels and brushes ${ }^{15 b, 17}$ ). The origin of co-non-solvency is still under debate. ${ }^{15 \mathrm{c},} 18 \mathrm{Koga}$ et al. ${ }^{15 \mathrm{c}}$ proposed that co-non-solvency is a result of competitive hydrogen bonding where bonds between water and alcohol-molecules can be favored over hydrogen bonds between PNIPAM and the solvent-molecules. In a different study, Sheng et $a l .{ }^{18 \mathrm{a}}$ conclude from their Fourier transform infrared spectroscopy experiments that the formation of "water-methanol clusters" $\left(\mathrm{H}_{2} \mathrm{O}\right)_{\mathrm{n}}(\mathrm{MeOH})_{\mathrm{m}}$ of concentration-dependent size (n $=1,2,3,4,5 ; \mathrm{m}=1)$ induces a coil-globule-coil transition. Depending on their size, these clusters create either good or poor solvent conditions for PNIPAM. Recently, Mukherji et $a l .{ }^{19}$ reported that the Flory-Huggins theory cannot explain the co-non-solvency mechanism. They show using coarse-grained molecular dynamics simulations ${ }^{18 \mathrm{~b}}$ that a possible explanation for the phenomenon might be that at low concentration of the better solvent, 
bridges are formed between parts of the PNIPAM polymer by the better solvent, which induces a collapse. Nevertheless, irrespective of the exact explanation for the origin, the manifestation of the phenomenon of co-non-solvency is well established and has been

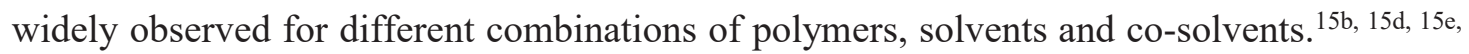

${ }^{20}$ Moreover, it has been employed in the development of applications such as gating ${ }^{21}$ and in alcohol sensing. ${ }^{22}$ However, for tuning friction, it has been relatively unexplored.

In this article we show using friction force atomic force microscopy (AFM) experiments, that co-non-solvency of PNIPAM in ethanol-water mixtures can be used to tune friction over two orders of magnitude. In a recent publication Chen et al. ${ }^{8}$ reported on obtaining variable friction between PNIPAM and a silicon colloid using co-non-solvency. They observe that, in a 50-50 vol. \% mixture of methanol and water, the friction coefficient is approximately 4 times higher than in pure water. We built on these results and study the frictional response upon sliding a gold colloid over a PNIPAM brush at room temperature, while we systematically vary the relative amount of ethanol in ethanol-water mixtures. We find that for $10 \%$ of ethanol, the friction is the highest. At this concentration the friction is $120 \pm 10$ times higher than in pure ethanol. Surprisingly, we find that the concentration for the highest friction force does not coincide with the concentration of minimal brush swelling at $30 \%$ of ethanol. Thus, the observed frictional response cannot be explained by brush-swelling alone. Instead, we show, using AFM measurements in which the colloid probe is approached towards and retracted from the surface, that the partly collapsed polymers in $10 \%$ ethanol have become strongly stretched when the colloid is moved away from its initial contact with the brush. This can explain the enhanced frictional response found at 10 vol.\% of ethanol in the mixture. Because our results show that the friction can be varied over two orders of magnitude, we propose that PNIPAM in these solvent-cosolvent mixtures holds great potential for application in e.g. robots that can walk on walls or smart tweezers.

\subsection{Materials and Methods}

\subsubsection{Materials}

$\mathrm{N}$-Isopropylacrylamide (NIPAM, Aldrich, 97\%) was dissolved in toluene/hexane solution $(50 \% \mathrm{v} / \mathrm{v})$, and recrystallized twice in the refrigerator, then dried under vacuum for $48 \mathrm{~h}$ at room temperature. Copper (I) bromide ( $\mathrm{CuBr}$, Aldrich, 98\%) was purified by stirring in excessive acetic acid and filtered until the suspension solution is light grey, and dried under approximate vacuum conditions at room temperature overnight. $\quad N, N, N^{\prime}, N^{\prime}, N^{\prime}-$ pentamethyldiethylenetriamine (PMDETA) (98\%), (3-aminopropyl) triethoxysilane, 2bromo-2-methylpropionyl bromide (98\%), triethylamine (TEA, $\geq 99 \%$ ), copper (II) bromide ( $\geq 99 \%$ ), ethylenediaminetetra acetic acid tetrasodium salt di-hydrate (EDTA, 99$102 \%$ ) were purchased from Sigma-Aldrich, and used as received without purification. 
Methanol (absolute), sulfuric acid (95-97\%), tetrahydrofuran (THF), hexane and toluene (AR) were purchased from Biosolve, and hydrogen peroxide $\mathrm{H}_{2} \mathrm{O}_{2}$ and ethanol were purchased from Merck and used as received. MilliQ water was obtained from a MilliQ Advantage A 10 purification system (Millipore, Billerica, Ma, USA).

\subsubsection{Brush preparation and characterization}

The PNIPAM brushes were grafted from pieces of a silicon wafer $\left(1 \times 1 \mathrm{~cm}^{2}\right)$ via surfaceinitiated ATRP. ${ }^{4 a}$ First, the silicon wafer was cleaned by Piranha solution $\left(\mathrm{H}_{2} \mathrm{SO}_{4}: \mathrm{H}_{2} \mathrm{O}_{2}=\right.$ $3: 1, \mathrm{v} / \mathrm{v}$ ). (Caution: Piranha is very dangerous, extreme caution should be taken when handling it.) Next, a monolayer with an amine group ((3-aminopropyl)triethoxysilane) was attached through vapor deposition. Then, the initiator was attached to the surface using 2bromo-2-methylpropionyl bromide by a one-step replacement reaction. The NIPAM solution mixed with PMDETA, $\mathrm{CuBr}$ and $\mathrm{CuBr}_{2}$ was degassed and injected into a vial containing pieces of $\mathrm{Si}$ wafer substrates to conduct the ATRP polymerization at room temperature. Finally, specimens featuring the polymer brush were soaked in EDTA solution overnight to remove the copper, and dried with nitrogen. More details on the sample preparation can be found in Ref. ${ }^{23}$ For a movie showing the sample preparation procedure see Ref. ${ }^{24}$ The grafting density and molar mass of the PNIPAM brush were estimated by the swelling ratio ${ }^{3 b}$ For the specimens used the values of $0.45 \pm 0.2$ chains $/ \mathrm{nm}^{2}$ and $2.5 \pm 1.0 \mathrm{x}$ $10^{5} \mathrm{~g} / \mathrm{mol}$ were obtained, respectively. The absolute molar mass of PNIPAM brushpolymers from reference samples with a dry height of $120 \mathrm{~nm}$ was determined to be $M_{n}=1$ $\mathrm{x} 10^{5} \mathrm{~g} / \mathrm{mol}$ (typical PDI $=4$ for the detached chains) ${ }^{25}$ by gel permeation chromatography in THF after detachment from the silicon substrate using $p$-toluene sulfonic acid. ${ }^{26}$

Static contact angle measurements were carried out on an optical contact angle device equipped with an electronic syringe unit (OCA15, Dataphysics, Germany). Contact angle values of the modified substrates at each reaction step were measured, and at least three samples were assessed for each step.

Fourier transform infrared spectroscopy (FTIR) was employed to characterize the chemical composition of the polymer brush tethered on the silicon substrates. A Bruker Vertex 70v spectrometer was used to obtain the FTIR spectra with a spectral resolution of $4 \mathrm{~cm}^{-1}$. Background data was obtained by scanning a clean silicon wafer before sample measurement.

Swelling and co-non-solvency properties were investigated by measuring brush thickness using a Variable Angle Spectroscopic Ellipsometer (VASE, J. A. Woollam). Measurements were performed as a function of photon energy (in ambient: $0.8-4.0 \mathrm{eV}$, corresponding to a wavelength range of 276-1550 nm; in ethanol-water mixture: 1.1-4 eV, e.g. 276-1127 nm) with a step size of $0.1 \mathrm{eV}$. In liquid, the accessible range is limited due to absorption of infrared light. The experiments were performed at room temperature $\left(25^{\circ} \mathrm{C}\right)$ in air and in different compositions of ethanol/water mixtures, as outlined in the materials and methods 
part. A dedicated custom-built liquid cell was used, which enables optical access at a fixed angle of incidence of $63^{\circ}$. Spectra on dry samples were performed at three different angles of incidence $\left(65,70\right.$ and $\left.75^{\circ}\right)$. The detailed analysis of the spectra is shown in appendix.

AFM force measurements were conducted on a Multimode 8 AFM with a NanoScope V controller, a JV vertical engage scanner and a liquid cell (Bruker, San Barbara, CA). Polystyrene (PS) colloids attached to soft silicon cantilevers (6 $\mu$ m diameter, CP-CONT-PS, sQube, NanoAndMore, Germany) were employed to determine the brush height at various ethanol-water compositions (typically $k=0.23 \mathrm{~N} / \mathrm{m} \pm 0.03$ ). Cantilevers with gold colloids (6 $\mu \mathrm{m}$ diameter, CP-FM-Au, sQube, NanoAndMore, Germany) were used to obtain the force curves and friction data. The deflection sensitivity and spring constant values (typical spring constants were in the range of $k=2.42 \mathrm{~N} / \mathrm{m} \pm 0.08$ ) of the cantilevers featuring gold colloid probes were determined in liquid for each composition used. The friction was measured by sliding the gold colloid probe over the brush in the lateral direction (over 20 $\mu \mathrm{m}$ at a velocity of $10 \mu \mathrm{m} / \mathrm{s}$ ), while measuring the torsional response of the cantilever. The torsional conversion factor (typically $S_{\theta}=1.42 \times 10^{-7} \mathrm{~N} / \mathrm{V}$ ) was calibrated by using Vezenov's noncontact method. ${ }^{27}$ The measurements were repeated on 3 different positions on the sample, where we found the same forces within 10\%. Experiments were repeated on 3 different samples that were prepared in the same batch and 3 more samples prepared in a different batch. For all these samples we found qualitatively the same results (15\% deviations in swelling ratios, $25 \%$ in the friction and 50\% in the adhesion (hysteresis)).

\subsection{Results and Discussion}

Figure 4.1 shows in (a) a schematic representation of the effect of the solvent composition on the measured brush height and in (b) the measured swelling ratio of PNIPAM brushes as a function of ethanol-water mixture at various compositions. In the experiments, the brush height is measured by ellipsometry (triangles up and down) and by determining the vertical distance between brush-surface and the bare silicon surface using AFM (squares and circles), respectively. We cannot expect the swollen heights measured by the different techniques to be the same, since there is no well-defined height of a brush. Instead, the polymer-density gradually changes as a function of the distance from the substrate ${ }^{6}$ and, as discussed in more detail below, the different measurement techniques different parts of this density distribution.

For the AFM measurements, we gently scratch off part of the brush by Teflon tweezers. Next, we inject the solvent and equilibrate the system for at least 10 minutes. Then, we determine the swollen height of the brush by (i) imaging under a small normal load of 1.6 $\mathrm{nN}$, (ii) performing force distance measurements $(\mathrm{Fd})$ upon approach of the colloid towards the brush. In the latter, we compare the (still arbitrary) separation where the force starts to deviate from 0 to the height of the brush imaged at a known applied force. The difference in 
tip-surface separation between the two is added to the brush height. The swollen height is normalized by dry brush height (close to $140 \mathrm{~nm}$ for the measurements shown in Figure $4.1)^{1}$ to give the swelling ratio shown in Figure 4.1. (b). We expect that we underestimate the brush height for the imaging method, as the brush is slightly compressed even under the lightest load applied. For the force distance measurements, we probably overestimate the brush height, because the force will increase as soon as the polymer density is high enough to bear a normal load, which can happen at larger distances than the brush height due to poly-dispersity. ${ }^{28}$ The difference in height for both measurements is most pronounced under good solvent conditions, where the brush stretches the most. This can be observed in pure ethanol, where the magnitude of the difference can be up to $250 \mathrm{~nm}$. The 'real' brush height will probably be between heights measured using imaging and the Fd method.

Figure 4.1. (b) also shows the swelling ratio measured with ellipsometry. A detailed explanation of the ellipsometry measurements can be found in the Appendix. In short, for the dry height measurements, the ellipsometry data is fitted to the traditional Cauchy model. For the swollen brush height measurements, we incorporate a gradual change in polymer density as a function of the distance from the surface. Such a gradual change is typically considered to be parabolic in the limit of dilute brushes ${ }^{29}$ but approaches a step function for high density brushes ${ }^{30}$ and can become linear or even concave in the presence of polydispersity. ${ }^{31}$ Since we do not know the exact density profile of our brushes, we tested two different models to fit the ellipsometry data. Model 1 (SGL) effectively fits the refractive index - and thus the density - to a power law function that can be tuned between concave and convex by changing the exponent. ${ }^{32}$ Model 2 (DL), places this gradually changing refractive index on top of a layer of constant refractive index (density) close to the sample surface. ${ }^{8}$ Both models give comparable results, except in pure water where the single gradient layer seems to underestimate the swollen height by a factor 1.4 and at 60 $70 \%$ ethanol where the double layer model overestimates the swollen height by a factor 1.31.8. For each individual measurement (AFM or ellipsometry), the new solvent-composition is applied to a cleaned and dried brush surface (e.g. the solvent composition is not continuously varied). We tested that our results were independent of previous solvent conditions by stepwise decreasing the vol.\% ethanol after stepwise increasing it. Moreover, we found the same swelling ratios for all the tested samples within $15 \%$.

\footnotetext{
${ }^{1}$ Please note that the measurements on Figure 4.1 were performed on different samples than the sample used for GPC and therefore the dry height is also different.
} 


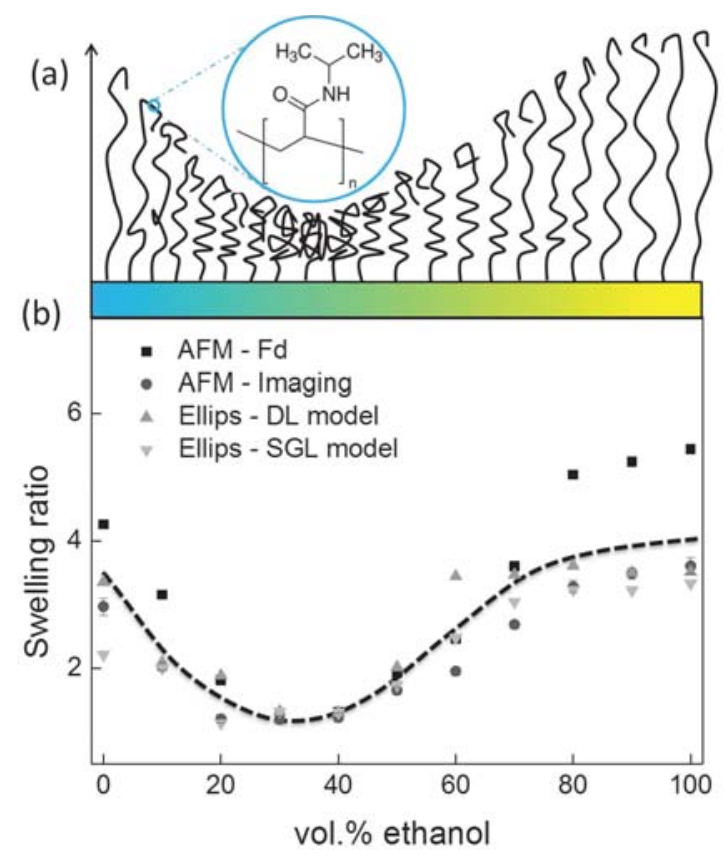

Figure 4.1. Schematic of the co-non-solvency-induced conformational change of the PNIPAM brush (dry height $140 \mathrm{~nm}$ ) (a) and the swelling ratio of a PNIPAM brush as a function of the ethanol volume fraction in the ethanol-water mixture. (b) The swelling ratio is calculated from the swollen height of the brushes and is normalized by the dry brush height as measured by AFM force distance (Fd, black squares) or imaging (dark gray circles) using a normal load that is kept as small as possible $(1.6 \mathrm{nN})$ over a scan size of 40 $\mu \mathrm{m}$ at a scan rate of $1 \mathrm{~Hz}$ or by ellipsometry measurements using either the double layer model (DL, gray upward triangles) or the single gradient layer model (SGL, light gray downward triangles).

In pure water, we find that the swelling ratio is about 3 , which is in agreement with the literature values reported for PNIPAM brushes with similar grafting densities ${ }^{33}$. When adding a small amount of ethanol to the water (10 vol. \% ethanol in the ethanol-water mixture), the PNIPAM brush strongly shrinks to reach a swelling ratio of around 2. By further increasing the ethanol volume fraction, the brush further collapses. At $30 \%$ ethanol volume fraction, there is a minimum in the swelling ratio. Here the swelling ratio has, however, still a value of 1.25 . The observation that the system is still partly solvated at minimum swelling is in agreement with results obtained by others. Dalkas et al. ${ }^{34}$ measured the cloud point of PNIPAM in water-dioxane mixtures, and the point of minimum swelling is obtained around $30-40 \%$ dioxane volume fraction. Napper et al. ${ }^{17}$ find for PNIPAM on polystyrene latex particles that the minimum particle-diameter is obtained at $30 \%$ ethanol volume fraction, and the swelling ratio is calculated to be roughly 1.5 . This is in direct agreement with our measurements. The presence of solvent in the collapsed brush is consistent with the bridging-model of Mukherji et al., ${ }^{18 \mathrm{~b}}$ where the better solvent is expected to be between the collapsed polymers. However, this remains speculative, since we cannot tell from our results the partition of water and ethanol within the brush. We note 
that the presence of solvent in a collapsed brush is not observed for a temperature-induced conformational change of a PNIPAM brush. ${ }^{23,35}$ For these systems, the swelling ratio has been found to be close to 1 above the lower critical solution temperature (LCST). This might indicate that the mechanism of collapse is different for a temperature-induced collapse compared to a co-non-solvency-induced collapse.

At a $40 \%$ ethanol volume fraction, the average swelling ratio is 1.3 and is slightly higher than at $30 \%$, which shows that the brush again absorbs more solvent molecules and reswells. This is called the re-entrant phenomenon. ${ }^{18 a}$, 34, 36 By further increasing the ethanol content, the brush swells more until it reaches its maximum swelling ratio in $100 \%$ ethanol. In pure ethanol, the swelling ratio is larger than 3.5, which is close to the height expected for fully stretched polymers and is more than $20 \%$ times higher than in pure water. This higher swelling ratio shows that ethanol is a better solvent for PNIPAM than water.

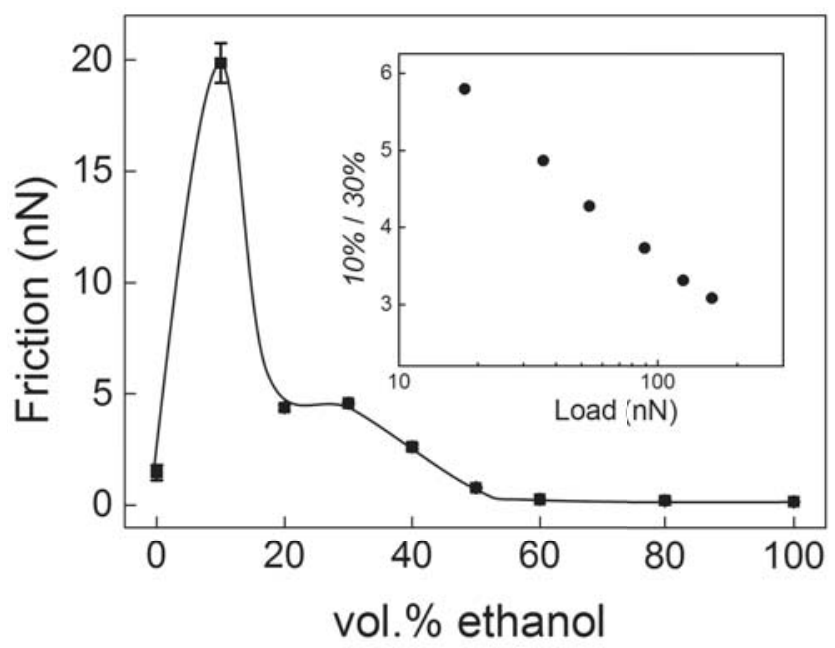

Figure 4.2. Friction force between the PNIPAM brush (dry height $170 \mathrm{~nm}$ ) and a gold colloid as a function of ethanol volume fraction measured at a relative velocity of $10 \mu \mathrm{m} / \mathrm{s}$ under a normal load of $25 \mathrm{nN}$, while moving over a scan size of $20 \mu \mathrm{m}$. The error bars denote the standard error of the mean with a $95 \%$ confidence interval. For most data points the error bars are smaller than the symbol size. The inset shows the friction at $10 \%$ ethanol normalized by the friction at $30 \%$ ethanol between a PS probe (radius $7 \mu \mathrm{m}$ ) and the PNIPAM brush (dry height $115 \mathrm{~nm}$ ) upon sliding at a velocity of $20 \mu \mathrm{m} / \mathrm{s}$ over a scan size of $20 \mu \mathrm{m}$.

So far, most studies on PNIPAM have focused on investigating the change in chemical or physical properties by comparing the system for two states, the swollen state and the collapsed state. ${ }^{8,} 37$ In our recent publication, ${ }^{23}$ we show, however, that the tribo-mechanical properties of PNIPAM brushes change the strongest at the high temperature range of the coil-to-globule transition. In Ref., ${ }^{23}$ we systematically vary the temperature and find that the friction just below the LCST is almost two orders of magnitude higher than the friction at 
lower or higher temperatures. Therefore, we expect that close to the coil-to-globule and globule-to-coil transition of PNIPAM in water ethanol mixtures, the friction will be high too.

Figure 4.2 shows the variation of friction force between the PNIPAM brush and a gold colloid as a function of the volume fraction of ethanol in the ethanol-water mixture. In water, the PNIPAM brush is swollen (see Figure 4.1) and the friction force is only $1.5 \mathrm{nN}$ (Figure 4.2). When the brush is water-swollen, it is very lubricious. However, when a small amount of ethanol $(10 \%)$ is added, the friction force dramatically increases to about $21 \mathrm{nN}$, which is 14 times higher than the value observed in water. Such a strong variation of friction is also found for PNIPAM brushes in water close to the LCST. ${ }^{23}$ As shown in Figure 4.1 for a $10 \%$ ethanol-water mixture, the PNIPAM brush height decreases by $36 \%$ compared to the height in water and, thus, the polymer density is higher. This allows for forming more interaction-sites between the gold colloid and the PNIPAM brush. When sliding over the partially collapsed brush, the colloid can stretch the chains in the brush, which would enhance friction. A schematic representation of this postulated effect is shown in Figure 4.3. At the given solvent composition, the friction increases logarithmically with the velocity, which is consistent with the observations in recent molecular dynamics simulations using attractive polymer-surface interactions. ${ }^{38}$ In these simulations, it was shown that the dissipation upon sliding is caused by the formation and rupture of physical polymer-surface bonds, which causes instabilities and, thus yields a logarithmic dependence of the friction on the velocity.

Upon further increase of the ethanol content to a volume fraction of $20 \%$, the PNIPAM brush-height shrinks by $40 \%$. However, the friction now decreases. The reason for this might be that the more strongly collapsed brush at $20 \%$ ethanol content resists stretching thus reduce the related energy dissipation, yielding a lower friction. The maximum collapse (minimum swelling ratio in Figure 4.1) occurs at 0.3 ethanol-fraction. In the maximally collapsed state, the friction is a $1.05 \pm 0.03$ times higher compared to the friction at $20 \%$. The mostly collapsed brush resists to be stretched due to the increased polymer-polymer interactions, and the friction is now mainly dominated by adherence interactions and viscoelastic deformations while sliding over the rough collapsed brush. Thus, a second, but smaller, maximum of friction is observed at this concentration.

When the ethanol volume fraction is further increased to $50 \%$ ethanol, the brush reswells. An effective lubricant layer is formed again and, as a result, the friction decreases to $0.8 \mathrm{nN}$. Chen et al. ${ }^{8}$ report that in a 50/50 (v/v) methanol/water solvent-co-solvent mixture, friction force between the PNIPAM brush and silicon colloid is 4.6 times than in water. In contrast, we find that in a $50 \%$ ethanol-water mixture the friction is lower than that in water. A possible explanation for this discrepancy might be that we measured at different temperatures. The solvation of PNIPAM brushes is very sensitive to the temperature, such that even a few degrees difference in temperature can alter the friction by orders of 
magnitude. ${ }^{23}$ Surprisingly, even though we have a re-entrance or globule-to-coil transition at $50 \%$ ethanol, we do not observe a strong response in the friction. This asymmetry might indicate that the mechanism causing the coil-to-globule transition at $10 \%$ ethanol is different from the mechanism behind the globule-to-coil transition at 50\% ethanol in the solvent mixture, which would be consistent with the bridging-by-the-better-solvent picture of Mukherji et al. ${ }^{18 \mathrm{~b}}$ However, an alternative explanation could be that the presence of ethanol disrupts the PNIPAM-gold interactions.

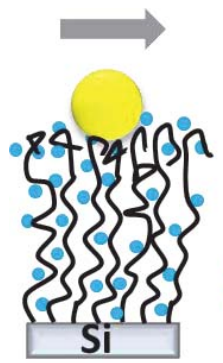

water

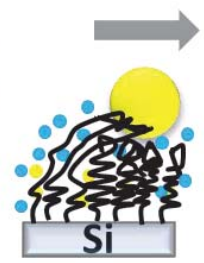

$10 \%$

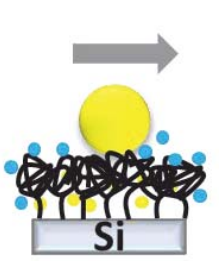

$30 \%$

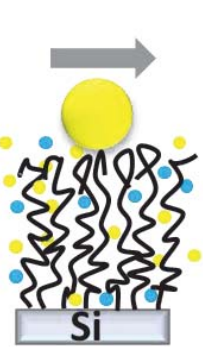

$50 \%$

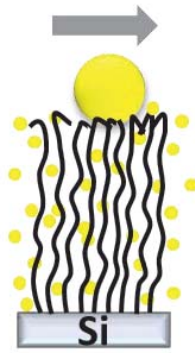

ethanol

Figure 4.3. Schematic illustration of the proposed origin of friction between the gold colloid and the PNIPAM brush at various compositions of water (blue) and ethanol (yellow).

Above $60 \%$ ethanol in the ethanol-water mixture, the friction is almost constant at a low value of $0.2 \mathrm{nN}$. Because alcohol is a better solvent for PNIPAM than water, ${ }^{10}, 39$ the PNIPAM brushes will preferentially interact with ethanol molecules instead of gold. Moreover, the high osmotic pressure in the ethanol also reduces the contact area between colloid and brush. Thus, very low friction forces can be obtained. In pure ethanol, the friction force is as low as $0.16 \mathrm{nN}$, which is two orders of magnitude smaller than the friction in $10 \%$ ethanol-water mixture. In pure ethanol, we find that the friction increases linearly with the velocity, which implies that the dissipation is dominated by hydrodynamic drag and not by polymer-colloid interactions.

The observed variation in the friction is strongest at low normal load values, as depicted in the inset of Figure 4.2. Upon increasing the normal load, the ratio of the friction force at $10 \%$ ethanol compared to the friction at $30 \%$ decreases logarithmically with the load. In the limit of very high loads, the friction will become independent of the solvent composition. It is, however, difficult to say at what load this will happen, since different dissipation mechanisms would dominate the friction at high normal loads.

Within a $15 \%$ uncertainty, we observe the same changes in friction as shown in Figure 4.2 in measurements on 6 different samples using 4 different gold colloid probes. When a polystyrene colloid probe (colloid diameter $6 \mu \mathrm{m}$ ) is employed for the experiments, the 
variation in friction force reduces to a factor $72-95$ (with variations around $25 \%$ ). This observation implies that the change in friction force depends on the solvent-mediated interaction between the colloid and the PNIPAM brush.

To study if polymer stretching could be at the origin of the enhanced frictional response at $10 \%$ ethanol content in the ethanol-water mixture, we performed AFM experiments in which we measured the force on the colloid probe upon approach to and retract from the brush surface (displacement of $2 \mu \mathrm{m}$ at a velocity of $10 \mu \mathrm{m} / \mathrm{s}$ ). Figure 4.4 shows typical raw force-separation curves of a PNIPAM brush in various compositions of the ethanol-water mixture. The maximum compression load was controlled to be $80 \mathrm{nN}$. As expected, in pure water we observe almost no adhesive interaction. When the brush is kept in a $10 \%$ ethanolwater mixture, the adhesive interaction is clearly stronger compared to what we found in pure water. The maximum adhesion force at $10 \%$ ethanol is $6 \mathrm{nN}$, which is 3.4 times larger than the maximum adherence in water. Also, there is a pronounced long-ranged adhesive interaction that vanishes only at distances larger than $1.32 \pm 0.11 \mu \mathrm{m}$. As shown in our previous work on the adhesive interactions between PNIPAM and a gold colloid upon inducing a swelling transition using temperature, ${ }^{23}$ we attribute this long-ranged adhesive interaction to stretching of the partly collapsed polymers. Similar stretching of polymers has been observed in Ref. ${ }^{40}$ Upon moving the colloid in the lateral direction, this stretching of the brush-polymers can increase friction (shown in Figure 4.2). Stretching over such a large distance seems surprising considering our degree of polymerization, which is typically smaller than 2200. However, our polydispersity index is high (PDI is 3-5), such that the longer chains in the brush can still stretch over distances around $1.3 \mu \mathrm{m}$. We note that such a high polydispersity index could indicate cross-linking in the brush. However, crosslinking would counteract polymer-stretching as is, therefore, probably not the main cause for our high PDI. As shown in the Appendix 4.4, thinner brushes (20 nm dry height) show significantly less polymer stretching over only $650 \mathrm{~nm}$.

For an ethanol volume fraction of $30 \%$, the brush height is the smallest. At this concentration, the retract curve shows a sharp snap-off instability and a maximum adherence of $110 \mathrm{nN}$. No long-ranged adhesion is observed and thus chains would not become stretched. At a 50\% ethanol volume fraction, the water-ethanol mixture solvates the brush such that the swelling ratio is 1.8. The sharp snap-off instability and adherence (40 $\mathrm{nN}$ ) is less strong than for $30 \%$ ethanol. In pure ethanol, the snap-off instability has completely vanished and we observe no significant adhesion interaction.

Even though the swelling ratio is still 1.5 at maximum collapse of the brush at $30 \mathrm{vol} \%$ of ethanol in water, we observe force curves that exhibit a sharp snap-off instability, which is typically only observed for fully collapsed brush. However, we note that in our experiments we probe the adherence between the colloid and the top of the brush. Therefore, we would like to argue that our results imply that the brush is collapsed at the top for ethanol volume 
fractions between $20-60 \%$, which would be in agreement with the observation of Chen et $a .^{8}$

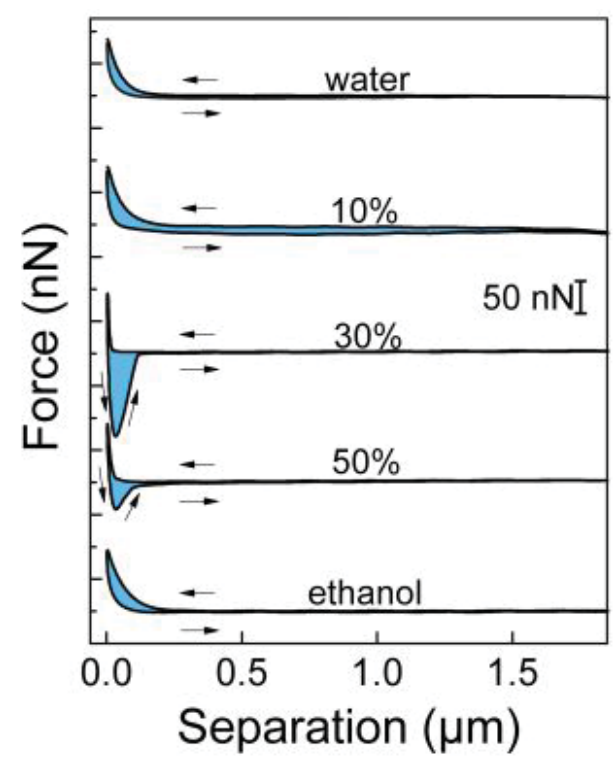

Figure 4.4. Typical force versus separation curves at various ethanol volume fractions ( 0 , $10,30,50,100 \%)$. The approach and retract velocity of the gold colloid to and from the PNIPAM brush (dry height $170 \mathrm{~nm}$ ) is $10 \mu \mathrm{m} / \mathrm{s}$. The arrows denote the direction of motion. The difference in force between the approach and retract curves is colored blue to clarify the adhesive interaction. The scale bar equals $50 \mathrm{nN}$.

\subsection{Conclusions}

In summary, we have shown that tunable friction is provided by co-non-solvency of a PNIPAM brush in ethanol-water mixtures. In pure water, the PNIPAM brush is swollen and acts as a lubricant layer. For $10 \%$ ethanol in the ethanol-water mixture, the PNIPAM brush is partially collapsed and a large friction force is observed due to stretching of the partly collapsed brush chains when the colloid probe is moved away from the initial point of contact. The strongest collapse of the PNIPAM brush occurs in 30\% volume fraction of ethanol. As expected, when the brush is in this state, the friction is higher than in pure water. Its value is, however, smaller than at $10 \%$ ethanol-content, since the grafted chains do not stretch in this case during the sliding motion. At higher volume fractions of ethanol, the brush re-swells. At these solvent compositions, a lubricant layer is again formed. The friction in this case gradually decreases with increasing ethanol-content and reaches a minimum in pure ethanol. At this minimum, typical values of the friction force are two orders of magnitude smaller than the forces measured at $10 \%$ ethanol in the ethanol-water mixture. Our results show that friction can be tuned in a straightforward manner by varying the relative amount of ethanol. Such tunable friction by employing co-non-solvency of PNIPAM brush in ethanol-water mixtures has potential application in walking robots, and switchable tweezers. 


\subsection{Appendix}

\section{Analysis of ellipsometry spectra}

For the determination of the optical constants and film thickness from ellipsometry spectra one needs to establish an optical model, which enables describing the experimental data in terms of actual physical parameters. The optical dispersion $(n)$ can be modelled with the Cauchy dispersion model

$n(\lambda)=A+B / \lambda^{2}+C / \lambda^{4}$

where $n$ is the refractive index, and $A, B$ and $C$ are the Cauchy parameters which may be obtained from the model to the data; $\lambda$ is the wavelength in micrometers. The polymer film is considered to be a transparent "dielectric", with the assumption that the absorption coefficient is close to zero $(k=0)$ and consequently, the refractive index is a real quantity.

\section{Silicon substrate and ambient}

The optical properties of the substrate, consisting of bulk silicon with $2.0 \mathrm{~nm}$ of a native oxide, and the solvents (water and ethanol) were used as available in the CompleteEASE package (the refractive index at $600 \mathrm{~nm}$ of pure water and pure ethanol are 1.33 and 1.36 at $20^{\circ} \mathrm{C}$, respectively). The refractive indexes of the various ethanol/water mixtures were obtained by considering them as sum of the product between the volume fraction and the pure refractive index of the single components; the extinction coefficients were considered to be zero.

\section{Dry brush films}

The experimental data obtained on dry films in air were fitted between 250-1150 nm with a model consisting of a single uniform Cauchy layer (representing the PNIPAM film) on top of the silicon substrate. The thickness $d$, and the Cauchy parameters $A$ and $B$ were used as fitting parameters; as the $C$ Cauchy parameter does not yield improved fitting results, it was set to 0 .

The PNIPAM film was considered to be uniform, and the roughness of the polymeric layer was neglected; the material density is distributed evenly within the volume of film and consequently there was no gradient in the optical refractive index.

\section{Wet brush films}

In liquid, the experimental data were fitted between 276-1127 nm with three different uniform layer models, each assuming the same silicon substrate (with native silicon oxide layer). The three models are schematically represented in Appendix 4.1 and details are summarized below:

1. a dense Cauchy layer model, in which thickness $d$, and Cauchy parameters A and B were varied; the Cauchy parameter $\mathrm{C}$ was set to 0 . 
2. a single gradient layer; the gradient in the optical density is described by an exponential decrease of $\mathrm{A}$, as a function of the distance from the substrate (layer composed of 50 slices). Only the A-parameter was considered, mainly for two reasons: in the Cauchy model of eq.1, the A parameter is the principal contributor of the refractive index and secondly, keeping the number of fitting parameters limited simplifies the analysis and the extraction of physical information. The thickness $d$, the Cauchy parameters $A$ and $B$, the percentage variation of the A-parameter $(\% A)$ and the exponent of the exponential equation for the decrease of A-parameter (exp) were fitted to the experimental data; the C Cauchy parameter was set to 0 .

3. a double layer model; On top of the silicon substrate, a dense uniform layer (represented by a thickness $\mathrm{d} 1$ and Cauchy parameters A and B) is followed by a second diluted layer with a gradient of the A parameter. The latter is represented by a linear decrease of the optical density as a function of the distance from the substrate (layer composed of 50 slices). The outermost linear gradient layer was modelled by the thickness $d_{2}$, Cauchy parameters $A$ the $B$ and the optical density variation $\% A$. For both layers, the Cauchy parameter was set to 0 .

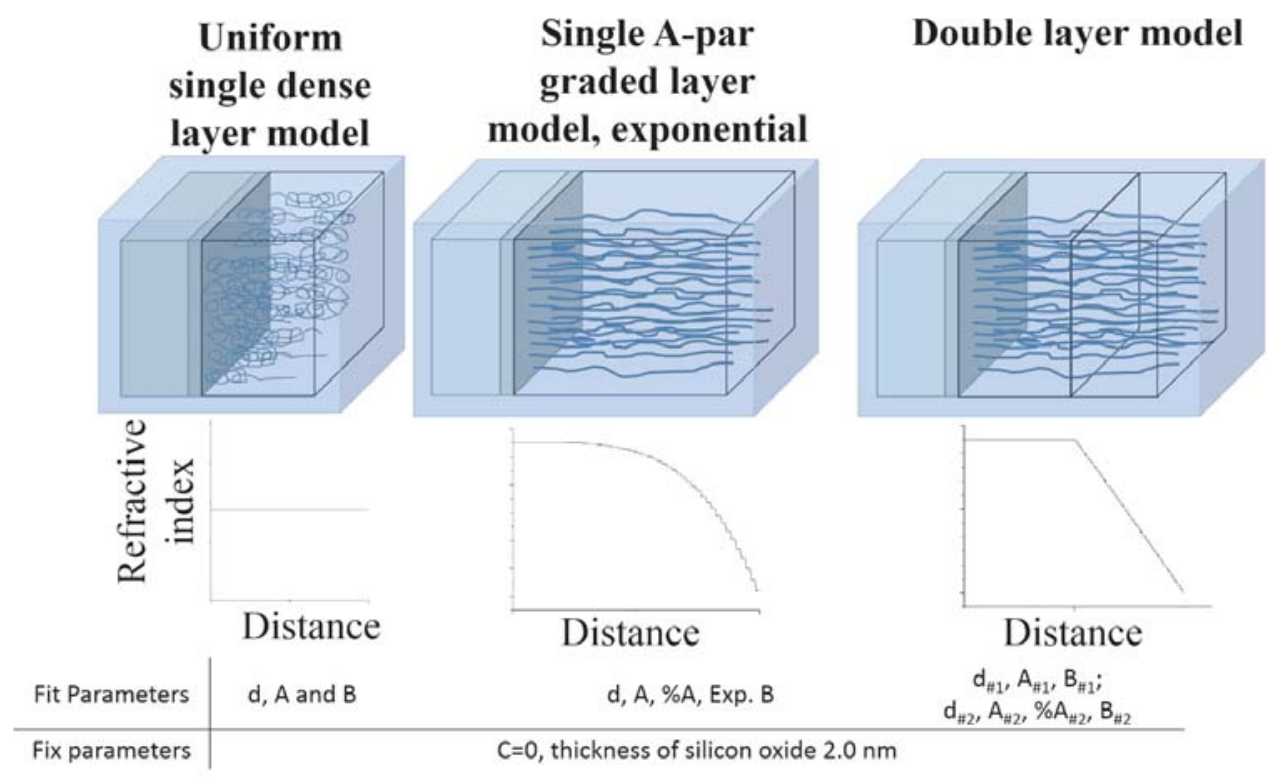

Appendix 4.1. Schematic representation of the ellipsometry models used for describing the optical properties of the polymer brushes in water 


\section{Comparison between swollen in water and in ethanol}

Upon immersion of the PNIPAM brush film, swelling is observed, as shown in Appendix 4.2. The swelling behavior is different depending on whether the polymeric layer is immersed in pure water or in pure ethanol. Consequently, the change of the optical parameters for each composition of medium (ethanol-water mixtures) were considered.
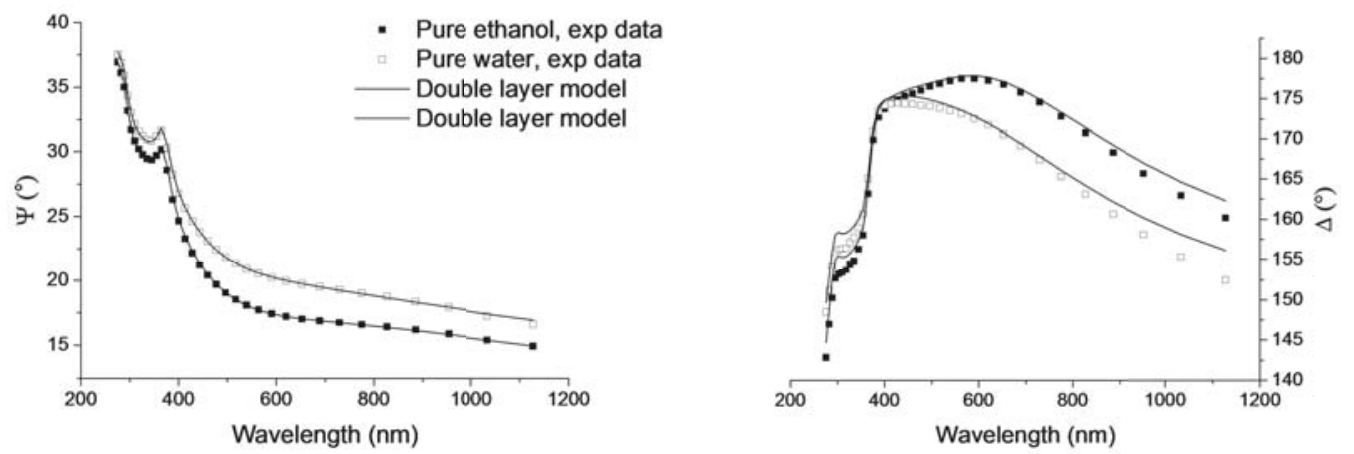

Appendix 4.2. In-situ ellipsometry spectra (symbols) and fit results (solid lines) for PNIPAM brush grafted on silicon swollen in water (open symbols) and in ethanol (filled symbols). For the fitting of the experimental data was used the double layer model described in the support information.

For all experimental data, the curves were first fitted using the single uniform layer, subsequently the exponential single gradient layer, and finally the double Cauchy layer model.

Appendix 4.3 shows the dependence and the trend of the optical parameters on solvent composition at fixed room temperature. The change in $\Psi$ and $\Delta$ is evident between the volume ratio of 0.20 and 0.50 . The changes in $\Delta$ are more evident that the changes in $\Psi$. 

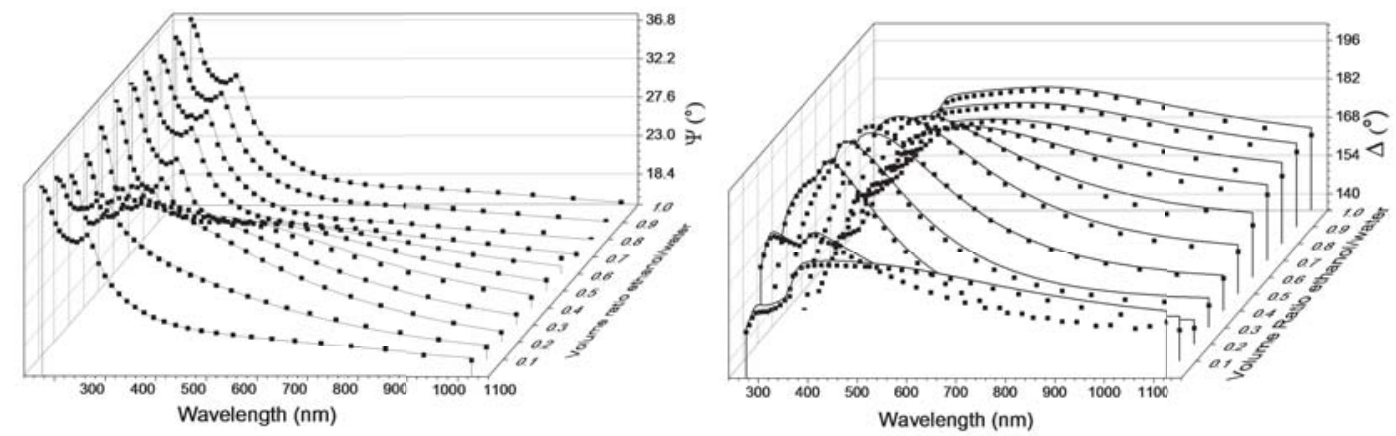

Appendix 4.3. Experimental ellipsometry spectra (symbols) and fit results (solid lines) for the in-situ PNIPAM films on silicon using the double layer model to describe the swollen state of the brushes between swollen in pure water and in volume ratio ethanol/water 0.1 .

Table 4.1. Fit parameters using a double layer model for the optical modelling of PNIPAM brush layer in solvency and co-non-solvency. The sample was immersed in different volume ratio of ethanol-water mixture at room temperature.

\begin{tabular}{|c|c|c|c|c|c|c|c|c|c|c|c|c|}
\hline & & $0 \%$ & $10 \%$ & $20 \%$ & $30 \%$ & $40 \%$ & $50 \%$ & $60 \%$ & $70 \%$ & $80 \%$ & $90 \%$ & $100 \%$ \\
\hline \multicolumn{2}{|c|}{$\begin{array}{c}\text { TOT } \\
\text { thickness } \\
(\mathrm{nm})\end{array}$} & 396 & 247 & 222 & 156 & 154 & 238 & 406 & 409 & 426 & 413 & 414 \\
\hline \multirow{5}{*}{ 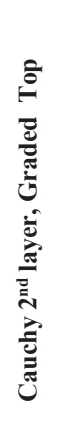 } & $\begin{array}{c}\mathrm{d}_{2} \\
(\mathrm{~nm})\end{array}$ & 271 & 151 & 160 & - & - & 183 & 236 & 255 & 282 & 270 & 275 \\
\hline & A & 1,312 & 1,360 & 1,405 & - & - & 1,415 & 1,370 & 1,372 & 1,372 & 1,373 & 1,371 \\
\hline & $\begin{array}{c}\mathrm{B} \\
\left(\mu \mathrm{m}^{2}\right. \\
)\end{array}$ & 0,00173 & $\stackrel{-}{-}$ & 0,00259 & - & - & 0,00486 & 0,00115 & 0,00244 & 0,00257 & 0,00224 & 0,00232 \\
\hline & $\% \mathrm{~A}$ & $-2,42$ & $-5,73$ & $-13,26$ & - & - & $-1,86$ & $-3,15$ & $-2,61$ & $-2,17$ & $-2,04$ & $-2,04$ \\
\hline & Exp & 1 & 1 & 1 & - & - & 1 & 1 & 1 & 1 & 1 & 1 \\
\hline \multirow{3}{*}{ 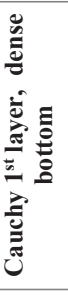 } & $\begin{array}{c}\mathrm{d}_{1} \\
(\mathrm{~nm})\end{array}$ & 125 & 96 & 62 & 156 & 154 & 33 & 170 & 154 & 144 & 143 & 139 \\
\hline & $\mathrm{A}$ & 1,395 & 1,408 & 1,435 & 1,447 & 1,452 & 1,423 & 1,411 & 1,401 & 1,403 & 1,402 & 1,401 \\
\hline & $\begin{array}{c}\mathrm{B} \\
\left(\mu \mathrm{m}^{2}\right. \\
)\end{array}$ & 0,00176 & 0,00284 & 0,00259 & 0,00393 & 0,00410 & 0,00092177 & 0,00356 & 0,00401 & 0,00271 & 0,00287 & 0,00252 \\
\hline \multicolumn{2}{|c|}{ Ambient } & $0 \%$ & $10 \%$ & $20 \%$ & $30 \%$ & $40 \%$ & $50 \%$ & $60 \%$ & $70 \%$ & $80 \%$ & $90 \%$ & $100 \%$ \\
\hline
\end{tabular}

In general, in the case of swollen PNIPAM brush films the double layer model provided the best description of the experimental data. This phenomena typically occurs when the 
PNIPAM film is immersed in solvent mixtures with volume ratio ethanol/water below 0.1 and above 0.6.

In the other hand, the PNIPAM collapsed when the composition of the ethanol-water mixtures is between 0.2 and 0.5 . In this case, the dense uniform single layer model resulted in an adequate description of the experimental data. The fit parameter obtained with the double layer models are summarized respectively in the Table 4.1.

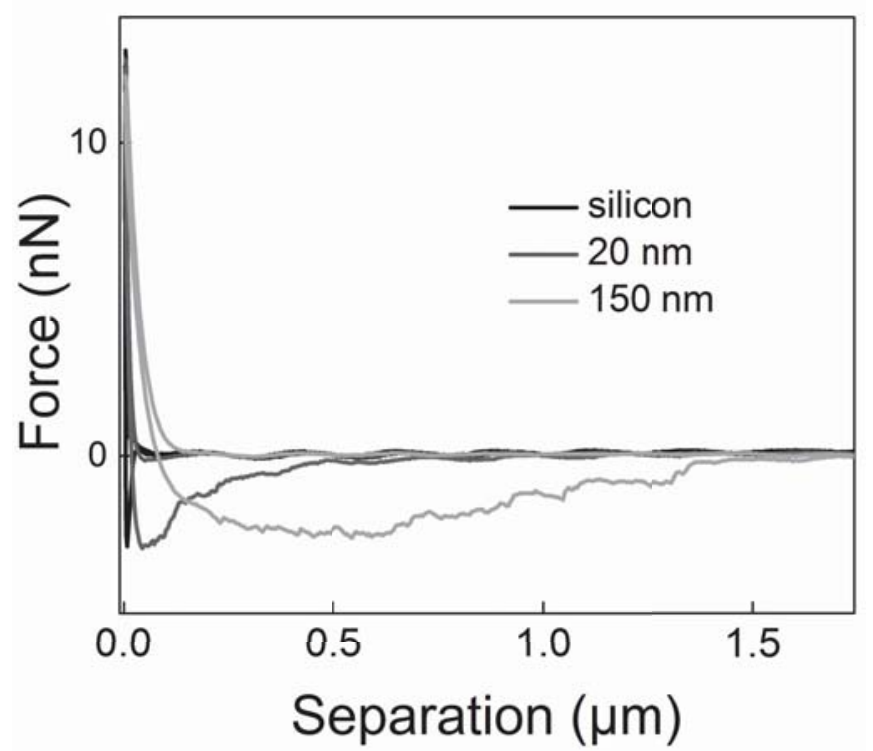

Appendix 4.4. Force-Separation curve of bare silicon substrate, $20 \mathrm{~nm}$ PNIPAM and 150 nm PNIPAM brush in 10\% ethanol volume fraction in water.

\subsection{References}

[1] A. J. Ijspeert, Science 2014, 346, 196-203.

[2] Y. Wu, M. R. Cai, X. W. Pei, Y. M. Liang and F. Zhou, Macromolecular Rapid Communications 2013, 34 , 1785-1790.

[3] a) T. L. Sun, G. J. Wang, L. Feng, B. Q. Liu, Y. M. Ma, L. Jiang and D. B. Zhu, Angewandte ChemieInternational Edition 2004, 43, 357-360; b) I. B. Malham and L. Bureau, Langmuir 2010, 26, 4762-4768; c) D. M. Jones, J. R. Smith, W. T. S. Huck and C. Alexander, Advanced Materials 2002, 14, 1130-1134; d) E. M. Benetti, S. Zapotoczny and J. Vancso, Advanced Materials 2007, 19, 268-271.

[4] a) X. F. Sui, Q. Chen, M. A. Hempenius and G. J. Vancso, Small 2011, 7, 1440-1447; b) A. Li, S. N. Ramakrishna, P. C. Nalam, E. M. Benetti and N. D. Spencer, Advanced Materials Interfaces 2014, 1, 1300007; c) S. de Beer, E. Kutnyanszky, P. M. Schon, G. J. Vancso and M. H. Muser, Nature Communications 2014, 5, 3781.

[5] a) H. Lee, B. P. Lee and P. B. Messersmith, Nature 2007, 448, 338-342; b) J. P. Gong, Soft Matter 2006, 2 , 544-552.

[6] S. T. Milner, Science 1991, 251, 905-914.

[7] M. K. Vyas, K. Schneider, B. Nandan and M. Stamm, Soft Matter 2008, 4, 1024-1032.

[8] Q. Chen, E. S. Kooij, X. F. Sui, C. J. Padberg, M. A. Hempenius, P. M. Schon and G. J. Vancso, Soft Matter 2014, 10, 3134-3142.

[9] H. J. Dai, Q. Chen, H. L. Qin, Y. Guan, D. Y. Shen, Y. Q. Hua, Y. L. Tang and J. Xu, Macromolecules 2006, 39, 6584-6589.

[10] F. M. Winnik, H. Ringsdorf and J. Venzmer, Macromolecules 1990, 23, 2415-2416. 
[11] T. Wu, P. Gong, I. Szleifer, P. Vlcek, V. Subr and J. Genzer, Macromolecules 2007, 40, 8756-8764.

[12] R. Jakubiak, T. J. Bunning, R. A. Vaia, L. V. Natarajan and V. P. Tondiglia, Advanced Materials 2003, $15,241-244$.

[13] J. M. Shen, F. Y. Gao, L. P. Guan, W. Su, Y. J. Yang, Q. R. Li and Z. C. Jin, Rsc Advances 2014, 4, 18473-18484.

[14] a) Q. B. Wei, M. R. Cai, F. Zhou and W. M. Liu, Macromolecules 2013, 46, 9368-9379; b) Y. L. Yu, B. D. Kieviet, E. Kutnyanszky, G. J. Vancso and S. de Beer, Acs Macro Letters 2015, 4, 75-79; c) S. de Beer, Langmuir 2014, 30, 8085-8090.

[15] a) C. Scherzinger, P. Lindner, M. Keerl and W. Richtering, Macromolecules 2010, 43, 6829-6833; b) C. Scherzinger, A. Schwarz, A. Bardow, K. Leonhard and W. Richtering, Current Opinion in Colloid \& Interface Science 2014, 19, 84-94; c) F. Tanaka, T. Koga and F. M. Winnik, Physical review letters 2008, 101, 028302; d) H. Wang, Y. An, N. Huang, R. J. Ma and L. Q. Shi, Journal of Colloid and Interface Science 2008, 317, 637-642; e) F. M. Winnik, M. F. Ottaviani, S. H. Bossmann, W. Pan, M. Garcia-Garibay and N. J. Turro, Macromolecules 1993, 26, 4577-4585.

[16] a) D. Mukherji and K. Kremer, Macromolecules 2013, 46, 9158-9163; b) K. Mukae, M. Sakurai, S. Sawamura, K. Makino, S. W. Kim, I. Ueda and K. Shirahama, Journal of Physical Chemistry 1993, 97, $737-$ 741.

[17] P. W. Zhu and D. H. Napper, Journal of Colloid and Interface Science 1996, 177, 343-352.

[18] a) M. Z. Liu, F. L. Bian and F. L. Sheng, European Polymer Journal 2005, 41, 283-291; b) D. Mukherji, C. M. Marques and K. Kremer, Nature communications 2014, 5, 4882.

[19] D. Mukherji, C. M. Marques, T. Stuehn and K. Kremer, The Journal of chemical physics 2015, 142, 114903.

[20] a) T. H. Young and W. Y. Chuang, Journal of Membrane Science 2002, 210, 349-359; b) B. R. Saunders, H. M. Crowther and B. Vincent, Macromolecules 1997, 30, 482-487.

[21] Y. Ito, T. Ito, H. Takaba and S. Nakao, Journal of Membrane Science 2005, 261, 145-151.

[22] L. Liu, X. L. Song, X. J. Ju, R. Xie, Z. Liu and L. Y. Chu, Journal of Physical Chemistry B 2012, 116, 974-979.

[23] Y. L. Yu, B. D. Kieviet, F. Liu, I. Siretanu, E. Kutnyánszky, G. J. Vancso and S. de Beer, Soft Matter 2015, DOI: 10.1039/C1035SM01426C.

[24] S. de Beer, E. Kutnyanszky, M. H. Muser and G. J. Vancso, Jove-Journal of Visualized Experiments 2014, 52285.

[25] J. Pyun, T. Kowalewski and K. Matyjaszewski, Macromolecular Rapid Communications 2003, 24, 10431059.

[26] C. Cummings, H. Murata, R. Koepsel and A. J. Russell, Biomaterials 2013, 34, 7437-7443.

[27] K. Wagner, P. Cheng and D. Vezenov, Langmuir 2011, 27, 4635-4644.

[28] S. M. Balko, T. Kreer, P. J. Costanzo, T. E. Patten, A. Johner, T. L. Kuhl and C. M. Marques, Plos One 2013, 8 .

[29] a) A. N. Semenov, Zhurnal Eksperimentalnoi I Teoreticheskoi Fiziki 1985, 88, 1242-1256; b) S. T. Milner, T. A. Witten and M. E. Cates, Macromolecules 1988, 21, 2610-2619; c) A. M. Skvortsov, I. V. Pavlushkov, A. A. Gorbunov, Y. B. Zhulina, O. V. Borisov and V. A. Pryamitsyn, Polymer Science U.S.S.R. 1988, 30, 1706-1715.

[30] a) P. M. Biesheuvel, W. M. de Vos and V. M. Amoskov, Macromolecules 2008, 41, 6254-6259; b) D. F. K. Shim and M. E. Cates, Journal De Physique 1989, 50, 3535-3551.

[31] a) N. Dan and M. Tirrell, Macromolecules 1993, 26, 6467-6473; b) W. M. de Vos and F. A. M. Leermakers, Polymer 2009, 50, 305-316.

[32] E. S. Kooij, X. F. Sui, M. A. Hempenius, H. J. W. Zandvliet and G. J. Vancso, Journal of Physical Chemistry B 2012, 116, 9261-9268.

[33] a) E. Bittrich, S. Burkert, M. Muller, K. J. Eichhorn, M. Stamm and P. Uhlmann, Langmuir 2012, 28, 3439-3448; b) S. B. Rahane, J. A. Floyd, A. T. Metters and S. M. Kilbey, Advanced Functional Materials 2008, 18, 1232-1240.

[34] G. Dalkas, K. Pagonis and G. Bokias, Polymer 2006, 47, 243-248.

[35] J. Zhang, L. Y. Chu, Y. K. Li and Y. M. Lee, Polymer 2007, 48, 1718-1728.

[36] S. Y. Oh and Y. C. Bae, Polymer 2013, 54, 2308-2314.

[37] a) M. A. Cole, N. H. Voelcker, H. Thissen, R. G. Horn and H. J. Griesser, Soft Matter 2010, 6, $2657-$ 2667; b) Y. K. Jhon, R. R. Bhat, C. Jeong, O. J. Rojas, I. Szleifer and J. Genzer, Macromolecular rapid communications 2006, 27, 697-701; c) G. M. Liu and G. Z. Zhang, Journal of Physical Chemistry B 2005, 109, 743-747; d) S. H. Ma, D. A. Wang, Y. M. Liang, B. Q. Sun, S. N. Gorb and F. Zhou, Small 2015, 11, 1131-1137. 
[38] M. K. Singh, P. Ilg, R. M. Espinosa-Marzal, M. Kroger and N. D. Spencer, Langmuir 2015, 31, 47984805.

[39] T. Gilanyi, I. Varga, R. Meszaros, G. Filipcsei and M. Zrinyi, Physical Chemistry Chemical Physics 2000, 2, 1973-1977.

[40] a) A. Drechsler, A. Synytska, P. Uhlmann, M. M. Elmahdy, M. Stamm and F. Kremer, Langmuir 2010, 26, 6400-6410; b) J. D. Willott, T. J. Murdoch, G. B. Webber and E. J. Wanless, Macromolecules 2016, 49, 2327-2338. 



\section{Chapter 5}

\section{Pick up, Move and Release of Nanoparticles Utilizing Co-non-solvency of PNIPAM Brushes}

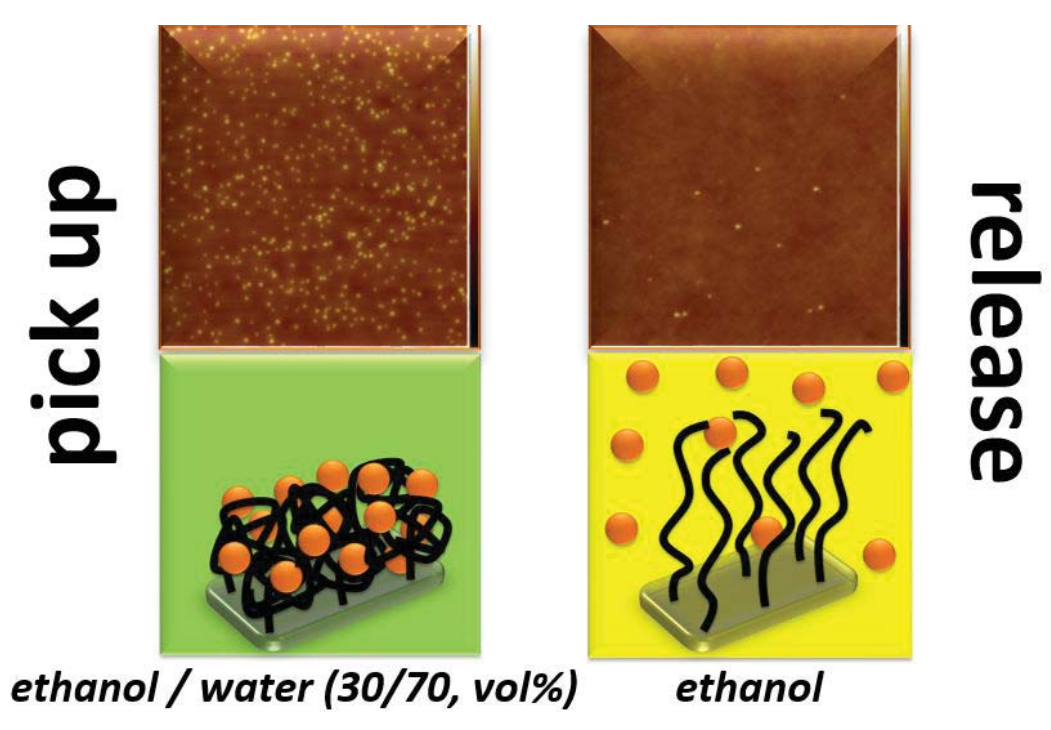

A critical complication in handling nanoparticles is the formation of aggregates when particles are dried e.g. for transfer from one liquid to another. The particles in these aggregates need to disperse into the destined liquid medium, which has been proven difficult due to the relatively large interfacial interactions between nanoparticles. We present a simple method to capture, move and release nanoparticles without the formation of large aggregates. To do so, we employ the co-non-solvency effect of $\operatorname{poly}(\mathrm{N}-$ isopropylacrylamide) (PNIPAM) brushes in water-ethanol mixtures. In pure water or ethanol, the densely end-anchored macromolecules in the PNIPAM brush stretch and absorb the solvent. We show that under these conditions, the adherence between the PNIPAM brush and a silicon oxide, gold, polystyrene and poly(methyl methacrylate) colloid attached to an atomic force microscopy cantilever is low. In contrast, when the PNIPAM brushes are in a collapse phase in a 30-70 vol.\% ethanol-water mixture, the adhesion between the brush and the different counter surfaces is high. We demonstrate that this difference in adhesion can be utilized to pick up 900 silicon oxide nanoparticles of diameter $80 \mathrm{~nm}$ using a 10x10 $\mathrm{mm}^{2}$ PNIPAM brush in 30-70 vol.\% ethanol-water mixture and release $98 \%$ of them in pure ethanol, while the percentage of aggregates, which all contain less than 20 particles, increases from only 2 to only $4 \%$. 


\subsection{Introduction}

Nanoparticles are nowadays broadly applied in personal hygiene products, ${ }^{1}$ catalysis, ${ }^{2}$ bioanalysis, ${ }^{3}$ drug-delivery ${ }^{4}$ and water-treatment. ${ }^{5}$ To prepare these nanoparticles, the solgel method, ${ }^{6}$ where the particles are formed in solution via hydrolysis and polycondensation reaction of the precursor, is most commonly employed. ${ }^{7}$ After synthesis, the nanoparticles need to be transferred to another medium for storage or further processing. Generally, this is realized via a two-step method. ${ }^{6 c, 7 b}$ First, the particles are precipitated from solution by, for example, ultra-centrifuge. Next, the particles from the aggregate are allowed to diffuse into the new medium for further modification. The dissolution of the nanoparticles from the aggregate is often difficult to realize, ${ }^{8}$ because of the relatively strong interfacial forces acting between these particles that have high surface-to-volume ratios. To enable the dispersion, many techniques are employed, such as chemical or physical surface modifications, ${ }^{9}$ and sonication. ${ }^{10}$ A smart alternative to these techniques is, however, the pick up and release of particles by employment of stimulus responsive polymer brushes, ${ }^{11}$ because within this method aggregation of the particles during the extraction is less likely to occur.

Polymer brushes consist of macromolecules that are attached with one end to a surface at a density that is high enough for the polymers to stretch away from the grafting surface. ${ }^{12}$ The degree of stretching of the polymers depends on the solvent quality. In poor solvents, the polymers form a dense layer at the surface, while the polymers stretch more strongly in good solvents. Adhesion between the brush and the counter-surface (CS) is usually low when brushes are swollen in a good solvent, ${ }^{13}$ especially when brush-solvent interactions are favored over brush-CS interactions. In poor solvents, the adhesion is generally much higher, because of the possibility of more direct interaction between the brush and the CS. ${ }^{13 a}$, 14 When the brushes are composed of polymers for which the effective solvent quality can be altered via an applied stimulus, such as temperature, $,{ }^{15} \mathrm{pH},{ }^{16}$ solvent, ${ }^{17}$ light,${ }^{18}$ salts, ${ }^{19}$ electronic ${ }^{20}$ and magnetic field, ${ }^{21}$ adhesion can be reversibly switched between high and low. ${ }^{13 c, 22}$

Poly( $N$-isopropyl acrylamide) PNIPAM is a stimulus responsive polymer. ${ }^{23}$ PNIPAM changes its conformation from an extended coil to a collapsed globule upon increasing the temperature above the lower critical solution temperature (LCST) of $32{ }^{\circ} \mathrm{C}$ in pure water. ${ }^{24}$ This effect can be employed the switch the adherence between PNIPAM brushes ${ }^{22 \mathrm{~d}}$ and PNIPAM and a different CSs. ${ }^{13 c,}{ }^{22 a}$ Reversible adherence changes by heating and cooling PNPAM have been applied to capture and release polymer particles, ${ }^{25}$ cells ${ }^{22 \mathrm{f}}$ and dye. ${ }^{26}$ However, for consideration of energy cost, it might not be feasible to change the temperature repeatedly and it could be more practical to alter the adherence by adjusting the solvent composition. 
Besides being temperature-responsive, PNIPAM can also be responsive to changes in the solvent composition. ${ }^{27}$ The LCST of PNIPAM shifts to lower temperatures in certain mixtures of two good solvents. For example, for methanol-water mixtures between 30 and 65 vol. $\%,{ }^{28}$ the LCST is lower than $20{ }^{\circ} \mathrm{C}$ and PNIPAM precipitates. This effect is called co-non-solvency and has been observed for PNIPAM in methanol-water, ${ }^{27 d,} 29$ tetrahydrofuran (THF)-water ${ }^{27 c, 30}$ and dimethyl formamide (DMF)-water ${ }^{31}$ mixtures too. Though the mechanism of co-non-solvency of PNIPAM is still under debate, ${ }^{27 a, 27 d, 32}$ the effect is already regularly employed in fermentation gating, ${ }^{33}$ regulating channel permeability, ${ }^{34}$ and force actuation. ${ }^{35}$

In this article, we show that co-non-solvency of PNIPAM can be utilized to capture nanoparticles from their aqueous solution and re-disperse them into the new solution. By systematically varying the solvent composition, we find that the change in adhesion between PNIPAM and different CSs is maximum upon changing the composition between pure ethanol (low) and 30-70 vol\% ethanol-water. The magnitude of the adherence-change depends on the CS, but is in all cases larger than $120 \%$. Using this method, we can pick up 9 silicon nanoparticles per $\mu \mathrm{m}^{2}$ of PNIPAM brush and redistribute them into a new solution. Moreover, we show that the percentage of aggregates is less than $2 \%$ and increases by less than $4 \%$ after release and the next pick up cycle. All aggregates we observed in the brushes are less than 20 particles. This shows that the presented method holds great potential for application in nanoparticle transfer between solutions without the formation of large aggregates.

\subsection{Materials and Methods}

\subsubsection{Materials}

$N$-Isopropylacrylamide (NIPAM, Aldrich, 97\%) was recrystallized twice in toluene/hexane mix solution (50\% v/v). Copper (I) bromide (CuBr, Aldrich, 98\%) was washed by acetic acid three times, and dried overnight. (3-aminopropyl)triethoxysilane (APTES), 2-bromo-2methylpropionyl bromide (BiBB, 98\%), triethylamine (TEA, $\geq 99 \%$ ), copper (II) bromide ( $\geq 99 \%), N, N, N^{\prime}, N^{\prime}, N^{\prime}$-pentamethyldiethylenetriamine (PMDETA) (98\%), were purchased from Sigma-Aldrich and used as received without any purification. The solvents used in the experiments, such as methanol (absolute), tetrahydrofuran (THF), toluene, hexane and sulfuric acid (95-97\%), were bought from Biosolve. Hydrogen peroxide and ethanol were purchased from Merck, and were used as received. Silicon nanoparticles $(D=80 \pm 15 \mathrm{~nm})$ were bought from Aldrich with $40 \mathrm{wt} . \%$ suspension in water. MilliQ water was made from a MilliQ Advantage A 10 purification system (Millipore, Billerica, Ma, USA). The

preparation of the PNIPAM brushes is the same as reported in the reference ${ }^{36}$ and according to a well-established recipe. ${ }^{37}$ First, vapor deposition was utilized to attach the APTES molecules to a piranha washed silicon surface. Next, a one-step reaction between amine 
group and BiBB was performed to link the initiator to the silane. The PNIPAM brushes were grafted from the surface using surface initiated atom transfer radical polymerization (SI-ATRP). ${ }^{38}$ For a movie showing the sample preparation procedure, see Ref JoVE. ${ }^{39}$

\subsubsection{Colloidal probe preparation}

The preparation of our colloidal probes is based on a regularly employed technique. ${ }^{40}$ Polystyrene (PS) spheres (Duke Standards 4205A, Thermo Scientific, diameter $5 \mu \mathrm{m}$ ) and $\mathrm{SiO}_{2}$ spheres (Cospheric, $\mathrm{SiO}_{2} \mathrm{MS}-1.8$, diameter $7.75 \mu \mathrm{m}$ ) were diluted in water and dropcoated on a freshly cleaved mica surface, and dried with nitrogen. Individual colloids were lifted up from the substrate by a tungsten wire (World Precision Instruments, Inc.) controlled by a home-made micromanipulator under an optical microscope. The tungsten wire was chemically etched in $1 \mathrm{M} \mathrm{KOH}$ water solution ( $30 \mathrm{~V}$, platinum electrode) until the end-diameter was of about 10-15 $\mu \mathrm{m}$ in order to transfer individual colloids only. Next, the tungsten wire was cleaned in ethanol and dried under nitrogen (wire-colloid capillary adhesion). The spheres were then fastened using a UV-cured glue (Optical Adhesive 81, Norland Products, Inc.) to an AFM cantilever (TL-CONT-50, Nanosensors) and photocured using high intensity UV lamp (Hamamatsu LC8, type 02A) for 3 minutes under air. The quality of the prepared colloidal probes was monitored by high-resolution optical imaging (Appendix 5.1). The gold colloid probes (6 $\mu \mathrm{m}$ diameter, CP-CONT-Au-C, sQube, NanoAndMore, Germany) were used directly and some of them were used to graft PMMA from the surface using SI-ATRP ${ }^{39}$ yielding a PMMA dry brush thickness of $236 \mathrm{~nm}$.

\subsubsection{Brush characterization}

The PNIPAM brushes were characterized by Fourier transform infrared spectroscopy (FTIR, Bruker Vertex 70v spectrometer), and the characteristic groups of PNIPAM are amide I and II appeared at 1632 and $1530 \mathrm{~cm}^{-1}$ respectively, which gave the evidence of PNIPAM brushes grafting on the substrates in Appendix 5.2. The brushes were degrafted from the surface in $p$-toluene sulfonic acid aqueous solution at $80^{\circ} \mathrm{C}^{41}$. Then the molecular weight was determined to be $M_{n}=1 \times 10^{5} \mathrm{~g} / \mathrm{mol}$ by using gel permeation chromatography (GPC) in THF. The grafting density is around 0.25 chains $/ \mathrm{nm}^{2}$ estimated from swelling ratio. ${ }^{2 \mathrm{~d}}$ AFM measurements were carried out on a Multimode 8 AFM with a NanoScope V controller, a JV vertical engage scanner and a liquid cell (Bruker, Santa Barbara, CA). Colloid attached to soft silicon cantilevers were employed to measure the swelling ratio and adhesion force at various ethanol-water compositions (typically $k=0.3 \mathrm{~N} / \mathrm{m} \pm 0.03$ ). We gently scratched off the brushes by using a Teflon tweezer, and the dry height was determined by measuring the relative height between bare silicon and brush surface. In various composition of ethanol-water mixture, a low force $(1.5 \mathrm{nN})$ was applied to contact with the brush to measure the swelling height, which was normalized by the dry thickness to give the swelling ratio. All the measurements were done on at least 3 different positions of the samples. The morphology of PNIPAM surface with attached silicon nanoparticles 
was scanned by tapping mode of AFM. Silica cantilevers (NanoWorld) with a resonance frequency of $320 \mathrm{kHz}$ and a spring constant of $42 \mathrm{~N} / \mathrm{m}$ were used.

\subsubsection{AFM adhesion force measurements}

To obtain the adhesion forces, we first approached the AFM cantilever towards the PNIPAM until a maximum repulsive force of $60 \mathrm{nN}$ was reached. The force was measured upon retract of the cantilever from the PNIPAM brush at a velocity of $0.8 \mu \mathrm{m} / \mathrm{s}$, where the adhesion force was defined as the minimum force before the colloid and the brush loose contact. The typical ramp curves in different solvent compositions are shown in Appendix 5.3. Each point is obtained by averaging over 30 force-distance curves captured on three different position on the same sample, where we found the same forces within $10 \%$. Experiments were also repeated on 3 different samples that were prepared in the same batch. For all these samples we found qualitatively the same results $(30 \%$ deviations in different samples).

\subsubsection{Particle transfer measurements}

Here, we dispersed silicon particles $(D=160 \mathrm{~nm})$ in ethanol-water $(\mathrm{v} / \mathrm{v}=3 / 7)$ solution, then a $1 \times 1 \mathrm{~cm}^{2}$ PNIPAM brush (dry height $=150 \mathrm{~nm}$ ) wafer was soaked in the mixture for 20 seconds. Next, the wafer was soaked in ethanol-water $(v / v=3 / 7)$ solution for 1 minute to remove the unattached particles. After gently blowing the sample to dry with nitrogen gun, the surface morphology was imaged by AFM with tapping mode. The release of particles are conducted by sonicating the sample in water or ethanol for 2 minutes in an ultrasonic cleaner (Branson 2510DTH). Then the wafer was dried and used for AFM measurement. For comparison experiments, the PNIPAM brush was used to pick up and release silicon particles from aqueous solution. Meanwhile, bare silicon wafer also was used for comparison. In order to get a high resolution of image, the scan size is confined in $3.3 \mu \mathrm{m}$ with a scan frequency of $0.2 \mathrm{~Hz}$. The number of particles were counted by using ImageJ.

\subsection{Results and Discussion}

Figure 5.1 shows the adhesive force and brush swelling for a PNIPAM brush (dry height 75 $\mathrm{nm}$ ) measured by a silicon colloid probe in different ethanol-water mixtures between 0-100 vol.\% ethanol. The variation in swelling ratio with the ethanol content in the solvent mixture shows qualitatively the same trend as in our recent publication, where we compared different measurement technique to extract brush swelling. ${ }^{13 b}$ The swelling ratio of the PNIPAM brush in pure water (3.4) is slightly higher than in Ref. ${ }^{13 b}$ (3.0), which is probably due to the lower grafting density ${ }^{22 \mathrm{~d}}$ of the measured samples $\left(0.25\right.$ chains $/ \mathrm{nm}^{2}$ here vs 0.45 chains $/ \mathrm{nm}^{2}$ in Ref. $\left.{ }^{13 \mathrm{~b}}\right)$. For the same reason, the swelling ratio of our PNIPAM brush in ethanol (4.3) is also slightly higher than in Ref. ${ }^{13 b}$ (3.6). 


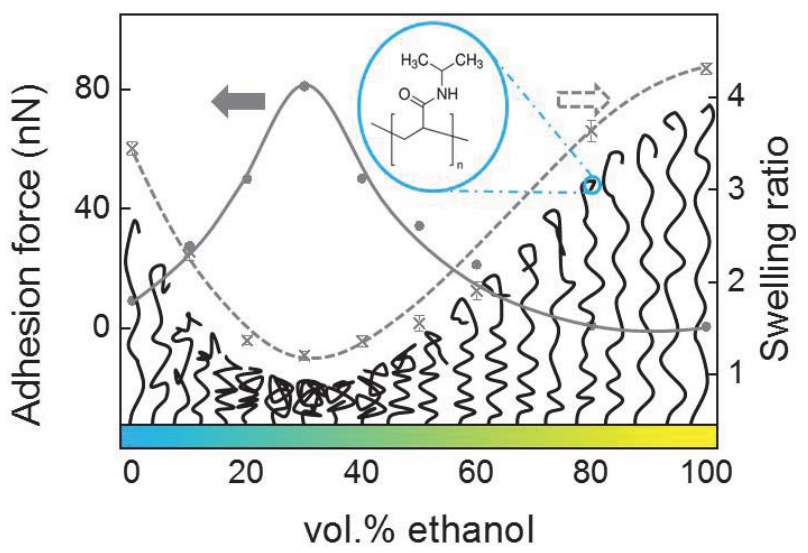

Figure 5.1. The PNIPAM swelling ratio and adhesion force between PNIPAM and a $\mathrm{SiO}_{2}$ colloid (diameter $7.75 \mu \mathrm{m}$ ) in various compositions of ethanol-water mixtures. The swelling ratio is calculated from the swollen height of brushes (measured by imaging under a load of $1.5 \mathrm{nN}$ ), which is normalized by the dry height of the brush. The adhesion force is obtained from the force separation curves measured for a retraction velocity of $0.8 \mu \mathrm{m} / \mathrm{s}$ after the colloid has been pushed into the brush with a normal force of $60 \pm 6 \mathrm{nN}$.

We find that the brush swelling is minimum for $30 \mathrm{vol} \%$ ethanol in the water-ethanol mixture. In this minimum the swelling ratio is still 1.2, which indicates that there is a significant amount of solvent trapped in the brush. The entrapment of solvent in the brush is consistent with the picture that polymer collapse is induced by bridging ${ }^{32 \mathrm{~d}}$ between the hydrophobic groups of the PNIPAM monomers at different parts of the polymer ${ }^{32 a}$ via molecules of the better solvent (ethanol). Also, recently published attenuated total reflection infrared spectroscopy (ATR IR) measurements on a poly(dodecyl methacrylate) brush in mixtures of toluene and ethanol have shown preferential absorption by the better solvent (toluene). ${ }^{42}$ This would indicate that there could be an ethanol-rich phase in our PNIPAM brush. But, unfortunately, we cannot extract the chemical composition of the solvent in our brush and, thus, this remains to be speculation.

Figure 5.1 also shows that the adhesive force between the PNIPAM brush and a $\mathrm{SiO}_{2}$ colloid also strongly varies with the solvent composition. In pure water, the adhesion is low $(8.1 \mathrm{nN})$, because the solvent screens the interactions between the colloid and the polymers in the brush. Upon increasing the ethanol content to 10 vol. \%, the brush swelling strongly decreases by $32 \%$ and the adhesion force increases to $27 \mathrm{nN}$. The increase in adhesion can be explained by the increase in polymer density in the contact as a consequence of the reduced brush swelling. For the 10 vol.\% ethanol mixture, we also observe a long-ranged attractive interaction over $1.6 \mu \mathrm{m}$ (Appendix 5.3), which we attribute to polymer stretching. ${ }^{13 \mathrm{~b}, 13 \mathrm{c}, 43}$ Polymer stretching has been shown to result in high friction. ${ }^{13 \mathrm{c}} \mathrm{We}$ find that the adhesion is the highest $(81 \mathrm{nN})$ at $30 \mathrm{vol} \%$ ethanol in the ethanol-water mixture. Upon further increase of the ethanol content in the solvent mixture, the adhesion decreases and it is minimum in pure ethanol $(0.7 \mathrm{nN})$ where the brush swells the most. 
Table 5.1 The average adhesion force $F_{\text {adh }}$ measured in pure water $(0 \%), 30-70$ vol. \% ethanolwater mixture $(30 \%)$ and in pure ethanol $(100 \%)$ using colloid probes of different sizes and materials, while keeping the retraction velocity $(0.8-1 \mu \mathrm{m} / \mathrm{s})$ and the maximum indentation force $F_{\text {max }}$ divided by the colloid diameter $D$ approximately constant.

$\begin{array}{cccccc}\text { Material } & \boldsymbol{D}(\boldsymbol{\mu} \mathbf{m}) & \boldsymbol{F}_{\max }(\mathbf{n N}) & \boldsymbol{F}_{\text {adh }} \mathbf{0} \%(\mathbf{n N}) & \boldsymbol{F}_{\text {adh }} \mathbf{3 0} \%(\mathbf{n N}) & \boldsymbol{F}_{\text {adh }} \mathbf{1 0 0} \%(\mathbf{n N}) \\ \mathrm{SiO}_{2} & 7.75 & 77.5 & 8.1 & 81 & 0.7 \\ \mathrm{Au} & 6 & 60 & 1.6 & 73 & 0.2 \\ \mathrm{PS} & 5 & 50 & 0.5 & 33.4 & 0.2 \\ \text { PMMA } & 6 & 60 & 0.05 & 12 & 0.08\end{array}$

Figure 5.2 shows the adhesion force between the $\mathrm{SiO}_{2}$ colloid and PNIPAM brush by consecutively varying 4 times the composition of the solvent mixture between $100 \%$ ethanol and 30-70 vol.\% ethanol-water. Between each solvent exchange the brush surface is washed with ethanol and dried under a stream of nitrogen. The results in Figure 5.2 show that the adhesive force can be switched from $0.7 \pm 0.1 \mathrm{nN}$ in pure ethanol to $81 \pm 10 \mathrm{nN}$ for $30 \mathrm{vol} \%$ of ethanol in the solvent mixture. After 4 cycles, the change in adhesion is still the same within the experimental error without showing any signs of degradation. The repeatability of the adhesion-switch shows that our system holds great potential for application in pick up and release systems.

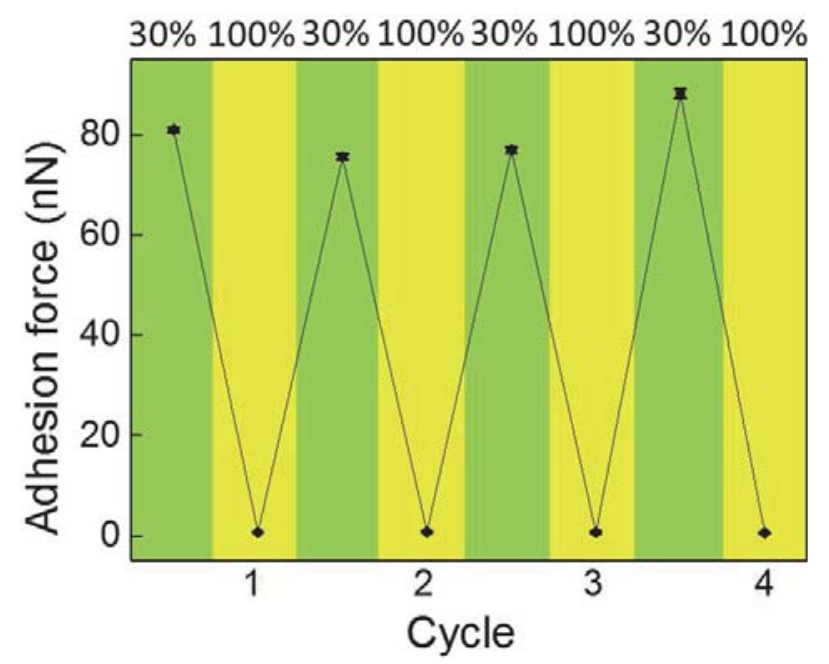

Figure 5.2. Repeatability test showing that the adhesion force can be switched from $81 \pm$ $10 \mathrm{nN}$ in $30-70 \mathrm{vol} \%$ ethanol-water mixture to $0.7 \pm 0.1 \mathrm{nN}$ in ethanol and back for 4 cycles without showing signs of degradation. The error bars denote the standard error of the mean with a $95 \%$ confidence interval.

Appendix 5.3 shows the typical ramp curves between a PNIPAM brush and a gold colloid. We also use other colloids for the force-separation measurement, such as polystyrene (PS), 
and poly(methyl methacrylate) (PMMA) coated gold colloid. The results for all studied counter surfaces are summarized in Table 5.1. Though the absolute values for adhesion forces are different for the different colloid materials, we observe the same trend in the adhesion force as a function of the solvent composition for all counter surfaces. For all colloid materials, the adhesion is highest for an ethanol-water mixture that contains 30 vol.\% ethanol, while the adhesion is much lower in the pure solvents. We find the largest change in adhesion for the gold colloid. For gold, the adhesion in pure ethanol is a factor 365 lower than in 30-70 vol.\% ethanol-water. The overall adhesion between the PMMA colloid and the PNIPAM brush is much lower than the adhesion for the other colloid materials. We attribute this reduction to a small preference of the solvent to move into the PMMA film, ${ }^{22 g}$ which could screen direct colloid-brush interactions. In bulk PMMA, there is a 2-4 vol.\% solvent absorbance of pure water, ${ }^{44}$ which increases to $20 \mathrm{vol} \%$ in $30-70$ vol.\% ethanol-water. ${ }^{45}$ Due to a cosolvency effect of PMMA in ethanol-water mixtures, ${ }^{22 g}$ there is a solubility-maximum at $80-20$ vol.\% ethanol-water. For this composition, there is approximately 60 vol.\% of solvent absorbed in the PMMA film. This might be the reason for the slightly lower adherence around 80-20 vol.\% ethanol-water. Nevertheless, for all counter surfaces the adhesion can be switched by at least a factor 115 , which shows that our system will be able to pick up and release different kinds of nanoparticles.

Figure 5.3 shows the pick up and release of silicon particles (diameter $80 \mathrm{~nm}$ ) using a 1x1 $\mathrm{cm}^{2}$ PNIPAM brush (dry height $=150 \mathrm{~nm}$ ). The particles are picked up in a 30-70 vol.\% ethanol-water mixture. The release of particles is allowed by ultra-sonication in ethanol for 2 minutes. Figure 5.3. (a) is an AFM height image of the brush before immersion in solution showing that the brush is clean and homogeneous (RMS roughness $0.3 \mathrm{~nm}$ ). In (b) we show the AFM height image of the PNIPAM brush after it has been immersed in a nanoparticle solution (concentration $1 \mathrm{mg} / \mathrm{ml}$ ) in 30-70 vol.\% ethanol-water and then dried. In this pick up image, there are 90 nanoparticles that are spread out over an area of $3.3 \times 3.3$ $\mu \mathrm{m}^{2}$. The particles are evenly distributed over the surface, which shows that there is little aggregation of the nanoparticles. Only 20 clusters of less than $2 \%$ particles are observable within this part of the brush. In Figure 5.3. (c) the AFM image of the brush after particlerelease is shown. The brush is clean and homogeneous and there are no particles left on this part of the surface, which shows that the particles can be successfully transferred from one medium into another. In contrast, if the brush is sonicated in 30-70 vol.\% ethanol-water, only $5-8 \%$ of the particles are released. 


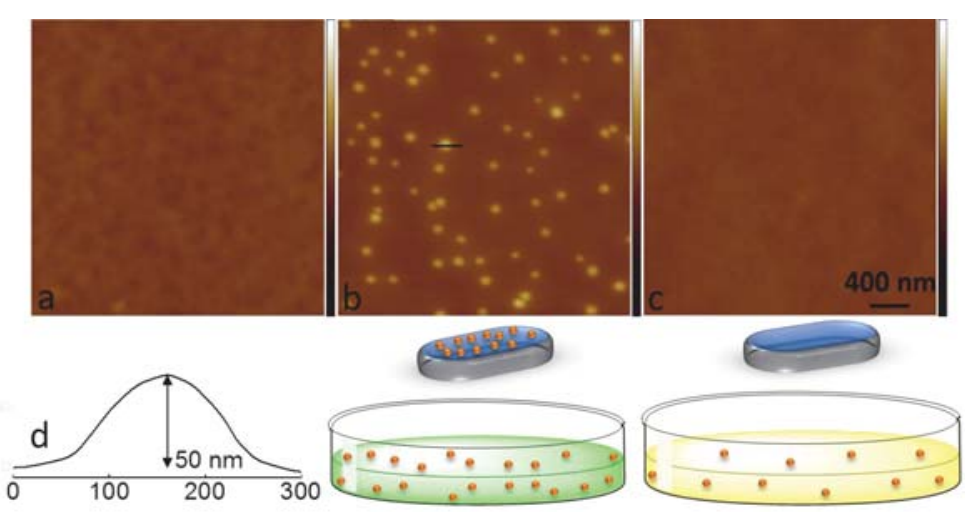

Figure 5.3. AFM imaging of the dry PNIPAM brush surface of (a) before adsorption of silicon particles, (b) after immersion in 30-70 vol.\% ethanol-water mixture containing silicon nanoparticles (diameter $80 \mathrm{~nm}, 1 \mathrm{mg} / \mathrm{ml}$ ) and (c) the same PNIPAM brush surface after immersion in pure ethanol and sonication for 2 minutes. The black line in (b) denotes the positon of the cross section shown in (d). The dry brush height is $150 \mathrm{~nm}$, and the scale bar is $400 \mathrm{~nm}$.

The graph in Figure 5.3. (d) shows a cross section of a nanoparticle that is partly embedded in the dried PNIPAM brush. Approximately $62 \%$ of the particle's volume is in the brush, the other part is exposed to the air. The penetration depth of nanoparticles into a brush depends on the size ${ }^{46}$ and geometry ${ }^{47}$ of the particles and the solvent-mediated particlepolymer interactions. ${ }^{48}$ Particles that are smaller than or comparable to the distance between anchor points of the polymers are free to move into the brush. These small particles can self-organize and form clusters inside the brush. ${ }^{48-49}$ Our particles are approximately 60 times larger than the distance between the anchor points and therefore our particles reside in the top of the brush and there is no significant aggregation or cluster formation.

To test the performance of our PNIPAM pick up and release system, we compare the amount of captured particles from our 30-70 vol.\% ethanol-water, to the number of captured particles on PNIPAM in pure water (see Appendix 5.4), which shows a higher adhesion than in pure ethanol (see Table 5.1) for PNIPAM in contact with $\mathrm{SiO}_{2}$ colloid and on a bare silicon surface (see Appendix 5.5). Figure 5.4. (a) shows the average number of particles imaged over $10 \times 10 \mu^{2}$ (see Appendix 5.6) for these three test situations. The number of trapped particles for the PNIPAM brush in 30-70 vol.\% ethanol-water is approximately 25 times higher than for PNIPAM in pure water and 90 times higher than for the bare silicon surface.

To test if the number of aggregates changes after release from the brush, we performed experiments of 4 consecutive capture and release cycles. The inset of Figure 5.4 shows that there are on average 20 aggregates visible per image, which is 2 percent of the total number of particles. Moreover, the results in the inset show that there is no change in the number of aggregates in the picture within the experimental error. However, the total number of 
picked up particles decreases per cycle (as shown in Appendix 5.7) such that the percentage of aggregate increases to $4 \%$ after 4 cycles. We excluded brush degradation as the reason for the reduction in entrapment efficiency (see Appendix 5.8) and, instead, attribute it to the reduction in concentration after several pick up and release cycles.
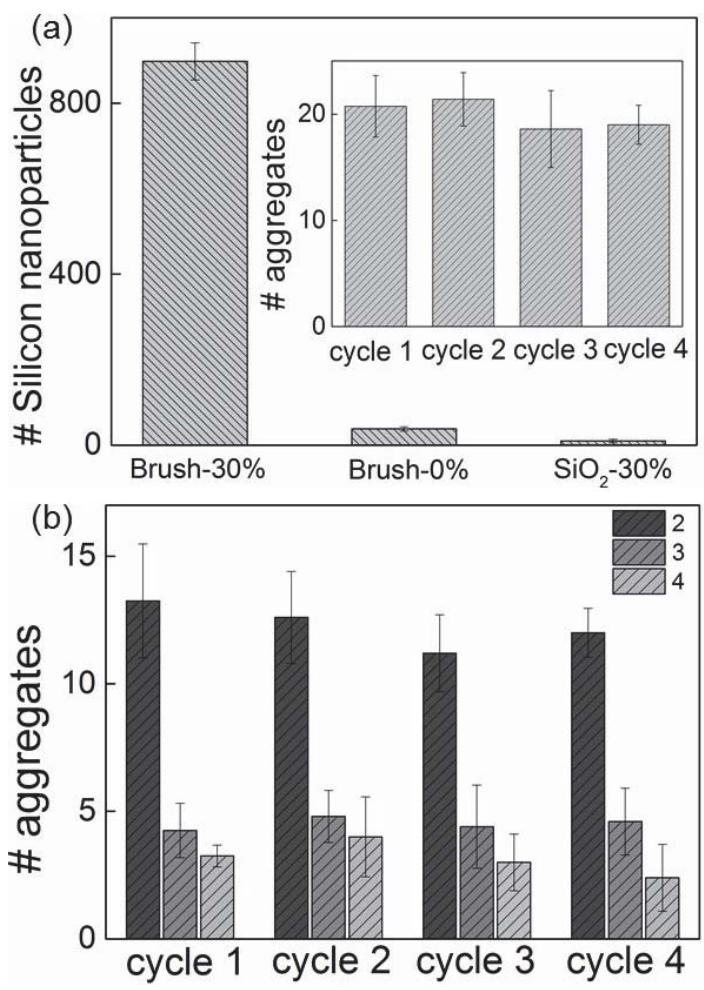

Figure 5.4. (a) The amount of particles attached on the brush or $\mathrm{SiO}_{2}$ substrates after immersion in ethanol-water solution $(\mathrm{v} / \mathrm{v}=3 / 7)$ containing silicon particles (concentration $1 \mathrm{mg} / \mathrm{ml}$ ), pure water containing silicon particles (concentration $1 \mathrm{mg} / \mathrm{ml}$ ). The image size is $10 \times 10 \mu \mathrm{m}^{2}$. The inset shows the number of aggregates in the image during 4 consecutive capture and release cycles using ethanol-water solution $(\mathrm{v} / \mathrm{v}=3 / 7)$ and pure ethanol. (b) The number of particles in the aggregates during the 4 consecutive capture and release cycles.

\subsection{Conclusions}

In summary, we have shown that the adhesion force between a PNIPAM brush and $\mathrm{SiO}_{2}$, gold, PS and PMMA colloids can be reversibly switched by changing the solvent composition. In 30-70 vol.\% ethanol-water mixture adhesion is high, while in pure solvents the adhesion is low. We employ the change in adherence to build a pick up move and release system to transfer particles from one liquid into another. The advantage of our system is that the formation of aggregates is strongly reduced, which is an important problem in traditional method of nanoparticle transfer. 


\subsection{Appendix}

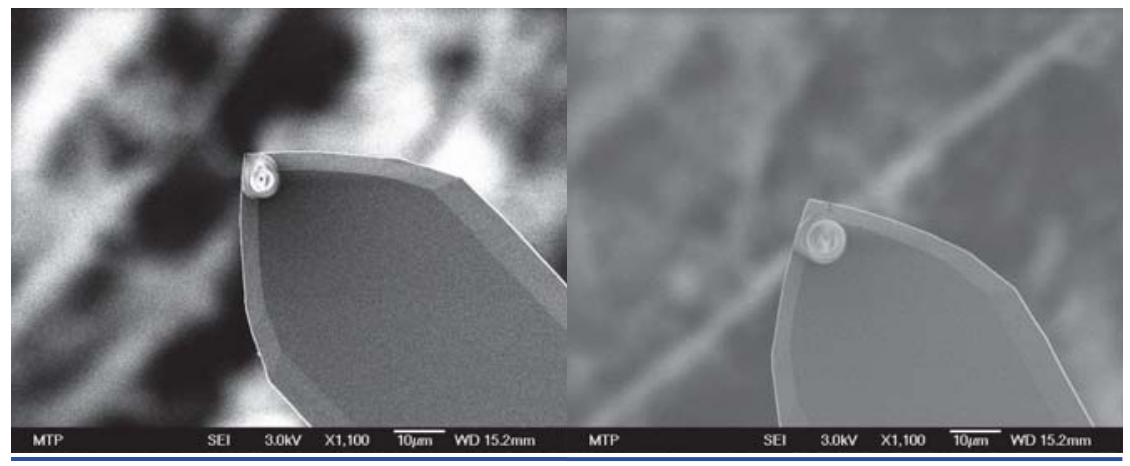

Appendix 5.1. SEM image of typical PS and silica colloid cantilevers, the diameter is around 5 and $7.75 \mu \mathrm{m}$ respectively.

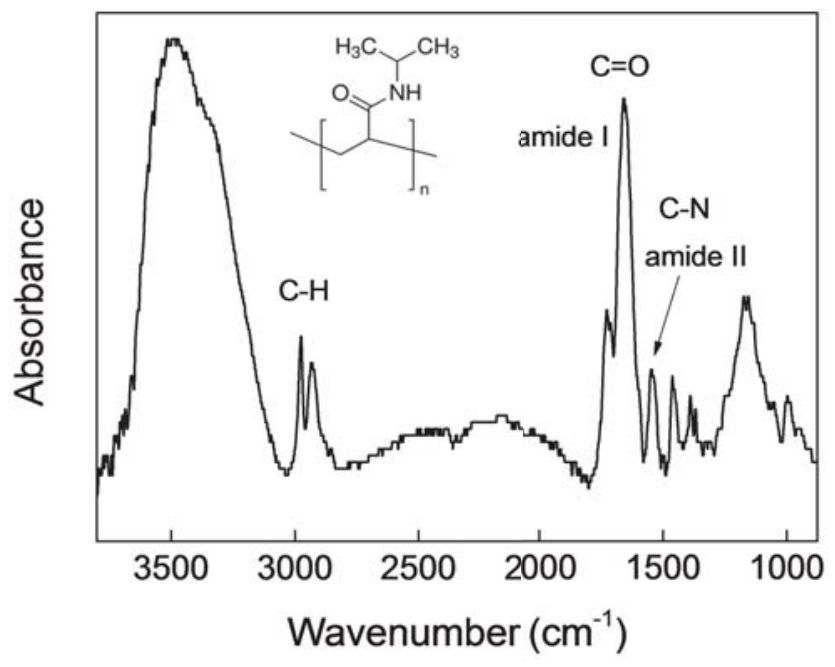

Appendix 5.2. FTIR spectrum of PNIPAM brushes 


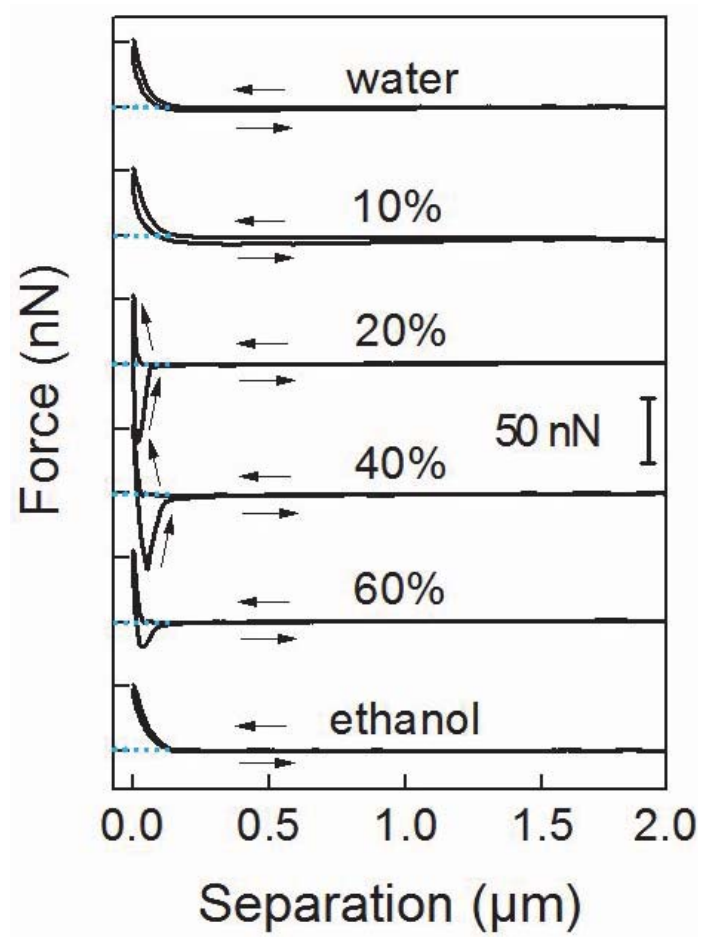

Appendix 5.3. Typical ramp curve at various ethanol volume fraction in water $(0,10,20$, $40,60,100 \%$ ). The approach and retract velocity is $0.8 \mu \mathrm{m} / \mathrm{s}$, and the ramp size is $2 \mu \mathrm{m}$. The scale bar equals $60 \mathrm{nN}$.

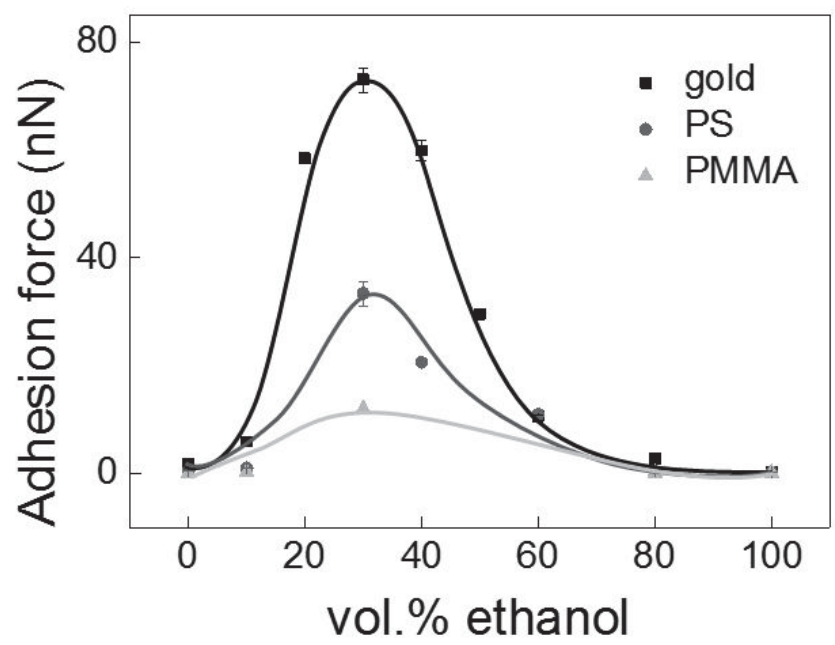

Appendix 5.4. The adhesion force between PNIPAM brush and a colloid (gold, PS, PMMA) in various compositions of ethanol-water mixtures. The adhesion force is obtained from the force separation curves measured for a retraction velocity of $0.8 \mu \mathrm{m} / \mathrm{s}$ after the colloid has been pushed into the brush with a normal force of $60 \mathrm{nN}$. The solid line is guide for the eye. 

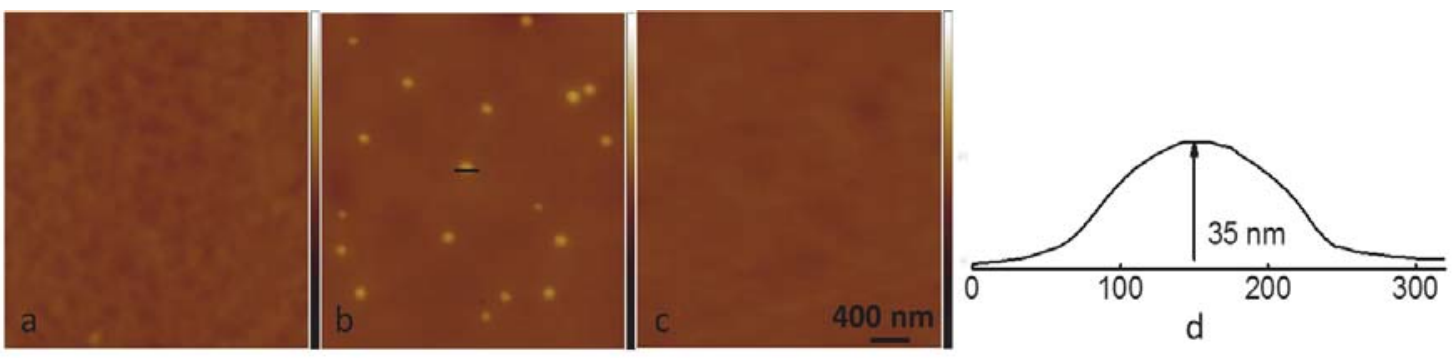

Appendix 5.5. AFM imaging of PNIPAM surface (a), soaking in silicon particles water solution (b), after sonication for 2 minutes (c), particle height (d). The dry PNIPAM brush is $150 \mathrm{~nm}$, and the scale bar is $400 \mathrm{~nm}$.
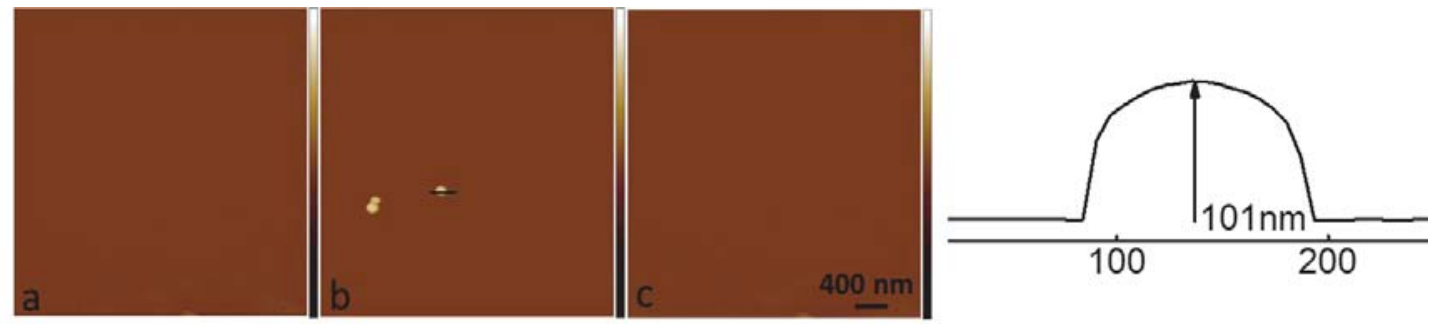

Appendix 5.6. AFM imaging of silicon surface (a), soaking in silicon particles aqueous solution with $30 \%$ ethanol volume fraction (b), after sonication for 2 minutes (c), particle height (d). The dry PNIPAM brush is $150 \mathrm{~nm}$, and the scale bar is $400 \mathrm{~nm}$. 


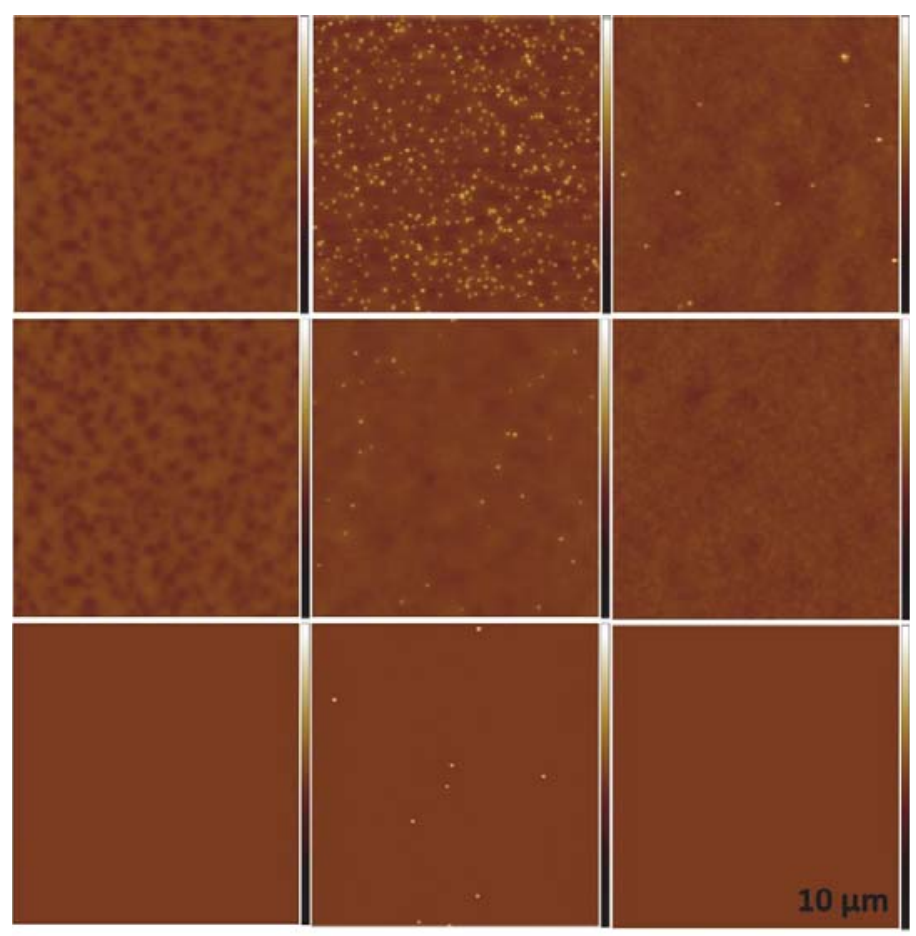

Appendix 5.7. Statistic of silicon particles by PNIPAM brush, PNIPAM surface (a), soaked in silicon aqueous solution for 20 seconds (b), after sonication in ethanol for 2 minutes (c), PNIPAM surface before soaking (d), soaked in silicon aqueous solution with $30 \%$ ethanol for 20 seconds (e), after sonication in ethanol for 2 minutes (f), silicon surface (g), soaked in silicon aqueous solution with $30 \%$ ethanol for 20 seconds (h), after sonication in ethanol for 2 minutes (i). The dry PNIPAM brush is $150 \mathrm{~nm}$, and the scale bar is $1 \mu \mathrm{m}$.

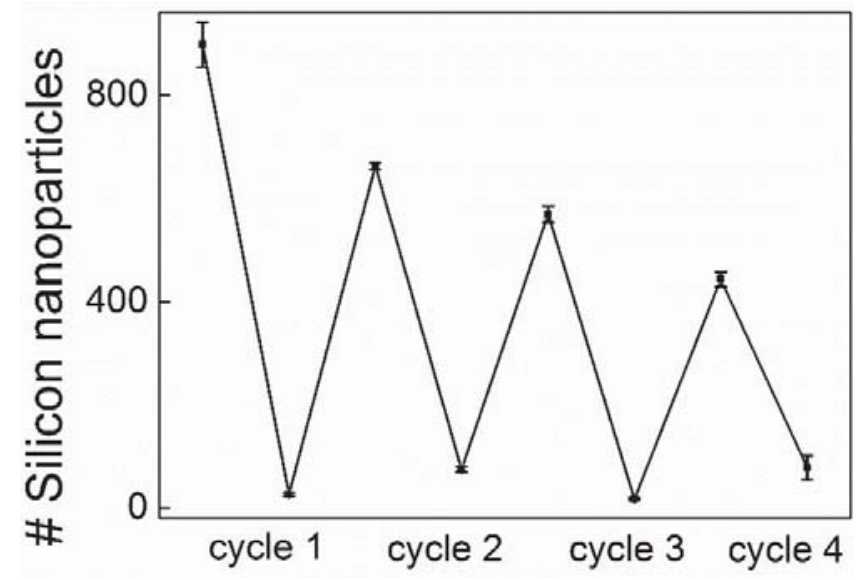

Appendix 5.8. Cycles of numbers of picking up silicon particles using PNIPAM brush at $30 \%$ volume fraction of ethanol and release with sonication in ethanol for 2 minutes 


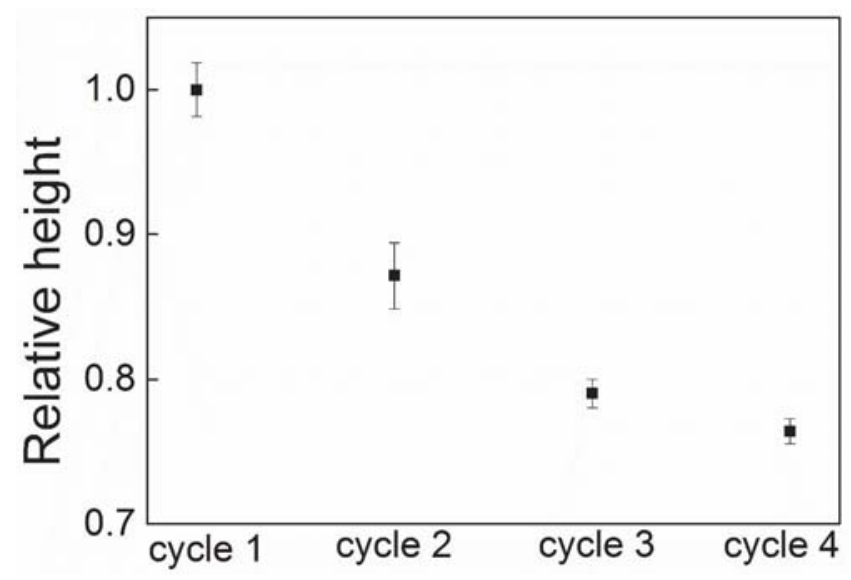

Appendix 5.9. The relative dry height change of PNIPAM brush after sonicating in ethanol for 2 minutes for 4 cycles. 


\subsection{References}

[1] a) J. F. Wang, T. Tsuzuki, L. Sun and X. G. Wang, Acs Applied Materials \& Interfaces 2010, 2, 957-960; b) D. M. Eby, H. R. Luckarift and G. R. Johnson, Acs Applied Materials \& Interfaces 2009, 1, 1553-1560; c) I. Perelshtein, G. Applerot, N. Perkas, E. Wehrschetz-Sigl, A. Hasmann, G. M. Guebitz and A. Gedanken, Acs Applied Materials \& Interfaces 2009, 1, 363-366.

[2] a) L. Shang, T. Bian, B. H. Zhang, D. H. Zhang, L. Z. Wu, C. H. Tung, Y. D. Yin and T. R. Zhang, Angewandte Chemie-International Edition 2014, 53, 250-254; b) F. Costantini, E. M. Benetti, D. N. Reinhoudt, J. Huskens, G. J. Vancso and W. Verboom, Lab on a Chip 2010, 10, 3407-3412.

[3] a) R. P. Bagwe, C. Y. Yang, L. R. Hilliard and W. H. Tan, Langmuir 2004, 20, 8336-8342; b) R. P. Bagwe, L. R. Hilliard and W. H. Tan, Langmuir 2006, 22, 4357-4362.

[4] R. A. French, A. R. Jacobson, B. Kim, S. L. Isley, R. L. Penn and P. C. Baveye, Environmental Science \& Technology 2009, 43, 1354-1359.

[5] a) M. Pena, X. G. Meng, G. P. Korfiatis and C. Y. Jing, Environmental Science \& Technology 2006, 40, 1257-1262; b) N. L. Torad, M. Hu, S. Ishihara, H. Sukegawa, A. A. Belik, M. Imura, K. Ariga, Y. Sakka and Y. Yamauchi, Small 2014, 10, 2096-2107.

[6] a) C. Liu, B. S. Zou, A. J. Rondinone and Z. J. Zhang, Journal of the American Chemical Society 2001, 123, 4344-4345; b) Y. Lu, Y. Yin, B. T. Mayers and Y. Xia, Nano letters 2002, 2 , 183-186; c) B. G. Trewyn, I. I. Slowing, S. Giri, H. T. Chen and V. S. Y. Lin, Accounts of Chemical Research 2007, 40, 846-853.

[7] a) Y. G. Lee, J. H. Park, C. Oh, S. G. Oh and Y. C. Kim, Langmuir 2007, 23, 10875-10878; b) B. L. Cushing, V. L. Kolesnichenko and C. J. O'Connor, Chemical Reviews 2004, 104, 3893-3946.

[8] M. Grzelczak, J. Vermant, E. M. Furst and L. M. Liz-Marzán, ACS nano 2010, 4, 3591-3605.

[9] a) C. Vauthier, B. Cabane and D. Labarre, European Journal of Pharmaceutics and

Biopharmaceutics 2008, 69, 466-475; b) A. Frattini, N. Pellegri, D. Nicastro and O. de Sanctis, Materials Chemistry and Physics 2005, 94, 148-152.

[10] R. Konwarh, N. Karak, C. E. Sawian, S. Baruah and M. Mandal, Carbohydrate Polymers 2011, $83,1245-1252$.

[11] S. Diamanti, S. Arifuzzaman, J. Genzer and R. A. Vaia, Acs Nano 2009, 3, 807-818.

[12] S. T. Milner, Science 1991, 251, 905-914.

[13] a) Q. Chen, E. S. Kooij, X. F. Sui, C. J. Padberg, M. A. Hempenius, P. M. Schon and G. J. Vancso, Soft Matter 2014, 10, 3134-3142; b) Y. Yu, M. Cirelli, B. D. Kieviet, E. S. Kooij, G. J.

Vancso and S. de Beer, Polymer 2016, 102, 372-378; c) Y. Yu, B. D. Kieviet, F. Liu, I. Siretanu, E. Kutnyanszky, G. J. Vancso and S. de Beer, Soft Matter 2015, 11, 8508-8516.

[14] a) Z. Y. Zhang, A. J. Morse, S. P. Armes, A. L. Lewis, M. Geoghegan and G. J. Leggett, Langmuir 2011, 27, 2514-2521; b) Z. Y. Zhang, A. J. Morse, S. P. Armes, A. L. Lewis, M.

Geoghegan and G. J. Leggett, Langmuir 2013, 29, 10684-10692; c) Q. B. Wei, M. R. Cai, F. Zhou and W. M. Liu, Macromolecules 2013, 46, 9368-9379.

[15] H. J. Dai, Q. Chen, H. L. Qin, Y. Guan, D. Y. Shen, Y. Q. Hua, Y. L. Tang and J. Xu, Macromolecules 2006, 39, 6584-6589.

[16] T. Wu, P. Gong, I. Szleifer, P. Vlc`ek, V. Šubr and J. Genzer, Macromolecules 2007, 40, 87568764.

[17] M. K. Vyas, K. Schneider, B. Nandan and M. Stamm, Soft Matter 2008, 4, 1024-1032.

[18] H. S. Lim, D. Kwak, D. Y. Lee, S. G. Lee and K. Cho, Journal of the American Chemical Society 2007, 129, 4128-4129.

[19] Y. Zhang, S. Furyk, L. B. Sagle, Y. Cho, D. E. Bergbreiter and P. S. Cremer, The Journal of Physical Chemistry C 2007, 111, 8916-8924.

[20] R. Jakubiak, T. J. Bunning, R. A. Vaia, L. V. Natarajan and V. P. Tondiglia, Advanced Materials 2003, 15, 241-244.

[21] J. M. Shen, F. Y. Gao, L. P. Guan, W. Su, Y. J. Yang, Q. R. Li and Z. C. Jin, Rsc Advances 2014, 4, 18473-18484.

[22] a) D. M. Jones, J. R. Smith, W. T. S. Huck and C. Alexander, Advanced Materials 2002, 14, 1130-1134; b) J.-K. Chen, J.-H. Wang, J.-Y. Chang and S.-K. Fan, Applied Physics Letters 2012, 
101, 123701; c) S. H. Ma, D. A. Wang, Y. M. Liang, B. Q. Sun, S. N. Gorb and F. Zhou, Small 2015, 11, 1131-1137; d) I. B. Malham and L. Bureau, Langmuir 2010, 26, 4762-4768; e) C. Rodriguez-Emmenegger, C. M. Preuss, B. Yameen, O. Pop-Georgievski, M. Bachmann, J. O. Mueller, M. Bruns, A. S. Goldmann, M. Bastmeyer and C. Barner-Kowollik, Advanced Materials 2013, 25, 6123-6127; f) X. F. Sui, A. Di Luca, M. K. Gunnewiek, E. S. Kooij, C. A. van Blitterswijk, L. Moroni, M. A. Hempenius and G. J. Vancso, Australian Journal of Chemistry 2011, 64, 1259-1266; g) Y. L. Yu, B. D. Kieviet, E. Kutnyanszky, G. J. Vancso and S. de Beer, Acs Macro Letters 2015, 4, 75-79; h) E. M. Benetti, S. Zapotoczny and J. Vancso, Advanced Materials 2007, 19, 268-271; i) S. de Beer, Langmuir 2014, 30, 8085-8090; j) S. de Beer, E. Kutnyanszky, P. M. Schon, G. J. Vancso and M. H. Muser, Nature Communications 2014, 5, 3781.

[23] a) P. W. Zhu and D. H. Napper, Journal of Colloid and Interface Science 1996, 177, 343-352;

b) T. Gilanyi, I. Varga, R. Meszaros, G. Filipcsei and M. Zrinyi, Physical Chemistry Chemical Physics 2000, 2, 1973-1977; c) J. Zhang, L. Y. Chu, Y. K. Li and Y. M. Lee, Polymer 2007, 48, 1718-1728.

[24] a) R. Liu, M. Fraylich and B. R. Saunders, Colloid and Polymer Science 2009, 287, 627-643; b) T. G. McKenzie, E. H. Wong, Q. Fu, S. J. Lam, D. E. Dunstan and G. G. Qiao, Macromolecules 2014, 47, 7869-7877.

[25] R. Liu, P. De Leonardis, N. Tirelli and B. R. Saunders, Journal of Colloid and Interface Science 2009, 340, 166-175.

[26] J. Guo, W. Yang, Y. Deng, C. Wang and S. Fu, small 2005, 1, 737-743.

[27] a) F. Tanaka, T. Koga and F. M. Winnik, Physical review letters 2008, 101, 028302; b) F. M. Winnik, M. F. Ottaviani, S. H. Bossmann, M. Garcia-Garibay and N. J. Turro, Macromolecules 1992, 25, 6007-6017; c) F. M. Winnik, M. F. Ottaviani, S. H. Bossmann, W. Pan, M. GarciaGaribay and N. J. Turro, Macromolecules 1993, 26, 4577-4585; d) D. Mukherji and K. Kremer, Macromolecules 2013, 46, 9158-9163.

[28] F. M. Winnik, H. Ringsdorf and J. Venzmer, Macromolecules 1990, 23, 2415-2416.

[29] C. Scherzinger, A. Schwarz, A. Bardow, K. Leonhard and W. Richtering, Current Opinion in Colloid \& Interface Science 2014, 19, 84-94.

[30] C. W. Tu and S. W. Kuo, Journal of Polymer Research 2014, 21.

[31] P. W. Zhu and D. H. Napper, Chemical physics letters 1996, 256, 51-56.

[32] a) F. Rodriguez-Ropero, T. Hajari and N. F. A. van der Vegt, Journal of Physical Chemistry B 2015, 119, 15780-15788; b) M. Z. Liu, F. L. Bian and F. L. Sheng, European Polymer Journal 2005, 41, 283-291; c) D. Mukherji, C. M. Marques and K. Kremer, Nature communications 2014, 5, 4882; d) D. Mukherji, C. M. Marques, T. Stuehn and K. Kremer, The Journal of chemical physics $\mathbf{2 0 1 5}$, $142,114903$.

[33] Y. Ito, T. Ito, H. Takaba and S. Nakao, Journal of Membrane Science 2005, 261, 145-151.

[34] Y. M. Sun, W. Wang, Y. Y. Wei, N. N. Deng, Z. Liu, X. J. Ju, R. Xie and L. Y. Chu, Lab on a Chip 2014, 14, 2418-2427.

[35] L. Liu, X. L. Song, X. J. Ju, R. Xie, Z. Liu and L. Y. Chu, Journal of Physical Chemistry B 2012, 116, 974-979.

[36] Y. L. Yu, B. D. Kieviet, F. Liu, I. Siretanu, E. Kutnyanszky, G. J. Vancso and S. de Beer, Soft Matter 2015, 11, 8508-8516.

[37] X. F. Sui, Q. Chen, M. A. Hempenius and G. J. Vancso, Small 2011, 7, 1440-1447.

[38] K. Matyjaszewski, Macromolecules 2012, 45, 4015-4039.

[39] S. de Beer, E. Kutnyanszky, M. H. Muser and G. J. Vancso, Jove-Journal of Visualized Experiments 2014, 52285.

[40] Y. Gan, Review of Scientific Instruments 2007, 78.

[41] C. Cummings, H. Murata, R. Koepsel and A. J. Russell, Biomaterials 2013, 34, 7437-7443.

[42] C. H. Mathis, M. Divandari, R. Simic, V. V. Naik, E. M. Benetti, L. Isa and N. D. Spencer, Langmuir 2016, 32, 7588-7595.

[43] J. D. Willott, T. J. Murdoch, G. B. Webber and E. J. Wanless, Macromolecules 2016, 49, 23272338.

[44] R. Hoogenboom, C. R. Becer, C. Guerrero-Sanchez, S. Hoeppener and U. S. Schubert, Australian journal of chemistry 2010, 63, 1173-1178. 
[45] S. M. Lee and Y. C. Bae, Polymer 2014, 55, 4684-4692.

[46] a) J. U. Kim and B. O'Shaughnessy, Macromolecules 2006, 39, 413-425; b) A. Milchev, D. I. Dirnitrova and K. Binder, Polymer 2008, 49, 3611-3618.

[47] S. de Beer, L. I. S. Mensink and B. D. Kieviet, Macromolecules 2016, 49, 1070-1078.

[48] O. A. Guskova, S. Pal and C. Seidel, Epl 2009, 88.

[49] a) R. Oren, Z. Q. Liang, J. S. Barnard, S. C. Warren, U. Wiesner and W. T. S. Huck, Journal of the American Chemical Society 2009, 131, 1670-1671; b) T. Curk, F. J. Martinez-Veracoechea, D. Frenkel and J. Dobnikar, Soft Matter 2013, 9, 5565-5571. 


\section{Chapter 6}

\section{Cosolvency-Induced Switching of the Adhesion between Poly(methyl methacrylate) Brushes*}
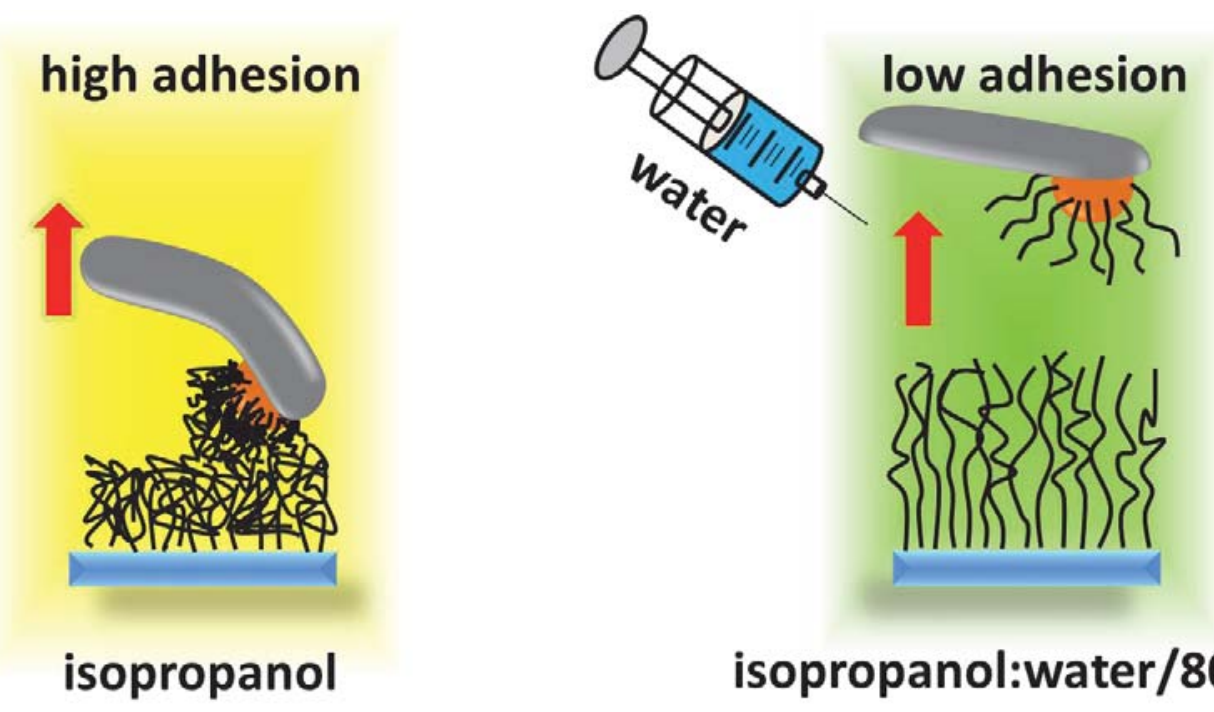

isopropanol:water/80:20

We present a simple method to reversibly switch the adhesive force between two surfaces that are decorated with poly(methyl methacrylate) (PMMA) graft polymers. By employment of a PMMA/isopropanol/water or a PMMA/ethanol/water cosolvent system, we can tune the swelling of the brushes. In pure isopropanol or ethanol the polymer grafts are collapsed, and the adhesion is high when the contacting brushes are pulled apart. In an 80-20 vol \% isopropanol-water or ethanol-water composition, the brushes are swollen. In these systems the adhesion is approximately 5 times smaller compared to the adhesion measured in the pure solvent systems. Moreover, we show that PMMA/isopropanol/water cosolvent systems perform better as switchable adhesives than PMMA/ethanol/water cosolvent systems. In the latter pulling events can arise when the swollen brushes are kept in contact for a longer time, such that the adhesion hysteresis can become large and the surface coating can be damaged due to bond-breaking events.

* This chapter has been published in: Yu, Y.; Kieviet, B. D.; Kutnyanszky, E.; Vancso, G. J.; de Beer, S., ACS Macro Lett. 2015, 4 (1), 75-79. 


\subsection{Introduction}

Controlling the magnitude of adhesion between two objects is of crucial importance for a wide variety of applications, which include: "pick up and place" systems, ${ }^{1}$ gecko or mussel inspired tapes, ${ }^{2}$ and biomedical glues. ${ }^{3}$ A versatile method of achieving such adhesion control is the functionalization of surfaces with polymeric materials, ${ }^{4}$ for example hydrogels, ${ }^{5}$ films, ${ }^{6}$ and especially brushes. ${ }^{7}$ These polymeric systems allow for reversible switching of the adhesive force between low and high values.

Under good solvent conditions, a polymer has an extended coil conformation, while in poor solvents, polymers are in a collapsed globule state. ${ }^{8}$ Switching between the coil and globule state can be achieved by changing the effective solvent quality, for example by directly replacing the solvent, ${ }^{9}$ by co-non-solvency, ${ }^{10}$ by UV light, ${ }^{11}$ by changing the temperature, ${ }^{12}$ by changing the oxidation state, ${ }^{13}$ or by adjusting the $\mathrm{pH}$ of the solvent. ${ }^{14}$ Upon endanchoring polymers to a surface at a sufficiently high density, a so-called polymer brush is formed. ${ }^{15}$ When such brushes are in good solvents, the polymers stretch away from the surface, whereas in poor solvents, they form a collapsed, dense film on the surface.

Swollen polymer brushes can act as efficient lubricants ${ }^{16}$ but can also find application in bioengineering ${ }^{17}$ or oil recovery. ${ }^{18}$ The adhesion between two contacting, brushfunctionalized surfaces depends on the conformational state of the polymers, e.g. whether the brushes are swollen or collapsed. The adhesion between two collapsed polymer brushes is generally high ${ }^{7}$ and determined by intermolecular interactions between the contacting polymers. In contrast, the adhesion is often observed to be low under good solvent conditions ${ }^{7}$ because the attractive polymer-polymer interactions are now overshadowed by more favorable polymer-solvent interactions such that the polymers effectively repel each other.

Keeping in mind potential applications of switchable adhesives, we anticipate that the necessity of a constant energy supply to maintain a constant high or low adhesion, as one would need for thermoresponsive polymers ${ }^{7}$ or polymers that change their conformation in an external electric field, ${ }^{19}$ would not be preferred. Also, removing components from the system (e.g., salt ${ }^{20}$ or one of the solvents in a solvent mixture ${ }^{21}$ ) to trigger switching from the default state would be suboptimal, while this removal is not always practically feasible. Ideally, one would like to have the default adhesive state in pure solvent, while addition of a component triggers the adhesion switch. For example, for a "pick up and place" system, the default state for pick up asks for a high adhesive force and thus poor solvent conditions in pure solvent. A component addition should trigger brush swelling resulting in low adhesion and therefore deposition of the picked up material. Triggering by cosolvency, in which a mixture of two poor solvents becomes a good solvent for a particular type of polymer, meets all demands described above for a "pick up and place" system. 
Nevertheless, cosolvency has, to our knowledge, not been explored in the context of smart surface coatings.

Cosolvency is a generic phenomenon that has been observed in a wide variety of polymers with different solvent mixtures ${ }^{22}$ and is commonly applied in the pharmaceutical industry and environmental engineering. ${ }^{23}$ The mechanism can be qualitatively understood from the Flory-Huggins theory ${ }^{24}$ using the single liquid approximation of Scott, ${ }^{25}$ where the effective Flory-Huggins parameter $\chi_{\mathrm{PSm}}$ between polymer P and solvent mixture Sm (of solvents $\mathrm{S} 1$ and S2 with volume fractions $\phi_{1}$ and $\left.\phi_{2}\right)$ is $\chi_{\mathrm{PSm}}=\phi_{1} \chi_{\mathrm{PS} 1}+\phi_{2} \chi_{\mathrm{PS} 2}-\phi_{1} \phi_{2} \chi_{\mathrm{S} 1 \mathrm{~S} 2}$. The effective interaction parameter $\chi_{\mathrm{PSm}}$ is the weighted average of the independent interactions $\chi_{\mathrm{PS} 1}$ and $\chi_{\mathrm{PS} 2}$, corrected for the reduction in interaction due to mixing of the two solvents.

When the two solvents are poor solvents for the polymer $\left(\chi_{\mathrm{PS} 1}\right.$ and $\chi_{\mathrm{PS} 2}>0.5$, in the limit of infinitely long chains $\left.{ }^{25}\right)$ and the solvents are miscible $\left(\chi_{\mathrm{S} 1 \mathrm{~S} 2}<2\right.$, within the regular solution $\left.\operatorname{model}^{25-26}\right) \chi_{\mathrm{PSm}}$ can be smaller than 0.5 such that the solvent mixture becomes a good solvent for the polymer. The single liquid approximation discussed above only provides a qualitative explanation for cosolvency. To quantitatively reproduce the ternary phase diagrams of polymers in solvent mixtures more detailed models are required. ${ }^{25,27}$ We note that the mechanism behind the globule-coil-globule transitions upon increasing the cosolvent content is different from the mechanism governing co-non-solvency. In the latter, the coil-globule transition in a mixture of good solvents in triggered by preferential absorbance of the better solvent on the polymer. ${ }^{28}$

In this article we show that adhesion between two opposing poly(methyl methacrylate) (PMMA) brushes can be reversibly switched from high to low using cosolvency of PMMA in isopropanol-water and ethanol-water mixtures. At room temperature, pure alcohols (such as isopropanol and ethanol) and pure water are considered to be poor solvents for PMMA, while mixtures prepared within a particular range of alcohol-water ratios form good solvents for PMMA. ${ }^{27 a}, 29$ Using atomic force microscopy (AFM), we determine the adhesive force between high density PMMA brushes that are grafted from a flat silicon surface and a gold colloid probe (of diameter $6 \mu \mathrm{m}$, spring constant $0.14 \mathrm{~N} / \mathrm{m}$ ) by surfaceinitiated atom transfer radical polymerization (grafting density 0.25 chains $/ \mathrm{nm}^{2}$ and degree of polymerization 8000) via force versus distance measurements. In the experiments, we monitor the deflection of the cantilever upon approach to and retract from the surface (distance $700 \mathrm{~nm}$ and velocity $420 \mathrm{~nm} / \mathrm{s}$ ). We find that the adhesion between PMMA brushes in an 80-20 vol \% mixture of isopropanol-water or ethanol-water is approximately a factor 5 lower than that for the brushes in pure isopropanol or ethanol. Therefore, our system would be suitable for "pick up and place" types of applications. Moreover, we show that the performance of the switchable adhesive is better in isopropanol-water mixtures than in ethanol-water mixtures. In the latter the effective interaction parameter between the polymers and the mixed solvents is higher than in the former, which results in only minor PMMA brush swelling and thus more direct polymer-polymer interaction in ethanol-water 
mixtures. Transient interdigitation ${ }^{44}$ allows for deeper penetration and thus a strong polymer-polymer interaction when the brushes are kept in contact for a longer time. As a consequence, we observe bond-breaking polymer-pulling events in the force-distance measurements such that the adhesion hysteresis per indentation cycle of PMMA brushes in an ethanol-water mixture can be up to a factor of 3 higher than in pure solvent.

\subsection{Materials and Methods}

\subsubsection{Materials}

Methyl methacrylate (MMA, 99\%) was passed through a basic alumina column to remove the inhibitor before use. Copper (I) bromide (CuBr, Aldrich, 98\%) was stirred in excessive acetic acid and filtered till the suspension solution is pale like, and dried in vacuum oven at

room temperature overnight. 11-[11-(2-bromo-2-methyl-propionyl-oxy)undecyldisulphanyl]-undecyl ester was synthesized according to a reported literature ${ }^{30} .2,2^{\prime}-$ Bipyridyl ( $\geq$ 99\%), (3-aminopropyl)triethoxysilane, 2-bromo-2-methylpropionyl bromide (98\%), triethylamine (TEA, $\geq 99 \%$ ), copper (II) bromide ( $\geq 99 \%$ ), Ethylenediaminetetraacetic acid tetrasodium salt dihydrate (EDTA, 99-102\%) were purchased from Sigma-Aldrich, and used as received without any purification. Methanol (absolute), 2-proponal (AR) and toluene (AR) were purchased from Biosolve. Hydrogen peroxide $\left(\mathrm{H}_{2} \mathrm{O}_{2}\right)$ and ethanol were purchased from Merck. Water from a MilliQ Advantage A 10 purification system (Millipore, Billerica, Ma, USA) was used.

\subsubsection{Preparation of polymer brushes}

We used surface initiated atom transfer radical polymerization (SI-ATRP) ${ }^{31}$ to graft PMMA brushes from silicon surfaces, gold surfaces and gold colloids connected to silicon AFM cantilevers (6 $\mu \mathrm{m}$ diameter, SQube, CP-CONT-Au, NanoAndMore, Spielburg, Germany). We chose the latter force probe to ensure that we graft the polymers from the gold colloid rather than the silicon cantilever.

\section{Polymerization on Gold}

In order to prove we can graft polymers on the gold colloids, the same experiments also were done on gold substrates for comparison. First, gold substrates and gold colloids were washed with chloroform and ethanol, then immersed in $1 \mathrm{mM}$ initiator 11-[11-(2-bromo-2methyl-propionyl-oxy)-undecyldisulphanyl]-undecyl ester solution in chloroform. After 12 $\mathrm{h}$, the substrates and gold colloids were rinsed in chloroform and ethanol, respectively. To achieve polymerization, the initiator-covered substrates and gold colloids cantilevers were placed in dry vials and purged with argon (Ar) for $1 \mathrm{~h}$. The monomer, MMA (10 g, 0.1 $\mathrm{mol})$, was dissolved in the ATRP medium (9 $\mathrm{ml}$ methanol/water mixture with volume ratio 5:1) and the solution was degassed for $2 \mathrm{~h}$. $\mathrm{CuBr}(145 \mathrm{mg}, 1 \mathrm{mmol})$ and 2,2'-bipyridine (320 mg, $2 \mathrm{mmol}$ ) were added to another flask equipped with a magnetic stirring bar, and 
deoxygenized by three vacuum-Ar backfill cycles. The degassed monomer solution was transferred into the flask and stirred under Ar for another $15 \mathrm{~min}$ until a clear brown solution was observed. Afterwards, the polymerization mixture was injected into each reaction vial, adding enough solution to submerge each sample completely. The polymerization was conducted for $18 \mathrm{~h}$ at room temperature, after which the samples and cantilevers were removed from the vials and washed with ethanol and chloroform through multiple cycles. Finally, the substrates were dried under a stream of nitrogen. The PMMA brush-modified colloid probes were kept in chloroform.

\section{Polymerization on Silicon}

The growth of PMMA brushes on silicon substrates is similar to the grafting method from gold substrates. However, for the initiator grafting, we use a two steps method. Silicon substrates were cut to small pieces from a round silicon sheet, and cleaned by Piranha solution $\left(\mathrm{H}_{2} \mathrm{SO}_{4}: \mathrm{H}_{2} \mathrm{O}_{2}=3: 1, \mathrm{v} / \mathrm{v}\right)$, then rinsed extensively with water, ethanol, and dried using nitrogen stream. Piranha solution reacts strongly with many organic compounds and should be handled with extreme caution. Dried substrates and (3-aminopropyl) triethoxysilane $(100 \mu \mathrm{L})$ were placed in a desiccator, after pumped by 15 minutes, vapor deposition was allowed to proceed overnight. Afterwards the silicon substrates were washed with ethanol and water sequentially, and dried in a nitrogen stream. In a beaker, a solution of toluene $(20 \mathrm{~mL})$ and TEA $(0.1 \mathrm{~mL})$ was prepared and cooled with ice to $0{ }^{\circ} \mathrm{C}$, and then the substrates were immersed into the solution with a sample holder (to avoid precipitated salt adhering on the substrates). 2-Bromo-2-methylpropionly bromide $(0.1 \mathrm{~mL})$ was added to the stirring solution drop by drop. The reaction was carried out for $1 \mathrm{~h}$ at room temperature, after which the substrates were rinsed with toluene, and sonicated for $1 \mathrm{~min}$. Then the substrates were washed with ethanol and water, and dried under a stream of nitrogen. The polymerization process is the same with that on gold substrate. At last, the silicon substrates are rinsed in EDTA solution overnight to remove the copper, and dried with nitrogen.

\subsubsection{Characterization}

\section{Fourier Transform Infrared (FTIR)}

FTIR spectroscopy was employed to establish which groups are present in the tethered brushes after substrate anchoring. FTIR spectra were obtained using a Bruker Vertex 70v spectrometer (beam splitter: KBr, 1024 scans). A background spectrum was first obtained by scanning a clean silicon or gold substrate. The obtained spectrum of PMMA brushes on gold substrate is shown in Figure 6.1. 3050-2990 $\mathrm{cm}^{-1}$ (CH stretching vibration), 1730 $\left(\mathrm{C}=\mathrm{O}\right.$ double bond stretching vibration), $1450\left(\mathrm{CH}_{3}, \mathrm{CH}_{2}\right.$ deformation vibration), 12601040 (C-O-C stretching vibration), 880-960 (C-O-C deformation vibration). The characteristic group of PMMA is $\mathrm{C}=\mathrm{O}$ appeared at $1730 \mathrm{~cm}^{-1}$, which proves the success of grafting polymers on substrates and cantilevers. 


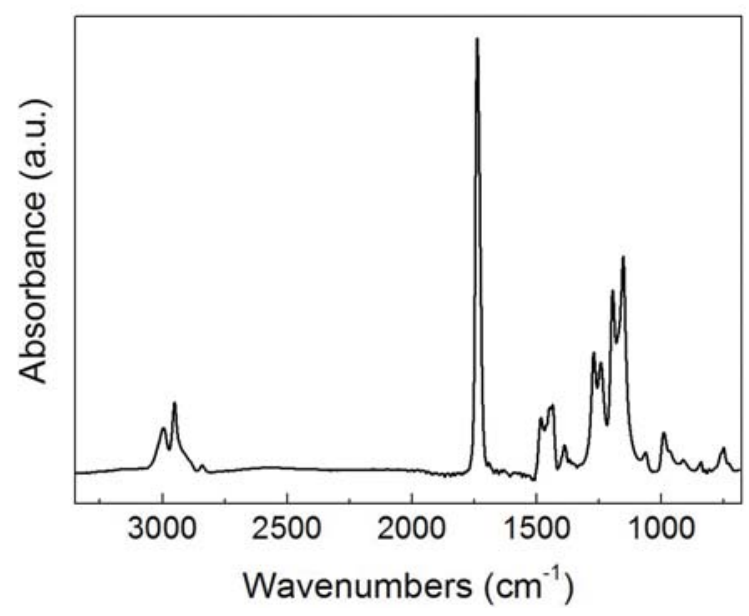

Figure 6.1. FTIR spectrum of PMMA brushes on gold substrate.

\section{Atomic Force Microscopy (AFM)}

The AFM measurements were performed on a Multimode 8 AFM using a NanoScope V controller, a JV vertical engage scanner and a liquid cell (Bruker, San Barbara, CA). Gold colloids connected to silicon cantilevers (6 $\mu \mathrm{m}$ diameter, sQube, NanoAndMore, CPCONT-Au, Germany) were used. Non-coated cantilevers are used in all the measurements, because they are less sensitive to changes in the surroundings such that we can exchange the solvents back and forth without needing to realign the laser. The spring constant (typically $k=0.137 \mathrm{~N} / \mathrm{m} \pm 0.003$ ) is determined in air using the thermal noise method $^{32}$ as implemented in the Nanoscope 8.15 software. In the experiments we monitor the static deflection of the cantilever upon approach to and retract from surface over $700 \mathrm{~nm}$ with velocity $v=420 \mathrm{~nm} / \mathrm{s}$. During the approach the cantilever is moved towards the surface until the force is typically $12.3 \mathrm{nN} \pm 1.7$. The adhesion we define as the force needed to pull the contacting brushes apart upon retract of the cantilever. We determined the adhesive force in pure and mixed solvents, which were successively penetrated into liquid cell (4 times). After each measurement, the surface and the liquid cell were dried by a gentle supply of nitrogen, after which everything is remounted into optical head of AFM. The adhesion hysteresis was calculated by integrating the difference in force upon approach and retract as a function of the piezo displacement. We determined the number of pulling events as a function of the delay time in various solvents by counting the number of local forceminima in the force versus piezo displacement curve monitored upon retracting the cantilever. 


\subsection{Results and Discussion}

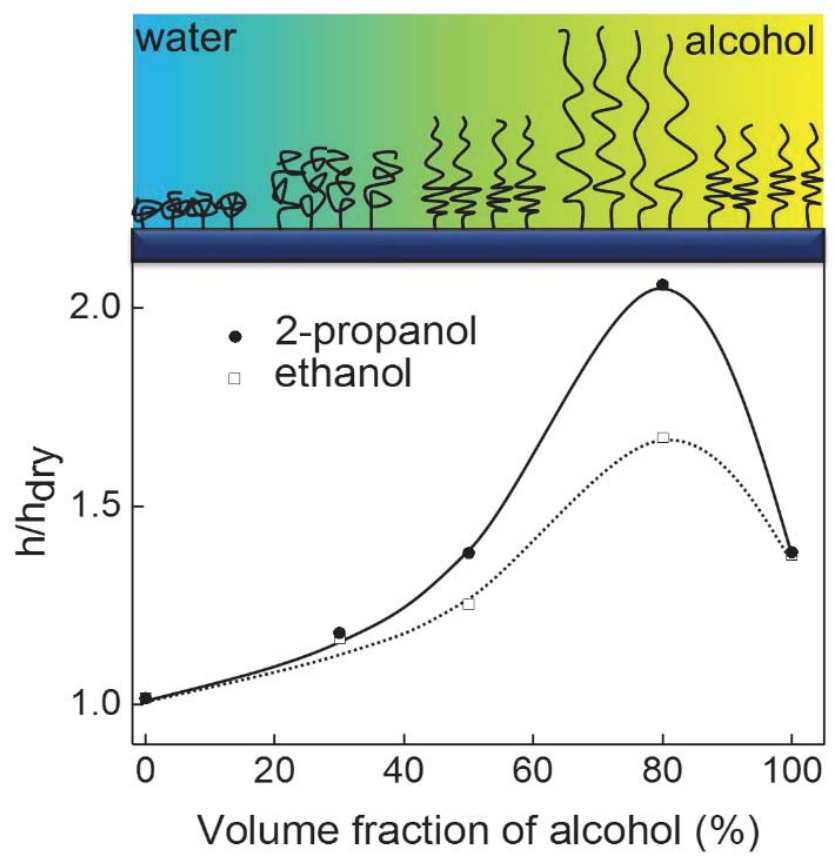

Figure 6.2. Height $\mathrm{h}$ of the PMMA brush on the flat surface for various alcohol-water ratios (isopropanol closed symbols and ethanol open symbols) as measured by AFM using a small normal load (of $1 \mathrm{nN}$ ). The measured height is normalized by the height of the brush measured under dry conditions $\left(\mathrm{h}_{\mathrm{dry}}\right)$. The lines are guides to the eye.

Figure 6.2 shows the swelling ratio of the PMMA brush as determined from AFM images captured under low normal loads $(1 \mathrm{nN})$ in a region where, prior to the experiments, part of the brush was gently removed from the surface by scratching using Teflon tweezers. More details on the experimental procedures, sample preparation, and characterization can be found in materials and methods part. As the alcohol (isopropanol or ethanol) content of the solvent mixture is increased, the brush height increases. The brush height was found to be maximum for the $80-20$ vol \% alcohol-water mixtures and decreases as the alcohol content is further increased above $80 \%$. In pure water the brush swells by approximately $2 \%$, which is consistent with the low absorption of water (4.5 wt \%) in bulk PMMA. ${ }^{33}$ In pure isopropanol the brush swells by $38 \%$, which is close to the $46 \mathrm{wt} \%$ of isopropanol absorbed in bulk PMMA, measured using a Cahn counterbalance. ${ }^{34}$ Though the isopropanol absorbance in PMMA is significant, isopropanol is still considered to be a poor solvent for PMMA because the upper critical solution temperature (UCST) is above room temperature $(350 \mathrm{~K}){ }^{29 \mathrm{a}}$

The height of the brush solvated in pure ethanol is only slightly smaller than the height measured in isopropanol $(<1 \%)$, which is in qualitative agreement with the UCST of ethanol/PMMA being only $5 \mathrm{~K}$ higher than the UCST of isopropanol/PMMA. ${ }^{29 \mathrm{~b}}$ The 
stronger affinity of the alcohols with PMMA compared to water shifts the solubility maximum toward high relative alcohol concentrations and is typically found at an 80-20 vol. $\%$ alcohol-water mixture. ${ }^{27 a}$, 29 At the solubility maximum, the brush swells by $67 \%$ in the ethanol-water mixture, while it swells by more than a factor of $2(105 \%)$ in isopropanolwater. The reason for this strong difference in brush swelling for the different alcohols is the slightly smaller interaction between ethanol/PMMA compared to isopropanol/PMMA. ${ }^{29 \mathrm{~b}}$ Due to these reduced interactions, $\chi_{\mathrm{PSm}}$ will be a bit smaller for isopropanol/water/PMMA than for ethanol/water/PMMA, which can result in relatively large changes in the brush height when $\chi_{\mathrm{PSm}}$ is close to the $\theta$-point ${ }^{35}$ (of $\chi=0.5$ for infinitely long chains ${ }^{25}$ ). The latter implies that a mixture of water and ethanol or isopropanol becomes a mediocre, but not a good, solvent for PMMA. ${ }^{36}$ In fact, in acetophenon (good solvent for PMMA) our swelling ratio was measured to be much higher (4.3).

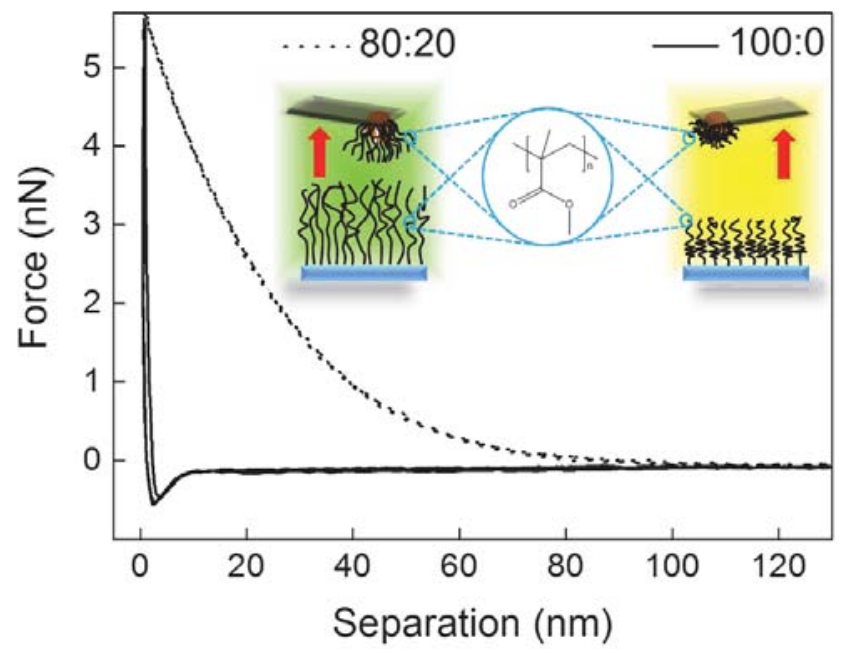

Figure 6.3. Typical force curves upon retract of the AFM cantilever from the surface in the 80-20 vol.\% isopropanol-water mixture (three curves, dashed lines) and in 100\% isopropanol (three curves, solid lines).

Figure 6.3 shows three typical force versus tip-surface-separation curves upon retract of the PMMA surfaces using either pure isopropanol as a solvent (solid lines) or an 80-20 vol.\% isopropanol-water solvent mixture (dashed lines). The "zero" in Figure 6.3 is set by the distance at which the slope of the force curve goes to infinity, which happens when the elastic repulsion stiffness is much stronger than the stiffness of the cantilever and should not be confused with colloid-surface contact. The difference between the force traces measured under the same conditions is small $(<10 \%$, see Figure 6.3$)$.

However, the force traces measured in pure isopropanol or in the mixture are qualitatively different (dashed lines versus full lines in Figure 6.3). For the swollen brushes (80-20 vol.\% mixture), we observe a long-range repulsive interaction, due to the osmotic pressure in the 
solvent. $^{37}$ The adhesion hysteresis is small (not shown) and determined by the hydrodynamic resistance of the solvent moving through the brushes. ${ }^{37 b,} 38$ Due to this hysteresis, there is a small attractive (adhesive) force $(<0.1 \mathrm{nN})$ upon retract of the surfaces, which would disappear in the limit of retract-velocity $\mathrm{v} \rightarrow 0$. However, this limit is difficult to reach experimentally due to noise and drift limitations. For the collapsed brushes in pure isopropanol, the force upon retraction is determined by attractive polymer-polymer interactions. Consequently, we observe a clear adhesive force of more than $0.4 \mathrm{nN}$.

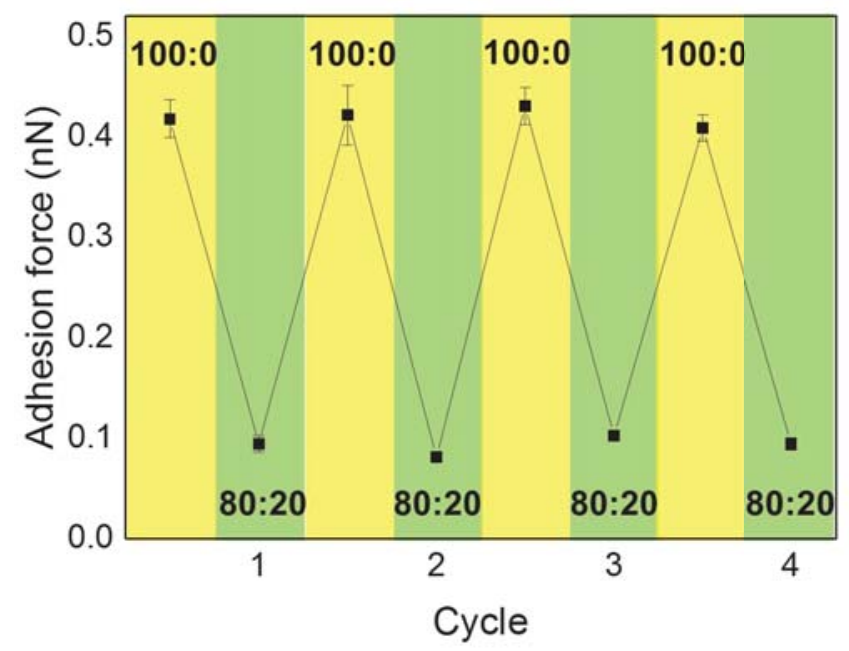

Figure 6.4. Adhesive force between the PMMA brushes after solvent exchange from 100-0 vol. $\%$ isopropanol-water to an $80-20$ vol.\% isopropanol-water mixture. The adhesion is typically 5 times higher in pure isopropanol than in the 80-20 solvent mixture. The error bars denote the standard error of the mean with a $95 \%$ confidence interval.

Figure 6.4 shows the adhesive force between the PMMA brushes covered surfaces, averaged over typically 20 force-distance curves captured on the same position on the sample surface, for four consecutive solvent exchanges from pure isopropanol to the 80-20 vol.\% isopropanol-water mixture and back. The adhesive force measured under poor solvent conditions is consistently 5 times higher than the adhesive force between the swollen brushes. The variation in the adhesive force between different cycles is $<10 \%$ and is most likely caused by small differences in the solvent composition that can occur during evaporation of the solvent. Experiments performed in pure ethanol and an 80-20 vol.\% ethanol-water mixture show qualitatively the same results, except that we observed pulling events in approximately $40 \%$ of the force-distance curves obtained in the ethanol-water mixture. Such pulling events occur when bonds are broken during retraction of the two surfaces. ${ }^{39}$ For our system we attribute bond breaking to an increase in polymer-polymer interactions due to the less favorable interaction of PMMA with ethanol-water compared to 
isopropanol-water. The number of pulling events strongly depends on the time the surfaces are kept in contact.

Figure 6.5. (a) shows typical force versus sample displacement curves obtained upon retract of the PMMA brushes in an 80-20 vol.\% ethanol-water mixture for no delay time (dashed lines) and $10 \mathrm{~s}$ delay time (full lines). The delay time is the extra time that the surfaces are kept in contact at the maximum in the normal force, on top of the time that the surfaces are already in contact (typically $0.25 \mathrm{~s}$ ) during a default approach-retract cycle. Figure 6.5. (a) clearly shows that the number of pulling events $N$ increases with the delay time. Figure 6.5 . (b) shows how $N$ evolves as the delay time is increased. For the 80-20 vol.\% ethanol-water mixture $N$ increases approximately exponentially from 0.4 at $0 \mathrm{~s}$ to 6 at $10 \mathrm{~s}$ delay time. The shape of the exponential suggests that there are several relaxation processes during contact formation, as was observed before by others via monitoring the density profile of a dry polystyrene brush that is brought into contact with a polystyrene network. ${ }^{40}$
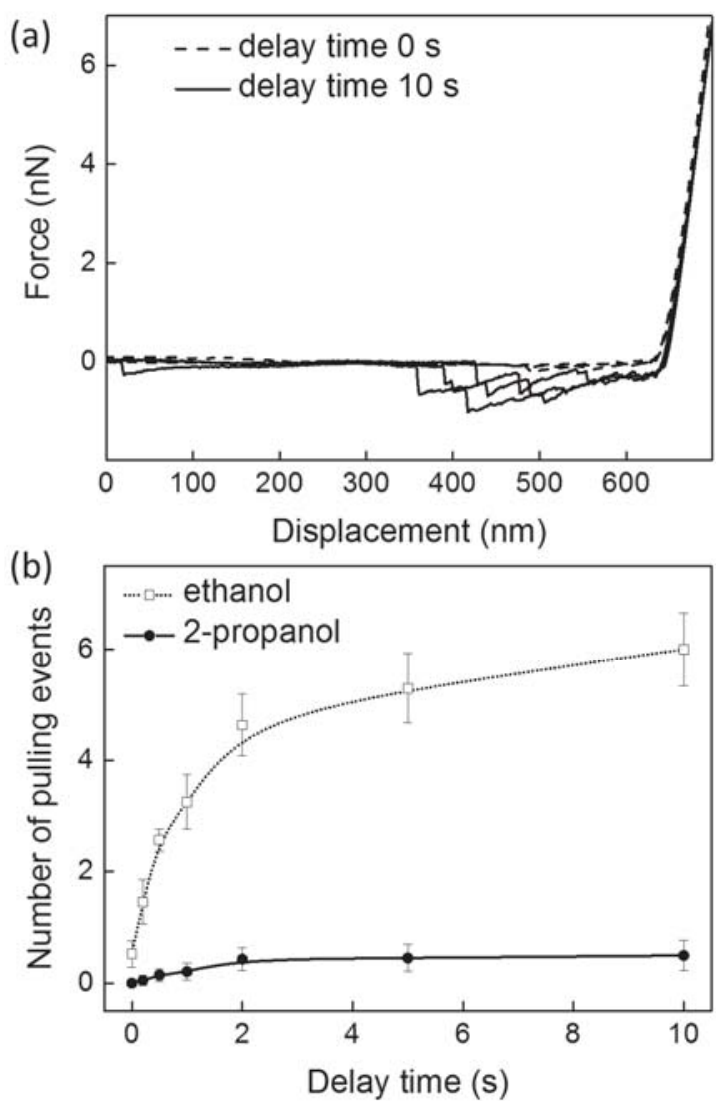

Figure 6.5. (a) Force versus displacement curves that are obtained upon retracting the cantilever directly or after a delay time of $10 \mathrm{~s}$ from the surface. The PMMA brushes are solvated in an 80-20 vol.\% ethanol-water mixture. (b) The evolution of the number of pulling events as a function of the delay time for an 80-20 vol.\% ethanol-water mixture (open symbols) and for an 80-20 vol.\% isopropanol-water mixture (closed symbols). The error bars denote the standard error of the mean with a $95 \%$ confidence interval. 
For the $80-20$ vol.\% isopropanol-water mixture $N$ increases to only 0.5 after a $10 \mathrm{~s}$ delay time, which is much less than for the experiments in ethanol-water mixtures. We attribute this difference to the different effective solvent qualities for PMMA that are created by the ethanol-water and the isopropanol-water mixtures. The isopropanol-water mixture is a better solvent for PMMA than the ethanol-water mixture, such that there is more solvent absorbed into the brush using the isopropanol-water mixture (see Figure 6.2). This makes the effective PMMA-PMMA interaction smaller. As a consequence, one would expect that the number of pulling events is higher for measurements in pure solvents, where there is even less solvent absorbed in the brush. However, a higher polymer density also implies that the relaxation time of interdigitation increases (up to 3-4 h for dry brushes ${ }^{40}$ ) such that the polymers do not have enough time to interdigitate during the experiment. For the PMMA/ethanol/water cosolvent system, there is just the right amount of solvent absorbance to induce bond-breaking pulling events that can be measured on the time scale of the experiment. These results are in agreement with the observation that strong permanent adhesives can be formed by cross-linking PMMA films that are swollen in ethanol-water mixtures. ${ }^{41}$ Since pulling events can significantly increase the adhesion hysteresis after longer contact times and because bond-breaking events will damage the surface coating, we propose that the PMMA/isopropanol/water system is a better candidate for the development of a switchable adhesive than the PMMA/ethanol/water system.

\subsection{Conclusions}

In summary, we have shown that the adhesion between two opposing brushes can be switched from high to low and back using cosolvency of PMMA in isopropanol-water and ethanol-water mixtures. The adhesive force between PMMA brushes in pure isopropanol or ethanol was found to be a factor of 5 higher than in an 80-20 vol.\% alcohol-water mixture. The system in pure solvent will show high adhesion, while low adhesion can be triggered by addition of the cosolvent. Therefore, our technique is very suitable for "pick up and place" type of applications. Moreover, we showed that switchable adhesion can best be obtained in isopropanol-water mixtures instead of ethanol-water mixtures, due to bondbreaking pulling events that can occur in the latter system. 


\subsection{References}

[1] M. J. Vogel and P. H. Steen, Proceedings of the National Academy of Sciences of the United States of America 2010, 107, 3377-3381.

[2] H. Lee, B. P. Lee and P. B. Messersmith, Nature 2007, 448, 338-342.

[3] A. Meddahi-Pellé, A. Legrand, A. Marcellan, L. Louedec, D. Letourneur and L. Leibler, Angewandte Chemie 2014, 126, 6487-6491.

[4] M. A. C. Stuart, W. T. S. Huck, J. Genzer, M. Muller, C. Ober, M. Stamm, G. B. Sukhorukov, I. Szleifer, V. V. Tsukruk, M. Urban, F. Winnik, S. Zauscher, I. Luzinov and S. Minko, Nature Materials 2010, 9, 101113.

[5] a) D. P. Chang, J. E. Dolbow and S. Zauscher, Langmuir 2007, 23, 250-257; b) S. Schmidt, M. Zeiser, T. Hellweg, C. Duschl, A. Fery and H. Mohwald, Advanced Functional Materials 2010, 20, 3235-3243.

[6] a) A. Dedinaite, E. Thormann, G. Olanya, P. M. Claesson, B. Nystrom, A. L. Kjoniksen and K. Z. Zhu, Soft Matter 2010, 6, 2489-2498; b) X. Feng, B. D. Kieviet, J. Song, P. M. Schön and G. J. Vancso, Applied surface science 2014, 292, 107-110.

[7] a) D. M. Jones, J. R. Smith, W. T. S. Huck and C. Alexander, Advanced Materials 2002, 14, 1130-1134; b) E. M. Benetti, S. Zapotoczny and J. Vancso, Advanced Materials 2007, 19, 268-271; c) I. B. Malham and L. Bureau, Langmuir 2010, 26, 4762-4768.

[8] P. J. Flory, Principles of polymer chemistry, Cornell University Press, 1953, p.

[9] P. Auroy and L. Auvray, Macromolecules 1992, 25, 4134-4141.

[10] a) F. M. Winnik, H. Ringsdorf and J. Venzmer, Macromolecules 1990, 23, 2415-2416; b) D. Mukherji and K. Kremer, Macromolecules 2013, 46, 9158-9163.

[11] M. Irie, A. Menju and K. Hayashi, Macromolecules 1979, 12, 1176-1180.

[12] S. Uenoyama and A. S. Hoffman, International Journal of Radiation Applications and Instrumentation. Part C. Radiation Physics and Chemistry 1988, 32, 605-608.

[13] X. Sui, X. Feng, J. Song, M. A. Hempenius and G. J. Vancso, Journal of materials chemistry 2012, 22, 11261-11267.

[14] T. Wu, P. Gong, I. Szleifer, P. Vlc`ek, V. Šubr and J. Genzer, Macromolecules 2007, 40, 8756-8764.

[15] a) S. Alexander, Journal De Physique 1977, 38, 983-987; b) P. de Gennes, Macromolecules 1980, 13, 1069-1075.

[16] a) S. Lee and N. D. Spencer, SCIENCE-NEW YORK THEN WASHINGTON- 2008, 319, 575; b) M. Chen, W. H. Briscoe, S. P. Armes and J. Klein, science 2009, 323, 1698-1701; c) S. de Beer, E. Kutnyanszky, P. M. Schon, G. J. Vancso and M. H. Muser, Nature Communications 2014, 5, 3781.

[17] P. M. Mendes, Chemical Society Reviews 2008, 37, 2512-2529.

[18] J. Stanislav in Use of polymers in oil recovery processes, Springer, 1982, pp. 210-211.

[19] C. Drummond, Physical review letters 2012, 109, 154302.

[20] Q. B. Wei, M. R. Cai, F. Zhou and W. M. Liu, Macromolecules 2013, 46, 9368-9379.

[21] Q. Chen, E. S. Kooij, X. F. Sui, C. J. Padberg, M. A. Hempenius, P. M. Schon and G. J. Vancso, Soft Matter 2014, 10, 3134-3142.

[22] a) L. Cragg and H. Hammerschlag, Chemical Reviews 1946, 39, 79-135; b) C. Bamford and H. Tompa, Transactions of the Faraday Society 1950, 46, 310-316; c) P. J. Flory and W. R. Krigbaum, Annual review of physical chemistry 1951, 2, 383-402; d) B. Wolf and G. Blaum, Journal of Polymer Science: Polymer Physics Edition 1975, 13, 1115-1132.

[23] G. Wypych, Handbook of solvents, ChemTec Publishing, 2001, p.

[24] a) P. J. Flory, The Journal of chemical physics 1942, 10, 51-61; b) M. L. Huggins, Annals of the New York Academy of Sciences 1942, 43, 1-32.

[25] R. L. Scott, The Journal of Chemical Physics 1949, 17, 268-279.

[26] P. M. Chaikin and T. C. Lubensky, Principles of condensed matter physics, Cambridge Univ Press, 2000, p.

[27] a) S. M. Lee and Y. C. Bae, Polymer 2014, 55, 4684-4692; b) T.-H. Young, L.-P. Cheng, C.-C. Hsieh and L.-W. Chen, Macromolecules 1998, 31, 1229-1235.

[28] D. Mukherji, C. M. Marques and K. Kremer, Nature communications 2014, 5, 4882.

[29] a) J. M. Cowie, M. A. Mohsin and I. J. McEwen, Polymer 1987, 28, 1569-1572; b) R. Hoogenboom, C. R. Becer, C. Guerrero-Sanchez, S. Hoeppener and U. S. Schubert, Australian journal of chemistry 2010, 63, 1173-1178.

[30] K. Matyjaszewski, P. J. Miller, N. Shukla, B. Immaraporn, A. Gelman, B. B. Luokala, T. M. Siclovan, G. Kickelbick, T. Vallant and H. Hoffmann, Macromolecules 1999, 32, 8716-8724.

[31] X. F. Sui, Q. Chen, M. A. Hempenius and G. J. Vancso, Small 2011, 7, 1440-1447.

[32] J. L. Hutter and J. Bechhoefer, Review of Scientific Instruments 1993, 64, 1868-1873. 
[33] J. Y. Lai, S. F. Lin, F. C. Lin and D. M. Wang, Journal of Polymer Science Part B: Polymer Physics 1998, 36, 607-615.

[34] L. P. Cheng and H. Y. Shaw, Journal of Polymer Science Part B: Polymer Physics 2000, 38, 747-754.

[35] K. Binder, T. Kreer and A. Milchev, Soft Matter 2011, 7, 7159-7172.

[36] D. Mukherji, C. M. Marques, T. Stuehn and K. Kremer, The Journal of chemical physics 2015, 142, 114903.

[37] a) S. T. Milner, T. A. Witten and M. E. Cates, Macromolecules 1988, 21, 2610-2619; b) J. Klein, Y. Kamiyama, H. Yoshizawa, J. N. Israelachvili, G. H. Fredrickson, P. Pincus and L. J. Fetters, Macromolecules 1993, 26, 5552-5560.

[38] P. Nommensen, M. Duits, D. Van Den Ende and J. Mellema, Physical Review E 1999, 59, 3147.

[39] a) M. Rief, M. Gautel, F. Oesterhelt, J. M. Fernandez and H. E. Gaub, science 1997, 276, 1109-1112; b) H. Schönherr, M. W. Beulen, J. Bügler, J. Huskens, F. C. van Veggel, D. N. Reinhoudt and G. J. Vancso, Journal of the American Chemical Society 2000, 122, 4963-4967.

[40] M. Geoghegan, C. Clarke, F. Boué, A. Menelle, T. Russ and D. Bucknall, Macromolecules 1999, 32, 5106-5114.

[41] H. H. Tran, W. Wu and N. Y. Lee, Sensors and Actuators B: Chemical 2013, 181, 955-962. 



\section{Chapter 7}

\section{Substantially Enhanced Stability Against Degrafting of Zwitterionic Brushes by Utilizing PGMA-based Macro-initiators}

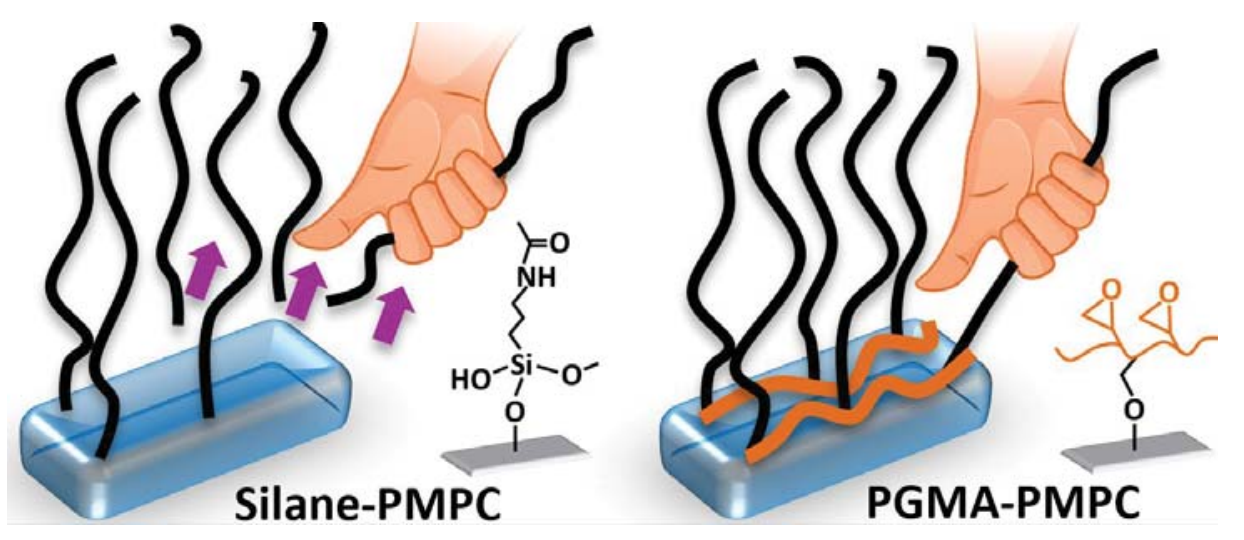

We present a simple method to prepare zwitterionic poly(2-methacryloyloxyethyl phosphorylcholine) (PMPC) brushes on silicon surfaces that exhibit excellent long term stability in aqueous environment. First, we attach poly(glycidyl methacrylate) (PGMA) to the substrate. Next, we couple 2-bromoisobutyryl bromide initiators to the modified substrate, which allows us to grow PMPC brushes using surface initiated atom transfer radical polymerization. We demonstrate that the PGMA-PMPC brushes are much more robust than the PMPC brushes grafted from commonly utilized silane-coupled initiators, for which a strong, ion-specific degrafting in aqueous media is observed. Atomic force microscopy (AFM) is employed to evaluate the decrease in dry thickness of the PMPC brushes after incubation in aqueous environments. The AFM results show that for the PGMA-PMPC brushes degrafting is indeed inhibited and that the brushes can stand being immersed for at least 4 weeks in physiological fluids (saline solution) and artificial sea water with only $1 \%$ decrease of their dry thickness. 


\subsection{Introduction}

Triggered by the recent increase in the demand of functional surface coatings, ${ }^{1}$ there has been a quickly growing scientific interest in polymer brushes. ${ }^{2}$ These brushes are dense films of macromolecules that are attached with one end to a surface. Surfaces decorated with brushes can be employed for controlling surface wettability, ${ }^{3}$ adhesion ${ }^{2 b}, 4$ and friction, ${ }^{5}$ and can be utilized in sensing ${ }^{6}$ and drug delivery, ${ }^{7}$ among others. In particular, zwitterionic polymer brushes, which bear both cationic and anionic groups in the same polymer chain, have drawn considerable attention, due to their excellent performance as lubricants ${ }^{8}$ and low-fouling coatings. ${ }^{9}$ Moreover, they are often bio-compatible, ${ }^{10}$ which makes them potential candidates for applications in the human body, for example on the interface of artificial joints ${ }^{10 b, 11}$ and on catheters. ${ }^{12}$

A well-studied example of a zwitterionic polymer is poly(2-methacryloyloxyethyl phosphorylcholine) (PMPC). Brushes composed of such polymers show extremely low sliding friction, which is even lower than typical values found in human synovial joints. ${ }^{8 a} \mathrm{~A}$ possible reason for this low frictional response is that water molecules can cooperatively bind with the zwitterionic moieties in the brushes resulting in a strong hydration. ${ }^{13}$ As a consequence, the osmotic pressure in the polymer brush system and thus the repulsion between opposing brushes is also high and the effective contact area is reduced, e.g. friction is lower during single asperity sliding. Therefore, these brushes hold great potential for employment in devices or machines where low friction is required. However, a critical disadvantage, which has prevented application of the brushes, is that the polymers can be cleaved off the surface during long-term immersion in aqueous solutions. ${ }^{14}$

The degrafting of PMPC brushes and other brushes is in most cases caused by hydrolysis, which can be tension-induced or -enhanced and results in breaking of the bonds between the polymer and the grafting surface. ${ }^{15}$ Strongly hydrated polymer brushes such as PMPC where the osmotic pressure and the stretching-induced tension is high ${ }^{14 \mathrm{~b}}$ are particularly sensitive to cleavage due to a better access of water to the hydrolysis sensitive bonds, but also due to the potential mechano-chemical shift in the hydrolysis equilibrium. In acidic and alkaline solutions, ${ }^{16}$ the cleavage of particular bonds can be accelerated even more. Which bond will break depends on the type of surface in combination with the type of anchor or polymerization-initiator used to prepare the brushes. ${ }^{15 b,} 17$ It was shown using molecular dynamics simulations that the highest tension occurs at the bond closest to the surface. ${ }^{18}$ This bond is, therefore, often considered to be the most likely candidate for breakage. However, other bonds in the brush-anchors, such as the common ester or amide bonds, can be sensitive to hydrolysis too. ${ }^{17 a}, 19$ Besides hydrolysis, other causes for degrafting can be trace amounts of HF formed in the solvent, which can partly etch the silicon-oxide grafting-surface ${ }^{17 c}$ or the utilization of light-sensitive linkers between the polymer and the surface. ${ }^{20}$ 
In the last years, many methods have been developed to slow down degrafting of brushes from the surfaces. ${ }^{14 a, 14 b, 17 a, 21}$ Examples include the use of dense bottle-brushes where the backbone attaches to the surface ${ }^{22}$ and the employment of long hydrophobic alkyl-chains that reduce hydrolytic activity by preventing water molecules from reaching the surfacebond. ${ }^{23}$ Alternatively, self-healing polymer coatings can be used to counter-act brush degradation by the polymer-reattachment. ${ }^{21 a}$ In this article, we build on these developments, as well as on our expertise in this area. ${ }^{14 a}, 24$ In a recent article, we have for example shown that the degrafting of neutral, water-soluble brushes is significantly slowed down by utilizing trimethoxysilane-based instead of monochlorosilane-based surface-initiated atom transfer radical polymerization (SI-ATRP) initiators. ${ }^{24}$ The former silane can bind to the surrounding silanes and the surface creating more anchor-points. Moreover, an amide bond in the trimethoxysilane replaces the ester-bond in the monochlorosilane. The latter bond is slightly more hydrolysis-sensitive (at extreme $\mathrm{pH}$ and temperature) than the amide. ${ }^{25}$ The method of Ref., ${ }^{24}$ however, only works for neutral polymers. For more hydrophilic, charged or zwitterionic polymers such as PMPC, the brushes still detach. ${ }^{14 a}, 14 b, 26$ For such very hydrophilic polymers, diblock copolymers systems have been proposed to obtain stable surface-coatings. ${ }^{14 a, 21 c, 27}$ Yet, under particular solvent conditions these coatings can phaseseparate creating a rough, heterogeneous surface. ${ }^{14 \mathrm{a}}$

In this article, we present a simple method to fabricate homogeneous and robust PMPC brushes, which show a substantial reduction in degrafting kinetics in water and salt solutions. To do so, we modify the poly (glycidyl methacrylate) (PGMA)-macro-initiator method first reported by Liu et al., ${ }^{28}$ where bromoacetic acid (BAA) initiators or 2-bromo2-methylpropionic acid (BPA) ${ }^{29}$ are coupled to the free epoxy groups on the silicon-oxidebound PGMA molecule. Instead, we couple 2-bromoisobutyryl bromide (BIBB) initiators to the hydroxyl group that is formed when PGMA epoxy-groups react with hydroxyl groups on the silicon surface. This leaves the non-reacted epoxy groups intact and, thus, the hydrophobic nature of the PGMA is maintained. This reduces the chance that water molecules reach the hydrolysis-sensitive surface bonds. Our prepared PGMA-PMPC brushes show an excellent degrafting-resistance in both water and aqueous salt media: The height of the PMPC brushes reduces by less than $1 \%$ after immersion in physiological (saline) and marine (0.6 M sodium chloride) environments for 4 weeks. Our brushes, therefore, hold great potential for application in e.g. biomedical engineering. 


\subsection{Materials and Methods}

\subsubsection{Materials}

Azobisisobutyronitrile (AIBN, Aldrich, 98\%) was recrystallized twice in methanol, and dried in the oven for $12 \mathrm{~h}$ at room temperature. Then it was kept in an amber flask, and stored in the fridge. Copper (I) bromide ( $\mathrm{CuBr}$, Aldrich, 98\%) was purified by stirring in acetic acid and filtered till the suspension solution was pale like. After washing with ethanol, the pale powder was dried in a vacuum oven at room temperature overnight. Poly (glycidyl methacrylate) (PGMA, $M_{n}=1 \times 10^{4}$ ), (3-aminopropyl)triethoxysilane (APTES), 2-Methacryloyloxyethyl phosphorylcholine (MPC, 97\%), 2,2'-Bipyridyl ( $\geq$ 99\%), triethylamine (TEA, $\geq 99 \%$ ), 2-bromo-2-methylpropionyl bromide (BiBB, 98\%), copper (II) bromide $\left(\mathrm{CuBr}_{2}, \geq 99 \%\right)$, sodium fluoride $(\mathrm{NaF}, \geq 99 \%)$, sodium chloride $(\mathrm{NaCl}, \geq 99 \%)$, sodium iodide ( $\mathrm{NaI}, \geq 99.5 \%), \mathrm{CDCl}_{3}\left(99.8\right.$ atom\% D) and $\mathrm{D}_{2} \mathrm{O}(99.9$ atom\% D) were purchased from Sigma-Aldrich, and used as received without any purification. Methanol (absolute), sulfuric acid (95-97\%), dimethylformamide (DMF), 2-proponal (AR) and toluene (AR) were purchased from Biosolve. Hydrogen peroxide $\left(\mathrm{H}_{2} \mathrm{O}_{2}\right)$ was purchased from Merck. MilliQ water was made from a MilliQ Advantage A 10 purification system (Millipore, Billerica, Ma, USA).

\subsubsection{Methods}

\section{Method 1: Synthesis of Silane-PMPC}

First, cut silicon substrates (approximately $1 \times 1 \mathrm{~cm}^{2}$ ) were cleaned by Piranha solution $\left(\mathrm{H}_{2} \mathrm{SO}_{4}: \mathrm{H}_{2} \mathrm{O}_{2}=3: 1, \mathrm{v} / \mathrm{v}\right)$, extensively rinsed with water and ethanol, and dried by flushing with nitrogen. Then vapor deposition of APTES $(0.1 \mathrm{~mL})$ on the substrates was conducted in a desiccator under vacuum for one night. Afterwards the initiator was grafted by immersing the surfaces in a mixture of triethylamine $(0.1 \mathrm{~mL})$ and 2-bromo-2methylpropionly bromide $(0.1 \mathrm{~mL})$ in toluene $(30 \mathrm{~mL})$ for $1 \mathrm{~h}$. (The substrates were placed perpendicularly standing on a sample holder to avoid precipitated salt adhering on the substrates). After the reaction, the substrates were sonicated in toluene for $1 \mathrm{~min}$, then washed with ethanol and water, and blown dry with nitrogen gas.

We used atom transfer radical polymerization to grow the brushes from the surface ${ }^{30}$. In a round bottom flask, MPC ( $2 \mathrm{~g}, 6.8 \mathrm{mmol})$ and 2,2'-bipyridyl (50 mg, $0.3 \mathrm{mmol})$ were dissolved in a MilliQ water $(2 \mathrm{~mL})$ and methanol $(2 \mathrm{~mL})$ mixture, and purged with argon for $15 \mathrm{~min}$. Meanwhile, $\mathrm{CuBr}(16.2 \mathrm{mg}, 0.1 \mathrm{mmol})$ and $\mathrm{CuBr}_{2}(7.5 \mathrm{mg}, 0.03 \mathrm{mmol})$ were also flushed with argon in another flask for 15 minutes. After that, the monomer solution was combined with the catalyst and bubbled with argon for another 15 minutes. Prior to the polymerization, the initiator-modified silicon substrates were flushed with argon in Erlenmeyer flask for at least 20 minutes. Later, the mixture combining monomer, ligand, and catalyst was transferred into the flask with the substrates. The polymerization was 
allowed to proceed for 4 hours at room temperature under argon atmosphere. $30 \mathrm{~mL}$ MilliQ water was injected into the flask to stop the reaction. Finally, the substrates were rinsed in water and ethanol solution to remove the physically adsorbed polymer.

\section{Method 2. Synthesis of PGMA-PMPC}

Firstly, the silicon substrate surfaces were activated with Piranha solution. Then the PGMA thin film was deposited on the cleaned substrates by dip coating in a concentration of $0.1 \%$ of PGMA in MEK solution, and dried in air. As reported before, increasing the aging time before annealing improved the thickness of PGMA film on the substrates. Here all the samples were kept in petri-dish for the aging time of $48 \mathrm{~h}$, then annealed for $30 \mathrm{~min}$ at $110^{\circ} \mathrm{C}$. The physical attached macromolecular film was removed by multiple washing with chloroform and sonication. Then permanently bonded PGMA layer was obtained. To modify the surfaces with initiator of ATRP, one step initiator grafting reaction was conducted. The PGMA coated substrates were immersed in a cold DMF solution $(30 \mathrm{~mL})$, and triethylamine $(0.1 \mathrm{~mL})$ and 2-bromo-2-methylpropionyl bromide $(0.1 \mathrm{~mL})$ were added drop by drop. Then the reaction was allowed to react for one night with violently stirring. Lastly, the initiator deposited substrates were used to have the polymerization just as mentioned in method 1 .

\section{Synthesis of free PMPC polymer}

PMPC was polymerized via free radical polymerization by modifying a reported literature ${ }^{31}$. In a round bottom flask, MPC (1 g, $3.4 \mathrm{mmol})$, AIBN (14 mg, $0.09 \mathrm{mmol})$ were added sequentially. Then $5 \mathrm{~mL}$ methanol was added and stirred violently under nitrogen gas. The polymerization was allowed to proceed for $8 \mathrm{~h}$ at $60{ }^{\circ} \mathrm{C}$. After that, the reaction was stopped by cooling down, and exposure to air. Diethyl ether was used to precipitate the polymer. After filtering, the precipitant was dissolved in a small amount of water and freeze dried for $24 \mathrm{~h}$.

\subsubsection{Characterization}

Static Contact Angle measurements were conducted on an optical contact angle device equipped with an electronic syringe unit (OCA15, Dataphysics, Germany). The pictures of the droplet were captured by a charge-coupled device (CCD) video camera, and the drop contour was fitted by the Young-Laplace method. At least three droplets were analyzed to determine the contact angle of specimens in each step, and the results were shown in Table 7.1 . 
Table 7.1. Contact angle results of the substrates for each step to obtain Silane-PMPC and PGMA-PMPC

\begin{tabular}{ccccccc} 
& \multicolumn{3}{c}{ Silane-PMPC $\left( \pm 2^{\circ}\right)$} & & \multicolumn{3}{c}{ PGMA-PMPC $\left( \pm 2^{\circ}\right)$} \\
after pirahna & APTES & initiator & brush & PGMA & initiator & brush \\
$14.7^{\circ}$ & $46.8^{\circ}$ & $72.5^{\circ}$ & $7.1^{\circ}$ & $54^{\circ}$ & $54.4^{\circ}$ & $6.2^{\circ}$
\end{tabular}

FTIR measurements were performed on a Bruker Vertex $70 \mathrm{v}$ spectrometer to get the specific group signals of tethered polymer brushes. Before the measurements of the substrates with brushes, a bare silicon substrate was first scanned and used as a background to be subtracted from the brush-spectra. The PMPC brush spectrum (Appendix 7.1) showed characteristic infrared absorption in $1680 \mathrm{~cm}^{-1}(\mathrm{C}=\mathrm{O}), 1260 \mathrm{~cm}^{-1}\left(\mathrm{POCH}_{2}\right), 985 \mathrm{~cm}^{-1}$ $\left(\mathrm{N}\left(\mathrm{CH}_{3}\right)_{3}\right)^{32}$. All the absorption peaks indicated that the PMPC brushes were successfully grafted from the substrates.

X-ray Photoelectron Spectroscopy (XPS) was used to determine the chemical composition of the substrates for each step modification. The measurements were carried out on a Quantera Scanning XPS Microphrobe from physical electronics with a monochromatic Al $\mathrm{K} \alpha \mathrm{X}$-ray source (1486.6 eV photons). All binding energies were referenced to the $\mathrm{C}_{1 \mathrm{~s}}$ hydrocarbon peak at $284.8 \mathrm{eV}$. The spectra showed exactly the corresponding element peak after the reaction, which gave an evidence of the successful chemical modification in each step.

Appendix 7.2 shows the XPS spectra of the surfaces after each modification step. After the first step, for the grafted PGMA layer (light gray curve), $\mathrm{C}_{1 \mathrm{~s}}(284.7 \mathrm{eV})$ and $\mathrm{O}_{1 \mathrm{~s}}(532.9 \mathrm{eV})$ peaks dominate the spectrum. Also, a $\mathrm{Si}_{2 p}$ peak is visible at $99.6 \mathrm{eV}$, which arises from the $\mathrm{SiO}_{2}$ surface underneath the thin PGMA film. The reaction of epoxy groups on the silicon substrates has been revealed from the appearance of the $\mathrm{C}-\mathrm{OH}$ peak at $285.89 \mathrm{eV}$ (Appendix 7.3). Meanwhile, still with large amounts of epoxy groups do not have reaction, which is shown at $286.84 \mathrm{eV}$. After the second step, when the initiator molecules have been grafted to the surface (dark grey curve), a characteristic $\mathrm{Br}_{3 \mathrm{~d}}$ peak appears at $70.5 \mathrm{eV}$ (see also inset), while the $\mathrm{C}_{1 \mathrm{~s}}, \mathrm{O}_{1 \mathrm{~s}}$ and $\mathrm{Si}_{2 \mathrm{p}}$ peaks are still present. After the third step, when the PMPC brushes are grafted from the surface (black curve), the $\mathrm{Br}_{3 \mathrm{~d}}$ and $\mathrm{Si}_{2 \mathrm{p}}$ peaks vanished and new peaks can be observed. The new peak at $402.5 \mathrm{eV}$ is assigned to $\mathrm{N}_{1 \mathrm{~s}}$ arising from the positively charged $\mathrm{N}^{+}$in the polymer's side chain. The peak at $133.3 \mathrm{eV}$ is assigned to $\mathrm{P}_{2 \mathrm{p}}$ and can be attributed to the $\mathrm{P}^{-}$in PMPC. The elements fraction in each step modification is shown in Table 7.2. 
Table 7.2. Elements fraction of the surface after each modification step of the PGMAmacro-initiator method (a) PGMA layer and (b) PGMA+INI (initiator grafted PGMA layer) (c) PGMA-PMPC brush. The calculated elements fraction of PMPC is shown in bracket without counting hydrogen.

\begin{tabular}{lcccccc}
$\begin{array}{l}\text { Element } \\
\text { Sample }\end{array}$ & $\begin{array}{c}\mathbf{C} \\
\text { [at \%] }\end{array}$ & $\begin{array}{c}\mathbf{N} \\
\text { [at \%] }\end{array}$ & $\begin{array}{c}\text { O } \\
\text { [at \%] }\end{array}$ & $\begin{array}{c}\text { Si } \\
\text { [at \%] }\end{array}$ & $\begin{array}{c}\text { P } \\
\text { [at \%] }\end{array}$ & $\begin{array}{c}\text { Br } \\
\text { [at \%] }\end{array}$ \\
\hline PGMA & 65.43 & & 30.03 & 4.54 & & \\
PGMA + INI & 64.62 & & 32.67 & 2.49 & & 0.22 \\
PGMA + PMPC & 57.55 & 5.56 & 32.31 & & 4.59 & \\
& $(48)$ & $(5)$ & $(35)$ & & $(15)$
\end{tabular}

${ }^{1} \mathrm{H}$ NMR was conducted on a Bruker Avance $400 \mathrm{MHz}$ instrument, in $\mathrm{D}_{2} \mathrm{O}$. For getting the molecular weight of ATRP-PMPC, the synthesized PMPC polymer by using sacrificial propargyl 2-bromoisobutyrate initiator was dissolved in $\mathrm{D}_{2} \mathrm{O}$ at a concentration of 10 $\mathrm{mg} / \mathrm{mL}$. The repeat unit number of PMPC was estimated by using the obtained spectra (Appendix 7.4). The stability of bulk PMPC in NaF was tested by comparing the spectra of PMPC $(10 \mathrm{mg} / \mathrm{mL})$ and $0.15 \mathrm{M} \mathrm{NaF}$ mixture in $\mathrm{D}_{2} \mathrm{O}$ at various mixing time. After 2 weeks, the mixture was analyzed again by capturing another ${ }^{1} \mathrm{H}$ NMR spectrum, likewise after 4 weeks. All the spectra are shown in Appendix 7.5.

The thickness of PGMA thin layer was measured by using a Variable Angle Spectroscopic Ellipsometer (VASE, J. A. Woollam). Measurements were performed as a function of photon energy (0.8-4.0 eV, corresponding to a wavelength range of 276-1550 nm) with a step size of $0.1 \mathrm{eV}$. The experiments were performed at room temperature $\left(25^{\circ} \mathrm{C}\right)$ in air. Spectra on samples were performed at three different angles of incidence $\left(65,70\right.$ and $\left.75^{\circ}\right)$. The ellipsometry data is fitted to the traditional Cauchy model, in which thickness $\mathrm{d}$, and Cauchy parameters A and B were varied; the Cauchy parameter C was set to 0 .

AFM imaging of the brush height was carried out on a Multimode 8 AFM, with a NanoScope V controller, and a JV vertical engage scanner using tapping mode. The aluminum coated cantilevers were purchased from Olympus. The resonance frequency of the cantilevers was around $70 \mathrm{kHz}$, with the force constant of $2 \mathrm{~N} / \mathrm{m}$. All the samples were gently scratched on several positions by tweezers to expose the silicon surface underneath, which allowed for measuring the height. The brush specimens that had been separately immersed in water, $0.15 \mathrm{M} \mathrm{NaF}, \mathrm{NaCl}$ and $\mathrm{NaI}$ aqueous solution for various times, were rinsed with water and ethanol sequentially, and dried with nitrogen before AFM height measurement. Generally, three different positions of the surface were measured and the average height was taken. 


\subsubsection{Stability test}

To get the degrafting information of PMPC brush synthesized by two methods, $0.15 \mathrm{M} \mathrm{NaF}$, $\mathrm{NaCl}, \mathrm{NaI}$ aqueous solution were made separately. After immersing the sample in MiliQ water or one of the salt solution for a certain time, the sample was rinsed with water and ethanol sequentially, then dried with nitrogen gas. Next, the height of the brush was measured. Afterwards, the samples were re-immersed in fresh solutions (to prevent changes in the $\mathrm{pH}$ by potential oxidation of the ions).

\subsection{Results and Discussion}

Scheme 7.1 shows the fabrication method described here to prepare PMPC brushes by surface initiated atom transfer polymerization ${ }^{30}$ using a macro-initiator. ${ }^{33}$ We have chosen PGMA as macro-initiator because of its relatively high hydrophobicity (contact angle is $54^{\circ}$ ) to prevent water from reaching the hydrolysis-sensitive bonds. In the first step, the PGMA is coupled to a piranha-activated silicon substrate using dip-coating ( $24 \mathrm{~h}$ aging time) followed by annealing for $30 \mathrm{~min}$ at $110{ }^{\circ} \mathrm{C}$. Via a ring-opening reaction of the epoxy groups with the hydroxyl groups on the $\mathrm{SiO}_{2}$, the PGMA is covalently bonded to the surface resulting in a $4 \pm 1 \mathrm{~nm}$ thick PGMA film as measured by ellipsometry after sonication in chloroform for $1 \mathrm{~min}$ such that physisorbed PGMA is removed. In the second step, the BIBB is coupled in a DMF solution $(30 \mathrm{~mL}$ with $0.1 \mathrm{~mL}$ triethylamine and $0.1 \mathrm{~mL}$ $\mathrm{BIBB})$ to the hydroxyl groups that were formed during the ring opening reaction. In contrast to the BAA or BPA used in Luzonov's papers, ${ }^{28-29}$ for which the hydroxyl group of these molecules can open the epoxy ring, our BIBB will not react with the epoxy groups on the PGMA. We note that BIBB can react with leftover hydroxyl groups on the silicon surface. However, most of these hydroxyl groups will have oxidized or reacted during annealing. In the final preparation step, the PMPC brushes are grafted from the surface using a well-established recipe. ${ }^{34}$ More details on the sample preparation and characterization can be found in the Materials and Methods. The brushes so obtained are smooth and homogeneous as shown in the AFM images in Appendix 7.6. The successful completion of the surface modification steps are tested by contact angle ${ }^{35}$ (Table 7.1) and $\mathrm{X}$-ray photoelectron spectroscopy (XPS) measurements (Appendix 7.2).

The PMPC growth kinetics for our macro-initiator method and for the commonly employed method of growing PMPC brushes using (3-aminopropyl)triethoxysilane (APTES) molecules as surface anchors are shown in Appendix 7.7. The rate of increase in dry brush height for PGMA-PMPC is lower than for silane-PMPC. The reason for this is that the initiator surface-density for the PGMA initiator method is lower compared to the initiator density for the silane-based method, which is evidenced by the difference in brush grafting densities: the PGMA-PMPC grafting density is 0.18 chains $/ \mathrm{nm}^{2}$, while our silane-PMPC 
grafting density is 0.66 chains $/ \mathrm{nm}^{2}$. We calculated the grafting density using the dry height of test samples (around $11 \mathrm{~nm}$ for PGMA-PMPC and $52 \mathrm{~nm}$ for silane-PMPC) measured by AFM and the molar mass $\left(M_{n}=5 \pm 0.3 \times 10^{4} \mathrm{~g} / \mathrm{mol}\right)$ as determined by nuclear magnetic resonance ( $\left.{ }^{1} \mathrm{H} \mathrm{NMR}\right)$ measurements of polymers grown from sacrificial propargyl 2bromoisobutyrate initiator molecules free in solution during the polymerization. ${ }^{36}$ The ${ }^{1} \mathrm{H}$ NMR spectrum is given in Appendix 7.4.

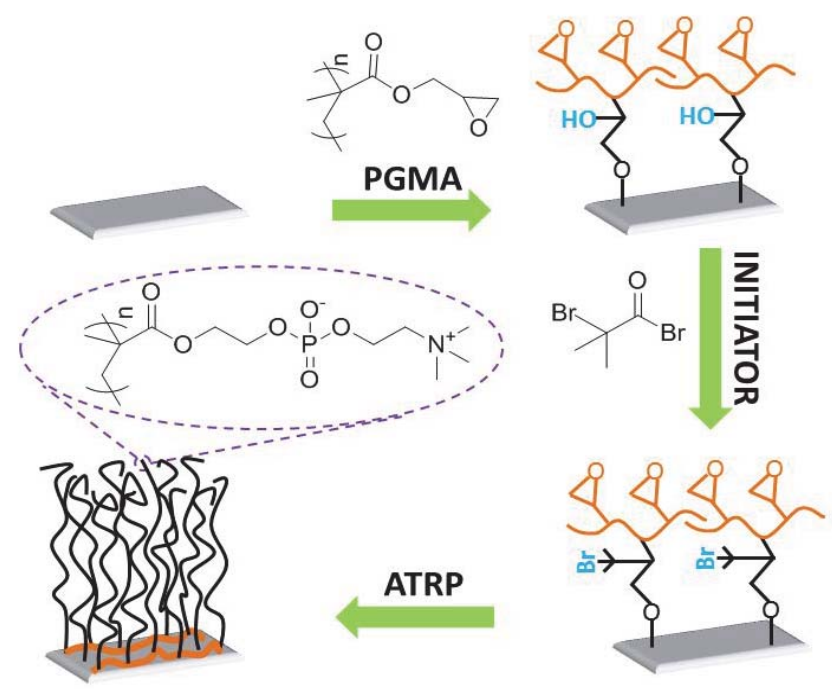

Scheme 7.1. Synthetic route for preparing PMPC brushes using the PGMA-macro-initiator method.

Before we evaluate the stability of our PGMA-PMPC brushes in aqueous solutions, we first characterize the degrafting of our reference system, which is the PMPC brush grown using trimethoxysilane-based SI-ATRP. Figure 7.1 shows the dry height of the brushes normalized by the initial dry height (typically $120 \mathrm{~nm}$ ) as a function of the immersion time of the samples in MilliQ water (upward triangles), $0.15 \mathrm{M}$ sodium fluoride (NaF, downward triangles), sodium chloride ( $\mathrm{NaCl}$, circles) and sodium iodide ( $\mathrm{NaI}$, squares). The height is measured by AFM using tapping mode. Before each height measurement, the brushes are washed with water and ethanol to remove the salt and then dried in a stream of nitrogen gas. After the measurements, the brushes are placed back into the aqueous solution. For the brush that is kept in the NaF solution, we observe the fastest decrease in dry brushheight. In the first $5 \mathrm{~h}$ almost $80 \%$ of the volume of the polymer brush detaches from the surface. After 4 days, the polymer chains making up the brushes have been totally removed from the surface. The fast degrafting can be attributed to small amounts of HF that are formed in the solution due to the slightly basic nature of the $\mathrm{F}^{-}$ions. HF is a regularly employed component of wet etchants for silicon-oxide surfaces ${ }^{37}$ via the reaction: $\mathrm{SiO}_{2}+6 \mathrm{HF} \rightleftharpoons 2 \mathrm{H}^{+}+\mathrm{SiF}_{6}{ }^{2-}+2 \mathrm{H}_{2} \mathrm{O}$. It has been employed to remove brushes for further analysis by others. ${ }^{38}$ Polymer-degradation, e.g. by hydrolysis of the ester bond in 
PMPC, is excluded as a cause for decreasing the brush height by ${ }^{1} \mathrm{H}$ NMR measurements (see Appendix 7.5), which show that PMPC polymers are still intact after 4 weeks of immersion in a $0.15 \mathrm{M} \mathrm{NaF}$ solution.

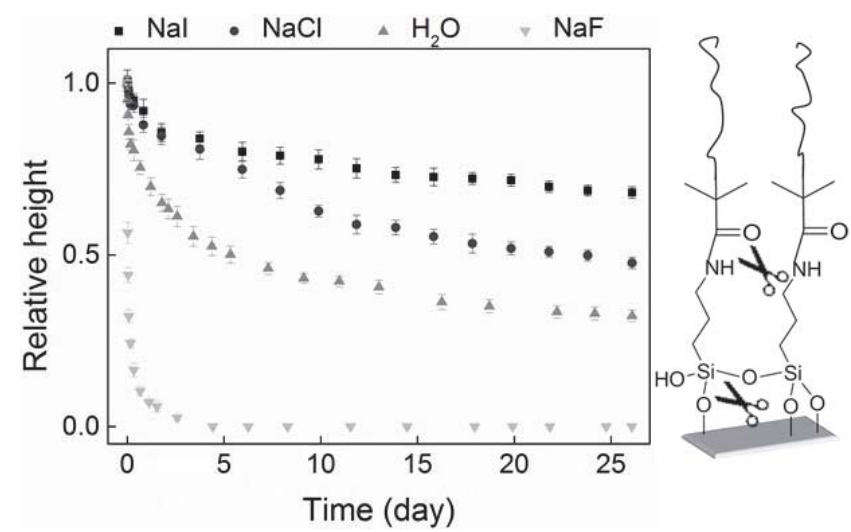

Figure 7.1. Degrafting of Silane-PMPC brushes (prepared in the same batch) in MilliQ water (upward triangles), $0.15 \mathrm{M} \mathrm{NaF}$ (downward triangles), $\mathrm{NaCl}$ (circles) and $\mathrm{NaI}$ (squares) solution. The height is normalized by the initial height (typically around $120 \mathrm{~nm}$ ).

The brushes that are kept in MilliQ water and in $0.15 \mathrm{M} \mathrm{NaCl}$ and $\mathrm{NaI}$ solutions degraft slower than the brushes kept in $\mathrm{NaF}$ solution (see Figure 7.1). After 26 days of immersion time, the measured height is still $32 \%$ of the initial height for water, $48 \%$ for the $\mathrm{NaCl}$ solution and $68 \%$ for the NaI solution. There are two possible mechanisms for degrafting in these solutions. There can be hydrolysis of the Si-O bond ${ }^{14 a}$ : $-\mathrm{Si}-\mathrm{O}-\mathrm{Si}-\longrightarrow-\mathrm{Si}-\mathrm{OH}$, or the amide bond ${ }^{40}: \mathrm{R}_{1} \mathrm{CO}-\mathrm{NHR}_{2} \rightleftharpoons \mathrm{R}_{1} \mathrm{COOH}+\mathrm{H}_{2} \mathrm{NR}_{2}$. Hydrolysis of these bonds typically occur at extreme $\mathrm{pH}$ or temperature. But, tension can shift the hydrolysis equilibrium such that hydrolysis can occur at neutral $\mathrm{pH}$ and room temperature too. ${ }^{39}$ It is difficult to predict where the polymer will be cleaved off. The activation energy for amide hydrolysis (theory $63-113 \mathrm{~kJ} / \mathrm{mol}^{40}$ and experiment $76-105 \mathrm{~kJ} / \mathrm{mol}^{41}$ ) is slightly higher than for Si-O hydrolysis (theory $71-142 \mathrm{~kJ} / \mathrm{mol}^{42}$ and experiment $67-96 \mathrm{~kJ} / \mathrm{mol}^{43}$ ). The energies for direct bond dissociation within the molecules are much larger $\left(350-450 \mathrm{~kJ} / \mathrm{mol}^{44}\right)$ and, thus, direct dissociation is less likely to occur.

Figure 7.1 also shows that the decrease in height as a function of time shows a roughly similar functional dependence for all three datasets. After an initial quick decrease, the polymer detachment evolves linearly in time at a rate of $0.47 \pm 0.1 \%$ per day. We attribute the initial quick decay to tension-enhanced hydrolysis at the anchor points, as observed by others. ${ }^{15 a}$ It is difficult to predict what the exact functional dependence of the initial decay should be, since there is a nonlinear dependence of the osmotic pressure (and thus tension) on the grafting density. ${ }^{45}$ This dependency is known for semi-dilute brushes, but is 
undetermined for all grafting densities. Moreover, to our knowledge, it is unknown how tension affects the hydrolysis equilibrium for amide and siloxane bonds.

The initial quick decay strongly depends on the specific ions in the solutions, which is a result of the difference in brush swelling and, thus, polymer stretching and tension at the anchor points. As shown in Appendix 7.8, we observe the strongest polymer stretching in pure water (swelling ratio 6), for which we also observe the fastest initial degrafting. The brush swelling slightly decreases by $26 \%$ in $\mathrm{NaCl}$ and $32 \%$ in $\mathrm{NaI}$ solutions, which is consistent with the slower degrafting observed for the brushes in these solutions. In a recent publication, it was reported that the addition of $\mathrm{NaCl}$ can enhance polymer detachment for poly(acrylic acid) (PAA) brushes, even though the polymers stretch less in salt solutions ${ }^{46}$. However, for these brushes degrafting is caused by a salt-induced ionization of the undissociated repeating units near the anchorpoints, which cannot be the mechanism for detachment of our polymers. The final linear decay of the height for longer immersion times (Figure 7.1) evolves independent of the specific ion (within the experimental error). This degrafting process can be due to hydrolysis, that is not tension-enhanced, or by the mechanical stress imposed during rinsing and drying of the samples before measurement.

As discussed earlier, we observe a specific ion effect on the brush swelling (and consequently the degrafting). Brushes swell the most and degraft the fastest in pure water. The addition of salt $(0.15 \mathrm{M} \mathrm{NaCl}$ or $\mathrm{NaI})$ reduces brush swelling and the degrafting rate. This effect is larger for $\mathrm{NaI}$ than for $\mathrm{NaCl}$. This indicates that larger $\mathrm{I}^{-}$anions can break up hydration more than the smaller $\mathrm{Cl}^{-}$ions. A 'salting out' effect by larger ions is inconsistent with the original Hofmeister series, ${ }^{47}$ which is a classification of ions according to their ability to precipitate proteins. However, we cannot expect agreement, because the charge(distribution) for PMPC is different from that of proteins, which is known to alter the Hofmeister series. ${ }^{48}$ Instead, our results can be explained by 'the law of matching water affinities, ${ }^{49}$ which states that cations and anions will form stable ion pairs when their hydration enthalpies match. This law is regularly employed to rationalize specific ion effects for polymers. ${ }^{50}$ Both the phosphocholines and $\mathrm{I}^{-}$ions have a weak interaction with water and can be considered to be chaotropes. ${ }^{51}$ Thus, their water-affinities match. Moreover, it was shown that $\mathrm{I}^{-}$ions bind more strongly to zwitterionic diphytanoyl phosphatidylcholine headgroups in a lipid bilayer than $\mathrm{Cl}^{-}$ions, ${ }^{52}$ which is consistent with our observation. Because of the interactions between $\mathrm{I}^{-}$ions and the phosphocholine groups in our brushes, the cooperative hydration and, thus, polymer stretching is reduced compared to pure water.

The results presented in Figure 7.1 are reproducible for brushes prepared in the same batch, because they have very similar grafting densities and degrees of polymerization (dry brush heights vary typically around $8 \%$ ). For brushes prepared in different batches, which, thus, have different degrees of polymerization and grafting densities, we observe different degrafting kinetics. In general, for higher grafting densities and longer polymers, the initial, 
quick degrafting is relatively more severe and more rapid. This is consistent with the picture of a tension-induced shift in the hydrolysis equilibrium.

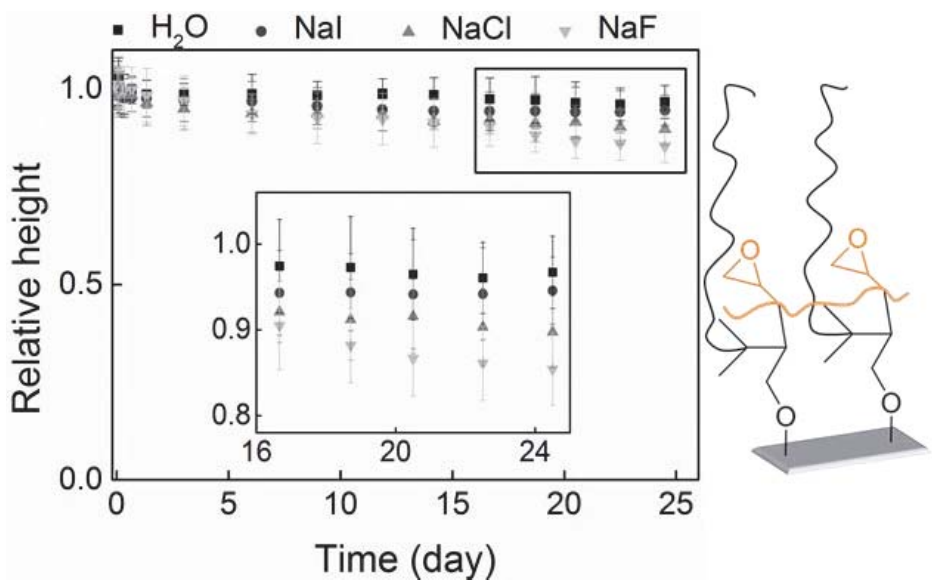

Figure 7.2. Stability of PGMA-PMPC in MilliQ water, $0.15 \mathrm{M} \mathrm{NaF}, \mathrm{NaCl}$, $\mathrm{NaI}$ solution. The inset is a magnification height over the last measurement week in which a small degrafting is observed. The height is normalized by the initial height (typically around 20 $\mathrm{nm})$

Figure 7.2 shows the normalized height change of PGMA-PMPC brushes as a function of time for immersion in water, and in $0.15 \mathrm{M} \mathrm{NaF}, \mathrm{NaCl}, \mathrm{NaI}$ solutions. In contrast to the silane-PMPC brushes, these brushes are very stable in all tested solutions. After 25 days, the dry height of the brushes has decreased by $14 \%$ in $\mathrm{NaF}$ solution, by $10 \%$ in $\mathrm{NaCl}$ solution, by $5 \%$ in $\mathrm{NaI}$ solution and by $3 \%$ in pure water. One reason for the enhanced stability can be the existence of Si-O-C surface bonds instead of Si-O-Si surface bonds. The former are less sensitive to hydrolysis. ${ }^{53}$ Moreover, it has been shown theoretically and experimentally that ester hydrolysis, which normally only occurs in acidic or basic solution, is not affected by tension. ${ }^{54}$ Thus, also the ester bonds (in initiator) are also less likely to be cleaved. Another important reason can be the hydrophobicity of the PGMA (contact angle 54 degrees), which lowers the chance of water molecules and ions reaching the hydrolysis sensitive surface bonds. In fact, when we increase the hydrophilicity of the PGMA by coupling with hydrophilic polyethylenimine (PEI) before polymerization, the brushes degraft (Appendix 7.9). Especially in NaF solution, the polymer height decreases within 1 day by $37 \%$ for PGMA-PEI-PMPC. 


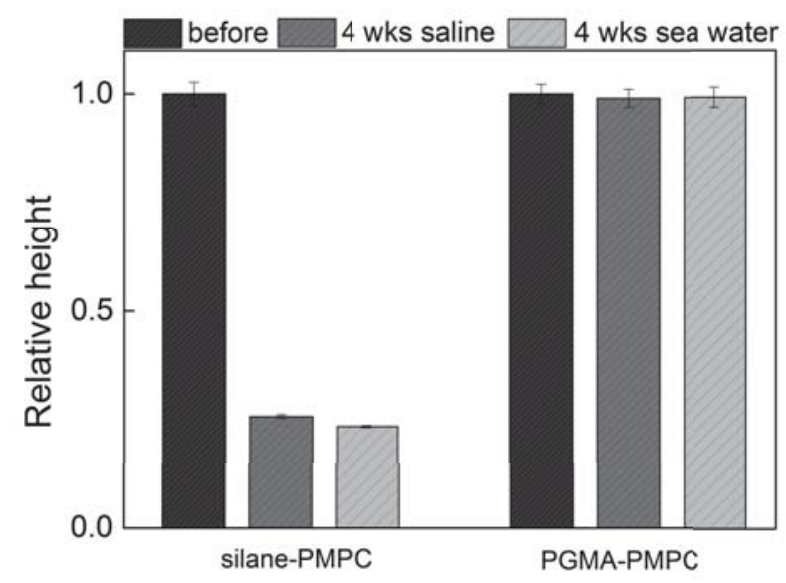

Figure 7.3. Stability of silane-PMPC and PGMA-PMPC in physiological media (saline) and sea water $(0.6 \mathrm{M} \mathrm{NaCl})$ before and after 4 weeks.

PMPC shows an excellent biocompatibility. ${ }^{55}$ Therefore, we anticipate that PMPC brushes can be applied as low-friction and low-fouling coatings in the human body, e.g. on artificial joints. Other applications could be in marine environments. Hence, we test the stability of our PGMA-PMPC brushes compared with silane-PMPC in saline solution and artificial seawater. For silane-PMPC (shown in Figure 7.3), there is a severe degrafting even in the first week, and about $80 \%$ of the brushes are cleaved from the substrate after 4 weeks. As shown in Figure 7.3, the PGMA-PMPC brushes are extremely stable over long times and there is as little as $1 \%$ polymer detachment after immersion for 4 weeks. These results indicate that the small degrafting observed for the brushes of Figure 7.2 can in part be induced by the shear stress that has been regularly applied during rinsing and drying the samples. But, more importantly, our results show that our PGMA-PMPC brushes hold great potential for application in biomedical applications and marine antifouling coatings.

\subsection{Conclusions}

In summary, we have shown that zwitterionic PMPC brushes can be successfully grafted from PGMA that is covalently bonded to a silicon surface. To enable initiation of the polymerization we couple $\mathrm{BIBB}$ initiator molecules to the hydroxyl groups within the linker between the PGMA and the $\mathrm{SiO}_{2}$ surface. Our PGMA-PMPC brushes are shown to be very stable in pure water and salt solutions. Only $1 \%$ of the polymers detach after immersion for 4 weeks in saline solution or in artificial seawater. In contrast, PMPC brushes that are grafted using the traditionally employed silane-based surface initiated ATRP method show a strong ion-specific degrafting, which can be explained by the difference in polymer stretching in the different aqueous media. 


\subsection{Appendix}

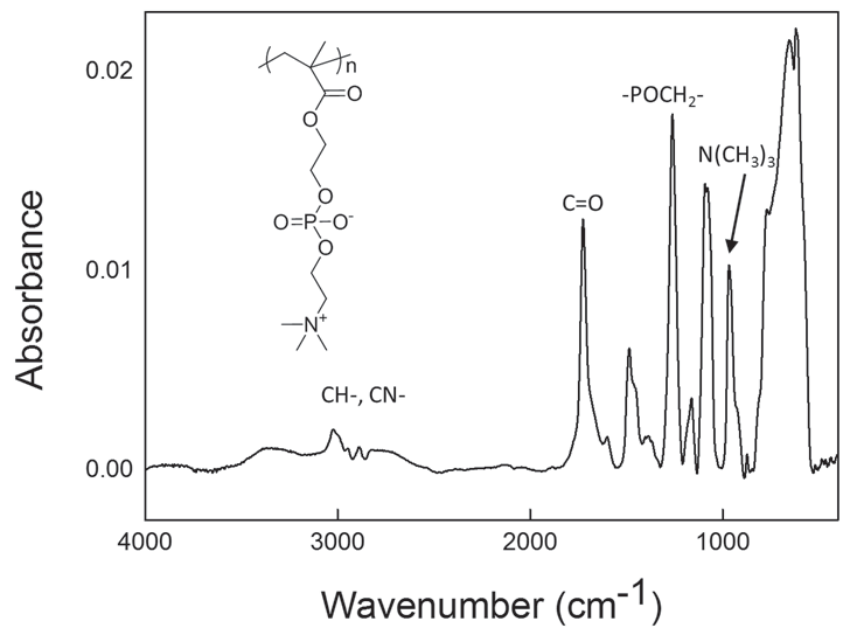

Appendix 7.1. FTIR spectrum of silane-PMPC brush on silicon substrate

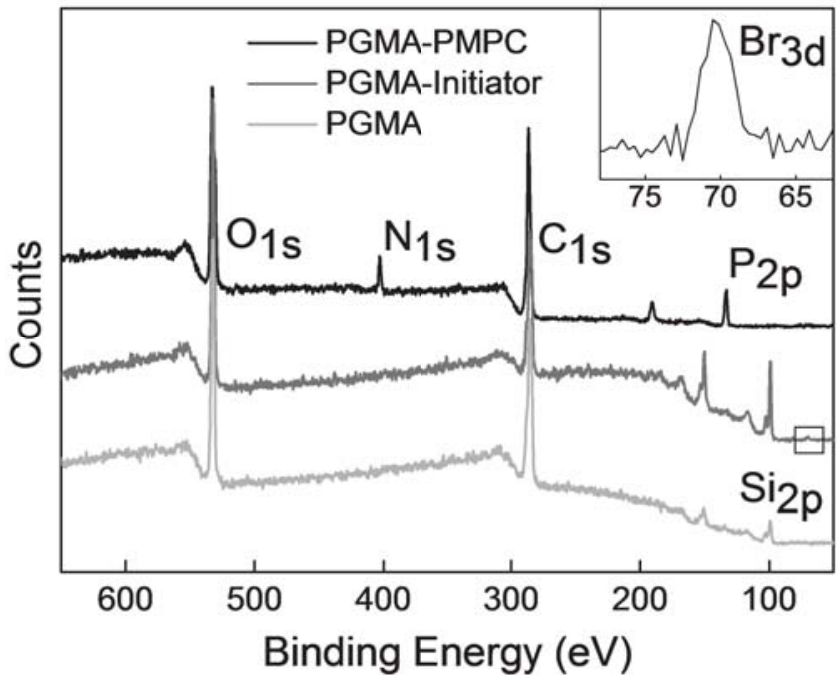

Appendix 7.2. XPS spectra of the surface after each modification step of the PGMAmacro-initiator method (a) PGMA layer and (b) initiator grafted PGMA layer (c) PGMAPMPC brush. 


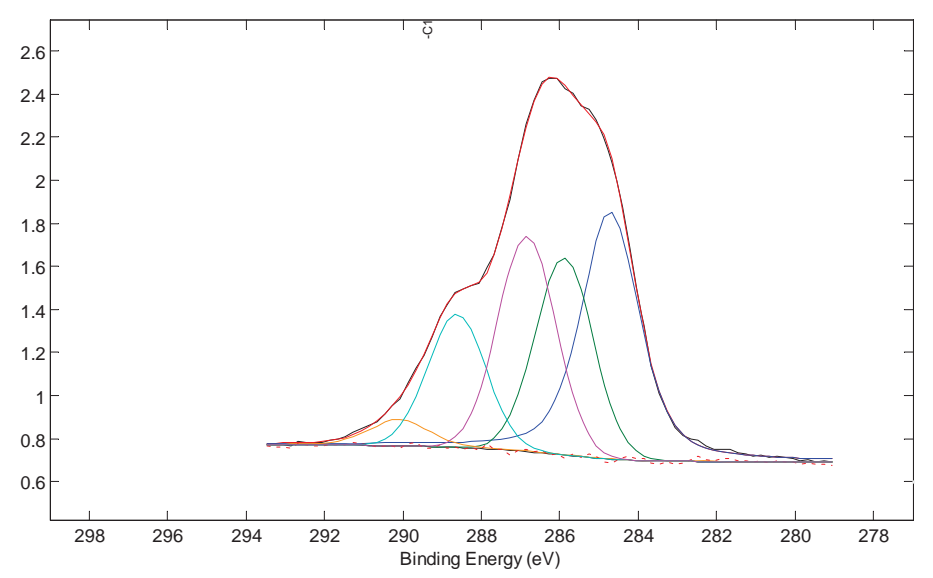

Appendix 7.3. XPS spectrum of $\mathrm{C} 1 \mathrm{~s}$ of PGMA layer, $\mathrm{C}_{1 \mathrm{~s}}$ fit after shift of the main $\mathrm{C}_{1 \mathrm{~s}}$ band to $284.71 \mathrm{eV}$ as the reference.

\begin{tabular}{crlrlrrrrl} 
Band & \multicolumn{1}{c}{ Pos PosSep B_FWHM Height \%Gauss Area \%Area } & \\
1 & 284.71 & 0.00 & 1.75 & 11478 & 54 & 26029 & 34.39 & \\
2 & 285.89 & 1.17 & 1.75 & 9143 & 100 & 16995 & 22.45 & C-OH and C-O-C \\
3 & 286.84 & 2.12 & 1.75 & 9991 & 100 & 18573 & 24.54 & EPOXY \\
4 & 288.62 & 3.91 & 1.75 & 6185 & 100 & 11497 & 15.19 & \\
5 & 290.10 & 5.39 & 1.75 & 1228 & 71 & 2596 & 3.43 &
\end{tabular}

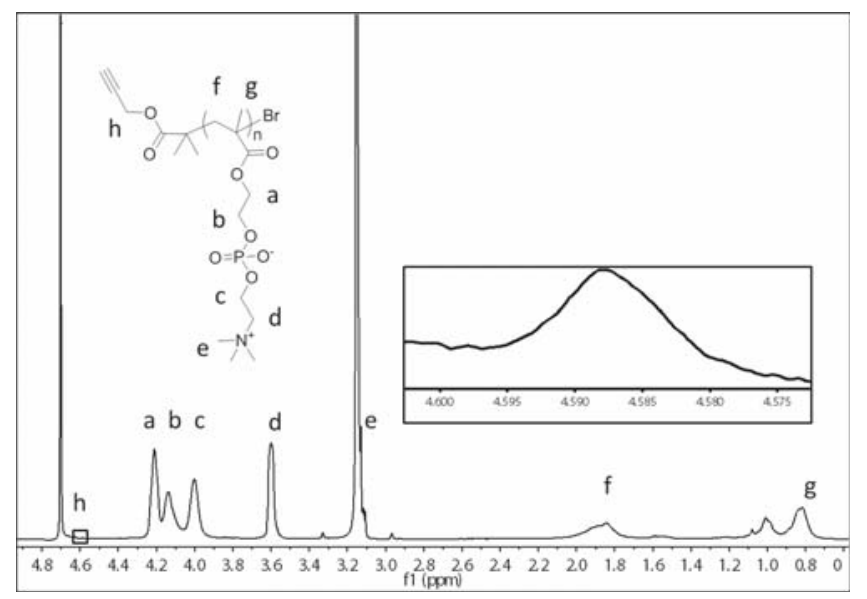

Appendix 7.4. ${ }^{1} \mathrm{H}$ NMR spectra of PMPC, which is initiated by propargyl 2bromoisobutyrate, in $\mathrm{D}_{2} \mathrm{O}$. 


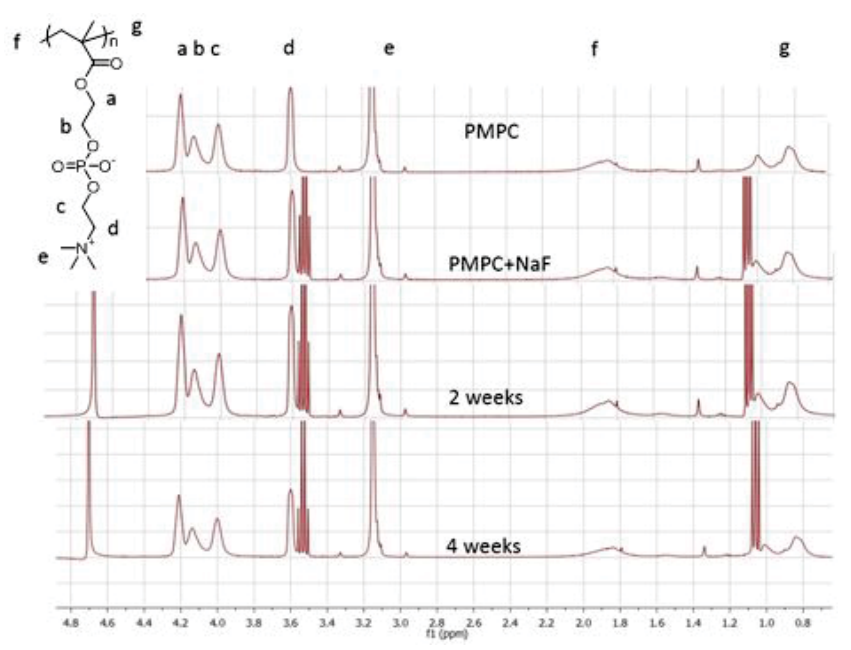

Appendix 7.5. ${ }^{1} \mathrm{H}$ NMR spectra of (a) PMPC in $\mathrm{D}_{2} \mathrm{O}$, (b) PMPC with $0.15 \mathrm{M} \mathrm{NaF}$ in $\mathrm{D}_{2} \mathrm{O}$, (c) the mixture standing for 2 weeks, (d) for 4 weeks. There is no shift on the corresponding proton signal, which indicates there is on degradation of ester bond in PMPC.

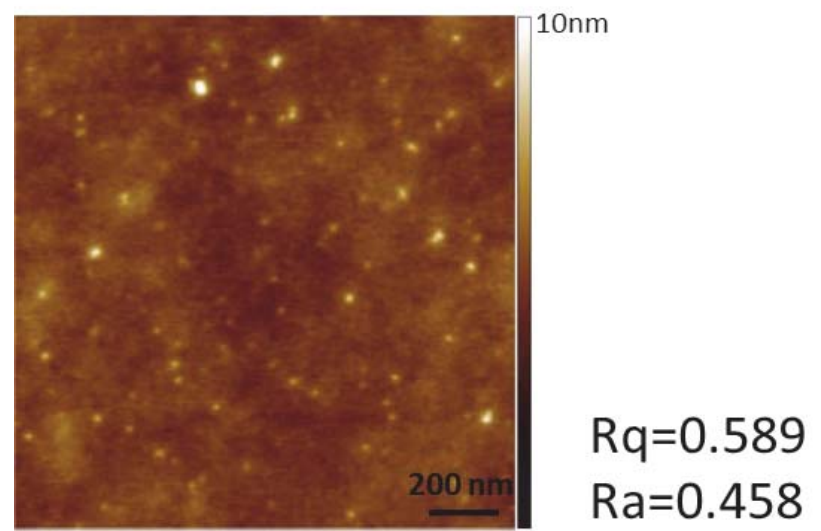

Appendix 7.6. Surface morphology of the PGMA-PMPC brushes, and the corresponding roughness. The scale bar is $200 \mathrm{~nm}$ in dimension. 


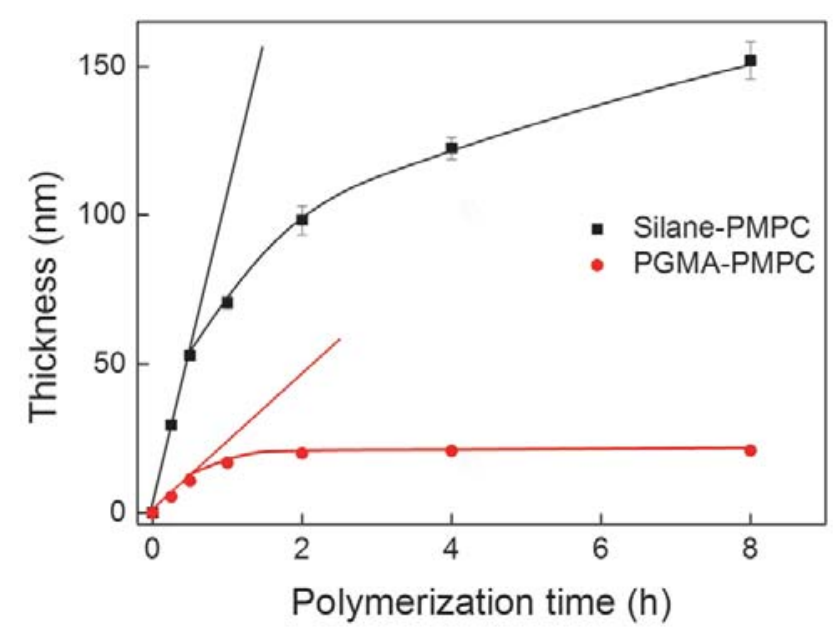

Appendix 7.7. Evolution of PMPC brush dry thickness obtained from two different methods: Silane-PMPC and PGMA-PMPC

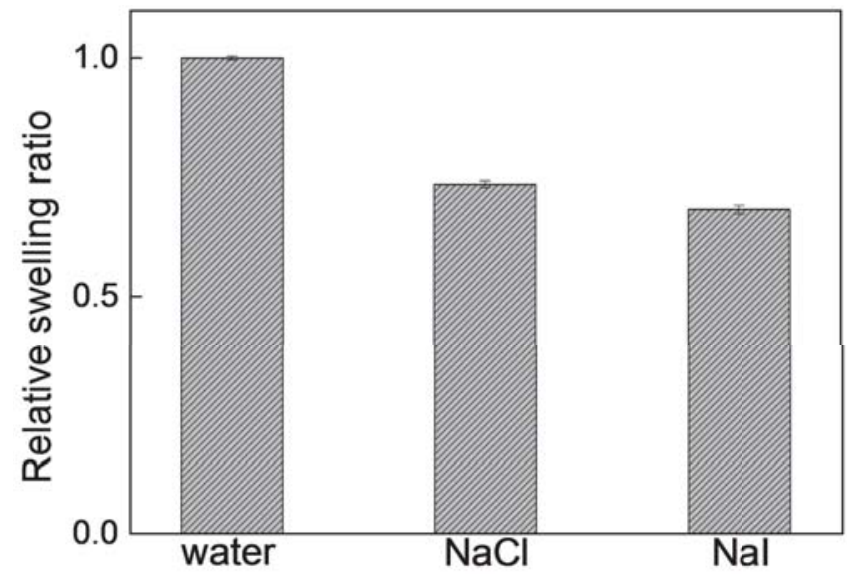

Appendix 7.8. The relative swelling ratio of PGMA-PMPC in water, $0.15 \mathrm{M} \mathrm{NaCl}$, and NaI solution separately. 


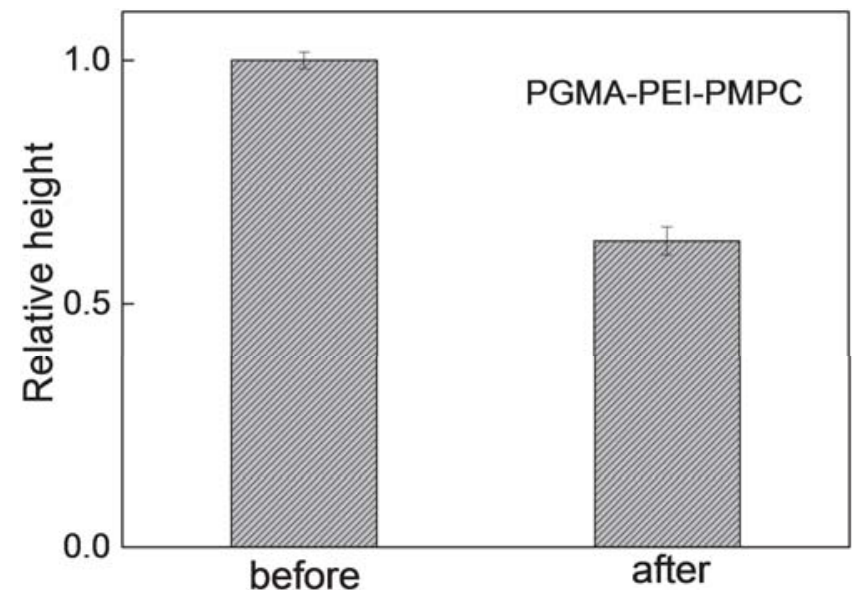

Appendix 7.9. The relative height change of PGMA-PEI-PMPC before and after immersing in $0.15 \mathrm{M} \mathrm{NaF}$ for $24 \mathrm{~h}$. 


\subsection{Reference}

[1] a) Z. H. Nie and E. Kumacheva, Nature Materials 2008, 7, 277-290; b) V. Kochkodan and N. Hilal, Desalination 2015, 356, 187-207.

[2] a) S. T. Milner, Science 1991, 251, 905-914; b) D. M. Jones, J. R. Smith, W. T. S. Huck and C. Alexander, Advanced Materials 2002, 14, 1130-1134; c) S. Minko, S. Patil, V. Datsyuk, F. Simon, K. J. Eichhorn, M. Motornov, D. Usov, I. Tokarev and M. Stamm, Langmuir 2002, 18, 289-296; d) S. Edmondson, V. L. Osborne and W. T. S. Huck, Chemical Society Reviews 2004, 33, 14-22; e) T. Chen, R. Ferris, J. M. Zhang, R. Ducker and S. Zauscher, Progress in Polymer Science 2010, 35, 94-112; f) M. A. C. Stuart, W. T. S. Huck, J. Genzer, M. Muller, C. Ober, M. Stamm, G. B. Sukhorukov, I. Szleifer, V. V. Tsukruk, M. Urban, F. Winnik, S. Zauscher, I. Luzinov and S. Minko, Nature Materials 2010, 9, 101-113.

[3] a) O. Azzaroni, A. A. Brown and W. T. Huck, Advanced Materials 2007, 19, 151-154; b) M. Kobayashi, Y. Terayama, H. Yamaguchi, M. Terada, D. Murakami, K. Ishihara and A. Takahara, Langmuir 2012, 28, 7212-7222.

[4] a) S. H. Ma, D. A. Wang, Y. M. Liang, B. Q. Sun, S. N. Gorb and F. Zhou, Small 2015, 11, 1131-1137; b) C. Rodriguez-Emmenegger, C. M. Preuss, B. Yameen, O. Pop-Georgievski, M. Bachmann, J. O. Mueller, M. Bruns, A. S. Goldmann, M. Bastmeyer and C. Barner-Kowollik, Advanced Materials 2013, 25, 6123-6127; c) Y. L. Yu, B. D. Kieviet, E. Kutnyanszky, G. J. Vancso and S. de Beer, Acs Macro Letters 2015, 4, 75-79.

[5] a) N. Nordgren and M. W. Rutland, Nano Letters 2009, 9, 2984-2990; b) M. K. Vyas, K. Schneider, B. Nandan and M. Stamm, Soft Matter 2008, 4, 1024-1032; c) Q. B. Wei, M. R. Cai, F. Zhou and W. M. Liu, Macromolecules 2013, 46, 9368-9379; d) Y. Wu, Q. B. Wei, M. R. Cai and F. Zhou, Advanced Materials Interfaces 2015, 2.

[6] a) I. Tokareva, S. Minko, J. H. Fendler and E. Hutter, Journal of the American Chemical Society 2004, 126, 15950-15951; b) H. M. Wang, L. X. Mu, L. L. Jin, G. W. She, H. T. Xu and W. S. Shi, Rsc Advances 2014, 4, 60086-60091.

[7] a) Y. Q. Shen, Y. H. Zhan, J. B. Tang, P. S. Xu, P. A. Johnson, M. Radosz, E. A. Van Kirk and W. J. Murdoch, Aiche Journal 2008, 54, 2979-2989; b) M. Motornov, T. K. Tam, M. Pita, I. Tokarev, E. Katz and S. Minko, Nanotechnology 2009, 20, 434006.

[8] a) M. Chen, W. H. Briscoe, S. P. Armes and J. Klein, science 2009, 323, 1698-1701; b) Z. Y. Zhang, A. J. Morse, S. P. Armes, A. L. Lewis, M. Geoghegan and G. J. Leggett, Langmuir 2013, 29, 10684-10692; c) M. Chen, W. H. Briscoe, S. P. Armes, H. Cohen and J. Klein, Chemphyschem 2007, 8, 1303-1306.

[9] a) R. Quintana, D. Janczewski, V. A. Vasantha, S. Jana, S. S. C. Lee, F. J. Parra-Velandia, S. F. Guo, A. Parthiban, S. L. M. Teo and G. J. Vancso, Colloids and Surfaces B-Biointerfaces 2014, 120, 118-124; b) Y. Zhang, Z. Wang, W. F. Lin, H. T. Sun, L. G. Wu and S. F. Chen, Journal of Membrane Science 2013, 446, 164-170; c) J. H. Kuang and P. B. Messersmith, Langmuir 2012, 28, 7258-7266.

[10] a) M. Ilcikova, J. Tkac and P. Kasak, Polymers 2015, 7, 2344-2370; b) T. Moro, Y. Takatori, K. Ishihara, T. Konno, Y. Takigawa, T. Matsushita, U. I. Chung, K. Nakamura and H. Kawaguchi, Nature Materials 2004, 3, 829-836.

[11] T. Moro, Y. Takatori, M. Kyomoto, K. Ishihara, M. Hashimoto, H. Ito, T. Tanaka, H. Oshima, S. Tanaka and H. Kawaguchi, Journal of Orthopaedic Research 2014, 32, 369-376.

[12] K. Kitano, Y. Inoue, R. Matsuno, M. Takai and K. Ishihara, Colloids and Surfaces B-Biointerfaces 2009, 74, 350-357.

[13] H. Furusawa, T. Sekine and T. Ozeki, Macromolecules 2016, 49, 3463-3470.

[14] a) R. Quintana, M. Gosa, D. Janczewski, E. Kutnyanszky and G. J. Vancso, Langmuir 2013, 29, 1085910867; b) M. Kobayashi, M. Terada and A. Takahara, Faraday discussions 2012, 156, 403-412; c) J. J. Wang and J. Wei, Applied Surface Science 2016, 382, 202-216.

[15] a) H. A. Klok and J. Genzer, Acs Macro Letters 2015, 4, 636-639; b) S. Tugulu and H. A. Klok, Biomacromolecules 2008, 9, 906-912; c) K. Enomoto, S. Takahashi, T. Iwase, T. Yamashita and Y. Maekawa, Journal of Materials Chemistry 2011, 21, 9343-9349.

[16] a) S. R. Wasserman, Y. T. Tao and G. M. Whitesides, Langmuir 1989, 5, 1074-1087; b) B. Liberelle and S. Giasson, Langmuir 2007, 23, 9263-9270.

[17] a) E. D. Bain, K. Dawes, A. E. Ozcam, X. F. Hu, C. B. Gorman, J. Srogl and J. Genzer, Macromolecules 2012, 45, 3802-3815; b) D. Cimen, T. T. Kursun and T. Caykara, Journal of Polymer Science Part a-Polymer Chemistry 2014, 52, 3586-3596; c) R. R. Patil, S. Turgman-Cohen, J. Srogl, D. Kiserow and J. Genzer, Acs Macro Letters 2015, 4, 251-254; d) Y. X. Zhang, B. E. Lv, Z. Y. Lu, J. A. He, S. Zhang, H. Chen and H. W. Ma, Soft Matter 2011, 7, 11496-11500; e) B. E. Lv, Y. T. Zhou, W. L. Cha, Y. Z. Wu, J. X. Hu, L. Q. Li, L. F. Chi and H. W. Ma, Acs Applied Materials \& Interfaces 2014, 6, 8313-8319.

[18] S. S. Sheiko, S. Panyukov and M. Rubinstein, Macromolecules 2011, 44, 4520-4529.

[19] K. A. Melzak, K. Yu, D. Bo, J. N. Kizhakkedathu and J. L. Toca-Herrera, Langmuir 2015, 31, 6463-6470. 
[20] a) Y. Hoshi, Y. Y. Xu and C. K. Ober, Polymer 2013, 54, 1762-1767; b) C. J. Kang, R. M. Crockett and N. D. Spencer, Macromolecules 2014, 47, 269-275.

[21] a) Z. H. Wang and H. Zuilhof, Journal of Materials Chemistry A 2016, 4, 2408-2412; b) H. Ma, J. Hyun, P. Stiller and A. Chilkoti, Advanced Materials 2004, 16, 338-341; c) D. Paripovic and H. A. Klok, Macromolecular Chemistry and Physics 2011, 212, 950-958.

[22] R. Ogaki, O. Z. Andersen, G. V. Jensen, K. Kolind, D. C. E. Kraft, J. S. Pedersen and M. Foss, Biomacromolecules 2012, 13, 3668-3677.

[23] O. Borozenko, V. Machado, W. G. Skene and S. Giasson, Polymer Chemistry 2014, 5, 5740-5750.

[24] X. F. Sui, A. Di Luca, M. K. Gunnewiek, E. S. Kooij, C. A. van Blitterswijk, L. Moroni, M. A. Hempenius and G. J. Vancso, Australian Journal of Chemistry 2011, 64, 1259-1266.

[25] C. M. Comisar, S. E. Hunter, A. Walton and P. E. Savage, Industrial \& Engineering Chemistry Research 2008, 47, 577-584.

[26] L. Richert, F. Boulmedais, P. Lavalle, J. Mutterer, E. Ferreux, G. Decher, P. Schaaf, J. C. Voegel and C. Picart, Biomacromolecules 2004, 5, 284-294.

[27] M. Divandari, E. S. Dehghani, N. D. Spencer, S. N. Ramakrishna and E. M. Benetti, Polymer 2016, 98, 470-480.

[28] Y. Liu, V. Klep, B. Zdyrko and I. Luzinov, Langmuir 2004, 20, 6710-6718.

[29] Y. Liu, V. Klep, B. Zdyrko and I. Luzinov, Langmuir 2005, 21, 11806-11813.

[30] K. Matyjaszewski, Macromolecules 2012, 45, 4015-4039.

[31] A. L. Lewis, P. D. Hughes, L. C. Kirkwood, S. W. Leppard, R. P. Redman, L. A. Tolhurst and P. W. Stratford, Biomaterials 2000, 21, 1847-1859.

[32] a) X. Y. Sun, S. S. Yu, J. Q. Wan and K. Z. Chen, Journal of Biomedical Materials Research Part A 2013, 101, 607-612; b) K. L. Chen, S. X. Zhou and L. M. Wu, Rsc Advances 2015, 5, 104907-104914.

[33] S. Edmondson and S. P. Armes, Polymer International 2009, 58, 307-316.

[34] A. J. Morse, S. Edmondson, D. Dupin, S. P. Armes, Z. Zhang, G. J. Leggett, R. L. Thompson and A. L. Lewis, Soft Matter 2010, 6, 1571-1579.

[35] Y. L. Yu, B. D. Kieviet, F. Liu, I. Siretanu, E. Kutnyanszky, G. J. Vancso and S. de Beer, Soft Matter 2015, 11, 8508-8516.

[36] a) M. Kobayashi, M. Terada, Y. Terayama, M. Kikuchi and A. Takahara, Macromolecules 2010, 43, 8409-8415; b) W. Z. Yuan, L. L. Li and H. Zou, Rsc Advances 2015, 5, 80264-80268.

[37] J. Buhler, F. P. Steiner and H. Baltes, Journal of Micromechanics and Microengineering 1997, 7, R1R13.

[38] T. von Werne and T. E. Patten, Journal of the American Chemical Society 2001, 123, 7497-7505.

[39] S. W. Schmidt, A. Kersch, M. K. Beyer and H. Clausen-Schaumann, Physical Chemistry Chemical Physics 2011, 13, 5994-5999.

[40] a) J. P. Guthrie, Journal of the American Chemical Society 1974, 96, 3608-3615; b) Y. Xiong and C. G. Zhan, Journal of Physical Chemistry A 2006, 110, 12644-12652; c) D. Zahn, Chemical Physics Letters 2004, 383, 134-137.

[41] a) T. M. Alm, Polymer 2003, 44, 6531-6536; b) A. Meyer, N. Jones, Y. Lin and D. Kranbuehl, Macromolecules 2002, 35, 2784-2798; c) H. Slebocka-Tilk, F. Sauriol, M. Monette and R. S. Brown, Canadian Journal of Chemistry-Revue Canadienne De Chimie 2002, 80, 1343-1350.

[42] a) Y. T. Xiao and A. C. Lasaga, Geochimica Et Cosmochimica Acta 1996, 60, 2283-2295; b) Y. T. Xiao and A. C. Lasaga, Geochimica Et Cosmochimica Acta 1994, 58, 5379-5400; c) J. E. Del Bene, K. Runge and R. J. Bartlett, Computational Materials Science 2003, 27, 102-108; d) M. Cypryk and Y. Apeloig, Organometallics 2002, 21, 2165-2175.

[43] a) W. Hertl, Journal of Physical Chemistry 1968, 72, 3993-\&; b) J. D. Rimstidt and H. L. Barnes, Geochimica Et Cosmochimica Acta 1980, 44, 1683-1699; c) P. M. Dove and D. A. Crerar, Geochimica Et Cosmochimica Acta 1990, 54, 955-969.

[44] a) T. L. Cottrell, The strengths of chemical bonds, Academic Press, 1958, p; b) R. Sanderson, Chemical Bonds and Bonds Energy, Elsevier, 2012, p.

[45] P. G. Degennes, Macromolecules 1980, 13, 1069-1075.

[46] B. Lego, W. G. Skene and S. Giasson, Macromolecules 2010, 43, 4384-4393.

[47] W. Kunz, J. Henle and B. W. Ninham, Current Opinion in Colloid \& Interface Science 2004, 9, 19-37.

[48] X. Chen, T. Yang, S. Kataoka and P. S. Cremer, Journal of the American Chemical Society 2007, 129, 12272-12279.

[49] K. D. Collins, Biophysical journal 1997, 72, 65.

[50] A. Salis and B. W. Ninham, Chemical Society Reviews 2014, 43, 7358-7377.

[51] N. Vlachy, B. Jagoda-Cwiklik, R. Vacha, D. Touraud, P. Jungwirth and W. Kunz, Advances in Colloid and Interface Science 2009, 146, 42-47. 
[52] J. J. Garcia-Celma, L. Hatahet, W. Kunz and K. Fendler, Langmuir 2007, 23, 10074-10080.

[53] N. S. Bhairamadgi, S. P. Pujari, F. G. Trovela, A. Debrassi, A. A. Khamis, J. M. Alonso, A. A. Al Zahrani, T. Wennekes, H. A. Al-Turaif, C. van Rijn, Y. A. Alhamed and H. Zuilhof, Langmuir 2014, 30, 5829-5839.

[54] S. Akbulatov, Y. C. Tian, E. Kapustin and R. Boulatov, Angewandte Chemie-International Edition 2013, 52, 6992-6995.

[55] J. Z. Du, Y. Q. Tang, A. L. Lewis and S. P. Armes, Journal of the American Chemical Society 2005, 127 , 17982-17983. 



\section{Chapter 8}

\section{First Results and Outlook on: Specific Anion Effects on the Hydration and Tribological properties of Zwitterionic PMPC Brushes}

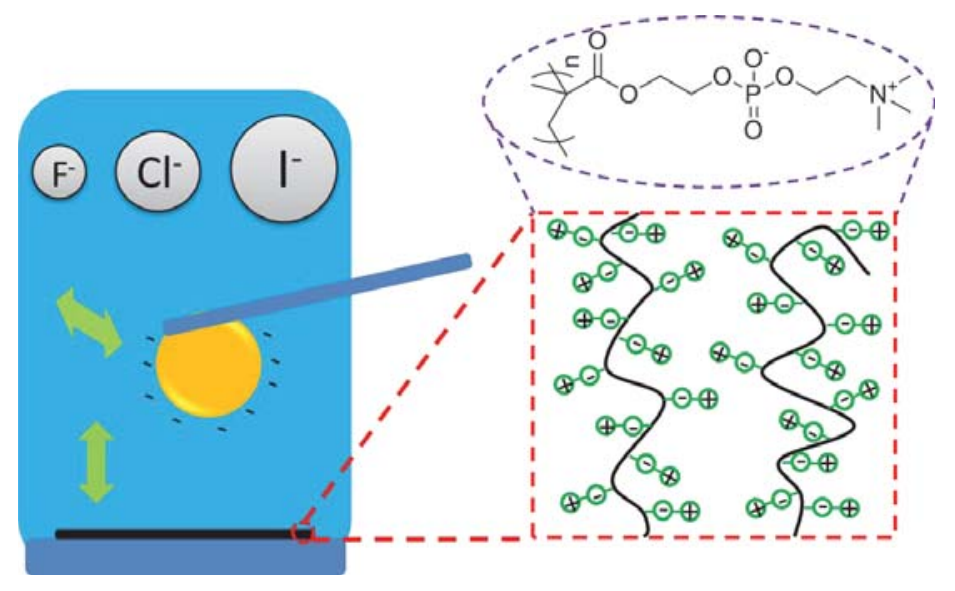

When poly(2-methacryloyloxyethyl phosphorylcholine) (PMPC) polymers are endanchored to a substrate at a high density to form so-called polymer brushes, they can act as efficient lubricants in pure water. In view of potential application of PMPC brushes on biomedical devices or in marine environments, it is important to characterize the performance of these brushes in salt solutions. In this chapter we show that the friction coefficient, which is measured upon sliding a polystyrene (PS) colloid on a PMPC brush, decreases when salt is added in the aqueous solution (1 M sodium fluoride $(\mathrm{NaF})$, sodium chloride $(\mathrm{NaCl})$, sodium iodide $(\mathrm{NaI})$, cesium chloride $(\mathrm{CsCl})$ and cesium iodide $(\mathrm{CsI})$ ). Moreover, we find that the friction coefficient decreases with increasing anionic size in the solution, while the size of the cation has no measurable effect. We observe a clear relation between the compressibility of the brush and the measured friction coefficient. In pure water, the brush absorbs large amounts of solvent and is very compressible. Under these conditions the friction coefficient is high. In salt solutions, for which we find the lowest friction coefficient for the largest anions, we find that the stiffness of the brush increases with increasing anionic size. In contrast, we find that the adhesion between the PS colloid and the PMPC brush does not relate to brush swelling and instead might arise from electrostatic interactions. We conclude this chapter with an outlook to future experiments that can clarify our observations. 


\subsection{Introduction}

In recent years, medical procedures have evolved towards minimally invasive techniques where catheters and long needles are employed to reach the region of interest in the body. For a smooth manipulation without damage of the surrounding tissue, it is of critical importance to lubricate these medical devices. Simple liquids, such as water, are easily squeezed out of the contact and, therefore, perform badly as lubricants. Polymer brushes are able to absorb low-viscosity liquids and keep them from flowing out of the contact such that the contact remains lubricated ${ }^{1}$ even when pressures are high. ${ }^{2}$

Polymer brushes are comprised of macromolecules that are attached with one end to a surface at a density that is high enough for the polymers to stretch away from the anchoring plane. ${ }^{3}$ The degree of stretching of the polymers in brushes depends on the solvent quality: the brushes collapse to form a dense film in poor solvents, while the polymers strongly stretch in good solvents. Besides being effective lubricants, such brushes can also be employed as actuators ${ }^{4}$ and sensors. ${ }^{5}$ In particular, zwitterionic polymers, which have both negative and positive charges within the same monomers, have recently been the subjects of many scientific studies. ${ }^{6}$ For example, extremely low friction has been observed between poly(2-methacryloyloxyethyl phosphorylcholine) (PMPC) brushes in pure water, ${ }^{2}$ even for pressures up to 7.5 MPa. In PMPC, the repeat unit is composed of the phosphorylcholine group, which makes them very hydrophilic. ${ }^{7}$ Moreover, these polymers are biocompatible. ${ }^{8}$ This, combined with the excellent lubricity, ${ }^{2,9}$ but also protein adsorption resistance ${ }^{10}$ in water, makes them ideal candidates for application in human body, e.g. in replacement joints ${ }^{11}$ or on the surface of needles and catheters. Since the composition of body fluids deviates from pure water, it is important to characterize how electrolytes affect the physicochemical properties of zwitterionic polymers. Due to their specific cation-anion composition, there are various phenomena that can occur depending on types of salts in their aqueous solution, ${ }^{6 \mathrm{~d}, 12}$ and ion-specific effects are anticipated.

In the 1880 's, Hofmeister and co-workers ${ }^{13}$ found that there are ion-specific effects on the precipitation of proteins from blood and egg whites, which is still extensively studied. ${ }^{14}$ Within the Hofmeister series, anions are categorized as $\mathrm{CO}_{3}{ }^{2-}>\mathrm{SO}_{4}{ }^{2-}>\mathrm{H}_{2} \mathrm{PO}_{4}^{-}>\mathrm{F}^{-}>\mathrm{Cl}^{-}>$ $\mathrm{Br}^{-} \approx \mathrm{NO}_{3}^{-}>\mathrm{I}^{-}>\mathrm{ClO}_{4}^{-}>\mathrm{SCN}^{-}$. In light of the strength of ionic hydration, the ions on the left can be classified as kosmotropes and the ions on the right as chaotropes. ${ }^{15}$ The strongly hydrated kosmotropes, tend to immobilize water molecules resulting in precipitation of the solutes. Whereas the weakly hydrated chaotropes increase water mobility and tend to increase solubility of solutes in solution. Due to these specific ion effects, various salts can be applied to control the solubility ${ }^{14 a, 16}$ and friction ${ }^{12 h, 17}$ of polymers in aqueous solutions.

The conformation of zwitterionic polymers in salt solutions depends on the segmental dipole orientation, ${ }^{18}$ on the charge density of the cationic and anionic groups ${ }^{19}$ and on the distance between the charged groups. ${ }^{20}$ For example, Kobayashi et al. ${ }^{17}$ report that poly[2- 
(methacryloyloxy)ethyl]-dimethyl(3-sulfopropyl) ammonium hydroxide (PSBMA) strongly swells while increasing the salt concentration. The same trend has also been found by Liu et $a l{ }^{20 \mathrm{~b}}$ using quartz crystal microbalance (QCM) measurements to investigate the anion specificity on poly(sulfobetaine methacrylamide) (PSBMAm) brushes. For another type of zwitterionic polymer ${ }^{12 \mathrm{~h}}$ poly (3-(1-(4-vinylbenzyl)-1H-imidazol-3-ium-3-yl)propane-1sulfonate) (PVBIPS), it has been found that the specific ions in the solution affect the hydration and friction between brushes composed of these polymers, which can be tuned reversibly by changing the salts concentration.

For PMPC it is still under debate if and how different salts affects the conformation of the polymers. ${ }^{12 e, 17}$ Through different characterization techniques, different results are obtained. For example, Mahon et al. ${ }^{12 \mathrm{e}}$ uses size exclusion chromatography to investigate the effect of the type of salt and the concentration on the hydrodynamic radii of PMPC polymers. Their results show the presence of different cation types has little effect on the radius of gyration of the polymers, but the presence of different types of anions does decrease the hydrodynamic volume. Moreover, they observe that monoatomic anions have a stronger effect than polyatomic ones. However, when dynamic light scattering is used to analyze the hydrodynamic radius of free PMPC and PMPC brushes immobilized on silica nanoparticles by Matsuda et al., ${ }^{21}$ no effect of the salt concentration could be detected. Kobayashi et al. have reported similar results. ${ }^{17}$ using atomic force microscopy (AFM) imaging measurements. Recently, Zhang et al. ${ }^{12 \mathrm{i}}$ employed ellipsometry measurements to characterize the swelling ratio of PMPC brushes in various salts solutions. Their results show that the height of the brushes slightly increases upon increasing the ionic strength. This shows that one characterization technique is not sufficient to study ion-specific effects on the swelling of PMPC.

In this chapter, we show that the swelling of PMPC brushes is smaller in salt solutions compared to pure water. Moreover, we find that the decrease is stronger for larger anion sizes, while there is no effect of the cation size. Interestingly, we observe that this effect can be hidden depending on the measurement technique employed to probe the swelling. Besides effects on the brush swelling, we also studied the friction and adhesion between PMPC brushes and polystyrene (PS) colloids. The friction between PMPC brushes and PS directly correlates with the brush swelling, while the adherence between them seems to depend on electrostatic interactions. 


\subsection{Materials and Methods}

\subsubsection{Materials}

Copper (I) bromide ( $\mathrm{CuBr}$, Aldrich, 98\%) was purified twice by stirring in acetic acid and filtered. After washing with ethanol, the light gray powder was dried in a vacuum oven at room temperature overnight. Poly(glycidyl methacrylate) (PGMA, $M_{n}=1 \times 10^{4}$ ), 2methacryloyloxyethyl phosphorylcholine (MPC, 97\%), 2,2'-bipyridyl ( $\geq$ 99\%), triethylamine (TEA, $\geq 99 \%$ ), $\alpha$-bromoisobutyryl bromide (BiBB, 98\%), copper (II) bromide $\left(\mathrm{CuBr}_{2}, \geq 99 \%\right.$ ), sodium fluoride ( $\mathrm{NaF}, \geq 99 \%$ ), sodium chloride $(\mathrm{NaCl}, \geq 99 \%)$, sodium iodide ( $\mathrm{NaI}, \geq 99.5 \%$ ), cesium chloride ( $\mathrm{CsCl}, \geq 98 \%$ ), cesium iodide (CsI, 99.9\%), $\mathrm{D}_{2} \mathrm{O}$ (99.9 atom\% D), methyl ethyl ketone (MEK) were purchased from Sigma-Aldrich, and used as received without any purification. Methanol (absolute), sulfuric acid (95-97\%), dimethylformamide (DMF), chloroform (AR), 2-proponal (AR) and toluene (AR) were purchased from Biosolve. Ethanol, hydrogen peroxide $\left(\mathrm{H}_{2} \mathrm{O}_{2}\right)$ was purchased from Merck. MilliQ water was made from a MilliQ Advantage A 10 purification system (Millipore, Billerica, Ma, USA).

\subsubsection{Synthesis of PGMA-PMPC brush}

First, the PGMA thin film was attached to Piranha cleaned substrates using dip coating in a concentration of $0.1 \%$ of PGMA in MEK solution, and dried in nitrogen atmosphere. After $48 \mathrm{~h}$ aging time, the samples were annealed for $30 \mathrm{~min}$ at $110{ }^{\circ} \mathrm{C}$ in the oven. The nonchemically bonded macromolecules were removed by washing with chloroform and sonication for 2 minutes. By a one-step reaction with $\mathrm{BiBB}$, the initiator was grafted on the substrates. Lastly, surface-initiated atom transfer radical polymerization (SI-ATRP) was conducted to graft PMPC brushes from the surfaces with a thickness of $20 \pm 2 \mathrm{~nm}$. More details on the procedure can be found in Chapter 7 .

\subsubsection{Characterization}

Static Contact Angle measurements were conducted on an optical contact angle device equipped with an electronic syringe unit (OCA15, Dataphysics, Germany). The pictures of the droplet were captured by a charge-coupled device (CCD) video camera, and the drop contour was fitted by the Young-Laplace method. At least three droplets were analyzed to determine the contact angle of specimens after each step, and the results were shown in Chapter 7.

X-ray Photoelectron Spectroscopy (XPS) was used to determine the chemical composition of the substrates for each step modification. The measurements were carried out on a Quantera scanning XPS microprobe from Physical Electronics with a monochromatic Al $\mathrm{K} \alpha \mathrm{X}$-ray source (1486.6 eV photons). All binding energies were referenced to the $\mathrm{C}_{1 \mathrm{~s}}$ hydrocarbon peak at $284.8 \mathrm{eV}$. The spectra showed exactly the corresponding element peak 
after the reaction, which gave an evidence of the successful chemical modification in each step, shown in Chapter 7.

AFM experiments were carried out on a Multimode 8 AFM (Bruker), with a NanoScope V controller, and a JV vertical engage scanner using tapping mode. The dry thickness of the PMPC brushes was measured using aluminum coated cantilevers, which were purchased from Olympus, with the resonance frequency around $70 \mathrm{kHz}$, and the force constant of 2 $\mathrm{N} / \mathrm{m}$. All the samples were gently scratched using tweezers to expose the silicon surface underneath. This allowed for measuring the relative height between silicon and brush layer. For the swelling ratio measurement in various salts solution, a liquid cell (Bruker, San Barbara, CA) and polystyrene (PS) colloid mounted probe ( $5 \mu \mathrm{m}$ diameter, TL-CONT-50, Nanosensors, Switzerland) were used. After each measurement, the sample and cantilever were washed with water and ethanol for several times to move the adsorbed salts, then dried with nitrogen gas gently. We use two different methods to extract the data, which are shown in the following paragraphs.

\subsubsection{Swelling ratio measurement}

The swollen height of the PMPC brushes is also measured using homemade colloid probes. Polystyrene (PS) colloids were glued on the tipless cantilever using a microcontroller (see also Chapter 5). The spring constant of the PS colloid probe was determined by thermal noise analysis, which gave the value of $0.3 \pm 0.03 \mathrm{~N} / \mathrm{m}$ at room temperature in aqueous solution. The deflection sensitivity of the cantilever was measured in each solution on the bare silicon surface. The force-separation curves were obtained through approaching and retracting the PS colloid to and from the PMPC brushes. The friction was measured by sliding the cantilever through the samples in the lateral direction (with the scan angle: $90^{\circ}$ ) over a sliding size $10 \mu \mathrm{m}$ using a velocity of $10 \mu \mathrm{m} / \mathrm{s}$. The cantilever is calibrated using the noncontact method resulting in a torsional conversion factor of $S_{\theta}=3.5 \times 10^{-8} \mathrm{~N} / \mathrm{V}$. After each measurement in salts solution, the substrate was rinsed with water and ethanol sequentially for three times, and dried with nitrogen before the next AFM measurement. Generally, three to four different positions of the surfaces were measured. We use two methods to obtain the swelling height of PMPC brushes.

\section{Imaging method:}

For the imaging method, we employ AFM contact mode imaging near a scratch in the brush over a distance of $30 \mu \mathrm{m}$ with a scan rate of $15 \mu \mathrm{m} / \mathrm{s}$. We use a very small normal load (typically $1-1.5 \mathrm{nN}$ ) to minimize the deformation of the brush.

\section{Force-separation method:}

First, we set the set-point of the normal load for imaging to $2.5 \mathrm{nN}$. Under this load, the relative height between bare silicon and brush was determined to get the swollen height of PMPC brushes $\left(h_{0}\right)$. Next, the force-separation data was captured on the brush over a 
distance of $2 \mu \mathrm{m}$ using a maximum load of about $50 \mathrm{nN}$ with a ramp speed of $1 \mu \mathrm{m} / \mathrm{s}$. The $h_{0}$ imaged $2.5 \mathrm{nN}$ is employed to convert the separation to 'distance from the silicon surface' by shifting the separation observed for $2.5 \mathrm{nN}$ to $h_{0}$. After the determination of our zero, we determine the extra height $(\Delta h)$, which is the difference between $h_{0}$ and the distance at which the force just starts to deviate from zero. The maximum swelling height of brush is calculated by: $h=h_{0}+\Delta h$.

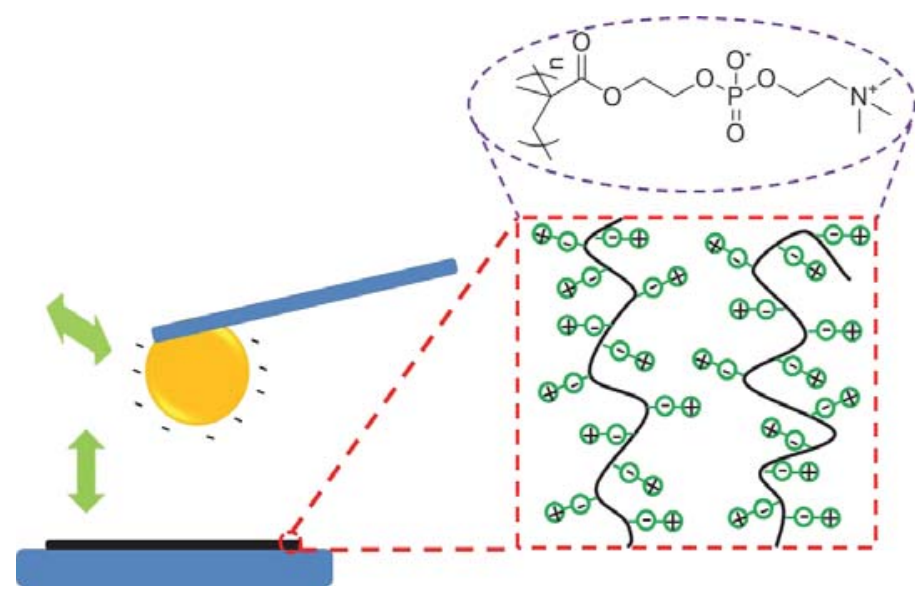

Scheme 8.1. Schematic presentation of friction and adhesion measurement on PMPC brushes using colloid probe cantilever

Scheme 8.1 depicts the friction and adhesion measurements between PMPC brushes on the surface a PS colloid on the AFM cantilever. With the movement of colloid in the normal direction, force-separation curve and adhesion force can be measured. While, in the lateral direction, friction response can be obtained.

\subsection{Results and Discussion}

Figure 8.1 shows the swelling ratio of PMPC brushes in $\mathrm{H}_{2} \mathrm{O}, 1 \mathrm{M} \mathrm{NaF}, \mathrm{NaCl}$ and $\mathrm{NaI}$ solution normalized by the swelling ratio in $\mathrm{H}_{2} \mathrm{O}$. We employed two different AFM techniques to extract the swelling ratios: (1) by imaging under a small compression $(1.5 \mathrm{nN}$, top graph) and (2) via force-separation curves (bottom graph). As can be seen from the force-separation curves captured in the various salts solutions (Figure 8.2), we find that the PMPC brushes contract compared to in pure water. When the brushes are slightly compressed by the colloid while imaging, we observe the opposite effect: the swelling ratio of the brushes increases by increasing the counterions radius.

In pure water, the swelling ratio for the PMPC brushes extracted from force distance curves (Figure 8.1, top graph) is around 10. By adding $1 \mathrm{M} \mathrm{NaF}$, the swelling decreases by $15 \%$. For the $\mathrm{Cl}^{-}$ions, there is around $30 \%$ decrease in swelling, while for $\mathrm{I}^{-}$, the decrease is a bit 
larger (about 35\%). According to the law of matching water affinity, chaotropic cations have a high binding affinity with chaotropic anions, while they have less binding affinity with kosmotropic anions. It was extracted from molecular dynamics simulations, ${ }^{14 \mathrm{~b}}$ that the ammonium group $\mathrm{N}^{+}\left(\mathrm{CH}_{3}\right)_{3}$ belongs to chaotropic ions. Therefore, it is likely to combine with the more chaotropic anion $\mathrm{I}^{-}$compared with $\mathrm{F}^{-}$and $\mathrm{Cl}^{-}$. The ionic interaction breaks the hydration and causes a slight collapse of brushes. Because of the stronger interaction, $\mathrm{I}^{-}$can induce a stronger collapse than the other two anions.

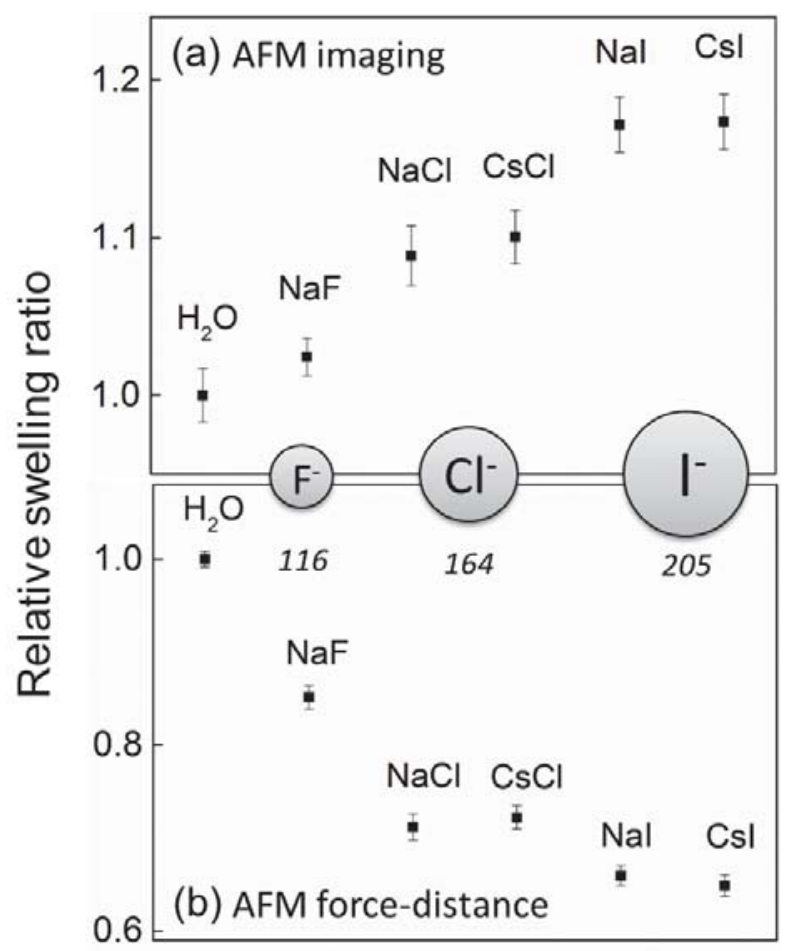

Counterions

Figure 8.1. The relative swelling ratio of PMPC brushes in $\mathrm{H}_{2} \mathrm{O}$, and $1 \mathrm{M}$ various salts solution measured by AFM force-distance (down) and imaging (up) using a normal load of $50 \mathrm{nN}$ over a scan size of $30 \mu \mathrm{m}$ at a scan rate of $0.5 \mathrm{~Hz}$. The radius unit is pm. The error bars denote the standard error of the mean with a $95 \%$ confidence interval.

For the imaging method (Figure 8.1 bottom), we observe a completely different trend. The PMPC brushes increase in swelling with adding salts. Moreover, with increase of the radius of anions, the brushes also swell more. While, for varying the cations from sodium to cesium, there is almost no change on the swelling ratio. For the smallest ions $\mathrm{F}^{-}$, there is only about $2 \%$ increase of the dimension. For $\mathrm{Cl}^{-}$, the brushes swell around $9-10 \%$ more. When $\mathrm{I}^{-}$is added in the solution, a $17 \%$ increase of the measured height is obtained. 


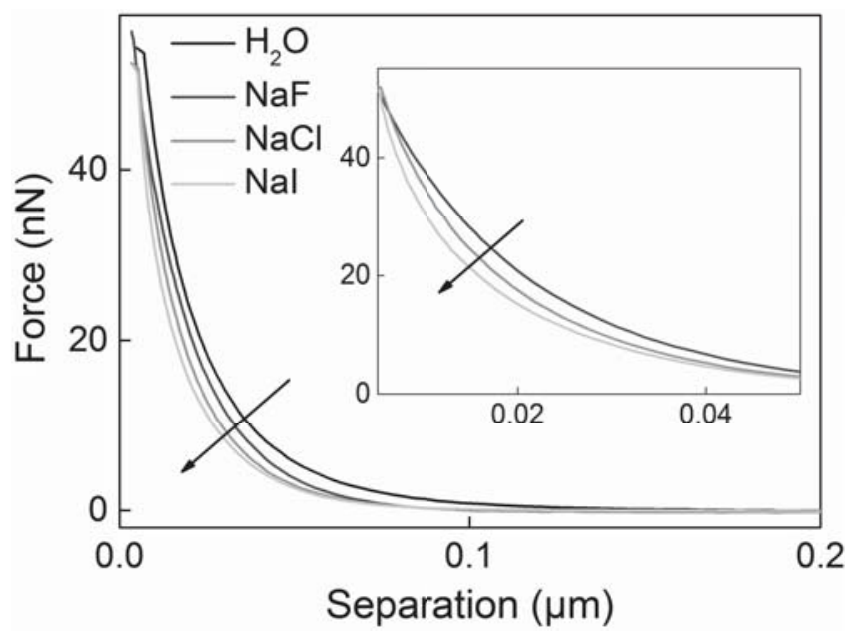

Figure 8.2. Typical approaching force versus separation curves at various salts aqueous solution, $\mathrm{H}_{2} \mathrm{O}, \mathrm{NaF}, \mathrm{NaCl}, \mathrm{NaI}$. The approach velocity of the PS colloid to the PMPC brushes is $2 \mu \mathrm{m} / \mathrm{s}$ with a load of $50 \pm 5 \mathrm{nN}$. The inset is the magnification of $\mathrm{NaF}, \mathrm{NaCl}$, $\mathrm{NaI}$ curves.

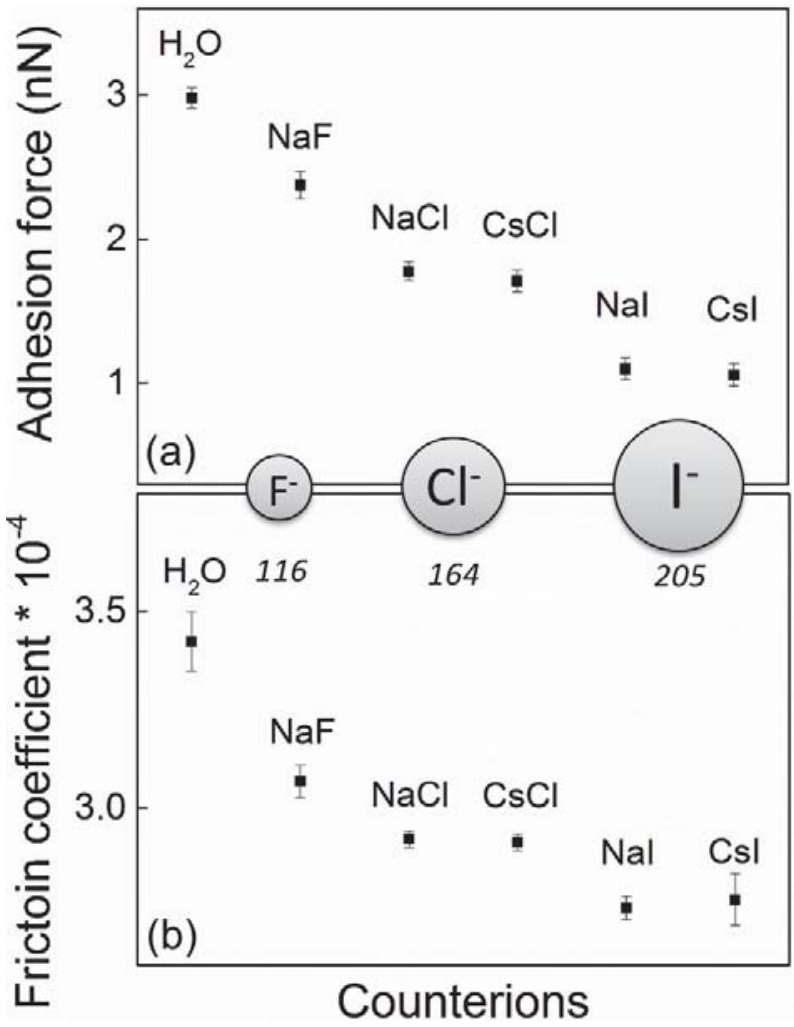

Figure 8.3. Adhesion force (a) and friction coefficient (b) between the PMPC brushes and a PS colloid as a function of ion radius (pm) measured by AFM over a scan size of $20 \mu \mathrm{m}$. The error bars denote the standard error of the mean with a $95 \%$ confidence interval. 
Why we obtain different results for these different extraction-techniques, becomes clear in Figure 8.2. Figure 8.2 shows force versus separation curves upon approach of the PS colloid towards the PMPC brush in pure water and in $1 \mathrm{M} \mathrm{NaF}, \mathrm{NaCl}$ and $\mathrm{NaI}$ solutions. In pure water the force already deviates from zero at large distances (180-200 nm). However, the water-filled brush is easily compressed by a small force. For a force of $2 \mathrm{nN}$, the separation goes down to $106 \mathrm{~nm}$. Upon adding salts, the force deviates from zero for much smaller separations $(60-80 \mathrm{~nm})$. Yet, the brushes in salt solution are less easily compressed. Thus, under compression by a sufficiently large force, the apparent height of the salted brushes can be higher than the height for brushes in water.

Figure 8.3. (a) gives the adhesion between a PS colloid and a PMPC brush measured upon retraction of the colloid from the brush in pure water and for various $1 \mathrm{M}$ salt solutions. Before retraction, a force of $50 \mathrm{nN}$ compressed the brush. We observe the highest adhesion $(3 \mathrm{nN})$ in water and the lowest force in a $1 \mathrm{M} \mathrm{NaI}$ solution $(1 \mathrm{nN})$. These results seem to be consistent with the change in compressibility of the brushes: the water-filled brush can be compressed easily, such that the contact area is large. The brushes in $\mathrm{NaI}$ are stiffer, such that the contact area is smaller than in water. However, the brushes have approximately the same degree of compression at $48 \mathrm{nN}$, as can be seen from the separation at $48 \mathrm{nN}$ for the different aqueous solutions in Figure 8.2. An alternative explanation could be that the change in the adhesion due to a change in electrostatic interactions between the cations adsorbed on the charged PS and the anions interacting with the ammonium groups in the PMPC. However, this would indicate a cationic size dependent effect, which we do not observe.

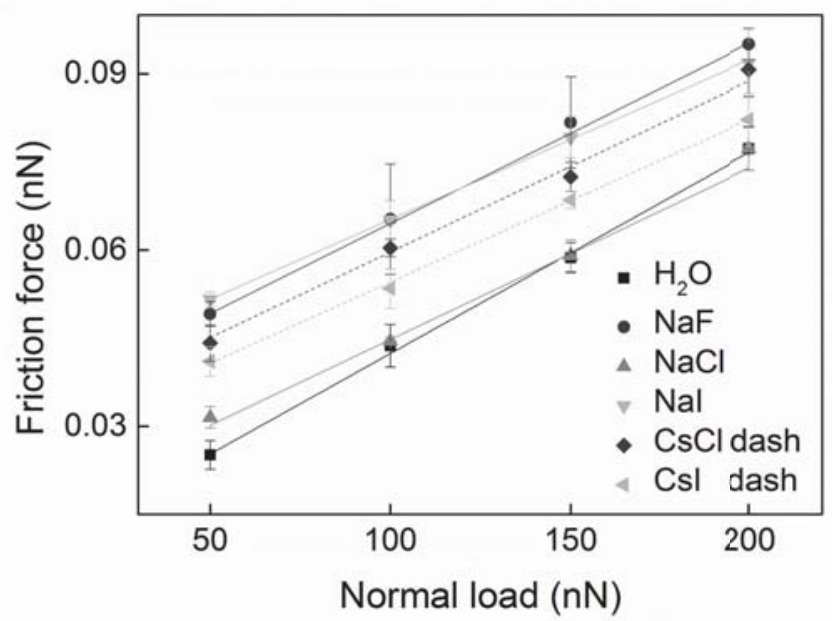

Figure 8.4. Friction force between the PMPC brushes and a PS colloid as a function of applied normal load measured at a relative velocity of $5 \mu \mathrm{m} / \mathrm{s}$, over a scan size of $10 \mu \mathrm{m}$. The error bars denote the standard error of the mean with a $95 \%$ confidence interval. 
Besides observing different effects on the swelling ratio, different research groups observed different effects of the addition of salts on the friction between PMPC brushes or for PMPC brushes. ${ }^{2,} 12 \mathrm{i}, 17$ While some observed that friction increases, ${ }^{17}$ others found that friction decreases. ${ }^{12 i}$ Figure 8.4 shows the friction measured upon sliding a PS colloid over a PMPC brush with a sliding velocity is $5 \mu \mathrm{m} / \mathrm{s}$ with a stroke length of $10 \mu \mathrm{m}$ for various normal loads between 50 and $200 \mathrm{nN}$. The results show that the effect of the addition of the different types of salt on the friction force depends on the normal load. Therefore, different effects can be observed for different groups.

In the bottom graph of Figure 8.3 we show the friction coefficient determined after a linear fit to the data in Figure 8.4. We observe that, by adding salts in the aqueous solution, there is a decrease in the friction coefficient, which is also following the order of specific ion effect. In NaF, there is around $10 \%$ decrease compared to the friction coefficient measured in $\mathrm{H}_{2} \mathrm{O}$. By changing the anion to $\mathrm{Cl}^{-}$and $\mathrm{I}^{-}$, the decrease in friction coefficient is around $15 \%$ and $20 \%$, respectively. Both adhesion and friction decrease by introducing ions in the solution. Moreover, both of them follow a decreasing order as $\mathrm{F}^{-}<\mathrm{Cl}^{-}<\mathrm{I}^{-}$. However, the differences in the friction coefficients are not as significant as the changes we observe for the adhesion. This seems to agree with the small difference in contact area for the brushes in different salt solutions under these normal loads, which indicates that friction is caused by visco-elastic brush deformation instead of electrostatic interactions with the colloid.

For polymeric systems in contact with a chemically different counter surface, friction and adhesion are often related. ${ }^{22}$ Though, there exist some counter-examples where friction and adhesion are caused by different mechanisms. ${ }^{23}$ Our results seem to indicate the adhesion and friction are caused by different mechanism, even though they show the same trend. Though, more research is needed to confirm this.

\subsection{Outlook}

To test if the changes in friction and adhesion are caused by visco-eleastic deformation in the brush or maybe electrostatic interaction with cations adsorbed onto the PS colloid, we would like to change the chemical composition of the colloid. Moreover, in the present experiments, we did not observe an effect of the cation on the brush swelling, friction and adhesion. The reason for this could be that the water-affinities of both $\mathrm{Na}^{+}$and $\mathrm{Cs}^{+}$did not match that of the phosphate group. Phosphates are known to be chaotropic. ${ }^{24}$ Therefore, we anticipate an effect of the more kosmotropic $\mathrm{Li}^{+}$ions. Especially since $\mathrm{Li}^{+}$ions are small and will be less hindered to reach the phosphate group close to the polymer backbone. 


\subsection{References}

[1] a) S. Lee and N. D. Spencer, SCIENCE-NEW YORK THEN WASHINGTON- 2008, 319, 575; b) M. Kobayashi, M. Terada and A. Takahara, Faraday discussions 2012, 156, 403-412; c) M. Kobayashi, Y. Terayama, N. Hosaka, M. Kaido, A. Suzuki, N. Yamada, N. Torikai, K. Ishihara and A. Takahara, Soft Matter 2007, 3, 740-746.

[2] M. Chen, W. H. Briscoe, S. P. Armes and J. Klein, science 2009, 323, 1698-1701.

[3] S. T. Milner, Science 1991, 251, 905-914.

[4] W. T. S. Huck, Materials Today 2008, 11, 24-32.

[5] I. Tokareva, S. Minko, J. H. Fendler and E. Hutter, Journal of the American Chemical Society 2004, 126 , $15950-15951$.

[6] a) O. Azzaroni, A. A. Brown and W. T. Huck, Angewandte Chemie 2006, 118, 1802-1806; b) Y. Ding, X. H. Xia and H. S. Zhai, Chemistry-a European Journal 2007, 13, 4197-4202; c) C. Leng, X. F. Han, Q. Shao, Y. H. Zhu, Y. T. Li, S. Y. Jiang and Z. Chen, Journal of Physical Chemistry C 2014, 118, 15840-15845; d) H. Matsuoka, Y. Yamakawa, A. Ghosh and Y. Saruwatari, Langmuir 2015, 31, 4827-4836; e) T. Okada and J. M. Patil, Langmuir 1998, 14, 6241-6248; f) H. J. Shen, M. Zhou, Q. Zhang, A. Keller and Y. Shen, Colloid and Polymer Science 2015, 293, 1685-1694.

[7] D. Y. Ko, M. Patel, B. K. Jung, J. H. Park and B. Jeong, Biomacromolecules 2015, 16, 3853-3862.

[8] a) J. Z. Du, Y. Q. Tang, A. L. Lewis and S. P. Armes, Journal of the American Chemical Society 2005, 127, 17982-17983; b) M. Ilcikova, J. Tkac and P. Kasak, Polymers 2015, 7, 2344-2370.

[9] Y. L. Yu, B. D. Kieviet, F. Liu, I. Siretanu, E. Kutnyanszky, G. J. Vancso and S. de Beer, Soft Matter 2015, 11, 8508-8516.

[10] Y. Higaki, M. Kobayashi, D. Murakami and A. Takahara, Polymer Journal 2016, 48, 325-331.

[11] a) K. Kitano, Y. Inoue, R. Matsuno, M. Takai and K. Ishihara, Colloids and Surfaces B-Biointerfaces 2009, 74, 350-357; b) G. Q. Liu, Z. L. Liu, N. Li, X. L. Wang, F. Zhou and W. M. Liu, Acs Applied Materials \& Interfaces 2014, 6, 20452-20463; c) T. Moro, Y. Takatori, M. Kyomoto, K. Ishihara, M. Hashimoto, H. Ito, T. Tanaka, H. Oshima, S. Tanaka and H. Kawaguchi, Journal of Orthopaedic Research 2014, 32, 369-376.

[12] a) M. M. A. E. Claessens, B. F. van Oort, F. A. M. Leermakers, F. A. Hoekstra and M. A. C. Stuart, Biophysical Journal 2004, 87, 3882-3893; b) Z. L. Cao and G. Z. Zhang, Physical Chemistry Chemical Physics 2015, 17, 27045-27051; c) C. C. Chang, R. Letteri, R. C. Hayward and T. Emrick, Macromolecules 2015, 48, 7843-7850; d) B. Lego, W. G. Skene and S. Giasson, Macromolecules 2010, 43, 4384-4393; e) J. Mahon and S. Zhu, Colloid and Polymer Science 2008, 286, 1443-1454; f) L. Ni, J. Meng, G. M. Geise, Y. Zhang and J. Zhou, Journal of Membrane Science 2015, 491, 73-81; g) K. Ogawa, Advances in colloid and interface science 2015, 226, 115-121; h) J. T. Yang, H. Chen, S. W. Xiao, M. X. Shen, F. Chen, P. Fan, M. Q. Zhong and J. Zheng, Langmuir 2015, 31, 9125-9133; i) Z. Y. Zhang, M. Moxey, A. Alswieleh, A. J. Morse, A. L. Lewis, M. Geoghegan and G. J. Leggett, Langmuir 2016, 32, 5048-5057; j) J. de Grooth, M. Dong, W. M. de Vos and K. Nijmeijer, Langmuir 2014, 30, 5152-5161.

[13] F. Hofmeister, Arch. Exp. Pathol. Pharmakol.(Leipzig) 1888, 24, 247-260.

[14] a) S. Z. Moghaddam and E. Thormann, Journal of Colloid and Interface Science 2016, 465, 67-75; b) A. Salis and B. W. Ninham, Chemical Society Reviews 2014, 43, 7358-7377; c) N. Vlachy, B. Jagoda-Cwiklik, R. Vacha, D. Touraud, P. Jungwirth and W. Kunz, Advances in Colloid and Interface Science 2009, 146, 42-47; d) Y. Zhang, S. Furyk, L. B. Sagle, Y. Cho, D. E. Bergbreiter and P. S. Cremer, The Journal of Physical Chemistry C 2007, 111, 8916-8924.

[15] X. Chen, T. Yang, S. Kataoka and P. S. Cremer, Journal of the American Chemical Society 2007, 129, $12272-12279$.

[16] B. A. Humphreys, J. D. Willott, T. J. Murdoch, G. B. Webber and E. J. Wanless, Physical Chemistry Chemical Physics 2016, 18, 6037-6046.

[17] M. Kobayashi, Y. Terayama, M. Kikuchi and A. Takahara, Soft Matter 2013, 9, 5138-5148.

[18] S. Morozova, G. Hu, T. Emrick and M. Muthukumar, Acs Macro Letters 2016, 5, 118-122.

[19] Q. Shao, L. Mi, X. Han, T. Bai, S. J. Liu, Y. T. Li and S. Y. Jiang, Journal of Physical Chemistry B 2014, $118,6956-6962$.

[20] a) J. B. Schlenoff, Langmuir 2014, 30, 9625-9636; b) T. Wang, R. Kou, H. L. Liu, L. D. Liu, G. Z. Zhang and G. M. Liu, Langmuir 2016, 32, 2698-2707.

[21] Y. Matsuda, M. Kobayashi, M. Annaka, K. Ishihara and A. Takahara, Langmuir 2008, 24, 8772-8778. [22] a) X. Banquy, E. Charrault and S. Giasson, Journal of Physical Chemistry B 2010, 114, 9721-9728; b) M. Raftari, Z. Y. Zhang, S. R. Carter, G. J. Leggett and M. Geoghegan, Soft Matter 2014, 10, 2759-2766; c) A. Li, S. N. Ramakrishna, E. S. Kooij, R. M. Espinosa-Marzal and N. D. Spencer, Soft Matter 2012, 8, 9092-9100; d) J. Song and G. J. Vancso, Langmuir 2011, 27, 6822-6829; e) M. K. Vyas, K. Schneider, B. Nandan and M. Stamm, Soft Matter 2008, 4, 1024-1032. 
[23] a) J. Huang, B. Cusick, J. Pietrasik, L. Wang, T. Kowalewski, Q. Lin and K. Matyjaszewski, Langmuir 2007, 23, 241-249; b) N. Nordgren and M. W. Rutland, Nano Letters 2009, 9, 2984-2990.

[24] K. D. Collins, Biophysical journal 1997, 72, 65. 


\section{Summary}

Controlling friction and adhesion is relevant in nature and in our daily life. Such control can be achieved using stimulus responsive end-anchored polymers forming a brush. These brushes can adapt their physicochemical properties upon changing the surrounding environments, such as temperature, light, $\mathrm{pH}$, electrical and magnetic fields. In this Thesis, we primarily use atomic force microscopy (AFM) to study the relation between the degree of solvation of the macromolecules in the brush and the friction and adhesion. In general, it is accepted that collapsed brushes promote high friction/adhesion and swollen brushes exhibit low friction/adhesion. However, our results show that brush swelling and adhesion/friction is not necessarily related. We show that the relation between swelling of a brush and its tribomechanical properties is rather complex, and that it can depend on the specific interactions in the contact for the different studied systems: for chemically identical/different brush-brush contact or for brush-solid counter surface contacts. The switchable adhesion and friction we observed using stimulus-responsive polymer brushes have potential applications in pick up-release system, robots and in biomedical systems.

Chapter 1 gives a short introduction to the topics related to the Thesis and the main research in the following chapters.

In the first paragraphs of Chapter 2, we give an introduction to the generic concepts and definitions employed in this Thesis, including the polymer brush, AFM, adhesion, friction and stimulus-responsive polymers. In the second part of the chapter, we summarize literature studies on switchable friction and adhesion by stimulus-responsive polymer films, gels and brushes in contact with chemically identical or different counter surfaces. In the last paragraphs we discuss and give some examples on the relationship between friction and adhesion/adhesion hysteresis.

In Chapter 3, we describe the tribo-mechanical properties of thermally responsive $\operatorname{poly}(\mathrm{N}$ isopropyl acrylamide) (PNIPAM) brushes. By varying the temperature below, around and above the lower critical solution temperature (LCST), switchable friction and adhesion between the brush and a gold colloid are obtained. In particular, an enhanced dissipation and friction are observed near the LCST of PNIPAM, which we attribute to the stretching of partly collapsed polymer brush chains that adhere to the gold colloid. The results have important implications for the development of polymer-based switchable tribo-mechanical systems (such as smart tweezers or artificial muscles).

In Chapter 4, we explore the enhanced friction and dissipation of PNIPAM brushes due to the co-non-solvency effect: PNIPAM brushes are swollen in pure solvents, and precipitate in a certain solvent-cosolvent mixture. Both in water and in ethanol, the brushes are swollen and low friction is obtained. In 30\% volume fraction of ethanol-water mixture, the brush collapses the most, but the friction is not the highest. Surprisingly, in 10-90 vol.\% ethanol- 
water, a maximum in friction is observed, which is again related to the stretching of partially collapsed PNIPAM brushes. The highest friction is about two orders of magnitude larger than the lowest friction in ethanol.

Besides friction, the adhesion forces between PNIPAM brushes and various colloids, like silica, gold, polystyrene (PS), poly(methyl methacrylate) (PMMA), are also characterized by employing the co-non-solvency effect. Switchable adhesion can be obtained for all these tested colloids, but the magnitude of the adhesion-change varies. The application of co-nonsolvency of PNIPAM brushes to pick up, move and release nanoparticles is studied in Chapter 5. In a water-ethanol mixture of $30 \%$ ethanol, the brush-particle adhesion is the highest such that particles can be picked up. In pure ethanol, the brushes swell and the adhesion is strongly reduced such that particle-release can be triggered. We employ the change in adherence to transfer silicon nanoparticles from one liquid into another. The advantage of the system is that the formation of aggregates is strongly reduced, which is an important problem in traditional methods of nanoparticle transfer. Here, the highest friction and adhesion are not found at the same composition of ethanol and water, which shows that they are not necessarily related.

In Chapter 6, cosolvency induced switchable adhesion between PMMA brushes is described: PMMA cannot be dissolved in water and alcohols, but can be swollen in a certain mixture of them. In poor solvents for PMMA, like water, ethanol and isopropanol, there is a high contact adhesion between PMMA brushes. However, in a mixture of water and one of the alcohols $(\mathrm{v} / \mathrm{v}=4 / 5)$ the brushes swell and a low adhesion between the brushes is observed. Compared with ethanol-water system, which exhibits numerous pulling events due to chains interdigitation, the isopropanol-water system is the better choice for capture-and-release system.

In Chapter 7, we describe a new and facile method to make zwitterionic poly(2methacryloyloxyethyl phosphorylcholine) (PMPC) brushes using a poly(glycidyl methacrylate) (PGMA)-based macro-initiator. Our PGMA-PMPC brushes are shown to be very stable in pure water and salt solutions. Only $1 \%$ of the polymers detach after immersion for 4 weeks in saline solution or in artificial seawater. In contrast, PMPC brushes prepared using the traditional silane-based method show a strong ion-specific degrafting, which can be explained by the difference in polymer stretching in the different aqueous media. The stable PMPC brushes have potential application in biomedical engineering.

In Chapter 8, we show there are specific ion effects on the hydration of zwitterionic PMPC brushes and they can be employed to control friction and adhesion. Via different methods to extract the swelling from our AFM results, we find an opposite effect of the addition of salts to the hydration behavior of PMPC brushes: Force-distance results show a decrease in swelling by adding salts. While AFM imaging results show the opposite trend, as the 
brushes in salt solution are less easily compressed under the applied load. The friction coefficient decreases by adding salts, and decreases more with the increase of the anion radius. In contrast, the adhesion between the PS colloid and the PMPC brush does not relate to brush swelling and instead may arise from electrostatic interactions. We conclude this chapter with an outlook to future experiments that can clarify our observations. 



\section{Samenvatting}

Het beheersen van wrijving en adhesie is relevant in de natuur, maar ook in ons dagelijks leven. Dit kan bereikt worden met behulp van polymeren die aan één kant vast zitten aan een oppervlak, zodat ze een zogenaamde polymeer borstel vormen, en die reageren op een opgelegde stimulus. Zulke borstels kunnen hun fysisch-chemische eigenschappen aanpassen wanneer de omgeving, zoals temperatuur, lichtintensiteit, $\mathrm{pH}$, etc., verandert. In dit proefschrift beschrijven we hoofdzakelijk atoomkrachtsmicroscopie metingen waarin we de relatie tussen het opzwellen van polymeerborstels en de bijbehorende tribologische eigenschappen bestuderen. Over het algemeen wordt er gesteld dat borstels in slechte oplosmiddelen voor een hoge adhesie en wrijving zorgen, terwijl borstels in een goed oplosmiddel lage wrijving en adhesie laten zien. Onze resultaten laten echter zien dat het opzwellen van de borstels en wrijving/adhesie niet noodzakelijk direct gerelateerd zijn en dat de exacte relatie afhangt van specifieke interacties in het contact gebied. De beheersing van de wrijving en adhesie die we bereikt hebben in dit proefschrift zal uiteindelijk gebruikt kunnen worden in de robotica of in biomedische systemen.

In Hoofdstuk 1 beschrijven we de inhoud van dit proefschrift en wat er specifiek te vinden is in de verschillende hoofdstukken.

In de eerste paragrafen van Hoofdstuk 2 geven we de lezer een algemene introductie tot de concepten en technieken die in het onderzoek beschreven in dit proefschrift gebruikt worden. In het tweede gedeelte van het hoofdstuk beschrijven we de huidige status van het onderzoek door anderen met betrekking tot het beheersen van adhesie en wrijving waarin ze gebruik maken van polymeren die reageren op een externe stimulus.

In Hoofdstuk 3 beschrijven we ons onderzoek aan poly( $N$-isopropyl acrylamide) (PNIPAM) borstels die we van een silicium oppervlak gegroeid hebben. Dit type polymeer heeft een zogenaamde 'lagere kritische oplostemperatuur' (LCST) in water: boven deze temperatuur is water een slecht oplosmiddel voor PNIPAM en onder deze temperatuur is het een goed

oplosmiddel. In tegenstelling tot de algemene verwachtingen vonden we dat de wrijving tussen PNIPAM en een gouden colloïde maximaal is rond de LCST in plaats van boven de LCST. Deze wrijvingstoename lijkt te komen door het uitstrekken van de al gedeeltelijk dicht bij het oppervlak liggende polymeren wanneer de colloïde weg beweegt van het initiële contactgebied.

In Hoofdstuk 4 presenteren we ons onderzoek naar de toename in wrijving voor PNIPAM borstels door het zogenaamde 'co-non-oplosbaarheid' effect. In de pure, goede oplosmiddelen ethanol en water, is de PNIPAM borstel opgezwollen, terwijl de borstel neerslaat in een mengsel van de twee oplosmiddelen. We observeerden dat de borstel minimaal opgezwollen was voor een 30-70 vol.\% ethanol-water mengsel, maar dat de 
wrijving het hoogst was voor een 10-90 vol.\% ethanol-water mengsel. Ook hier lijkt dit veroorzaakt te worden door het uitstrekken van al gedeeltelijk neergeslagen polymeren.

In Hoofdstuk 5 laten we zien dat dit co-non-oplosbaarheid effect gebruikt kan worden om nanodeeltjes op te pakken in een 30-70 vol.\% ethanol-water mengsel en dat deze deeltjes daarna weer losgelaten kunnen worden in pure oplosmiddelen. Het gebruik van een polymeerborstel hiervoor heeft als voordeel dat er voorkomen wordt dat er grote clusters gevormd worden.

In Hoofdstuk 6 presenteren we een alternatieve manier voor het beheersen van de adhesie voor het oppakken en loslaten van deeltjes. We laten zien dat de adhesie tussen poly(methyl methacrylaat) (PMMA) borstels veranderd kan worden door gebruik te maken van het zogenaamde 'co-oplosbaarheid' effect. In pure oplosmiddelen (ethanol/isopropanol of water) zijn de PMMA borstels neergeslagen en is de adhesie hoog, terwijl in een 80-20 vol.\% de borstels gedeeltelijk opzwellen en de adhesie lager is.

In Hoofdstuk 7 beschrijven we een simpele manier om ervoor te zorgen dat hydrofiele zwitterionische poly(2-methacryloyloxyethyl phosphorylcholine) (PMPC) polymeren in borstels niet loskomen van het oppervlak door hydrolyse van de verbindingen met het oppervlak. We bereiken dit door de borstels van poly(glycidyl methacrylate) (PGMA) macro-initiators te groeien. Voor deze PGMA-PMPC borstels meten we dat maar 1\% van de polymeren losgekomen is van het oppervlak na 4 weken ondergedompeld te zijn geweest in saline oplossing of zeewater.

In Hoofdstuk 8 presenteren we de eerste resultaten van ons onderzoek aan specifieke ionen effecten op het opzwellen PMPC borstels en op de wrijving / adhesie tussen deze polymeerborstels en een polystyreen colloïde deeltje. We observeren dat het gemeten effect van verschillende zouten op borstel-zwellen afhangt van de gebuikte meettechniek, omdat zowel de absorptie van het oplosmiddel als de samendrukbaarheid van de borstel afhangt van het type anion. Naarmate de hydrodynamische radius van het anion toeneemt, neemt het opzwellen en de samendrukbaarheid af. Hierdoor lijkt het opzwellen toe te nemen als de hoogte gemeten wordt onder een positieve normaalkracht. Voor de minst opgezwollen borstels vinden we dat de wrijving en adhesie het laagst is. Daarnaast geven we een vooruitblik naar toekomstige experimenten die we gepland hebben. 


\section{Acknowledgements}

Finally I am here to write the acknowledgements after 4 years' $\mathrm{PhD}$ life in the Netherlands. In Chinese, working as a $\mathrm{PhD}$ has the same pronunciation as gambling. Try to think about it, in some cases, it really makes sense. :p I still remember it was a rainy day when I first arrived in this country. Then I started my PhD-adventure. At the beginning, I was totally excited and full of expectation that I could win. During the process, like the Dutch weather, sometimes it is a really sunny day. However, most of the days are cloudy and rainy. Sometimes I doubt myself, if it was wrong to start this "gambling", if it would be another situation by working in China, if it would be better I made another choice. But there is no if in the real life. After exploring, finally I stand almost there. Even lots of suffering on the way, I am still greatly touched by many people, whoever help me in these years. With all your helps, the $\mathrm{PhD}$ pursuing becomes more like an experience rather than a gambling.

First, I'd like to thank my promoter: Julius Vancso. Thanks for giving me this opportunity to pursue my $\mathrm{PhD}$ study in MTP group. Thanks for all your comments and efforts on my presentation and manuscripts. Your broad knowledge and precise attitude towards science really set a good example for all of us. I can learn a lot from you every time after the discussion. Especially on the Dutch Polymer Day, you always walk by and read my poster, and tells me it is really nice. Thanks for your encouragement, and that really makes me happy to keep working on the research. Three years ago, we had the interview on skype, and you introduced a young lady to be my daily supervisor. Yes, Sissi de Beer.

Sissi, I really do not know how to express my great appreciation to you. As my daily supervisor, you really teach me a lot to make me from an AFM-zero person to an AFMalways using person. :p I still remember, you started to teach me how to use AFM on $2^{\text {nd }}$ of January, 2014. There I start my first AFM experiments. You always try to teach me carefully and patiently, even I bother you many times on the same problem. Your big smile always encourages me a lot when I feel down and fail in the experiments. Though the weather here is always cloudy and rainy, your smile is like warm and bright sunshine. I really appreciate that you always stand on my side when there is criticism on me. With your protection, I really like to work hard and get more outcomes with you together to make us "famous in the science". :p Also I'd like to thanks for your carefully revision on my paper and thesis. Sometimes I complain you are too obsessive on writing a sentence over and over again, but I know that is good to improve my writing skill by this practicing. Your active attitude to science and lift really inspired me, and I should learn from you always to face all the troubles and difficulties with a big smile. :)

Next, I'd like to thank my paranymphs: Bart D. Kieviet (matlab expert) and Marco Cirelli (Ellipsometry expert). Bart, thanks for your help to extract data from the AFM results by matlab, otherwise I need to figure it out from thousands of files manually. Thanks for your discussion on my project and a lot of nice suggestions. Say hello to your little son, he is so 
cute. Marco, I really like your ahla-ahla greetings and humor, which really makes me relaxed after the tough and hard work. :) Also thanks for your efforts on ellipsometry analysis. Those similar result curves could drive me crazy without you. Moreover, thanks for your guys as my paranymphs, and it really means a lot to me, not only on science, but also on friendship.

Dear Clemens, thanks for your support on arranging all the financial stuff for me. Without that I could not stay here for the last three months. Also thanks for ordering the expensive cantilever and monomers for my experiments. Dear Geneviève, thanks you so much for helping me with the cover and scheme in the Thesis. Marion, thanks for helping me to get the cinema voucher by calling the gift company again and again in last Christmas. Finally, I get it and really enjoy the films there. :p

Dear Kaihuan, we met each other on Dutch Polymer Day in 2013, and we shared the same room by the mistake arrangement of the hotel. But after one year, we become colleague and friend in the same group. As the saying says, the world is too small. :p Thanks for your sharing with interesting topics of science and all the discussions on my project. Also I really miss the BBQ you made at your home with your girlfriend Sijia. Thanks for all the enjoyable time together after work.

Dear Xiaoping, Quan, Kai, Matija, Roman, Serhii, I really miss the hanging out together and happiness time in Delft. Thanks for your guys' accompanying in the cold winter there. Wish you guys success in your $\mathrm{PhD}$ and work there.

I also want to express my appreciation to the past and present MTP members during my $\mathrm{PhD}$ here. Thanks for all your help, and your company: Aysegul, Xueling, Hairong, Mark, Joost, Michel, Hubert, Lucie, Maria, Yan, Shanqiu, Elio, Andreas, Lehani, Sida, Chongnan, of course my students Erik, Mathies and Thomas. :) Also some people from other groups are acknowledged for your kind help: Simone, Igor, Fei, Aram, Shirish, Shazia, Xiumei, Jie, Wei, Liang, Liulin, Shuqin, He, Huiyu, Minmin, Lijie, Shuijing, Rong, Zhengchao, Haichuan, Zheng, Yibo. Thank you so much for the great time we have together.

Last and the most importantly, I'd like to thank all my families' support during my whole $\mathrm{PhD}$ period in the Netherlands. 老爸, 老妈, 谢谢你们一直以来没有给我强加任何要求 和负担，总是让我选择适合自己的路。老弟，弟妹，谢谢你们在家对父母的照顾， 还要谢谢我可爱的小侄女, 每次视频都萌萌哒, 能让我很开心地笑, 忘掉科研上的 烦恼。Also a lot of thanks to my cousins: Kun and Hongjin. Your encouragement and supporting give me enough motivation and courage to go through this adventure. Always love you, my families.

Yunlong Yu 于云龙

Enschede, the Netherlands

December, 2016 


\section{Curriculum Vitae}

Yunlong Yu
Phone: +31-685799808 E-mail: yuyunlong666@gmail.com
EDUCATION
1/2014-1/2017 University of Twente
PhD in Materials Science and Technology of Polymers (MTP) group
9/2009-7/2012 Sichuan University
M. Sc. in polymer science and engineering, academic ranking, Top 10\% out of 150
students
9/2004-7/2008 North University of China
B. Sc. in polymer material and engineering, academic ranking, Top 10\% out of 100
students

\section{RESEARCH EXPERIENCE}

- Switchable friction and adhesion by stimulus-responsive polymer brushes

- Preparation of magnetic micelles using ionic liquid surfactants

- Synthesis and characterization of novel cholesterol containing polymers based on double bond

- Preparation and characterization of micro-sized PS microspheres

- The study of PP filled with nano- $\mathrm{CaCO}_{3}$

\section{PUBLICATIONS}

- 9. Yunlong Yu, Marco Cirelli, Bernard D. Kieviet, E. Stefan Kooij, G. Julius Vancso, Sissi de Beer, Tunable Friction by Employment of Co-non-solvency of PNIPAM Brushes, Polymer, 2016, 102, 372.

- 8. Yunlong Yu, Bernard D. Kieviet, Edit Kutnyanszky, G. Julius Vancso, Sissi de Beer, Cosolvency-Induced switching of the adhesion between PMMA brushes, $\boldsymbol{A C S}$ Macro Lett., 2015, 4, 75.

- 7. Yunlong Yu, Bernard D. Kieviet, Fei Liu, Igor Siretanu, Edit Kutnyánszky, G. Julius Vancso, Sissi de Beer, Stretching of collapsed polymers causes an enhanced dissipative response of PNIPAM brushes near their LCST, Soft Matter, 2015, 11, 8508.

- 6. Yunlong Yu, Junwei Bai, Junhua Zhang, Synthesis of side-chain cholesterol derivatives based on double bond, Journal of Molecular structure, 2012, 1019, 1.

- 5. Yunlong Yu, Junhua Zhang, Synthesis and characterization of novel cholesterol derivatives with or without spacer, Journal of molecular structure, 2012, 1012, 12.

- 4. Yunlong Yu, Haiyan Du, Junhua Zhang, Self-assembly of novel cholesterol derivative based on hydrogen bond, Journal of Molecular Structure, 2011, 1005, 107.

- 3. Haiyan Du, Yunlong Yu, Junhua Zhang, Microwave induced shape memory effect of cross-linked PVA, Macromolecular Chemistry and Physics, 2011, 212, 1460 .

- 2. Yunlong Yu, Junhua Zhang, Synthesis and characterization of cholesteric derivatives based on the side double bond, Polymer Material Science and Engineering, 2011. (In Chinese)

- 1. Yunlong Yu, Junhua Zhang, Synthesis and characterization of novel cholesteric derivatives and corresponding liquid crystalline polymers. Chinese Journal of Synthetic Chemistry, 2011. (In Chinese) 
SOCIAL ACTIVITIES

10/2014-9/2015 Secretary of Association of Chinese Students and Scholars in the Netherlands-Enschede

\section{9/2010-6/2011 State Key Lab of Polymer Material and Engineering} Chengdu

- Position: Instrument secretary

- Responsibility and Achievement:

- Responsibility for Thermal Gravimetric Analysis (TGA), assurance of test in good condition.

- Responsibility for Fourier Transform Infrared Spectroscopy (FTIR), operate the instrument and offer results to customers.

- Resolution of temporary conflict of customers, improvement of interpersonal skill.

- Improvement of the practical skill, and strengthen the sense of responsibility.

\section{4/2008-4/2007 North University of China}

Taiyuan

- Position: Study Minister of the University

- Responsibility and Achievement:

- Lead the entire students in university to study in a scientific method and organize all kinds of activities and contest to help improving the ability of the students.

- Improvement of my leader skill, and strengthen the communicative competence.

\section{HONOR AND AWARDS}

- 2016 The $3^{\text {rd }}$ poster prize at the MESA+ Meeting, 2016.

- 2016 The Poster Prize at Dutch Polymer Day in the Netherlands.

- 2015 The Best Research Proposal on the Soft Matter Day at the University of Twente.

- 2012 First-class Scholarship of Shenzhen Senior Technology Material Co., LTD.

- 2011 Second-Class Scholarship for Excellent Master of Sichuan University.

- 2010 Excellent master of Sichuan University

- 2007 Prominent student in summer practice

- 2005 The Second prize of English Contest of University Students in Shanxi Province

- 2005 Excellent student leader 


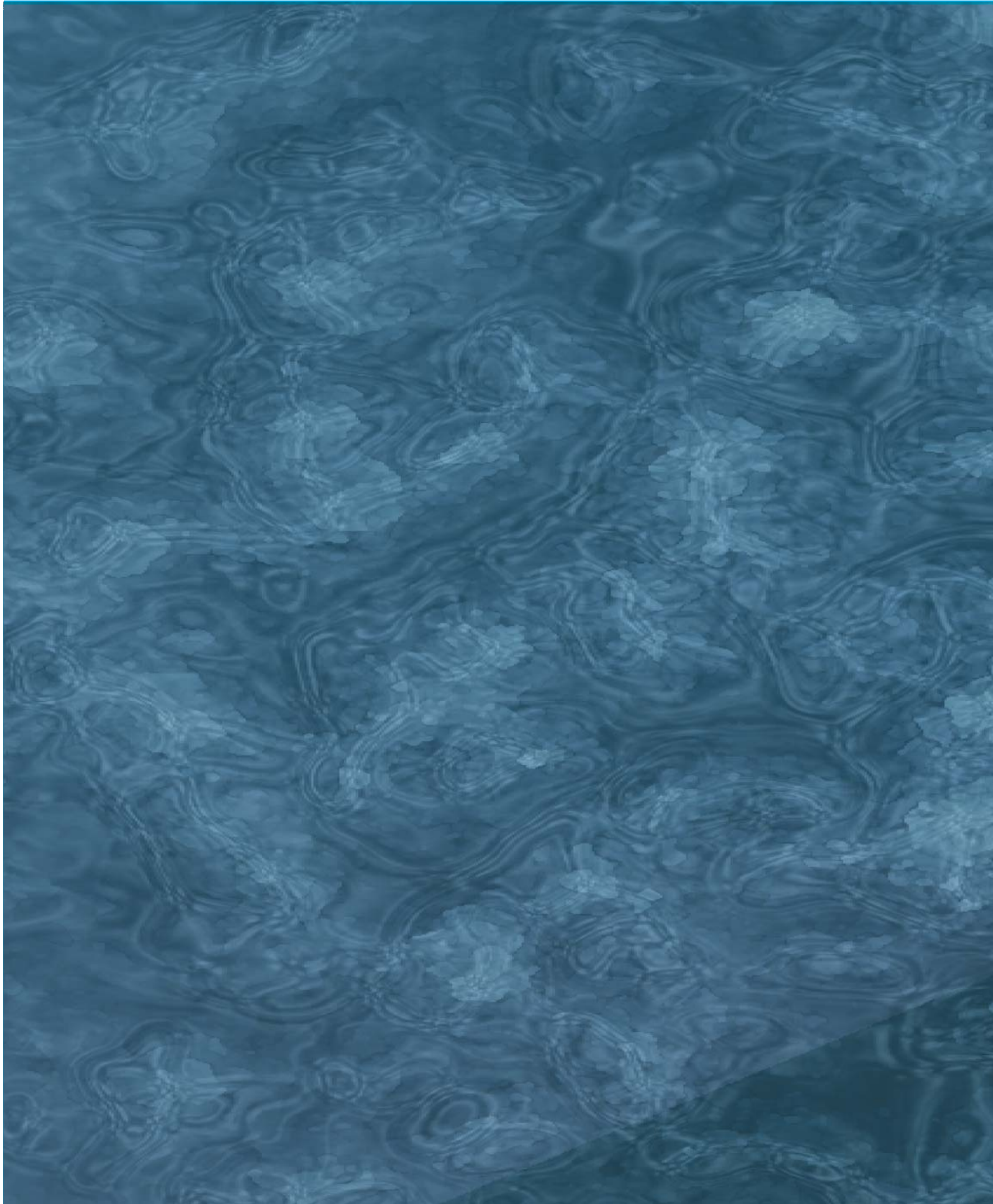

ISBN 978-90-365-4280-7

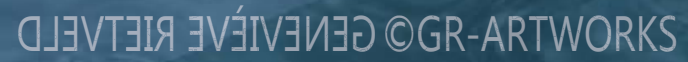

